\title{
DESIGN AND FABRICATION TECHNIQUES OF DEVICES FOR EMBEDDED POWER ACTIVE CONTACT LENS
}

\author{
A Thesis \\ presented to \\ the Electrical Engineering Department Faculty of \\ California Polytechnic State University, San Luis Obispo
}

\author{
In Partial Fulfillment \\ of the Requirements for the Degree \\ Master of Science in Electrical Engineering \\ by \\ Errol Leon
}

June 2015 
(C)2015

Errol Leon

ALL RIGHTS RESERVED 


\section{COMMITTEE MEMBERSHIP}

TITLE: Design and Fabrication Techniques of Devices for Embedded Power Active Contact Lens

AUTHOR: $\quad$ Errol Leon

DATE SUBMITTED: June 2015

COMMITTEE CHAIR: Tina Smilkstein, Ph.D.

Assistant Professor of Electrical Engineering

COMMITTEE MEMBER: Richard Savage, Ph.D.

Professor of Biomedical Engineering

COMMITTEE MEMBER: John Dunning, Ph.D.

Research Scholar of Mechanical Engineering 


\begin{abstract}
Design and Fabrication Techniques of Devices for Embedded Power Active Contact Lens

Errol Leon
\end{abstract}

This thesis designed and fabricated various devices that were interfaced to an IC for an active contact lens that notifies the user of an event by detection of an external wireless signal. The contact lens consisted of an embedded antenna providing communication with a $2.4 \mathrm{GHz}$ system, as well as inductive charging at an operating frequency of $13.56 \mathrm{MHz}$. The lens utilized a CBC005 $5 \mu \mathrm{Ah}$ thin film battery by Cymbet and a manufactured graphene super capacitor as a power source. The custom integrated circuit (IC) was designed using the On Semiconductor CMOS C5 $0.6 \mu \mathrm{m}$ process to manage the battery and drive the display. A transparent, flexible, single cell display was developed utilizing electrochromic ink to indicate to the user of an event. Assembly of the components, encapsulation, and molding were implemented to create the final product. The material properties of the chosen substrate were analyzed for their clearness, flexibility, and biocompatibility to determine its suitability as a contact lens material. Finally, the two different fabrication techniques (microfabrication and screen printing) that were employed to make the devices are compared to determine the favorable process for each part of the system.

Keywords: contact lens, integrated circuit, antenna, printing, microfabrication, super-capacitor, inductive-charging electrochromic, wire-bonding, encapsulation, biocompatibility. 


\section{ACKNOWLEDGMENTS}

I would like to take this opportunity thank Dr. Tina Smilkstein, Dr. Richard

Savage, and Dr. Malcom Keif for lending me their knowledge, guidance, and use of their facilities to manufacture a prototype. Also Grace Lao for her help in synthesis of polymers and materials for the power source. Finally, CP Connect and Hannah Forbes for contributing funds toward this research. 


\section{TABLE OF CONTENTS}

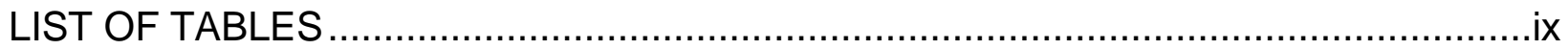

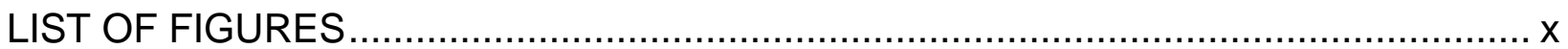

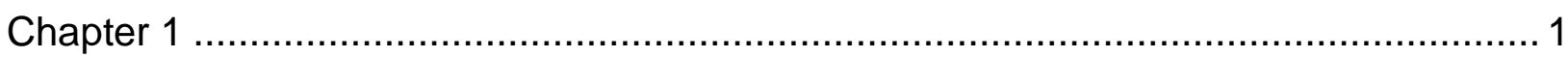

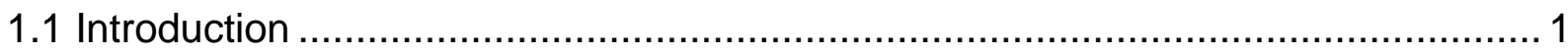

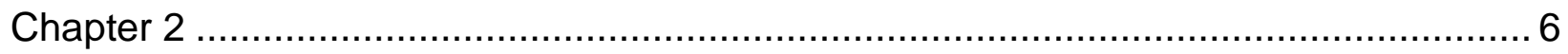

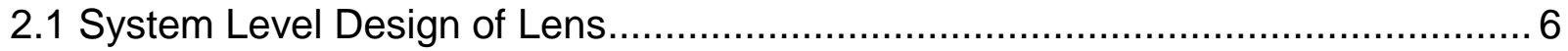

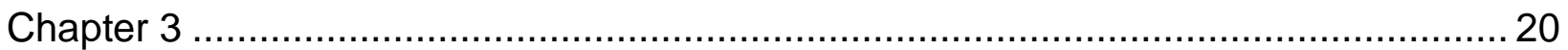

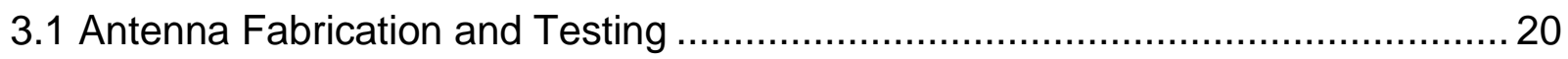

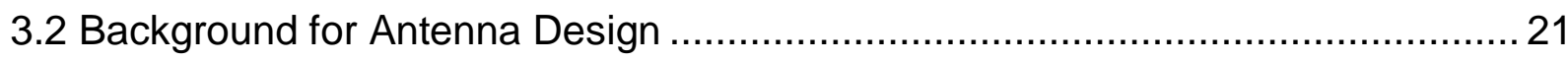

3.3 Printing Fabrication Process ..................................................................... 32

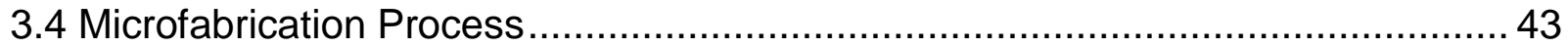

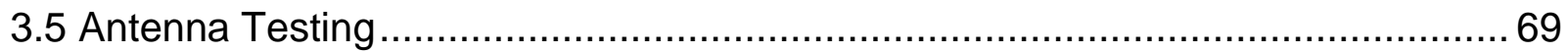

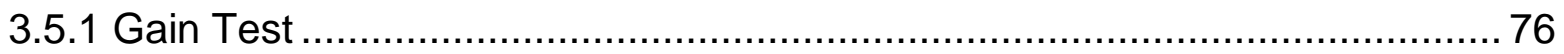

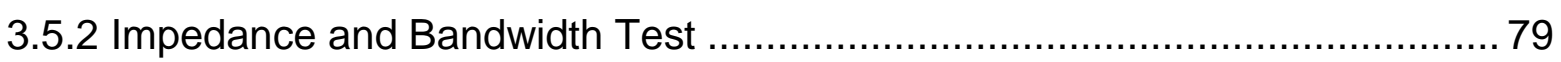

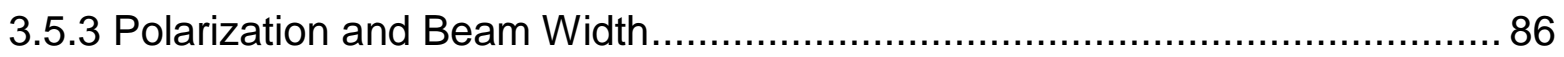

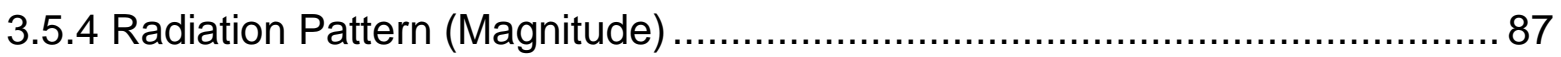

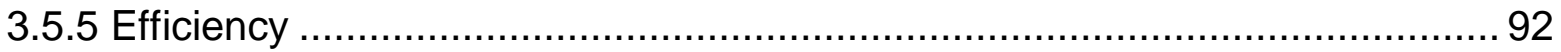

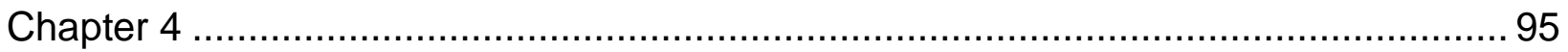

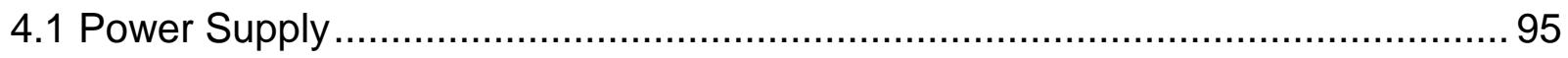

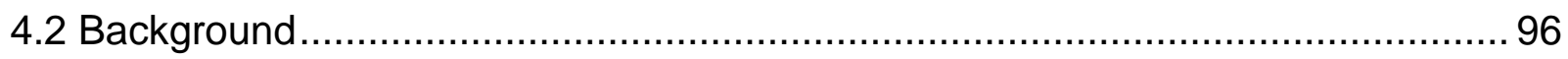

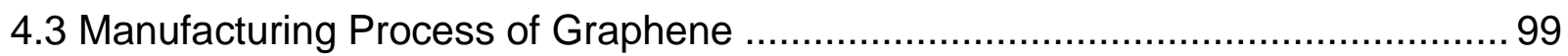

4.3.1 Synthesis using Chemical Vapor Deposition ............................................. 99

4.3.2 Chemical Synthesis of Graphene Film................................................. 100

4.4 Synthesis of Graphene Base Electrode …................................................ 102

4.5 Result of Graphene Based Electrode Super Capacitor ...................................... 102

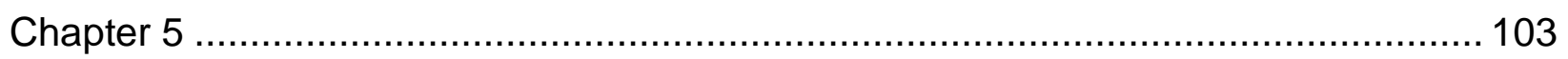

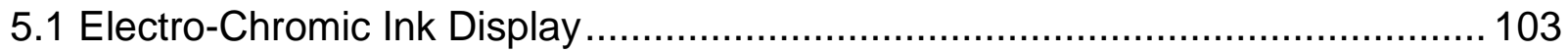

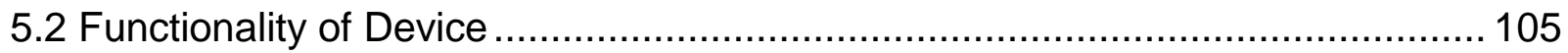

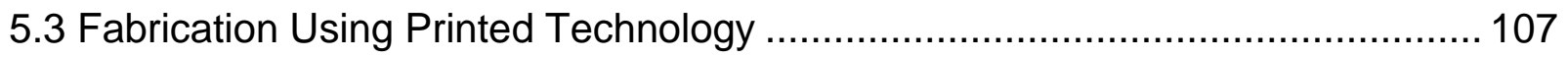

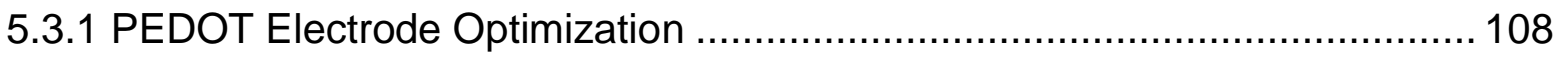

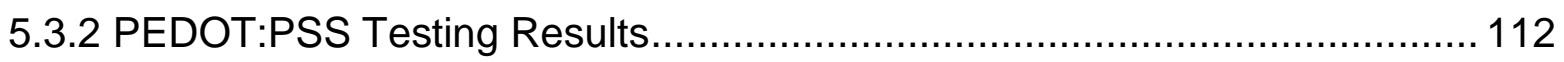

5.3.3 Electrolyte Manufacturing and Optimization........................................... 114 


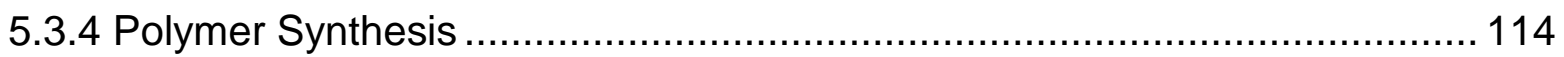

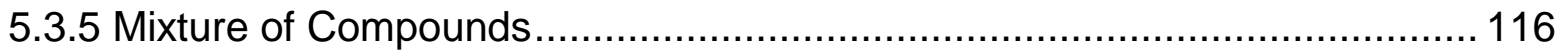

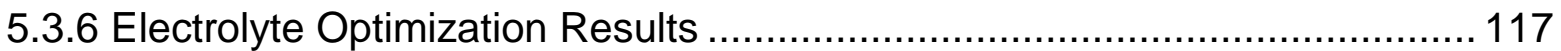

5.3.7 Printing Technology Process for Display ................................................ 118

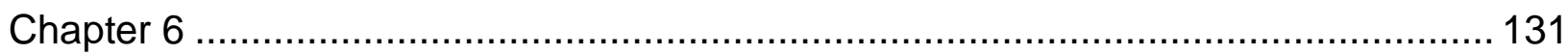

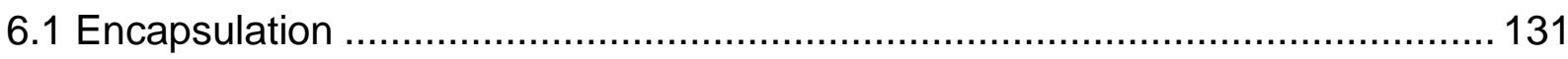

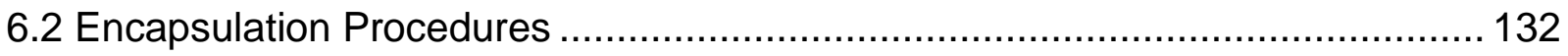

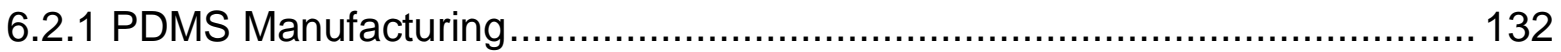

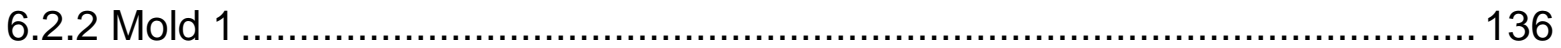

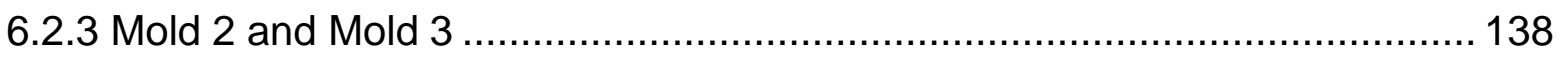

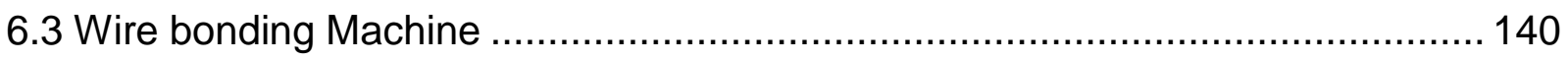

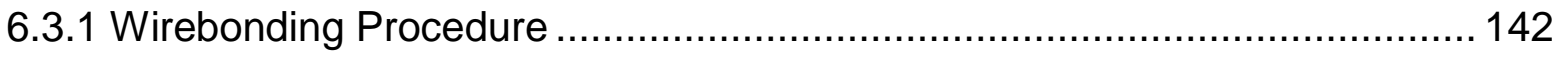

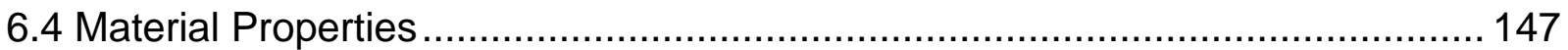

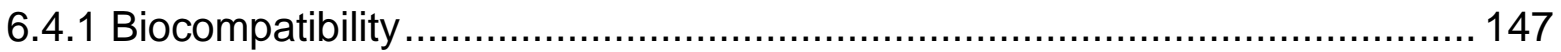

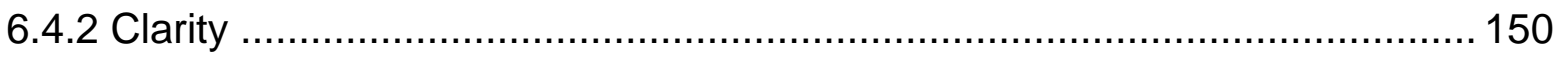

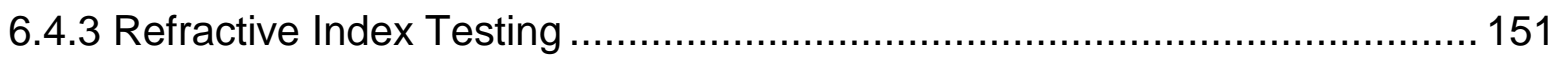

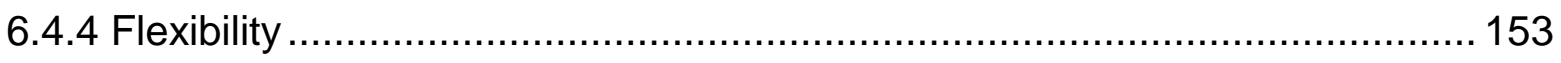

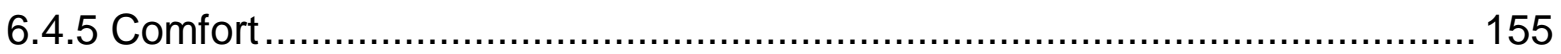

6.5 Power Dissipation and Bio-heat Transfer................................................... 157

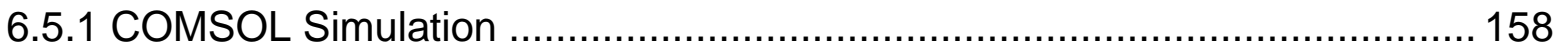

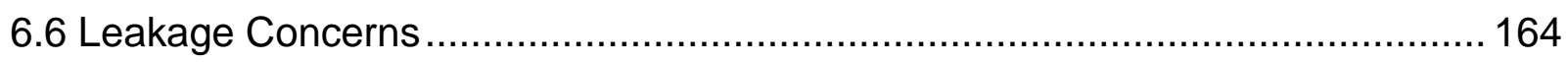

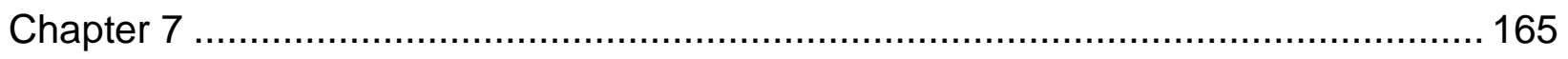

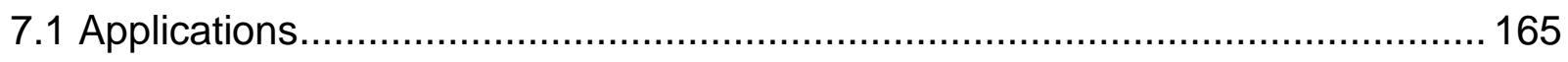

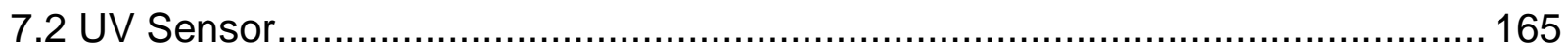

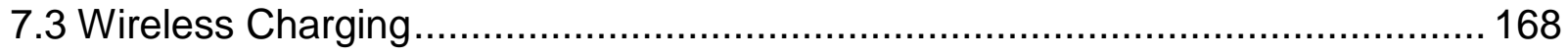

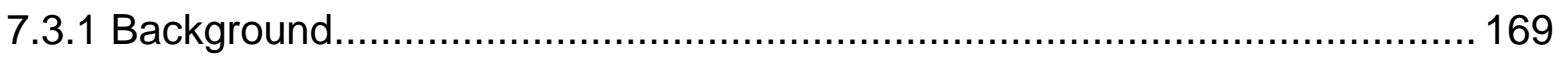

7.3.2 Rapid Prototyping a Charging Case .................................................... 172

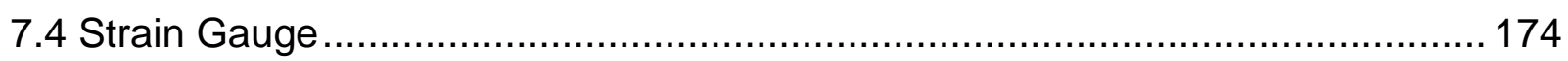

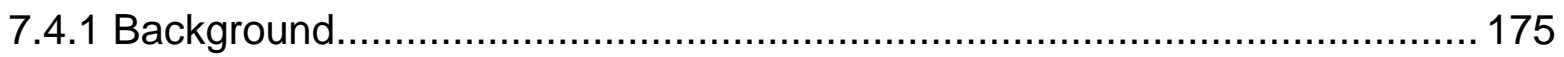

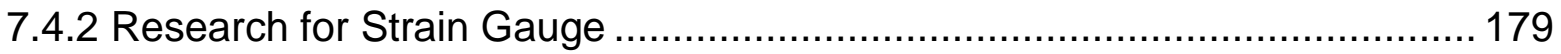

7.4.3 Design and Fabrication of Strain Gauge Molds and Masks ...................... 181

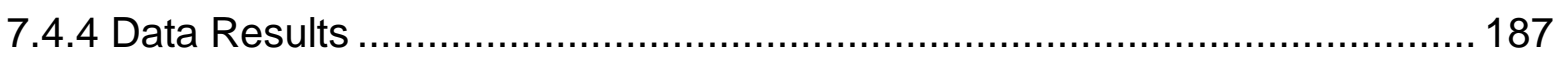




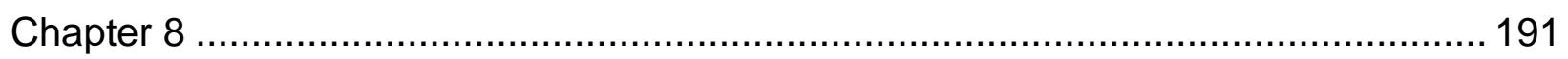

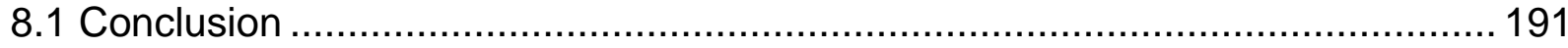

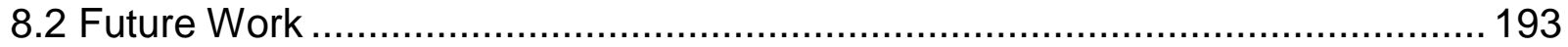

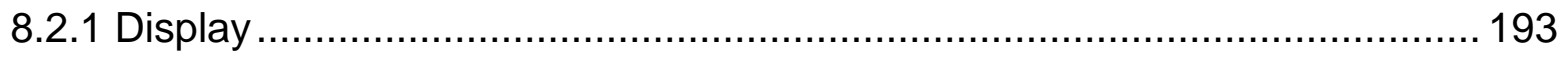

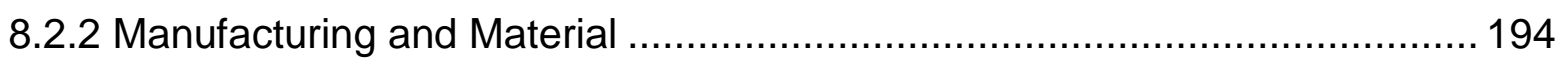

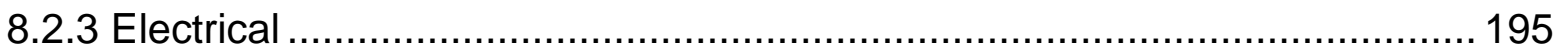

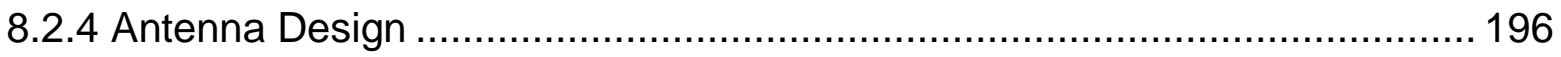

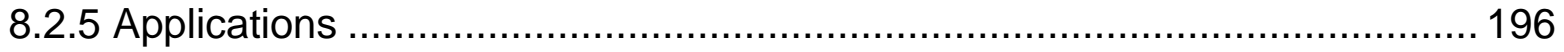

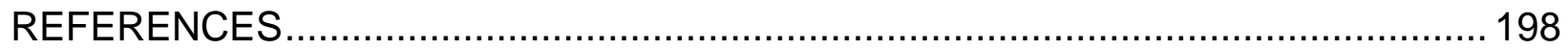
APPENDICES

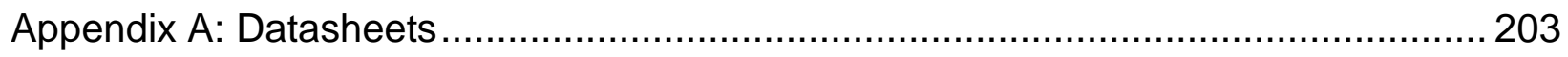

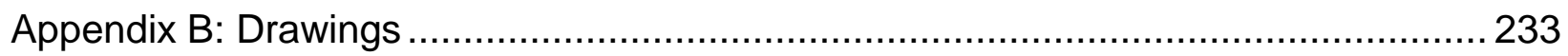

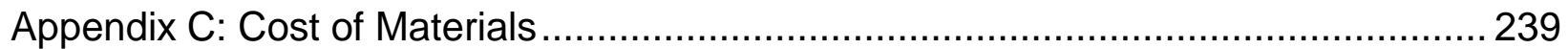

Appendix D: Gantt Chart for Research........................................................... 241 


\section{LIST OF TABLES}

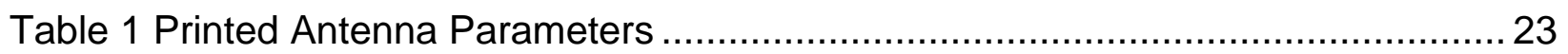

Table 2 Antenna Parameters Calculated from Reflection Coefficient............................ 79

Table 3 Measured Power Parameters for Efficiency Calculation................................... 92

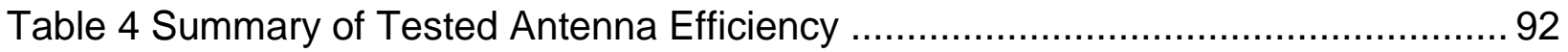

Table 5 First Iteration Coil Parameters.............................................................. 170

Table 6 Characteristics of Electrochromic Cells Made from Conducting Polymers .... 194 


\section{LIST OF FIGURES}

Figure 2.1 Active Contact Lens System Diagram with External Sensor ........................ 6

Figure 2.2 Active Contact Lens System Diagram ............................................... 7

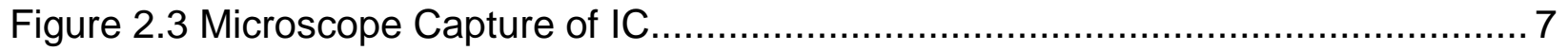

Figure 2.4 System Architecture of Custom Integrated Circuit..................................... 9

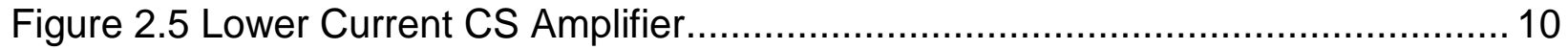

Figure 2.6 2.4GHz 3-Stage Voltage Multiplier ..................................................... 11

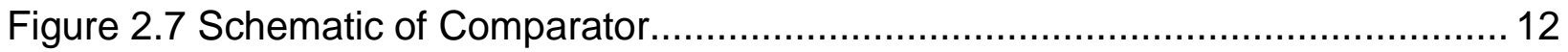

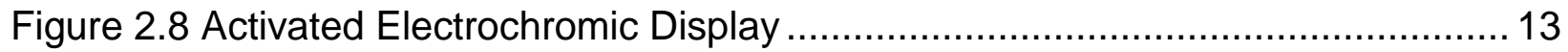

Figure 2.9 Enhanced image of charging circuitry ................................................. 14

Figure 2.10 Thin Film Lithium Ion Battery with Penny for Scale ................................. 15

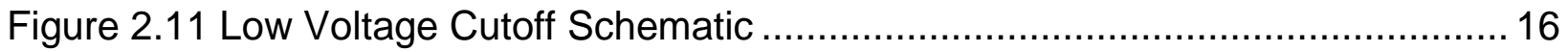

Figure 2.12 The wiring schematic of interconnections between device

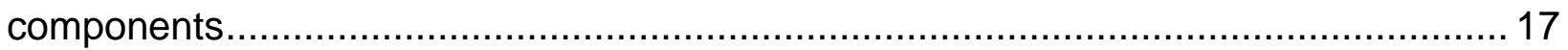

Figure 2.13 Exploded view of lens highlighting individual layers and components........ 18

Figure 2.14 Different Views of the Entire Contact Lens........................................... 19

Figure 3.1 Magnetic Field Generated by Single Loop .............................................. 21

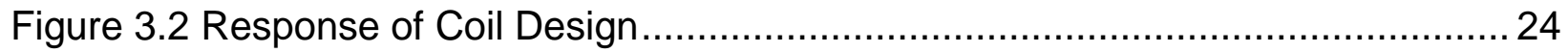

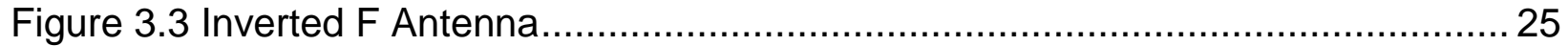

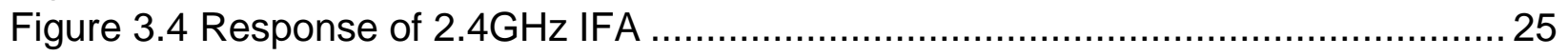

Figure 3.5 Curved IFA with Opened Ground Plane (top view) ….............................. 27

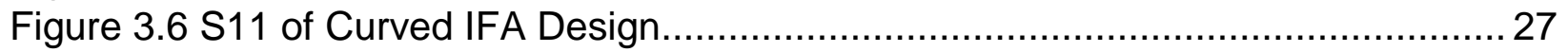

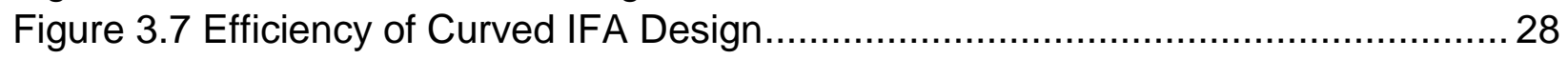

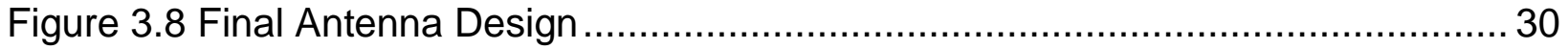

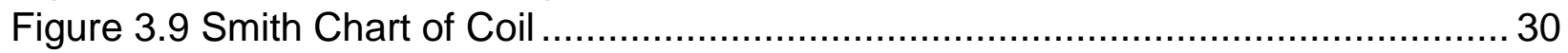

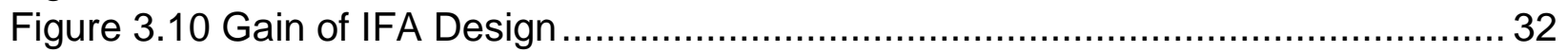

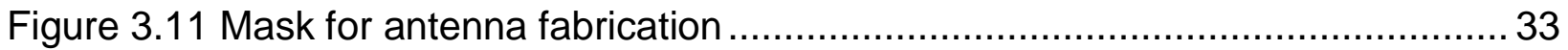

Figure 3.12 Cyrel Digital imaging machine printing antenna mask.............................. 34

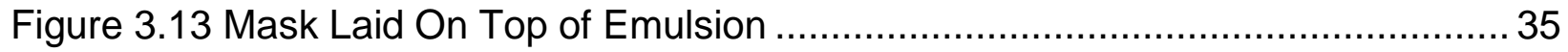

Figure 3.14 Removal of emulsion to create screen features ....................................... 36

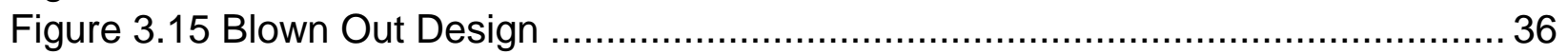

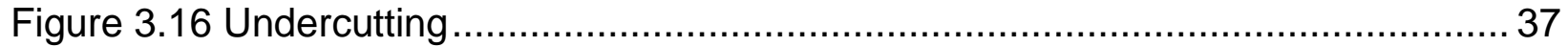

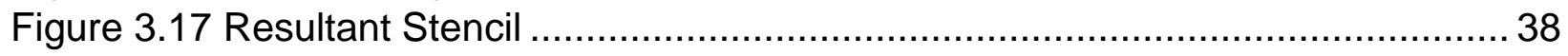

Figure 3.18 Application of the silver ink using the ATMAS machine ........................... 39

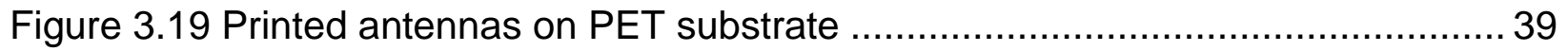

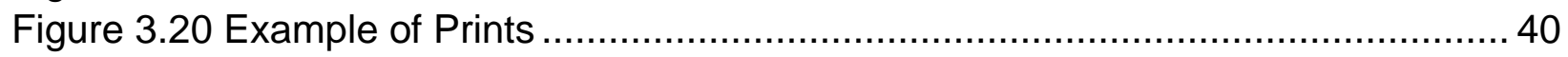

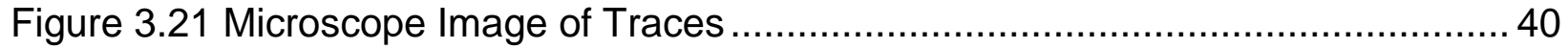

Figure 3.22 Example of antenna printing verification ............................................ 41

Figure 3.23 Interferometer Data of Printed Trace Sample ......................................... 42

Figure 3.24 Profilometer Data of Printed Trace Sample ................................................. 43

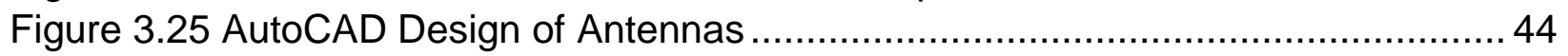

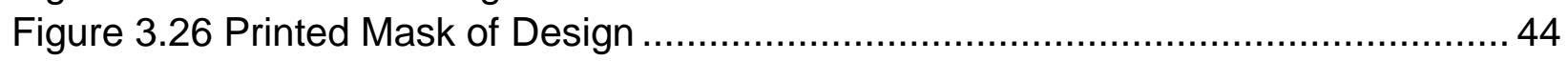


Figure 3.27 Completed Mask for Photolithography .................................................. 45

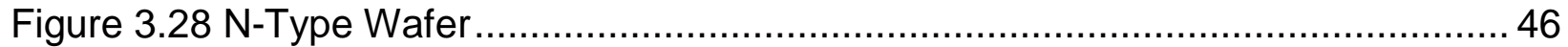

Figure 3.29 Base Solution and Curing Agent for PDMS …..................................... 47

Figure 3.30 Mixture of Base and Curing Agent Solution in Cup ................................. 48

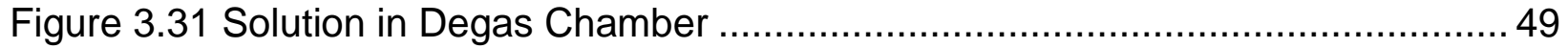

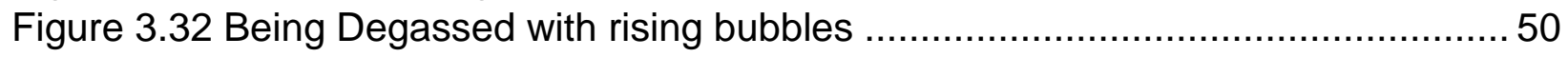

Figure 3.33 Aluminum Lined Spin Coater.............................................................. 51

Figure 3.34 PDMS-PET Start Substrate for Processing the Contact Lens ................... 52

Figure 3.35 Gas Tanks Need for Sputtering Process ............................................. 53

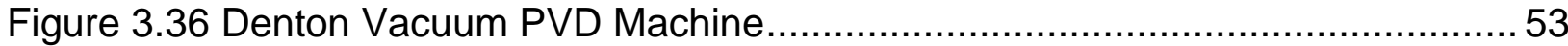

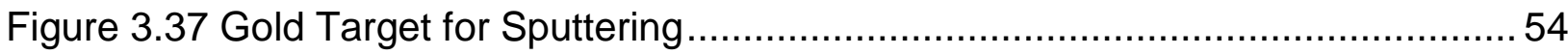

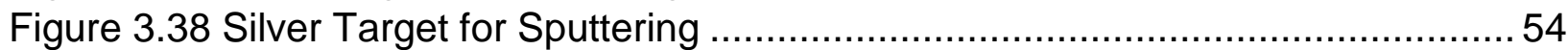

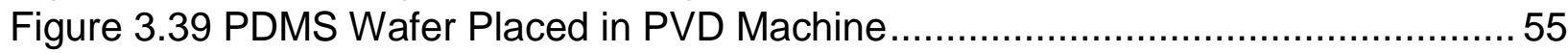

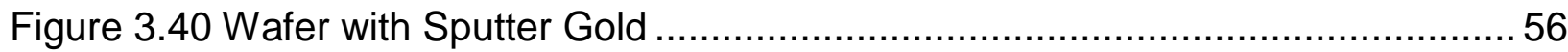

Figure 3.41 Positive Resist Spin Coater Machine ...............................................5 57

Figure 3.42 Wafer Sandwiched between Mask and Glass Slide Backing ....................58

Figure 3.43 Canon Aligner for Photolithography ................................................5 58

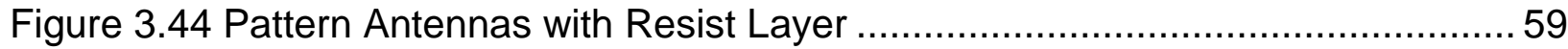

Figure 3.45 Patterned Gold 2.4 GHz/13.56MHz Antennas........................................ 60

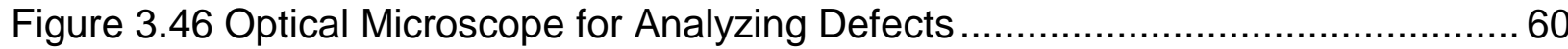

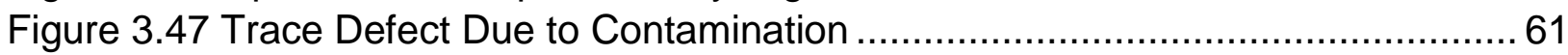

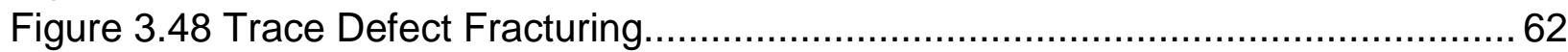

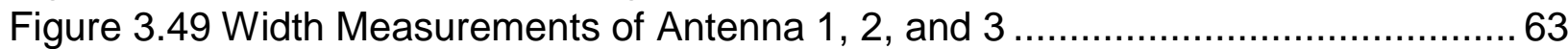

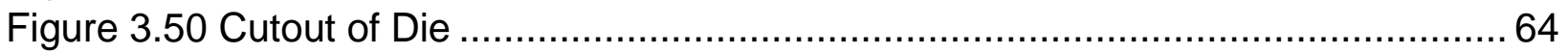

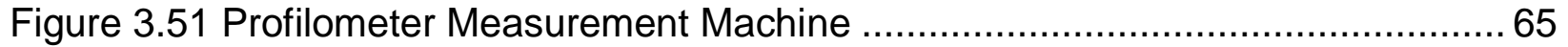

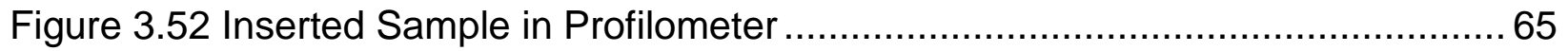

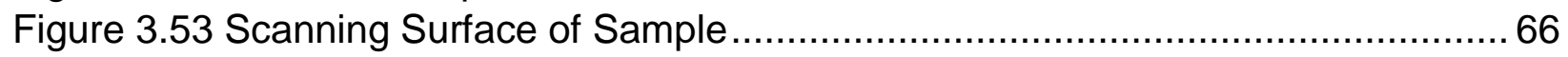

Figure 3.54 Results from Profilometer Scan Distance 5mm .....................................6 67

Figure 3.55 Results from Profilometer Scan Distance 3mm ......................................6 68

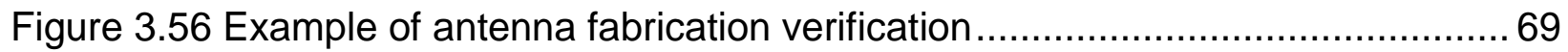

Figure 3.57 First Antenna Design, Consisting of One Solid Loop of Uniform

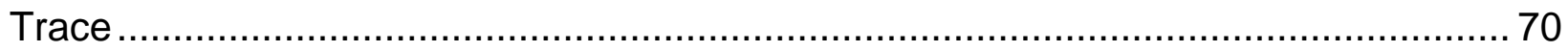

Figure 3.58 Second Antenna Design, Two Separate Loops of Uniform Trace

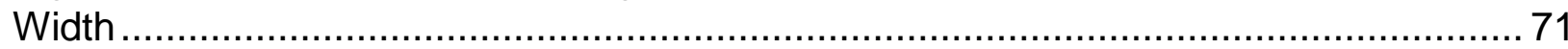

Figure 3.59 Third Antenna Design, Two Separate Loops of Non-Uniform Trace

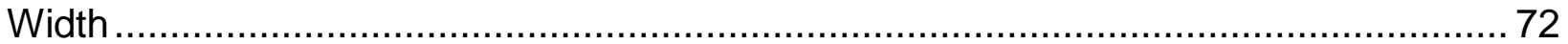

Figure 3.60 Antenna design 1 efficiency and power results .................................... 73

Figure 3.61 Antenna design 2 efficiency and power results ..................................... 73

Figure 3.62 Antenna design 3 efficiency and power results ..................................... 74

Figure 3.63 Antennas under testing. AUT1, AUT2, AUT3 from Left to Right..................76

Figure 3.64 Antennas under testing. AUT4, AUT5, AUT6 from Left to Right.................. 76

Figure 3.65 Antenna Gain Test Setup for $2.4 \mathrm{GHz}$ Far-Field Range of $50 \mathrm{~cm}$............... 77

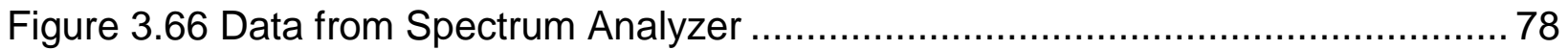


Figure 3.67 Impedance and Magnitude Response for AUT1 ...................................... 80

Figure 3.68 Impedance and Magnitude Response for AUT2 ……........................... 81

Figure 3.69 Impedance and Magnitude Response for AUT3 …................................ 82

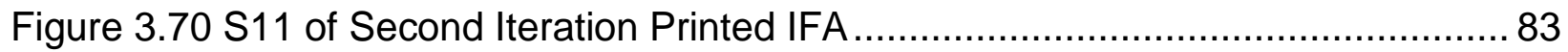

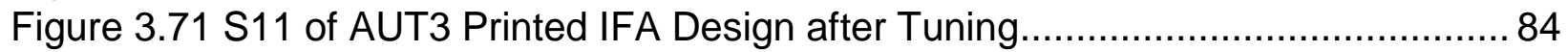

Figure 3.72 S11 of Second Iteration AUT1 Printed Coil Design .................................. 84

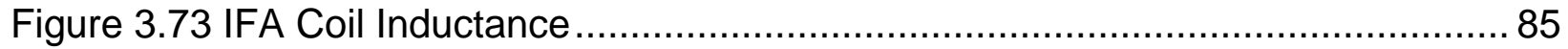

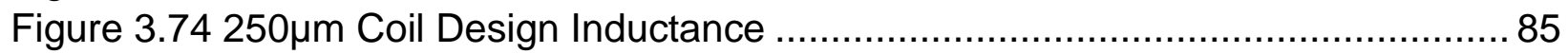

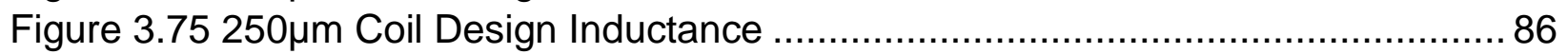

Figure 3.76 The AUT is rotated around the Horizontal Axis for Polarization

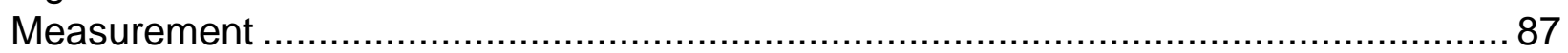

Figure 3.77 First Attempt Setup for Measuring Radiation Pattern .............................. 88

Figure 3.78 Resolved Setup for Measuring Radiation Pattern ................................... 88

Figure 3.79 AUT1 Radiation Pattern and Measured Received Power ......................... 89

Figure 3.80 AUT2 Radiation Pattern and Measured Received Power ......................... 90

Figure 3.81 AUT3 Radiation Pattern and Measured Received Power .......................... 91

Figure 3.82 Received Signal Power at Spectrum Analyzer (IFA) ............................... 93

Figure 3.83 Received Signal Power at Spectrum Analyzer (Coil) .............................. 93

Figure 4.1 Thin Film Lithium Ion Battery with Penny for Scale .................................. 96

Figure 4.2 Steps for Synthesis of Graphene Film ................................................. 101

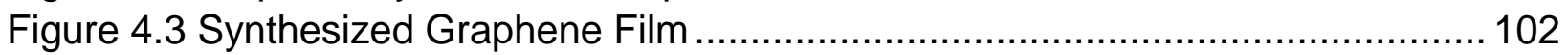

Figure 5.1 Stages of Operation for Electrochromic Display .................................... 104

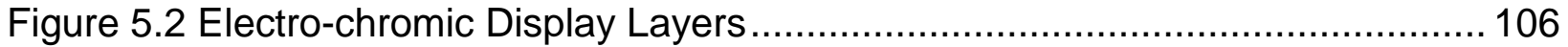

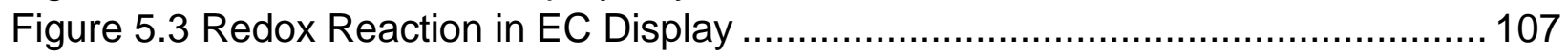

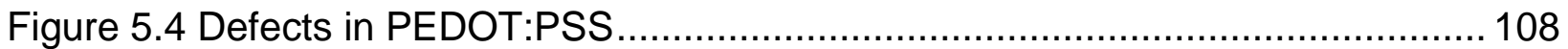

Figure 5.5 Color contrast $(\Delta E)$ and lightness $\left(L^{*}\right)$ vs. the PEDOT:PSS thickness

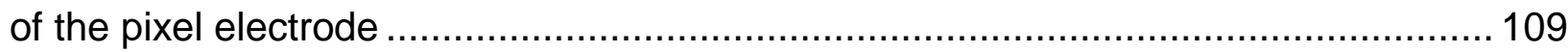

Figure 5.6 Spin Coater used to Create PEDOT Film........................................... 110

Figure 5.7 Results of a 1:1 ration of PEDOT and Methanol ....................................110

Figure 5.8 Optical Microscope Verification of Uniformity in Film ..............................111

Figure 5.9 4-Point Probe Used to Measure Resistance ......................................... 112

Figure 5.10 Chemistry Setup for Synthesis of PMMA …...................................... 115

Figure 5.11 PMMA collected via vacuum filtration..................................................116

Figure 5.12 Compounds Needed for Electrolyte Mixture ..........................................117

Figure 5.13 Final product of mixed compound polymer electrolyte ...........................117

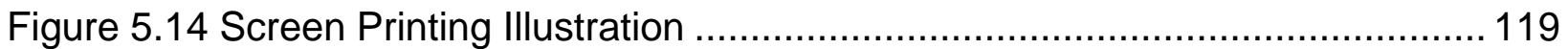

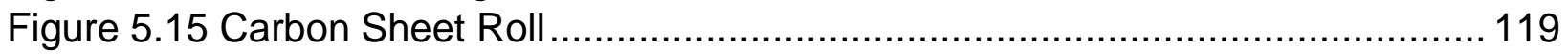

Figure 5.16 Feeding Carbon Sheet into Cyrel Digital Imager .................................. 120

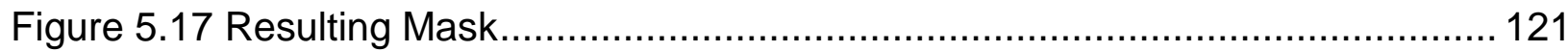

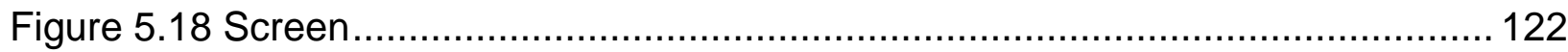

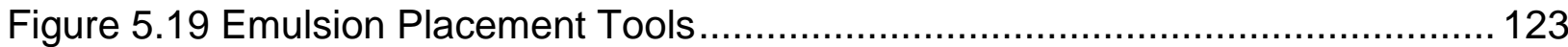

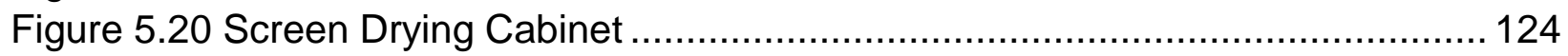

Figure 5.21 Mask Laid On Top of Emulsion ...................................................... 124 


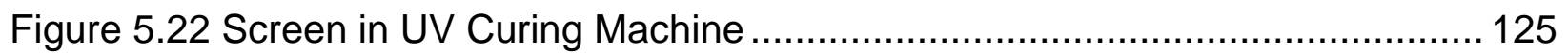

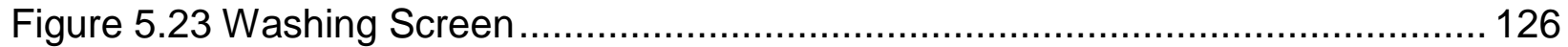

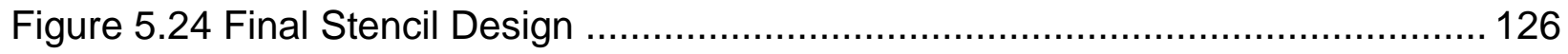

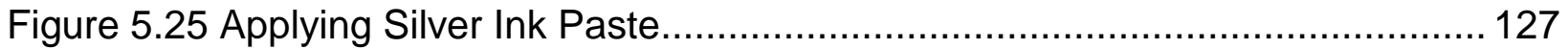

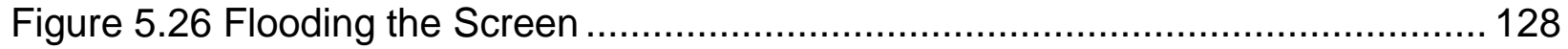

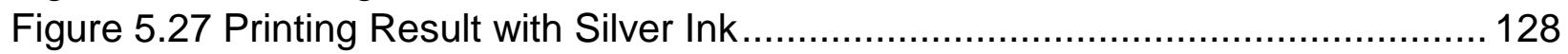

Figure 5.28 PEDOT:PSS and Electrolyte Material .............................................. 129

Figure 5.29 Final Print of Silver Ink, PEDOT:PSS, and Electrolyte ............................ 129

Figure 5.30 Resulting Stages from applying a 1 Volt Potential................................. 130

Figure 6.1 PET lens base completely encapsulated by PDMS …........................... 131

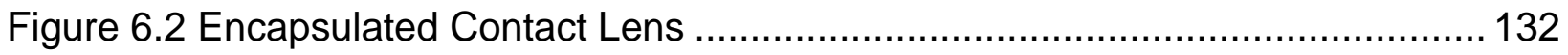

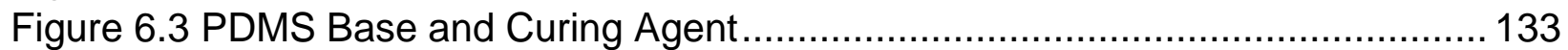

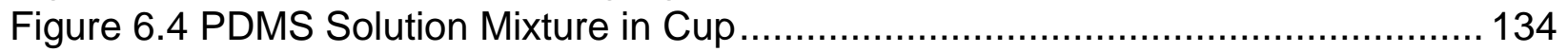

Figure 6.5 Mixture Inserted in Degas Chamber................................................... 135

Figure 6.6 Chamber Vacuum Engaged with Rising Bubbles in Mixture ..................... 136

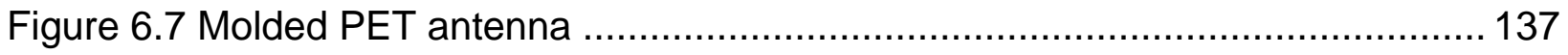

Figure 6.8 Pouring of PDMS to form external lens layer ........................................ 139

Figure 6.9 Bonding Configuration for IFA Antenna............................................. 141

Figure 6.10 Bonding Configuration for Coil Antenna ............................................ 141

Figure 6.11 Manually Operated Wedge Bonder 5432 Machine................................. 142

Figure 6.12 Diagram of Bonding Wire to Die Pad ................................................. 143

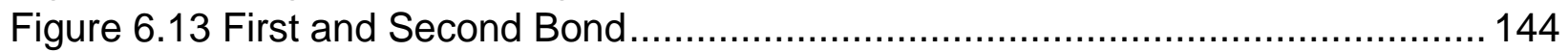

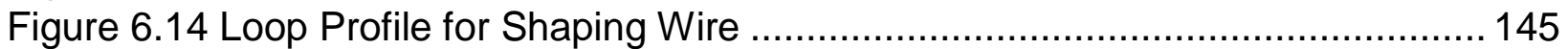

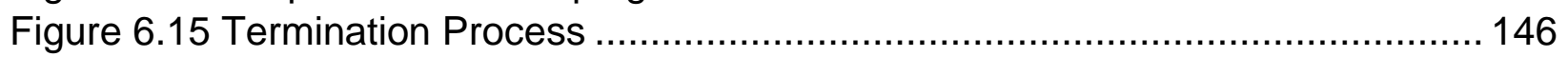

Figure 6.16 Coupon Used for Calibration ........................................................... 147

Figure 6.17 Materials used in designing refractive index test fixture .......................... 152

Figure 6.18 Contact angle test in progress using VCA Optima system ...................... 155

Figure 6.19 Bar chart of average contact angles for PET and PDMS, including

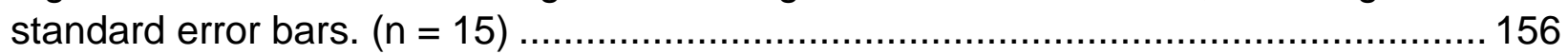

Figure 6.20 Proposed Finite Element Sketch of Human Eye ................................... 159

Figure 6.21 Axisymmetric COMSOL Sketch of the Human Eye ................................ 160

Figure 6.22 3D Surface Temperature Distribution after 30 Second Exposure-

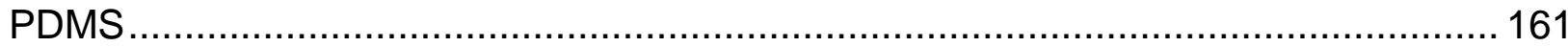

Figure 6.23 2D Surface Temperature Distribution after 30 Second Exposure-

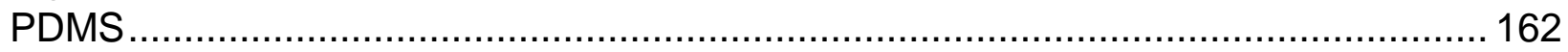

Figure 6.24 3D Temperature Distribution after 30 Second Exposure-PET ................. 162

Figure 6.25 2D Surface Temperature Distribution after 30 Second Exposure-

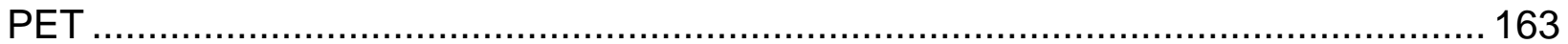

Figure 7.1 External UV Sensor System Diagram ................................................ 166

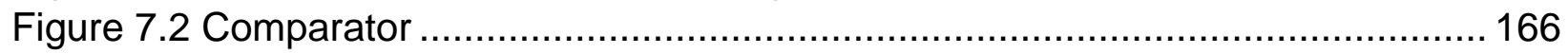

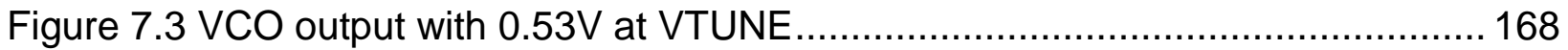

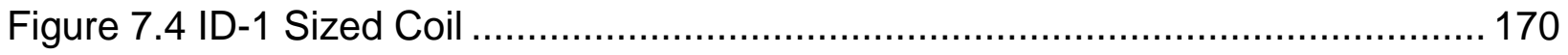

Figure 7.5 Simulation Results of Printed Coil ................................................... 171 


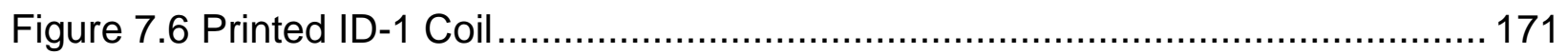

Figure 7.7 Oscilloscope Capture of Tx Signal (top) and Rx Signal (bottom) ............... 172

Figure 7.8 Rapid Prototyped Box Case for Inductor Transmit Coil.............................. 173

Figure 7.9 Inductor Coil with Driving Circuit and Power Source ................................ 174

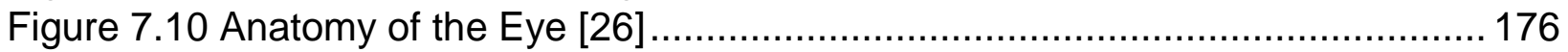

Figure 7.11 Flow pathway of aqueous humor through anterior chambers [26]........... 177

Figure 7.12 Progression of high-pressure glaucoma [28] .................................... 178

Figure 7.13 Example Strain Gage Design ......................................................... 180

Figure 7.14 Strain Gage Mold (left) and Mask (right), Laser-cut from PET ................ 181

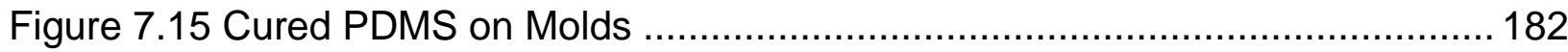

Figure 7.16 Denton Vacuum PVD Machine ...................................................... 183

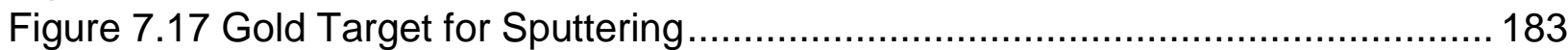

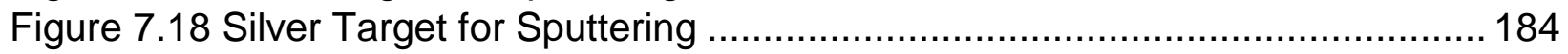

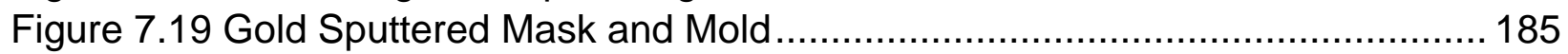

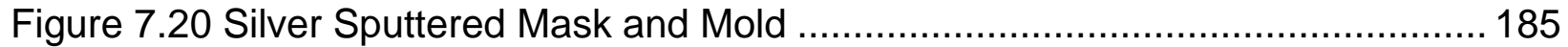

Figure 7.21 Gold and Silver Strain Gages, First Production................................... 185

Figure 7.22 Microscope Capture of Strain Gauge Gold Sample .............................. 186

Figure 7.23 Silver Strain Gage with Silver Epoxy Connectors, $50 \Omega$ Impedance ......... 187

Figure 7.24 Wheatstone Bridge Circuit, Active and Passive Resistors ....................... 188

Figure 7.25 Final Testing Circuitry and Schematic .............................................. 189

Figure 7.26 Wheatstone Bridge Measurement, Gage Resistance v. Vm ................... 189 


\section{Chapter 1}

\subsection{Introduction}

As we enter the age of transparent and flexible electronics, new devices that were once thought unfeasible are now becoming a reality. Research into the area of clear active contact lenses demonstrates the power of this new technology, with such applications as Google's new devices that promise to continuously detect glucose levels in the eye [1]-[3]. Research into active contact lenses has seen a recent surge in popularity in both the public spheres and academic where Cal Poly is working to be a strong player in this area of research and development of clear active contact lenses. As this research has progressed, it has expanded to include a power source design, a transparent display, and new fabrication techniques. The addition of these new devices to the lens have two purposes. The first was to create a more robust device design and the second was to develop a stand-alone multi-sensing system. The prototype built in 2013 concentrated on the system level details of an active contact lens and successfully received (wireless) power from an external wireless transmitting source [4]. The goal of this project is to build and improve on that infrastructure by scaling down the current antenna receiver design, adding a custom IC to process the data and power management in order to charge an on-lens battery, a battery/super capacitor as a power source, an electrochromic display for visually displaying the data to the user, as well as two types of sensors (one on-lens and off-lens) as a driver to test the functionality of the lens system. 
Successfully implementing these new features on a contact lens, opens up countless potential applications for this system. Some examples are: sensing glucose or drug level from eye tears, communicating information with nearby lenses or devices, assistive technology for deaf people, drug dispersion system, etc. The focus of this research compared to the Cal Poly's prior initial 2013 prototype, has not changed, but rather undergone an expansion to make an accurate scale model, implementing an infrastructure in place for receiving power, data, and display, as well as address biocompatibility concerns. Collectively, the original members, myself included, have designed and fabricated micro-feature electronic circuits and encapsulated them in a substrate suitable for a contact lens[4]-[6].

The 2013 prototype design for the circuit entailed manufacturing a flat lens with the embedded circuitry. However, during the research and development process, the team decided that the ability to manufacture a curved lens with intact circuitry was essential to the mission. This challenge rendered our traditional power source and surface mount components obsolete, as they were relatively large, rigid, and unable to flex with the lens. In order to resolve this, design and fabrication of flexible micro-components and a flexible rechargeable power source were implemented, specifically for the power source a thin film super capacitor and battery. I took on this challenge, and worked with the following departments to successfully integrate this device: nanofabrication facilities at University of California, Santa Barbara, the microfabrication facility at Cal Poly, graphic communication facility at Cal Poly, and the polymers lab at Cal 
Poly. Accompanied by a recharge circuit, the power source that I developed drives the indicator electro-chromic ink display and a wireless receiver. An external system consisting of a sensor, and wireless transmission circuitry was used to signal the lens during an event. Creating devices that are flexible enough to be shaped and that would actually work on a curved lens was one challenge, but actually shaping the lens also turned out to be a major challenge. A modified approach was developed and analyzed for shaping a lens of $1 \mathrm{~cm}$ diameter to a radius of curvature of $7.8 \mathrm{~mm}$. It has been previously shown by Otis et. all, at the University of Washington that a circuit can be developed and molded into a curved lens using existing microfabrication technology[5]. Dr. Otis's process involved heating the mold up to $180^{\circ} \mathrm{C}$ and pressing the substrate into it to develop a smooth curvature. The problem presented by this technique is that our current conductive ink has a flash point much lower than $180^{\circ} \mathrm{C}$, meaning that it will likely catch fire during the manufacturing process and render the device unusable. To overcome this manufacturing challenge an injection mold method was combined with Dr. Otis's process to encapsulate and mold the lens at a lower temperature $\left(80^{\circ} \mathrm{C}\right)$.

Two types of materials (PET and PDMS) were experimented with as the substrate which unfortunately needed two different types of manufacturing processes. The first method utilized a printing lab working with Dr. Keif of Cal Poly's Graphic Arts department to modify the current methods of screen-printing, an alternative method of screen printing was approached to manufacture a layer by layer power source as well as a planar antenna on a flexible transparent 
substrate. I feel a high value in manufacturing this device using the screenprinting technology. The method of screen-printing a circuit is valuable because of its versatility, rapid turnover time, and low cost. Printed electronics allows us to develop a wide variety of transparent and flexible substrates - ideal for this application. In addition, screen-printing is an additive technique, as compared to etching used in current PCB fabrication, which allows for multi-layer designs without the use of harmful etchants. With the current design constraints however, I felt it was important to pursue more documented avenues to ensure that we demonstrate the viability of our device design.

This led to a second approach which involved a change in the manufacturing process for the antenna traces and the substrate (PDMS). With the help of Dr. Savage, I used the Micro-Fabrication lab facilities to utilize a method known as physical vapor deposition of metal onto the PDMS substrate. The sputtering and etching process are well documented, with an in-depth student and faculty member knowledge base with which to draw from. For these reasons I felt that pursuing this avenue was vital to the success of the devices.

This thesis describes design, fabrication, and encapsulation techniques for the notification system embedded in a contact lens. Chapter 1 is the introduction that explains different research grasp attempts of the prior 2013 prototype, fabrication the lens, and the addition of new components and features in the second prototype that make the lens superior over the 2013 prototype. Chapter 2 describes an overview of the entire system and functionality of the subsystems. Chapter 3 presents the theory and fabrication of planar antennas 
on PDMS and PET, compares scatter parameters of the PET and PDMS designed. Chapter 4 describes the research and development of graphene, its application as a power source with an ionic liquid and gel separator as well as potentiostat test results. Chapter 5 explains the electro-chemical reactions behind the ink display, design methods, manufacturing, and testing of the device. Chapter 6 describes PDMS properties, encapsulation process, biocompatibility, and power leakage concerns. Chapter 7 describes different application of the lens and analyzes an alternative driving sensor describing theory and the relation of displacement to eye pressure. This chapter also explains the fabrication process and testing of the sensor. The final section of this thesis, Chapter 8, encompasses the conclusion of the subsystem designs of the active contact lens and presents future research avenues to pursue in order to solve current challenges with the lens. 


\section{Chapter 2}

\subsection{System Level Design of Lens}

Our 2013 prototype goal was to develop an infrastructure that future contact lens projects could use. We needed a driver application to design to and we picked a system that would alert a user if the UV level rose above what is considered a safe level (see Chapter 7.2). In this first prototype was used to test the power management system, data receiver, power receiver, and electrochromic ink display. A second driver application was added for this thesis in order to look at on-lens sensors. The second on-lens application is a strain gauge sensor which would be used to test for symptoms of Glaucoma (See Chapter 7.4). In the case of the UV threshold detector, the benefits of an external sensor over an integrated one include the option to implement other types of sensors and reducing the area required for the circuitry embedded in the lens. The capabilities of a modular system was the inspiration for having the sensor outside of the lens. The type of sensor can easily changed for another type of sensor depending on the needs of the users' environment.

Figure 2.1 shows the system block diagram of the transmitting, receiving, and processing systems.
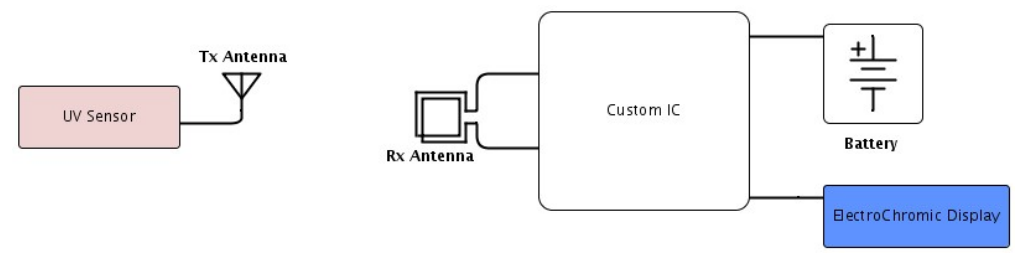

Figure 2.1 Active Contact Lens System Diagram with External Sensor When the external sensor receive the appropriate threshold conditions, it will transmit a 2.4GHz signal out from an antenna where it will be received by the printed/microfabricated antenna embedded in the contact lens. The purpose of the embedded 
antenna is to receive the wireless signal transmitted by an external sensor or inductively charge the onsite battery. The two modes of operation for the antenna are that it will act as a receive antenna for a $2.4 \mathrm{GHz}$ wireless signal and perform as the tag coil for a $13.56 \mathrm{MHz}$ charging voltage (see Chapter 3 ). A custom chip manages the recharge of the battery along with amplifying the $2.4 \mathrm{GHz}$ wireless signal. A system level diagram of the lens is shown in Figure 2.2.

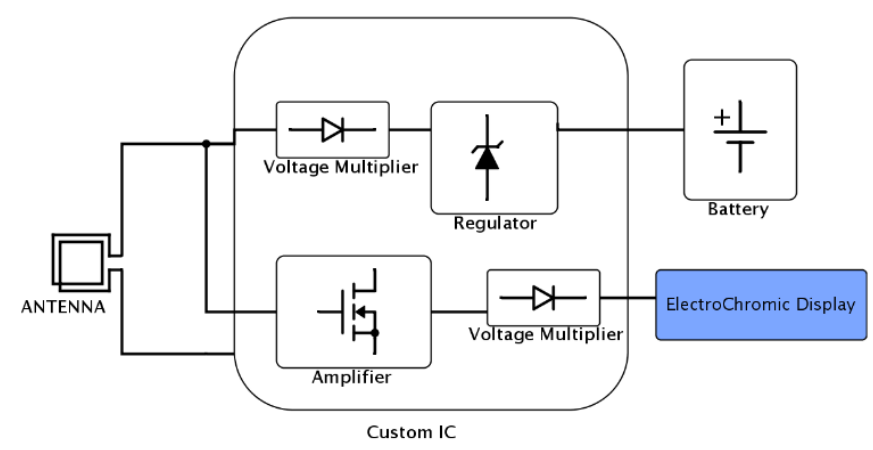

Figure 2.2 Active Contact Lens System Diagram

The main functionalities of the contact lens are handled by a custom designed integrated circuit (IC) shown in Figure 2.3.

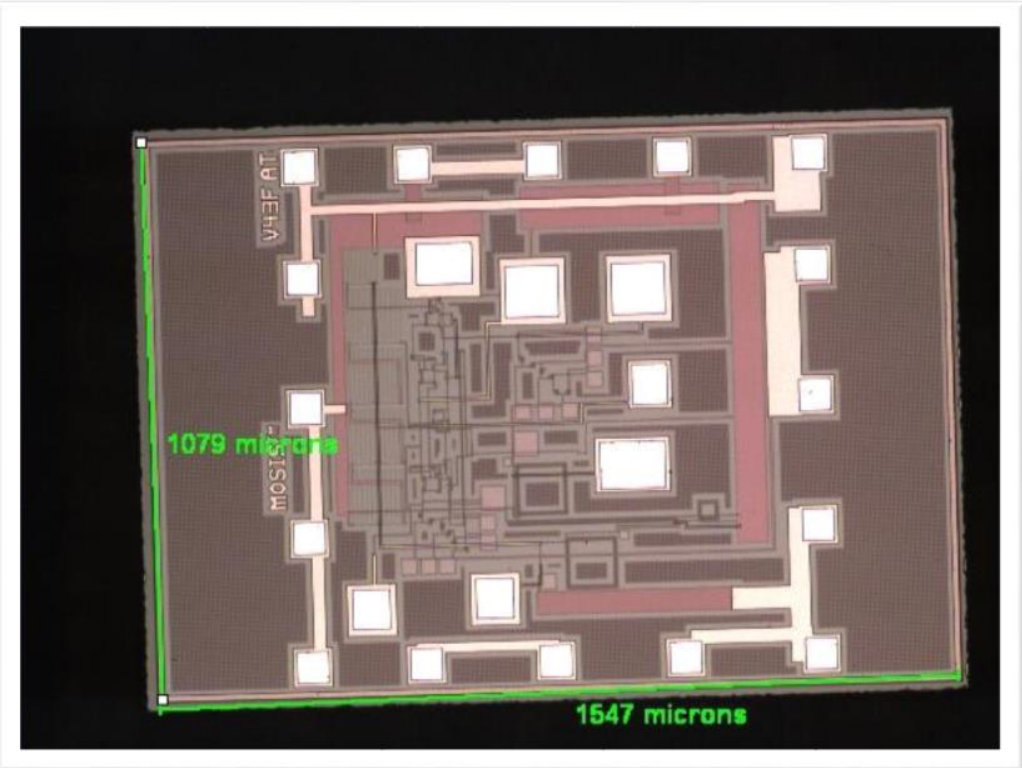

Figure 2.3 Microscope Capture of IC 
These functions include: detect incoming signal from the external sensor circuit, handle inductive recharging, manage battery voltage level, and drive the display. Additionally, the IC must have a total current draw of $5 \mu$ amps in order to achieve an operation time of an hour running off the $5 \mu \mathrm{Ah}$ battery (see Chapter 4 ). The IC was designed through Cadence Virtuoso and fabricated using the ON Semiconductor CMOS C5 0.6um process. Figure 2.4 shows the internal subsystems that make up the IC. 


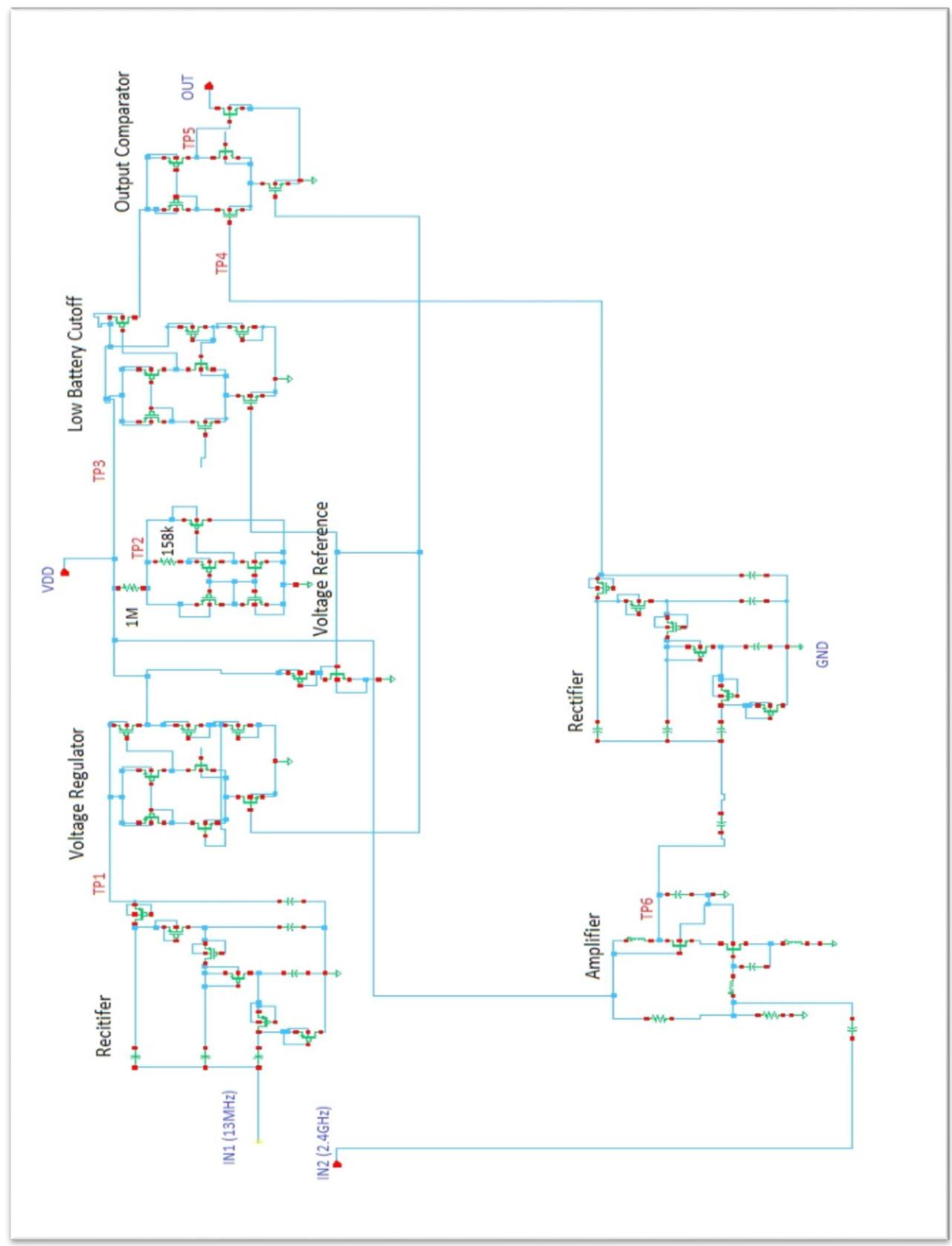

Figure 2.4 System Architecture of Custom Integrated Circuit 
From the system architecture it is shown that there are two paths a signal can take. I will first start describing the lower path for IN2. When $2.4 \mathrm{GHz}$ is received from the antenna, the signal goes through an amplification stage. Figure 2.5 shows the common source design with inductor degeneration.

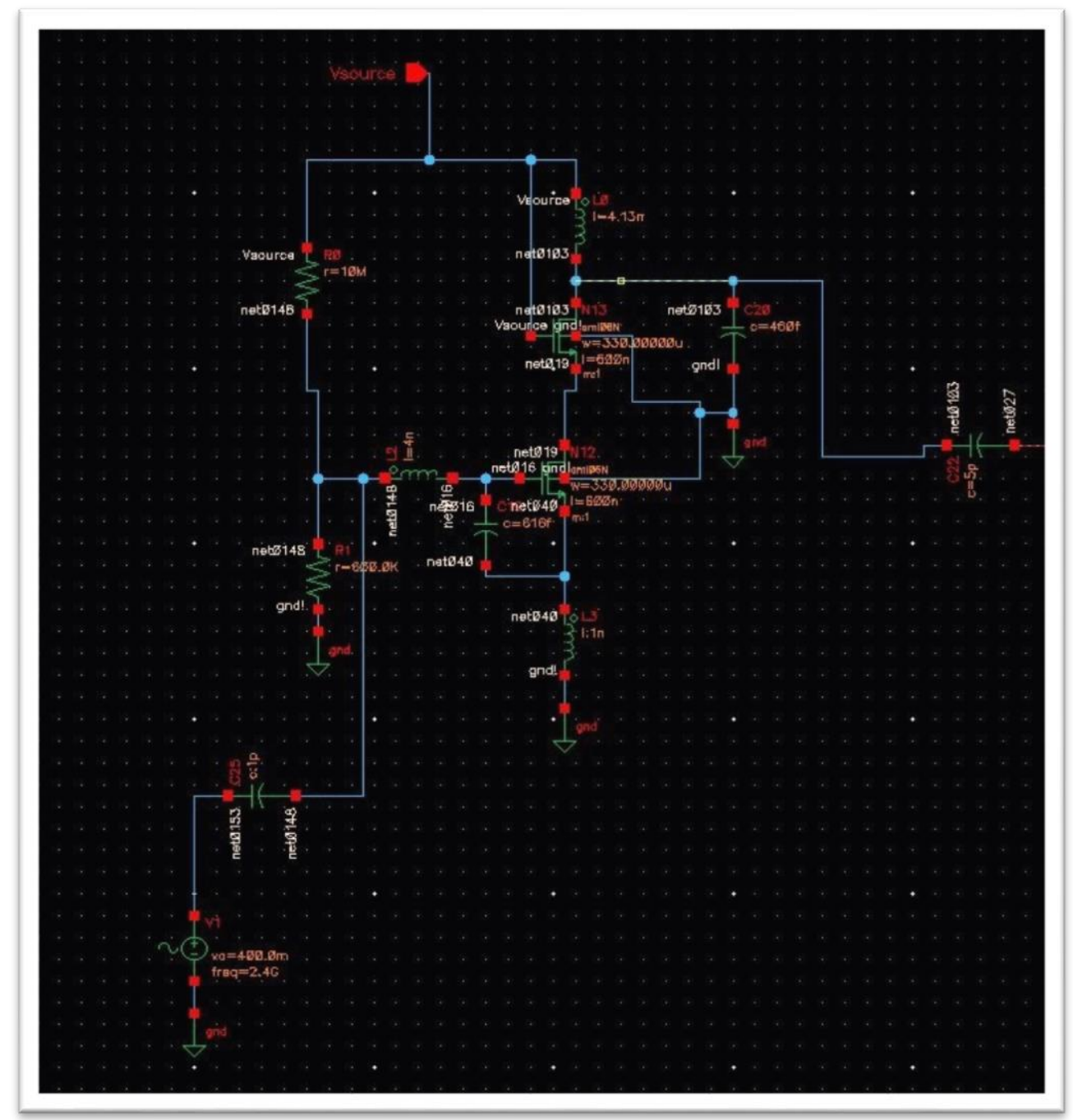

Figure 2.5 Lower Current CS Amplifier

The amplifier had two important requirements that needed to be met. It must be input matched to $50 \Omega$ at $2.4 \mathrm{GHz}$ and have a low quiescent current. The amplifier topology that was implemented was a common-source amplifier with inductor degeneration for power matching. An 18dBm signal power level must be present at the chip's input in 
order for the rectifier to operate. Since the original specification called for an operational distance of $30 \mathrm{~cm}$, the output power from the external sensor with a $2 \mathrm{dBi}$ antenna must be at least $45 \mathrm{dBm}$. That is an extremely large power so in order to reduce the output power requirement of the sensor, an amplifier was introduced at the input of the chip. Figure 2.5 is a modified common source topology where the design was adjusted to support a lower current draw. The gate bias resistive divider was changed in order to lower the gate to source voltage of the transistor.

The following subsystem that the signal passes through is a voltage multiplier that rectifies the signal. Figure 2.6 below shows a 3-stage voltage multiplier architecture.

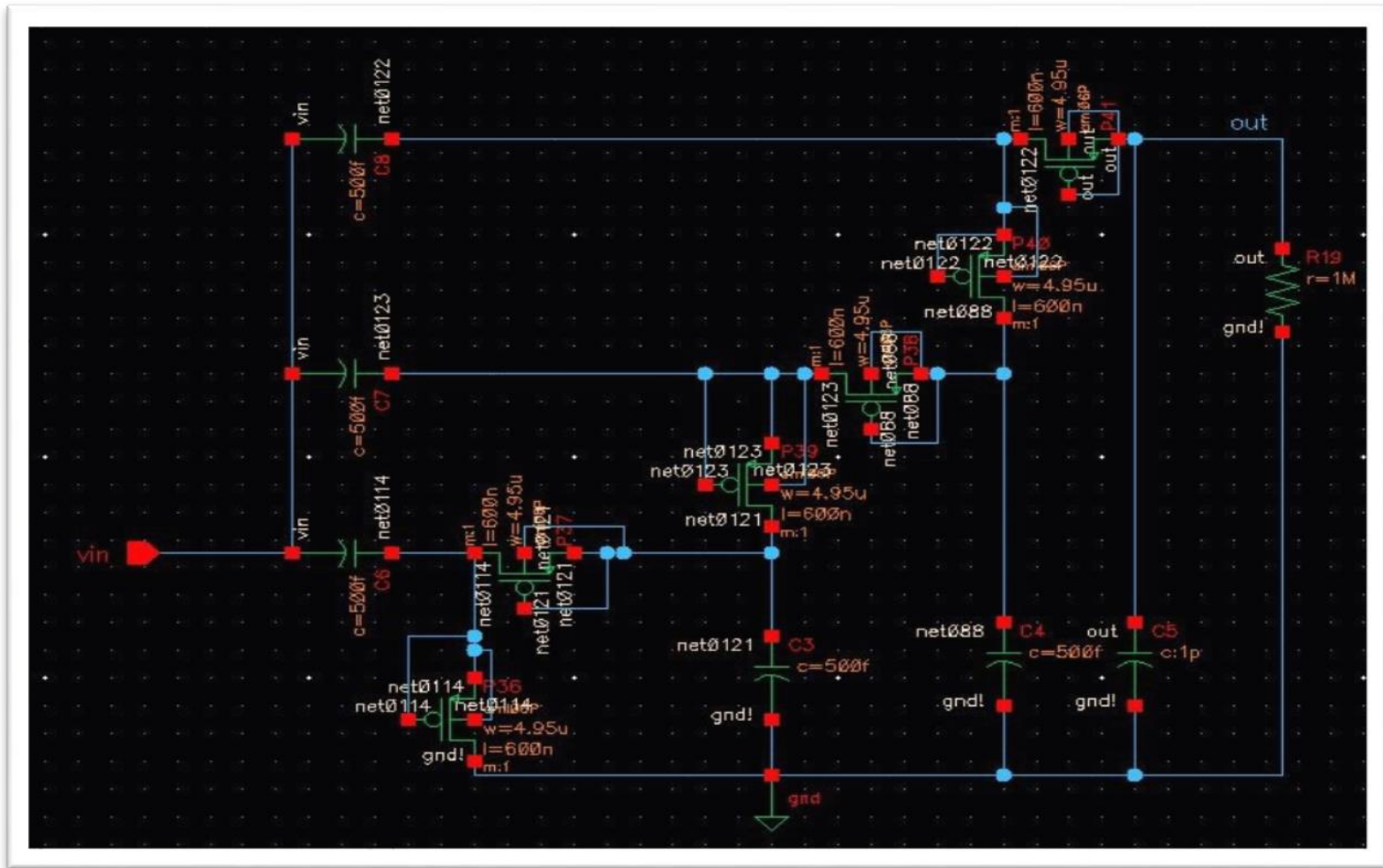

Figure 2.6 2.4GHz 3-Stage Voltage Multiplier 
The 3-stage voltage multiplier was designed with transistor dimensions $20 \mu \mathrm{m} / 6 \mu \mathrm{m}$ to keep channel resistances low. Since this rectifier is for a higher frequency signal, the capacitors were sized to be $500 \mathrm{fF}$ and an output storage capacitor of $1 \mathrm{pF}$. The $1 \mathrm{M} \Omega$ output resistance simulates the high input gate resistance of the interfaced comparator.

A comparator was designed to output a high value when the input is high. The comparator was implemented using MOSFETs for a differential pair. Figure 2.7 below shows the transistor architecture for the comparator.

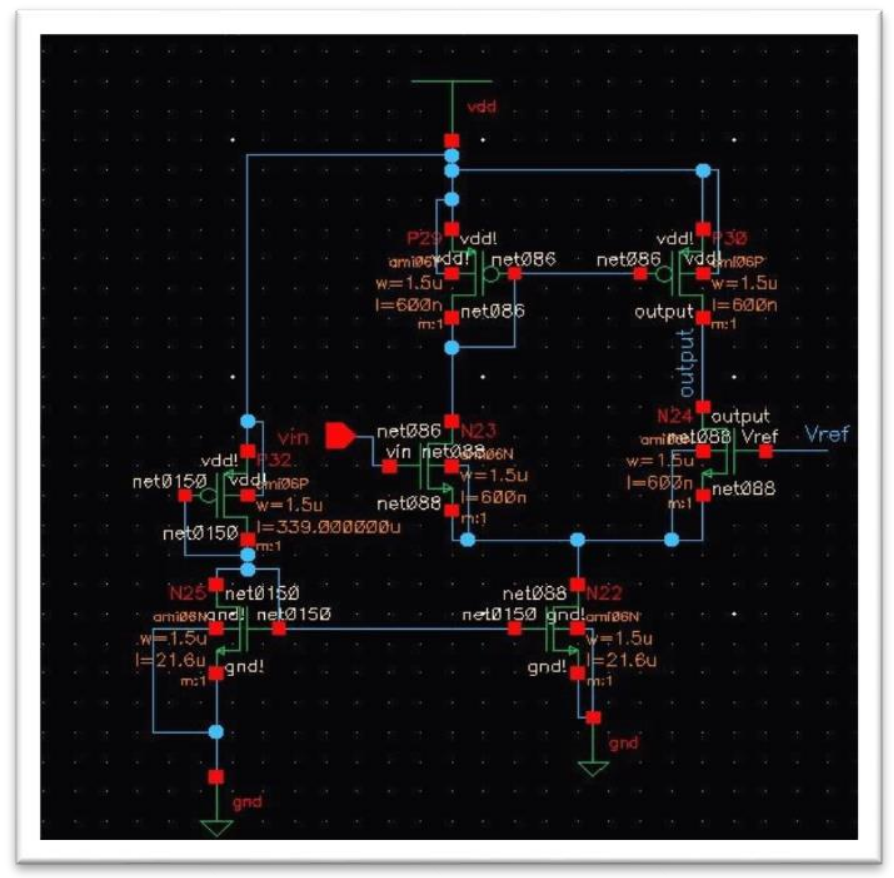

Figure 2.7 Schematic of Comparator

The tail current generated by N22 only turns on when the input voltage is high and sinks 99nA. When Vin is low, the tail current does not sink any current because of the active load generated by the PMOS transistors P29 and P30. When activated the output voltage would turn on the display indicating that a triggered event has occurred. The display interfaced with the chip is an electrochromic ink display that does not need 
constant current to function like an LED (see Chapter 5). The activated display can be seen in Figure 2.8.

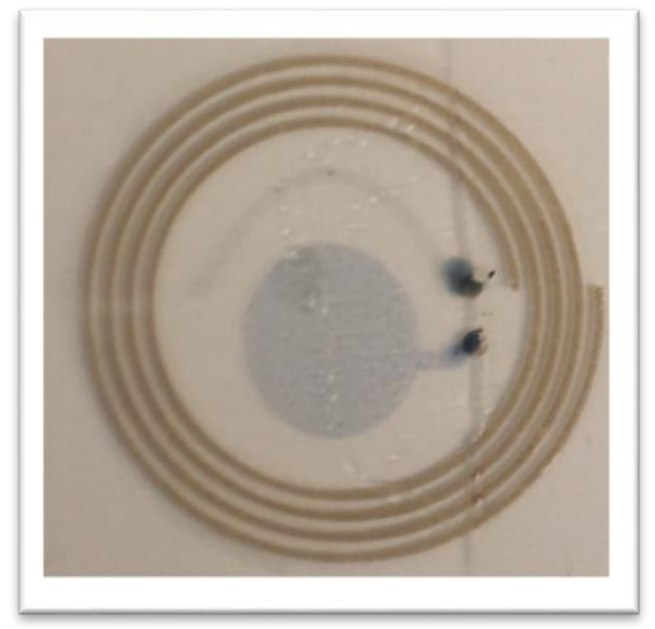

Figure 2.8 Activated Electrochromic Display

The circuitry must operate such that it is not damaged by the discharging of the power source and the radiofrequency transmission from the antenna. Therefore, protection circuitry was designed ensuring both regulated charging voltage and low battery voltage cut off to preserve battery longevity. This is the circuitry path IN1 of the IC schematic in Figure 2.4 shown prior in this chapter. IN1 initializes when a $13.56 \mathrm{MHz}$ frequency is coupled with the induction coil which enables near-field charge (NFC) of the device. The two red boxes indicate the protection circuitry in Figure 2.9. 


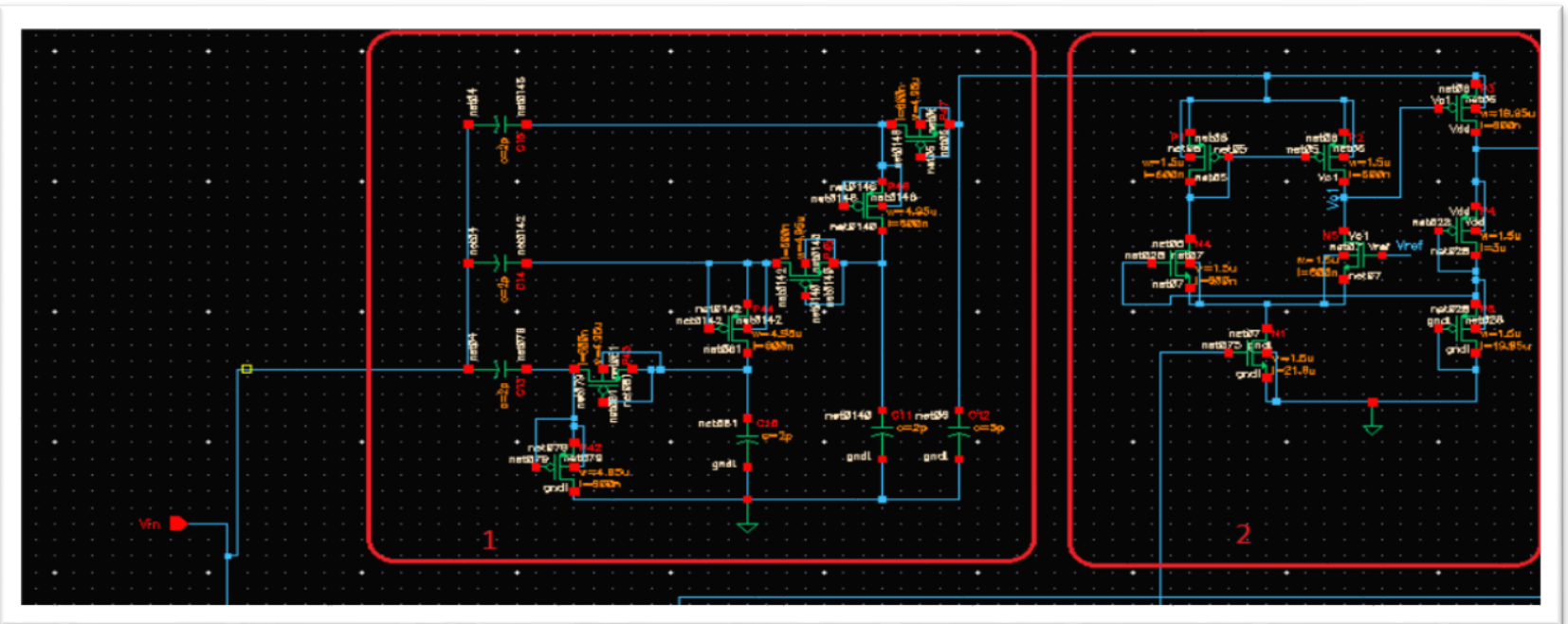

Figure 2.9 Enhanced image of charging circuitry

Red box one is a three stage charge pump that rectifies the input sinusoidal wave to ensure it matches specific requirements listed in the appendix. In red box two, a voltage regulator clamps the charging input voltage to 4.1 volts to ensure that the batter receives optimal voltage and is not damaged during charging.

The battery used for prototyping was a thin film battery that had a capacity of $5 \mu \mathrm{Ah}$. Figure 2.10 shows a reference scaled picture of the size of the thin film battery. 


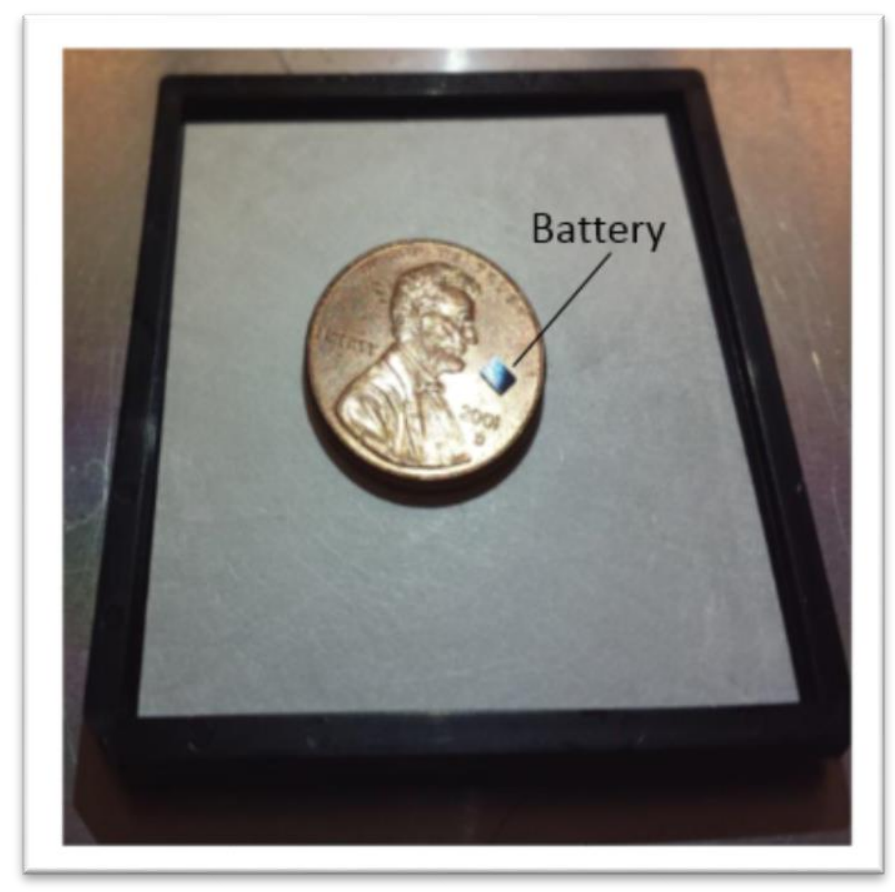

Figure 2.10 Thin Film Lithium Ion Battery with Penny for Scale Research was initiated to look at alternative power sources that had a greater energy density and flexible. Graphene supercapacitors were developed to be that alternative.

Deep discharge of the battery must also be prevented to maximize the lifetime of the battery. In accordance to $\mathrm{CBC} 005$ datasheet specifications, the battery must stop discharging when the voltage drops to $3 \mathrm{~V}$ or degrading of the cells will occur. A low battery cutoff switch was designed to prevent the battery from entering the forbidden discharge state. Figure 2.11 below shows the schematic of the low voltage cutoff circuitry. 


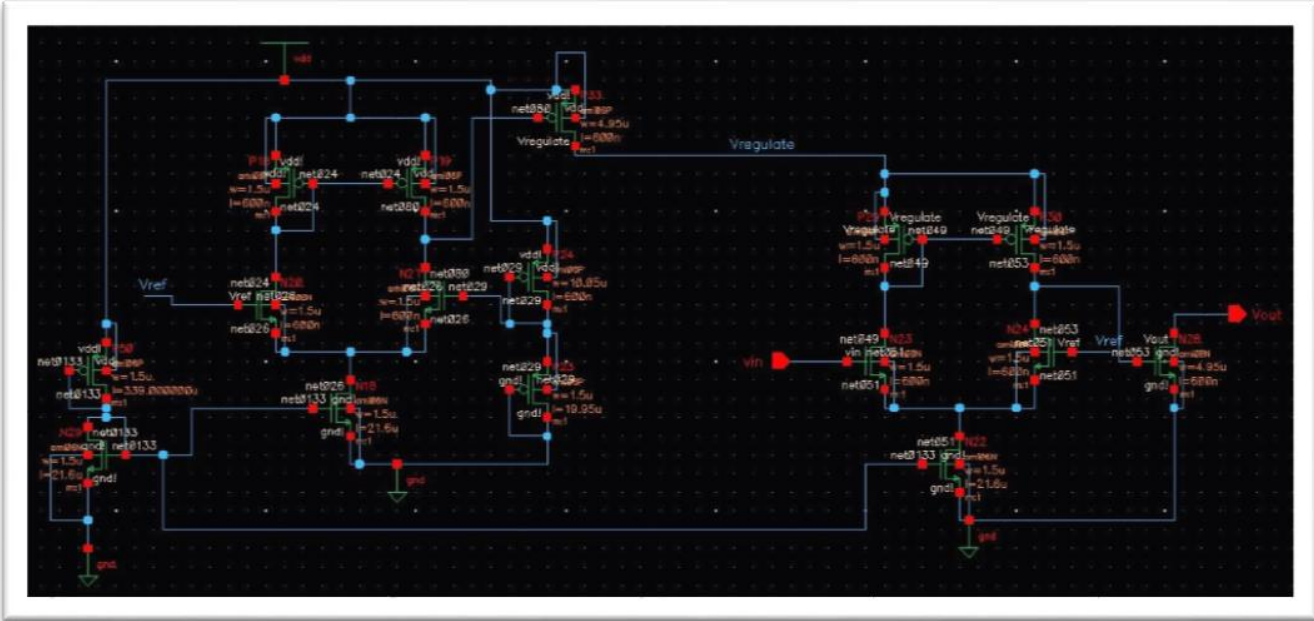

Figure 2.11 Low Voltage Cutoff Schematic

Similar to the voltage regulator design, the resistive divider was replaced by MOSFETs that simulate a high resistance value in order to save space.

The final design consisted of a circuit platform embedded in PDMS. The base of the lens consisted of a printed antenna upon a PET substrate. The printed and insulated electro chromic ink lays on the same layer as the PET. There are additional printed insulation layers on top of the antenna upon which the battery and IC are bonded using a thermo-epoxy. The battery, IC, and electro chromic are connected via wire-bonding (see Chapter 6.3), which can be seen in Figure 2.12. The entire system is embedded in PDMS. 


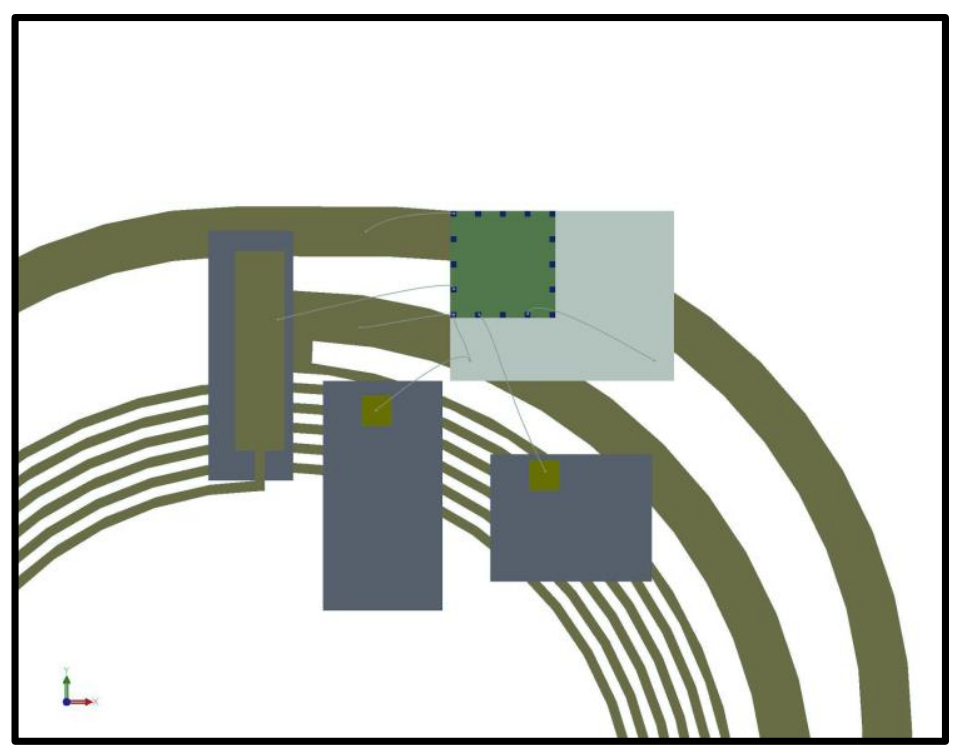

Figure 2.12 The wiring schematic of interconnections between device components Our modular focus for the analysis and testing of device components resulted in a well-defined set of geometries and component characteristics that integrated to form our final device. IC simulations using Cadence Virtuoso showed a total current draw of $5.41 \mathrm{uA}$. Inductive charging required $4.5 \mathrm{Vp}$ at the input of the chip. The display will activate when the IC sees $1 \mathrm{dBm}$ from the wireless sensor. Based upon the desired material characteristics and size constraints of the antenna and IC, the geometry of the lens was designed to match the curvature of the eye as closely as possible. The edge-to-edge diameter of the lens was $1.5 \mathrm{~cm}$, with a thickness of 950 ums. This allowed for a 50 um layer of PDMS on the top and bottom of the lens with 200um of edge coverage. 


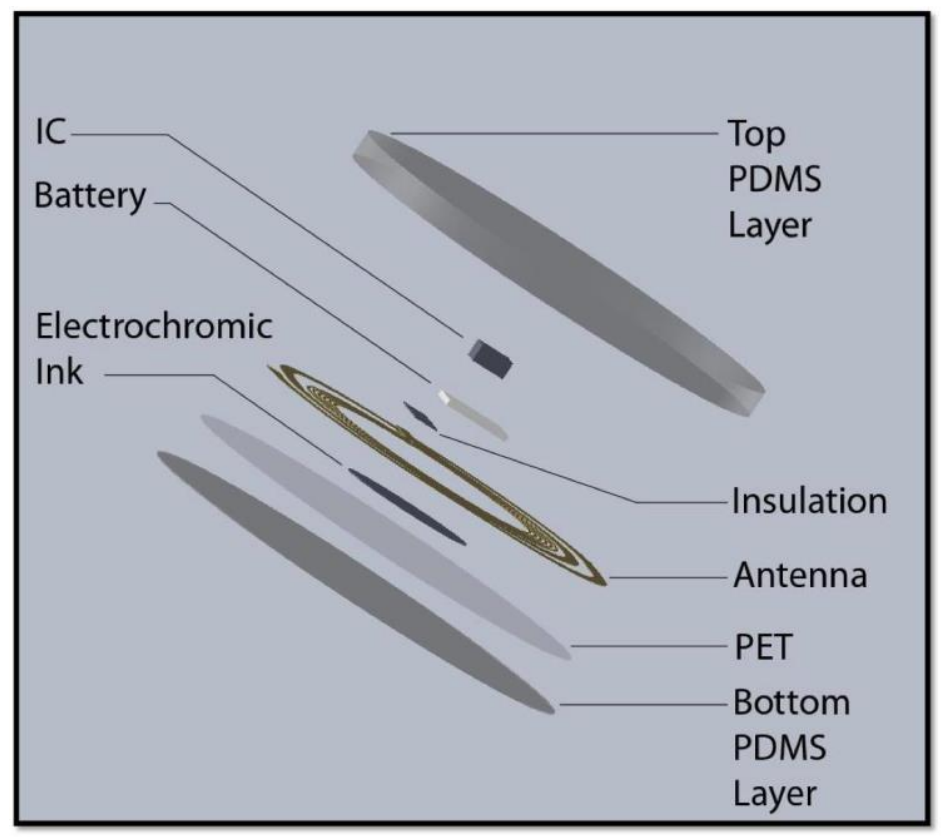

Figure 2.13 Exploded view of lens highlighting individual layers and components Usable area for circuitry is very limited for a contact lens. Having components placed side by side may cause areas that overlap with the pupil, especially with the utilization of the thin film battery. Therefore, the approach taken in this thesis was to stack components on top of each other. This strategy increases of the overall thickness of the lens which compromises the comfort of the contact lens. In addition, a contact lens that is too thick may shift around when the user blinks. However, a thicker contact lens may still be more comfortable as they can fit the eye's curvature better than one that has components laid side-by-side. Figure 2.14 shows the overall system of the active contact lens. 


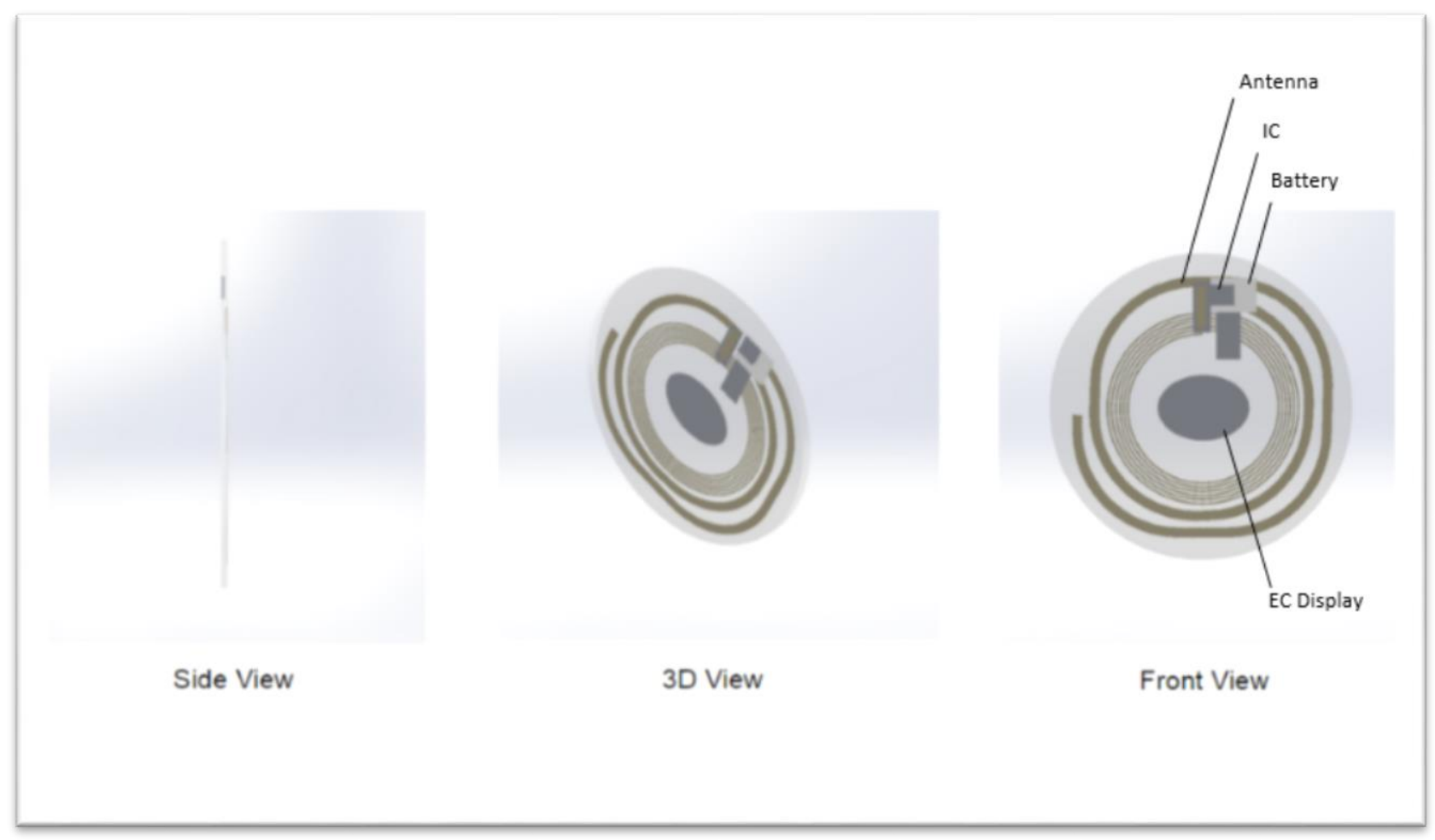

Figure 2.14 Different Views of the Entire Contact Lens

The subsequent chapters of this thesis will discuss the components that are interfaced with the IC which are the antenna, power sources, and display. It will also compare and contrast the two different processing techniques to determine overall which is more suitable process. 


\section{Chapter 3}

\subsection{Antenna Fabrication and Testing}

The primary function of the contact lens is to warn the wearer when a signal caused by an event is received from an external or internal sensor. In order to support its functionality, the contact lens must be able to: receive incoming signals from the sensor, manage the battery's power, and activate a display as a warning stimulus. Reception of the wireless signal and inductive charging is handled by a planarized hybrid antenna. For this device, there are two modes of operation. The first mode acts as a receiving antenna for a $2.4 \mathrm{GHz}$ wireless signal and the second mode performs as the tag coil for a $13.56 \mathrm{MHz}$ charging voltage. A $2.4 \mathrm{GHz}$ operating frequency was chosen because of its small wavelength, thus resulting in a smaller antenna. There was consideration in operating at a higher frequency of $5 \mathrm{GHz}$, which would decrease the size of the antenna even more. Unfortunately, when the operating frequency is increased, the distance of receiving a communication signal decreases. This shortened operating range is due to higher attenuations when the higher frequency

propagates in free space. Also, communicating at $5 \mathrm{GHz}$ is more challenging compared to $2.4 \mathrm{GHz}$ because its frequency band has higher noise. The size of the antenna was limited to a minimum inner diameter of $6 \mathrm{~mm}$ to avoid overlapping the pupil and a maximum diameter of $13 \mathrm{~mm}$ to stay within actual scale size of a contact lens. Screen printing and microfabrication were selected as fabrication techniques and compared to see which had better results for manufacturing an antenna on a flexible, transparent, and thin substrate. 


\subsection{Background for Antenna Design}

The antenna and battery charging coil were combined into a single device such that the coil will be inductive at our charging frequency of $13.54 \mathrm{MHz}$ and will be resonant at 2.4GHz. The magnetic field generated by a circular loop is given by [7]

$$
B_{z}=\frac{\mu_{o} I N a^{2}}{2\left(a^{2}+r^{2}\right)^{3 / 2}}
$$

where $\mu_{0}$ is the permeability of free space, $I$ is the current, $N$ is the number of turns, $a$ is the radius of the loop, and $r$ is the distance from the center of the loop. Figure 3.1 below illustrates these parameters.

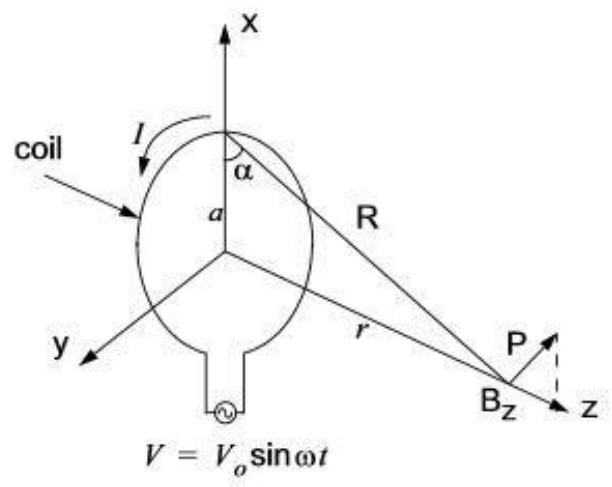

Figure 3.1 Magnetic Field Generated by Single Loop

Equation 3.1 states that the magnetic field generated by the coil is increased by either having more turns or increasing loop area. We must also consider the efficiency of the antenna when receiving a $2.4 \mathrm{GHz}$ signal which is determined by its radiation resistance and Ohmic resistance.

The radiation resistance $\mathrm{Rr}$ of an electrically small antenna is [8]

$$
R_{r}=20\left(\beta^{2} N S\right)^{2} \approx 31,200\left(\frac{N S}{\lambda^{2}}\right)^{2} \Omega
$$


where $\mathrm{N}$ is the number of turns, $\mathrm{S}$ is the loop area, and $\lambda$ is the wavelength. The ohmic resistance of a loop constructed with wire s given by [8]

$$
R_{w}=N^{2} \frac{2 \pi b}{w t} R_{S}
$$

where $b$ is the mean loop radius, $w$ is the trace width, and $t$ is the ink film thickness. Rs is the surface resistance given by

$$
R_{S}=\sqrt{\frac{\omega \mu}{2 \sigma}}
$$

where $\sigma$ is the conductivity of the silver conductive paste. The antenna efficiency is given by

$$
e_{r}=\frac{R_{r}}{R_{A}}
$$

where $R_{A}$ is the sum of $R_{W}$ and $R_{r}$. Having a large radiation resistance will improve the efficiency of our antenna. Therefore, increasing inductance by having a larger inner diameter is a better choice than having more turns because a larger diameter increase the area of the loop and consequently its radiation resistance. The main focus was to optimize the dimensions of the coil such that it has a purely real impedance of $50 \Omega$ (input impedance of the chip) as we would like to avoid using lumped passive components for impedance matching. The efficiency of the antenna was expected to be very low because of its inherent design. First, the loop encompasses a small area which results in a low radiation resistance. Second, the silver conductive paste used for the traces has a high resistivity of $2.33 \times 10^{6} \mathrm{~S} / \mathrm{m}$ which is approximately 18 times less conductive than gold at $4.1 \times 10^{7} \mathrm{~S} / \mathrm{m}$.[8] This resulted in high ohmic losses. Antenna parameters such as trace width and number of turns were determined using simulations 
done on Agilent's Advance Design System (ADS). The table below shows the antenna design optimized for size, efficiency, and $\mathrm{S} 11$ reflection coefficient.

\begin{tabular}{|c|c|}
\hline Outer Diameter $(\mathbf{c m})$ & 1.3 \\
\hline Inner Diameter $(\mathbf{m m})$ & 8 \\
\hline Trace width $(\boldsymbol{\mu m})$ & 250 \\
\hline Trace thickness $(\boldsymbol{\mu m})$ & 250 \\
\hline Number of turns & 4 \\
\hline
\end{tabular}

Table 1 Printed Antenna Parameters

The antenna was created using the screen printing and microfabrication

process. The substrate in the printing process is a $25 \mu \mathrm{m}$ thick PET film as PET

is a standard in screen printing. However, PET is an irritant and a more

biocompatible substrate would be better suited for use in the eye. The substrate chosen in the microfabrication process was PDMS based on its known biocompatibility as a contact lens and high oxygen permeability. The ink used to print the trace is 5052 Silver Conductive Paste from DuPont and the material for sputtering was pure gold. Figure 3.2(a) and 3.2(b) show the simulated substrate model. 


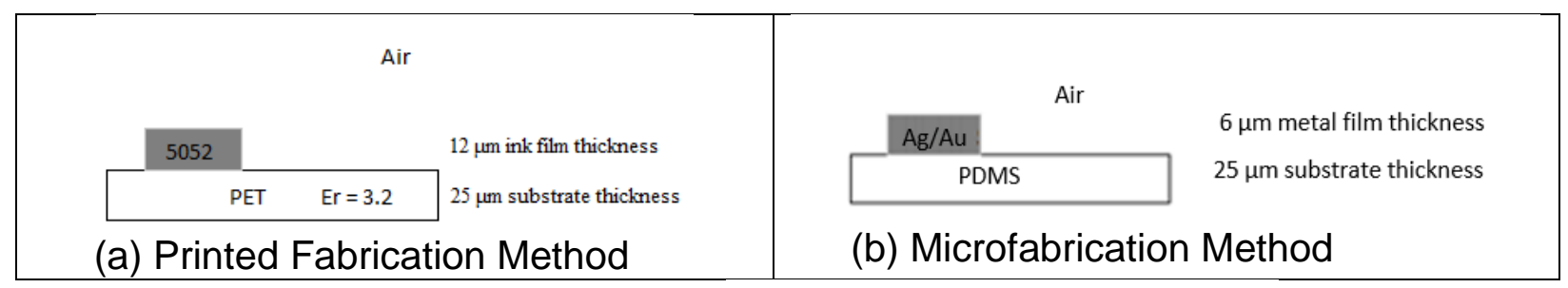

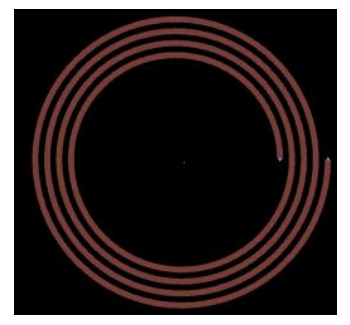

(c) Simulated Coil

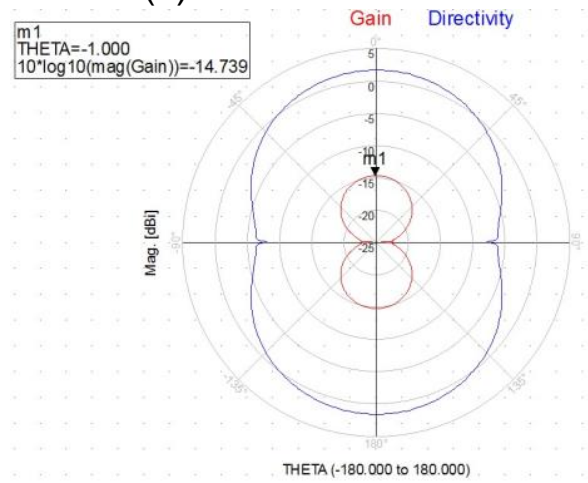

(e) Efficiency of Coil

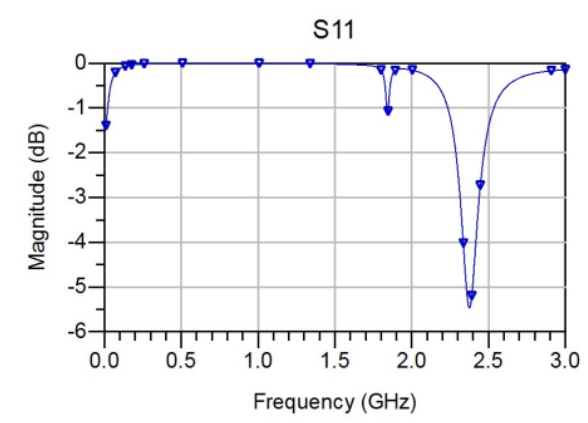

(d) S11 of Coil

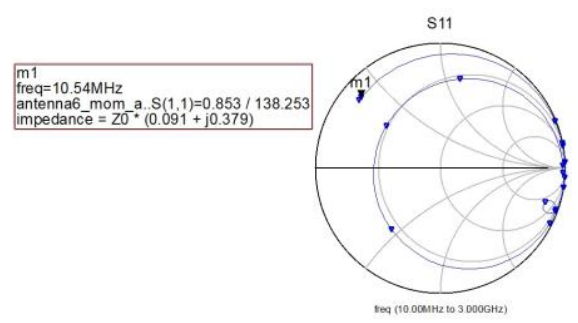

(f) Efficiency of Coil

Figure 3.2 Response of Coil Design

Figure 3.2(c) shows the antenna layout in ADS. Figure 3.2(d) shows the simulated reflection coefficient at $2.375 \mathrm{GHz}$. Figure $3.2(\mathrm{e})$ shows the simulated gain of the antenna. Figure 3.2(f) shows a coil inductance of $286.6 \mathrm{nH}$. The antenna design has a gain of $-14.74 \mathrm{~dB}$ because the extra turns needed to produce inductance create a high series resistance. An alternative antenna design with higher gain was needed to achieve detection at a distance of $32 \mathrm{~cm}$. The motivation for the final design of the antenna was the inverted $F$ antenna (IFA). 
An IFA is a hybrid of a slot antenna and dipole antenna that is vertically polarized. The advantage of the IFA for this design is its large ground plane and that its operating frequency is only determined by the length of a single stub which is $N / 4$. Figure 3.3 below shows a $2.4 \mathrm{GHz}$ IFA for signal detection with a $31.25 \mathrm{~mm}$ stub length and a large ground plane.

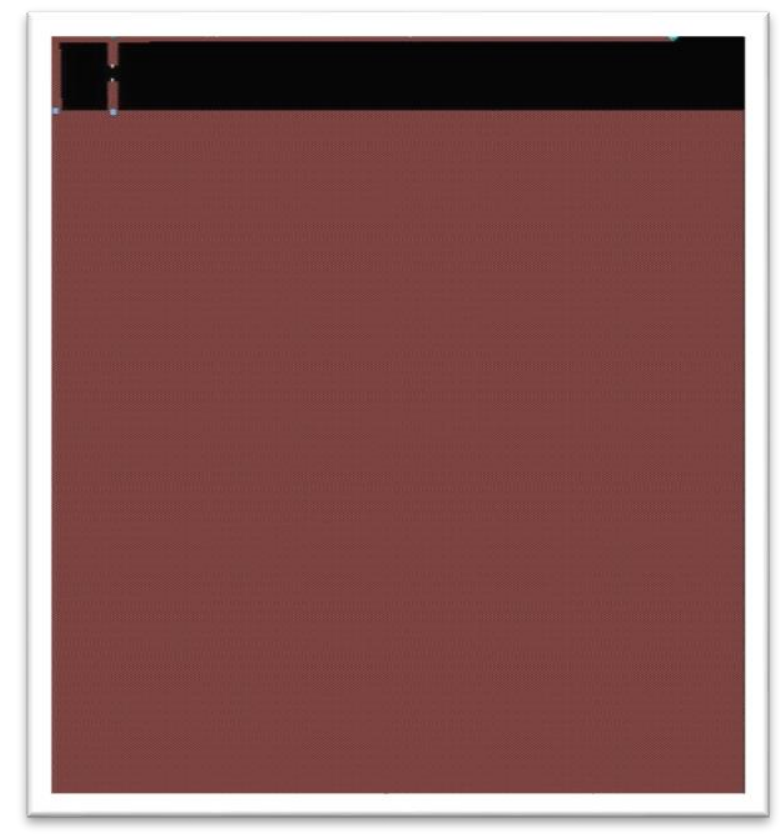

Figure 3.3 Inverted F Antenna

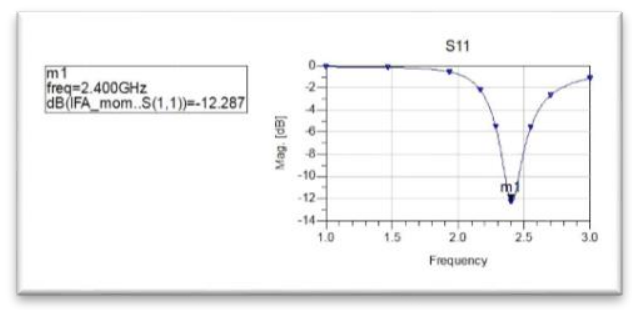

(a) S11 of IFA

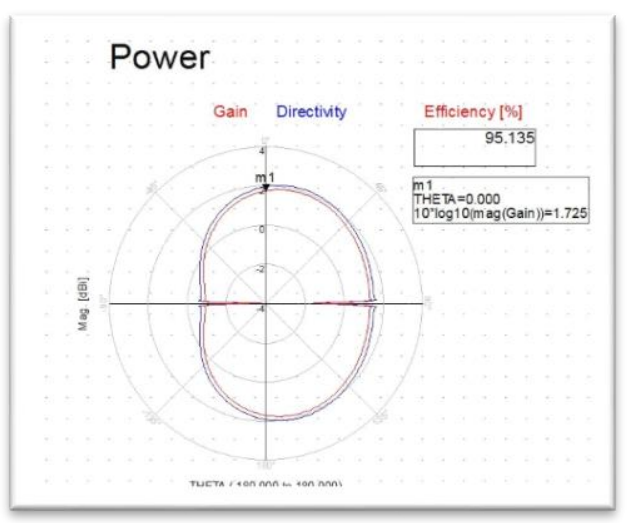

(b) Efficiency of IFA

Figure 3.4 Response of 2.4GHz IFA 
Figure 3.4(a) confirms the proper $2.4 \mathrm{GHz}$ operation of the IFA with a reflection coefficient $|\mathrm{S} 11|$ of $-12.287 \mathrm{~dB}$. The large ground plane allows the IFA to achieve an efficiency of $95 \%$. Ideally, the height of the ground plane and the length of the stub should have a dimension of $31.25 \mathrm{~mm}$ which is a quarter-wavelength for $2.4 \mathrm{GHz}$. The size of the IFA's ground plane does not affect the resonant frequency of the antenna. Therefore, the ground plane was altered to fit the viewing parameters of the user by cutting a hole in it.

Unfortunately, lower efficiencies and smaller bandwidths would result in ground plane distortion. In order to fit the IFA into a contact lens, its shape was adjusted such that it was circular and would allow the eye to see through. The first requirement was achieved by changing the ground plane to be circular instead of a square. The stub was then curved and wrapped around the ground plane while keeping the same length. To address the visibility issue, a hole was created in the ground plane resulting in the antenna structure shown below in Figure 3.5. 


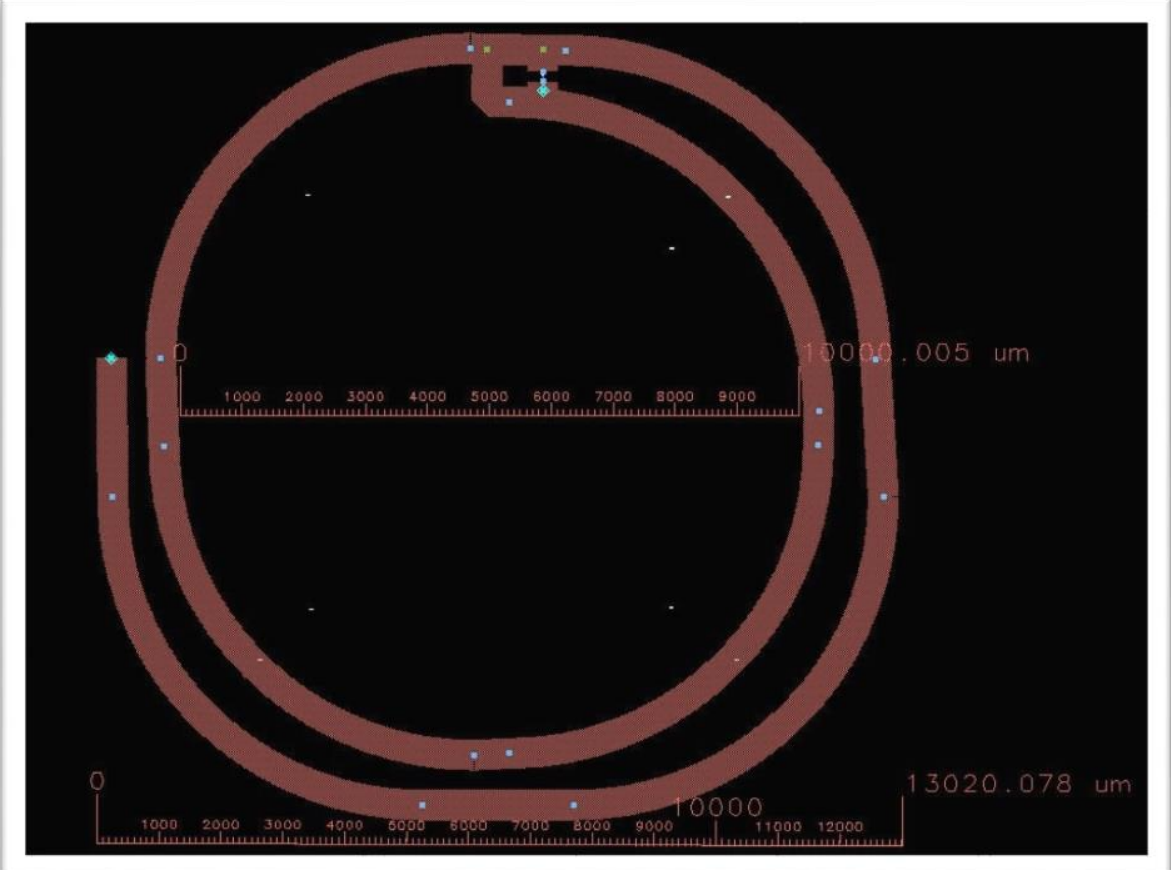

Figure 3.5 Curved IFA with Opened Ground Plane (top view)

The IFA was curved to fit within the constraints of a $1.3 \mathrm{~cm}$ maximum outer diameter and not violate a minimum inner diameter of $8 \mathrm{~mm}$ for the iris.

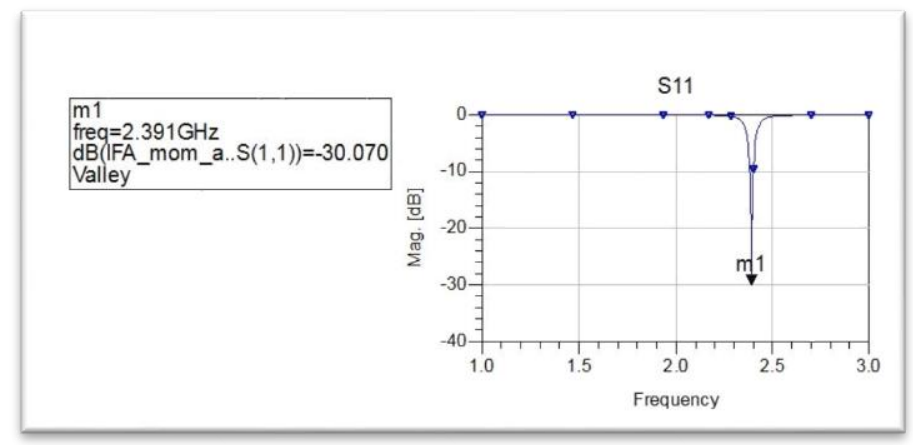

Figure 3.6 S11 of Curved IFA Design 


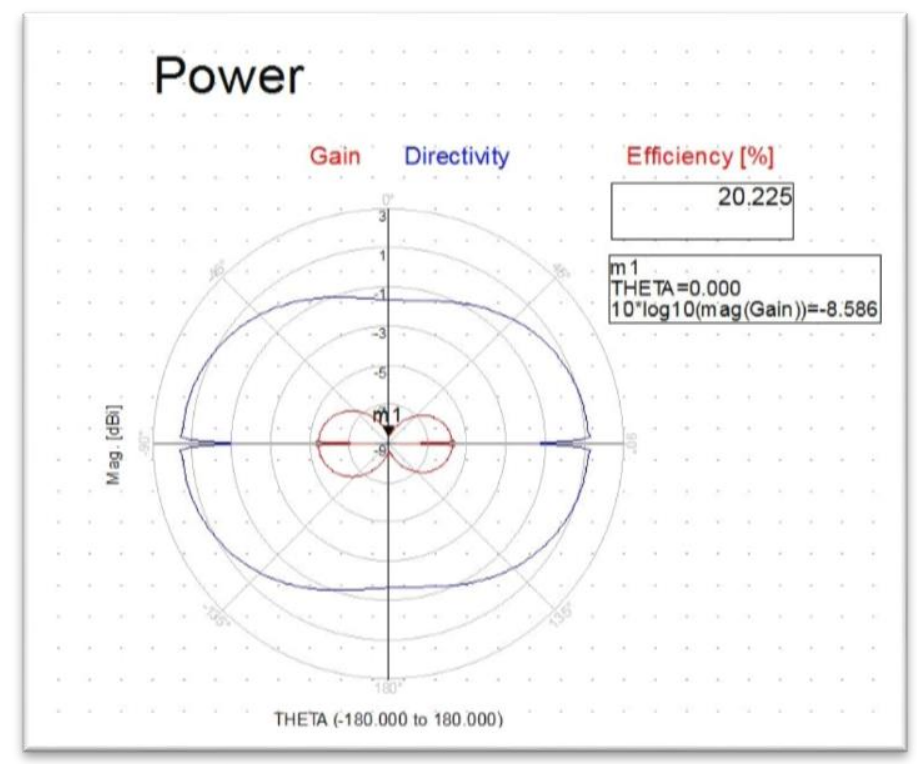

Figure 3.7 Efficiency of Curved IFA Design

Figure 3.6 shows a minor shift of $9 \mathrm{MHz}$ lower in the resonant frequency of the antenna and a lower bandwidth while the efficiency of the antenna drastically dropped from $95 \%$ to $20 \%$ as shown in Figure 3.7. These changes the antenna's characteristics were caused by the change from a ground plane to a ground loop [9]. The lowered bandwidth was due to the added parasitic capacitance and inductance of the loop with the curved stub and the lowered efficiency created by the smaller ground plane causing higher ohmic losses. By opening a large circular area inside the ground plane of the IFA, a planarized coil was attached for inductive charging application to the lens at a frequency of $13.56 \mathrm{MHz}$.

Inductive charging is accomplished by applying a time-varying current through a coil. The $A C$ current generates a time-varying magnetic field that induces an $A C$ voltage at the receiving coil. The amplitude of the induced voltage at the receiving coil is proportional to the magnetic field created by the transmitting coil. This means a large 
magnetic field is desired on the transmission side and was calculated using the following equation [7]:

$$
B_{Z}=\frac{\mu_{0} I N a^{2}}{2\left(a^{2}+r^{2}\right)^{3 / 2}}
$$

where $\mu_{0}$ is the permeability of free space, $I$ is the current, $N$ is the number of turns, $a$ is the radius of the loop, and $r$ is the distance from the center of the loop. Equation 3.6 states that the magnetic field generated by the coil is increased by either having more turns, or increasing the loop area. The inner diameter of the coil is limited by the diameter of the pupil, which in low light conditions ranges around $7 \mathrm{~mm}$. The design was based on fabrication limitation where the smallest feature size achievable through microfabrication is $5 \mu \mathrm{m}$ trace widths. However, this is not the case for the screen printing process on campus which is $100 \mu \mathrm{m}$ trace widths as the mesh sizes of the screens are too large to achieve thinner traces. Additionally, to tailor toward both processing sides, the outer diameter of the coil cannot completely occupy the $10 \mathrm{~mm}$ diameter that is available inside the ground loop. The reason for this is the small gap between the ground loop and the coil could cause shorts in the printing process. Taking these limitations into consideration, the designed coil had an inner diameter of $7 \mathrm{~mm}$ and an outer diameter of $9.6 \mathrm{~mm}$. This allowed a maximum number of 6 turns with $100 \mu \mathrm{m}$ trace widths and spacing which can be seen in the final design shown in Figure 3.8 below. 


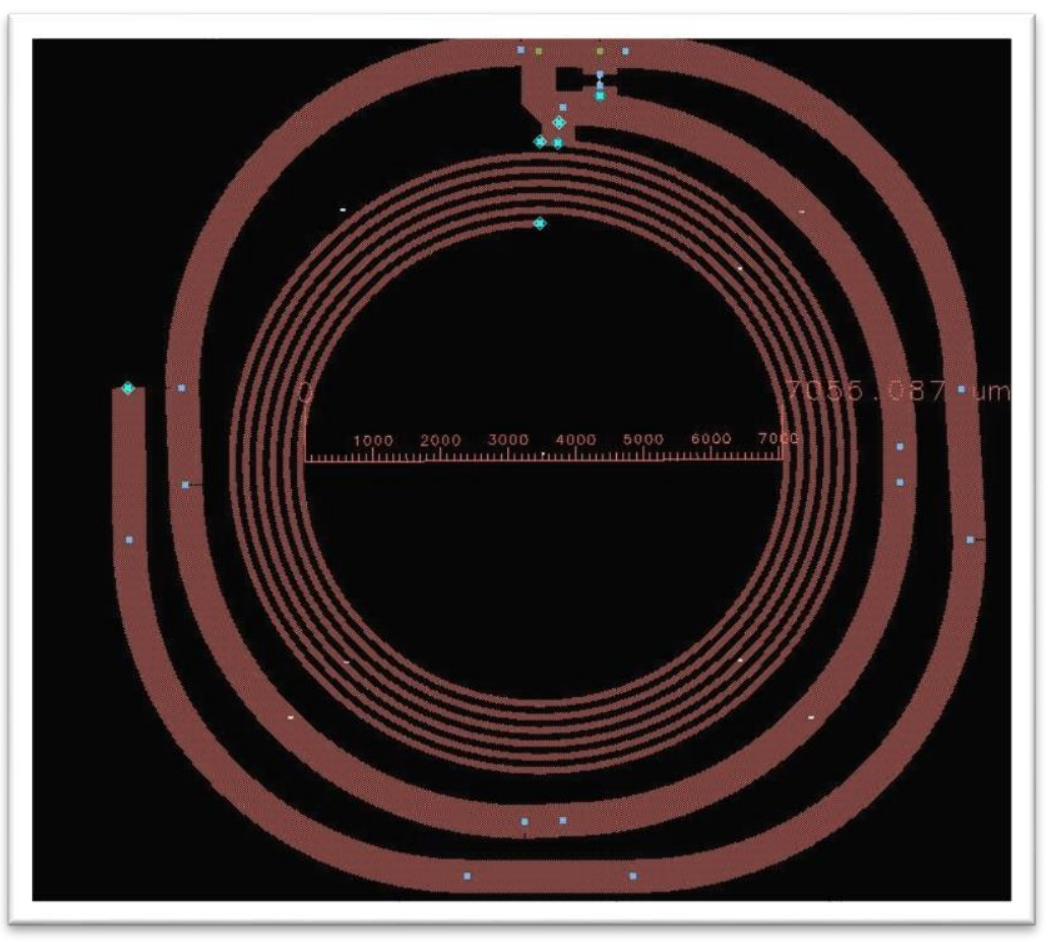

Figure 3.8 Final Antenna Design

Figure 3.9 shows the impedance of the inductive coil. The coil was simulated using a 15 $\Omega / \mathrm{sq} / \mathrm{mil}$ sheet resistivity in order for the conductor to account for the resistance of the conductive ink used for screen printing.

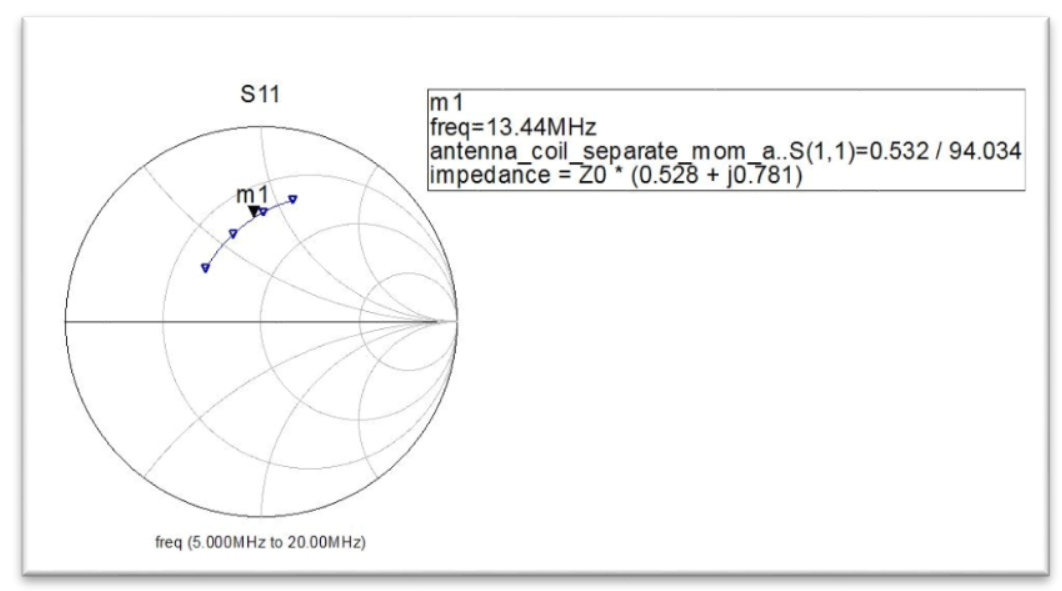

Figure 3.9 Smith Chart of Coil 
The simulations produced a resistance value of $26.4 \Omega$ and inductance value of $0.46 \mu \mathrm{H}$ in a $50 \Omega$ system giving a $Q$ factor of 1.479 . If a $10 \mathrm{Vp}$ sinusoid is applied to the coil, the resulting $100 \mathrm{mAp} A C$ current produces a flux density of $107.66 \mu \mathrm{Wb} / \mathrm{m}^{2}$ when calculated using equation 3.7. The receiving coil's induced voltage is calculated using [7]:

$$
V_{0}=2 \pi f N S Q B_{0} \cos (\alpha)
$$

where $f$ is the $13.56 \mathrm{MHz}$ operating frequency, $S$ is the area of the coil, $Q$ is the $Q$-factor, $B_{0}$ is the arriving magnetic field, and $\alpha$ is an arrival angle of $90^{\circ}$. The calculations show that a $10 \mathrm{Vp} 13.56 \mathrm{MHz}$ sinusoid wave from the transmitting coil should produce a peak voltage of $3.11 \mathrm{~V}$ at the receiving coil when the two coils are placed directly on top of each other. Unfortunately, when the coil is added to the IFA it decreased the gain by $0.8 \mathrm{~dB}$ which lowered the efficiency by $3 \%$. It was determined that the added coil increased the resistivity of the ground plane resulting in a higher ohmic loss. 


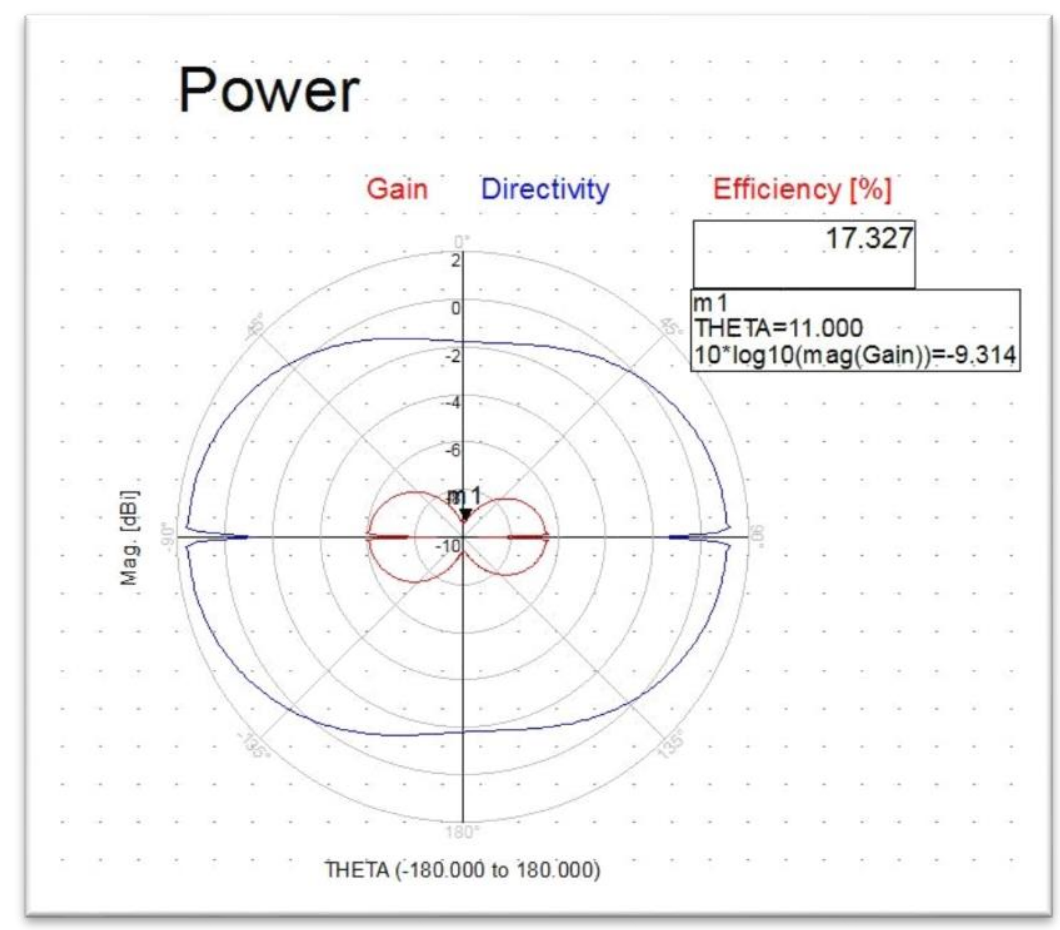

Figure 3.10 Gain of IFA Design

\subsection{Printing Fabrication Process}

While the antenna was designed such that it was small enough to fit on a contact lens, a large portion of this work consisted of determining a suitable fabrication process. With the help of Dr. Malcolm Keif and the Graphic Communications (GRC) department, the technique of screen printing was utilized to fabricate the antenna. Screen printing allows us to rapidly prototype, test, and make necessary revisions all while supporting the use of transparent and flexible substrates. Therefore, both antenna designs could be printed for testing with no extra work required. Screen printing is also an additive process eliminating the need for harmful etchants during fabrication. Screen printing works by having a screen where the design is patterned as gaps allowing ink to be pushed through the screen onto a substrate. 
The first step in screen printing is to develop a mask of the design you want.

The mask, seen below in Figure 3.11, was designed using Adobe Illustrator and printed using the Cyrel Digital Imager, seen below in Figure 3.12.

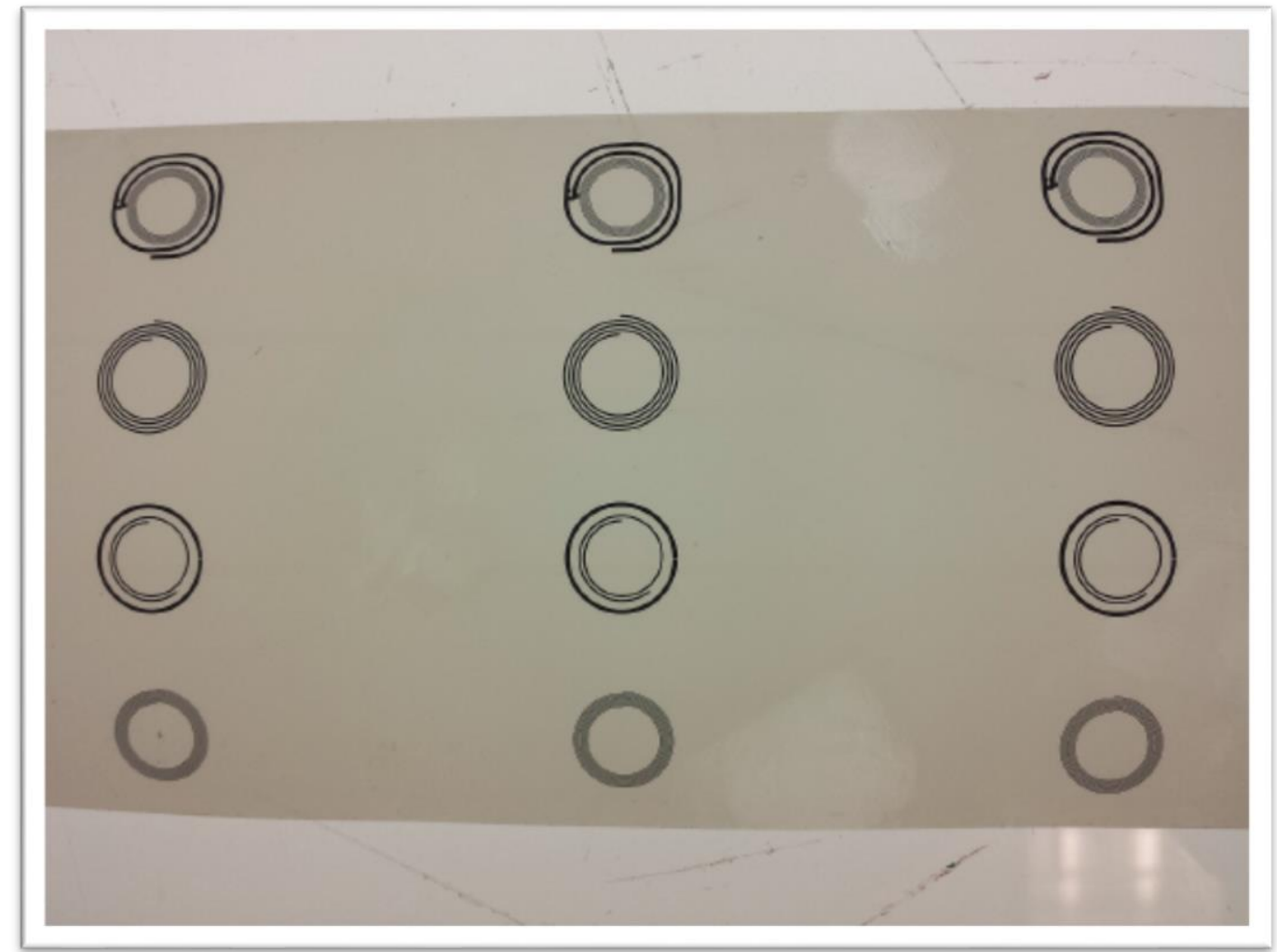

Figure 3.11 Mask for antenna fabrication 


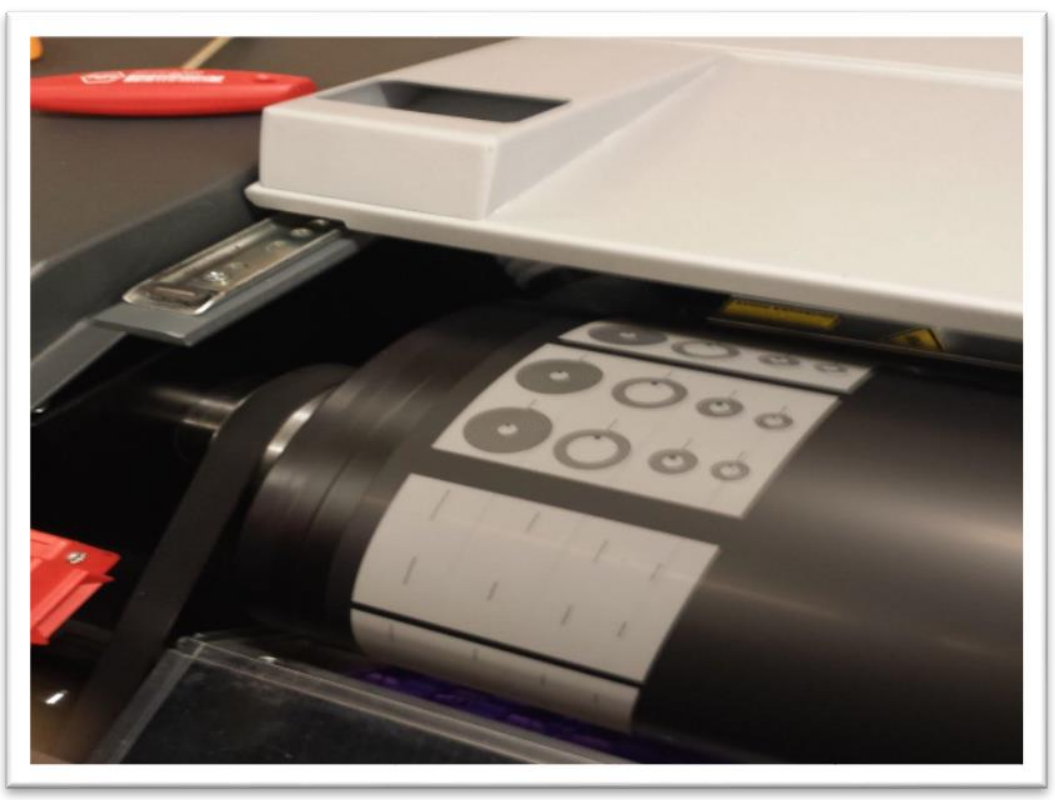

Figure 3.12 Cyrel Digital imaging machine printing antenna mask

The next step required applying an emulsion layer to a 420 lines per linear inch mesh. This is very fine screen size that allows features of up to 100 um to be printed. The emulsion was then cured using UV light. The mask was placed in between the screen and the UV seen in Figure 3.13 below. 


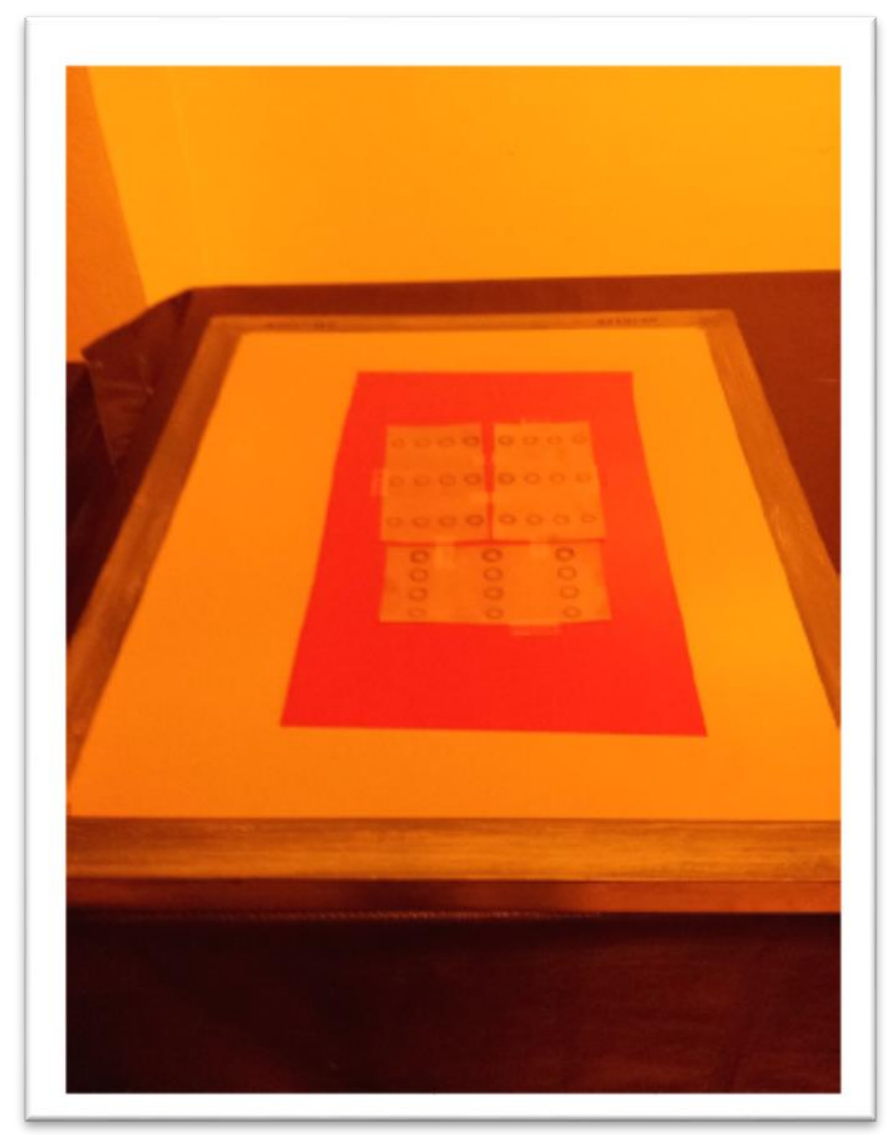

Figure 3.13 Mask Laid On Top of Emulsion

The areas of the mask that block the UV light prevent the emulsion from curing. This allows the unexposed parts to stay water soluble and to be washed away, as seen in Figure 3.14 below. This process must be done in a dark room to prevent UV wavelength light from curing emulsion and preventing it from being removed. If emulsion cures it will remain on the screen and create holes in the final printed antenna design. 


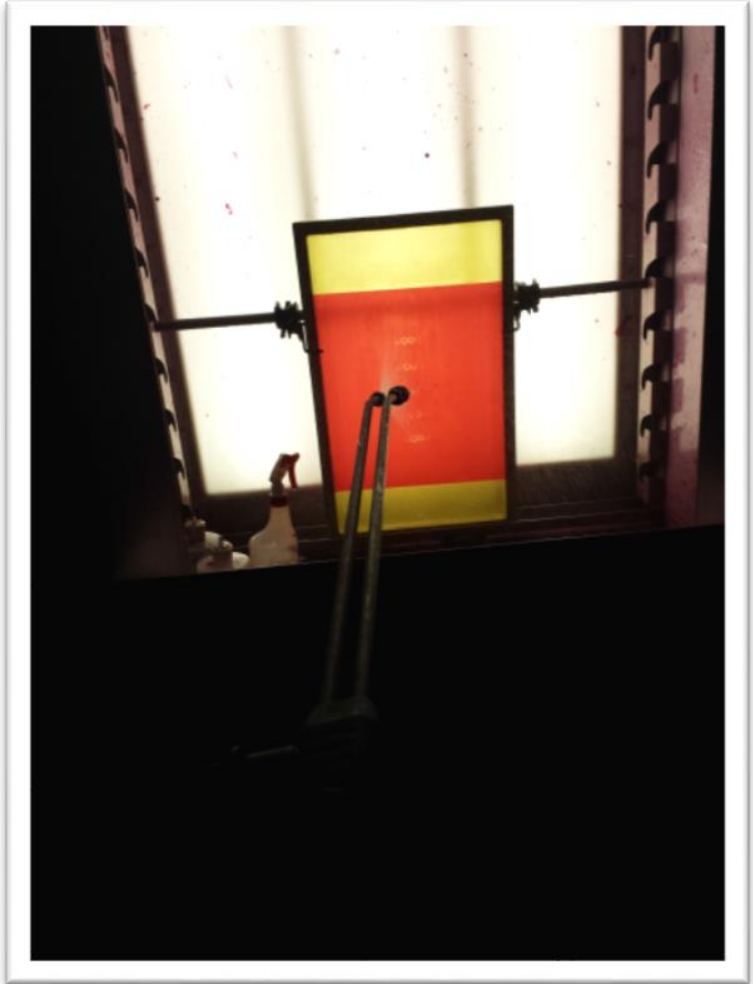

Figure 3.14 Removal of emulsion to create screen features

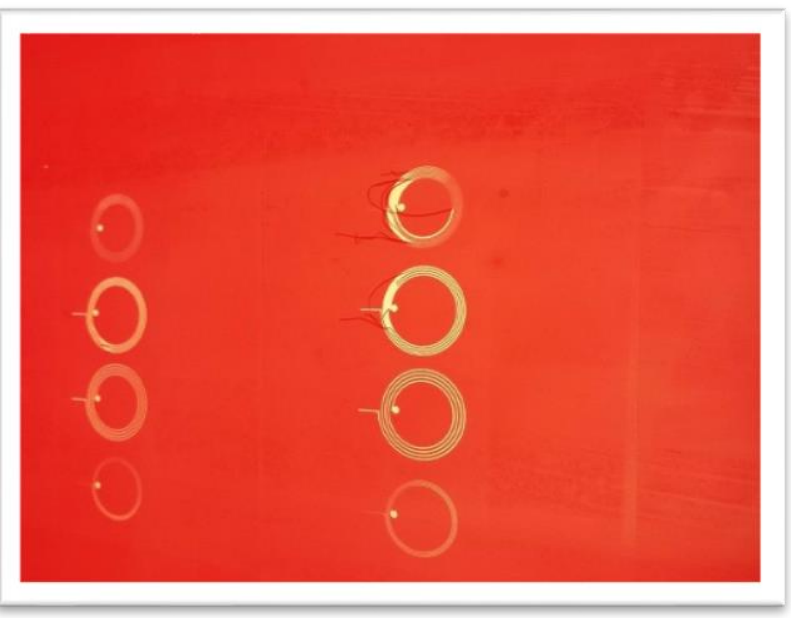

Figure 3.15 Blown Out Design 


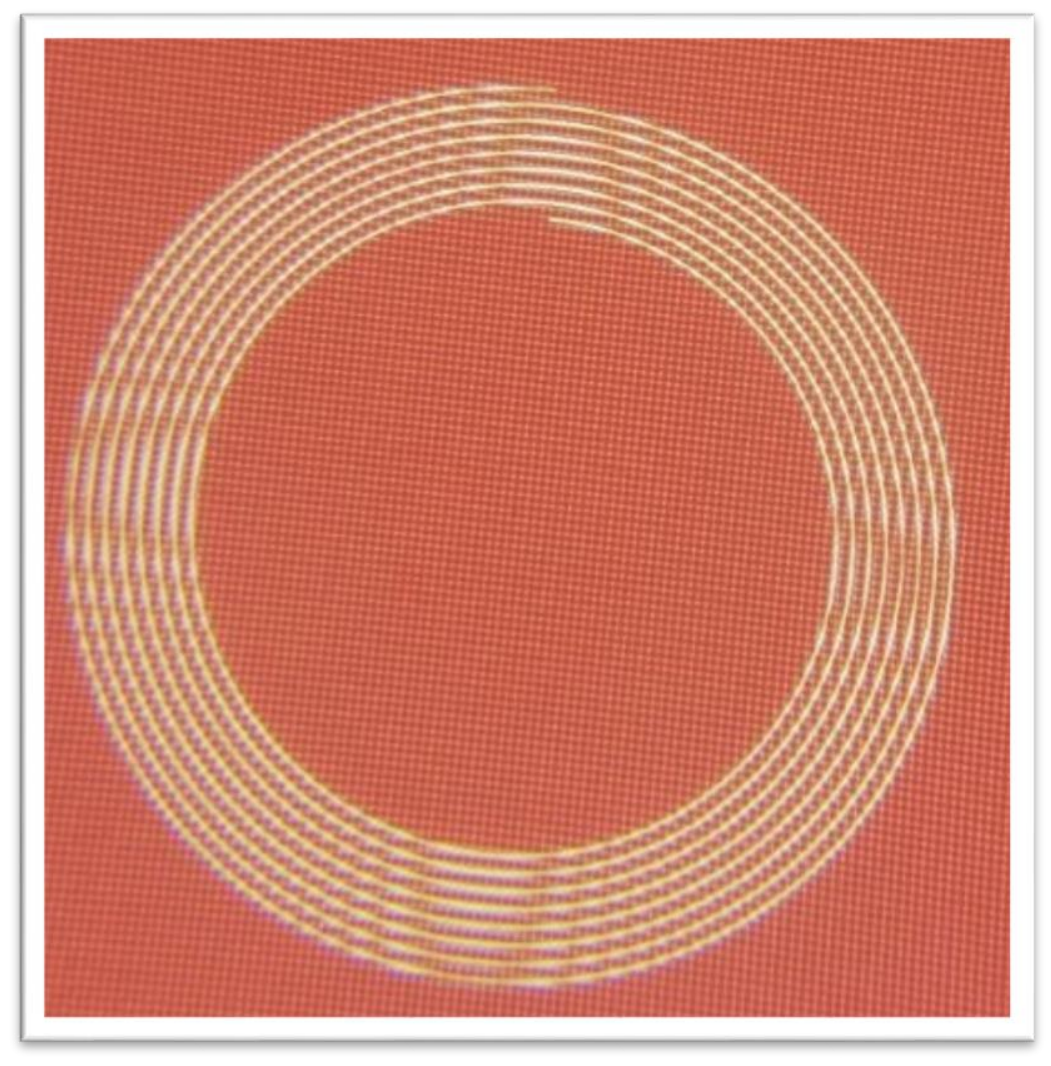

Figure 3.16 Undercutting

In Figure 3.15, a blown out design is shown when the light intensity for curing was set to 90LTU. The areas between traces were not hardened completely and were washed out. Another factor that may also cause a blown out design is improper emulsion adhesion. If the emulsion was not applied with enough force on the screen, the emulsion may not adhere completely and tended to easily wash out. Figure 3.16 shows an example of an overexposure at $140 \mathrm{LTU}$ where the test design was a coil with $100 \mu \mathrm{m}$ trace widths and spacing. Too high of an intensity level will cause the areas underneath the trace in the mask (the black areas of the mask) to become partially exposed. The traces on the screen become thinner than traces on the mask correlating to thinner traces during the print. Figure 3.17 shows the screen that has been exposed correctly using $120 \mathrm{LTU}$. It can be seen that the trace widths were slightly thinner than the spacings indicating 
minor undercutting. However, this was unavoidable as preserving the feature sizes was more important than thinner trace widths. Once the screen has was washed and dried, a second post-exposure at 120LTU was performed to ensure the emulsion was hardened to increase durability.

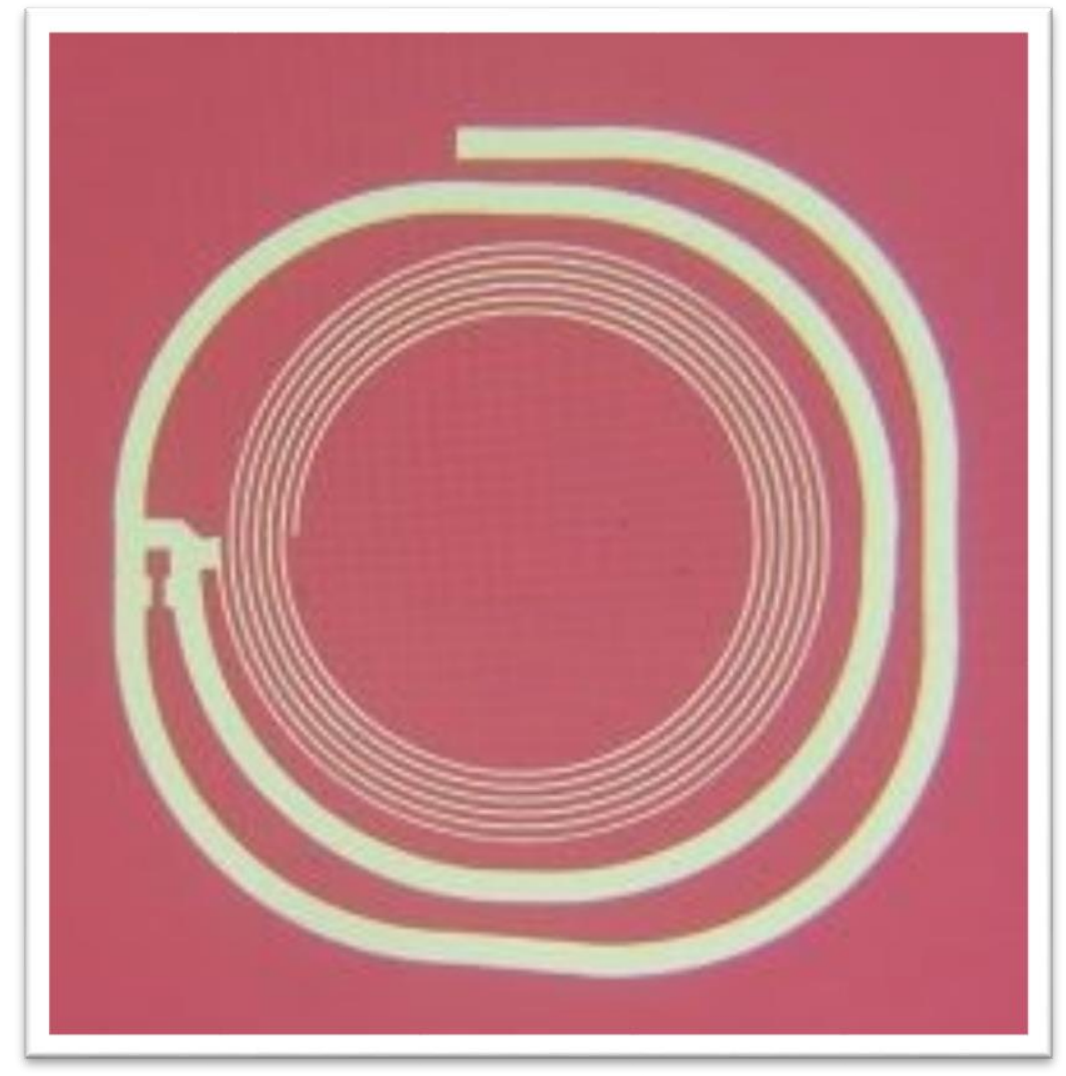

Figure 3.17 Resultant Stencil

In the final step of this process, the PET is placed on the machine, on top of which the screen is placed. The ink is then applied and the automatic applicator is engaged. It is critical to apply the silver ink quickly and engage the applicator, otherwise the ink will dry and ruin the features of the antenna. This process can be seen below in Figure 3.18. The final product can be seen in Figure 3.19 on the next page. 


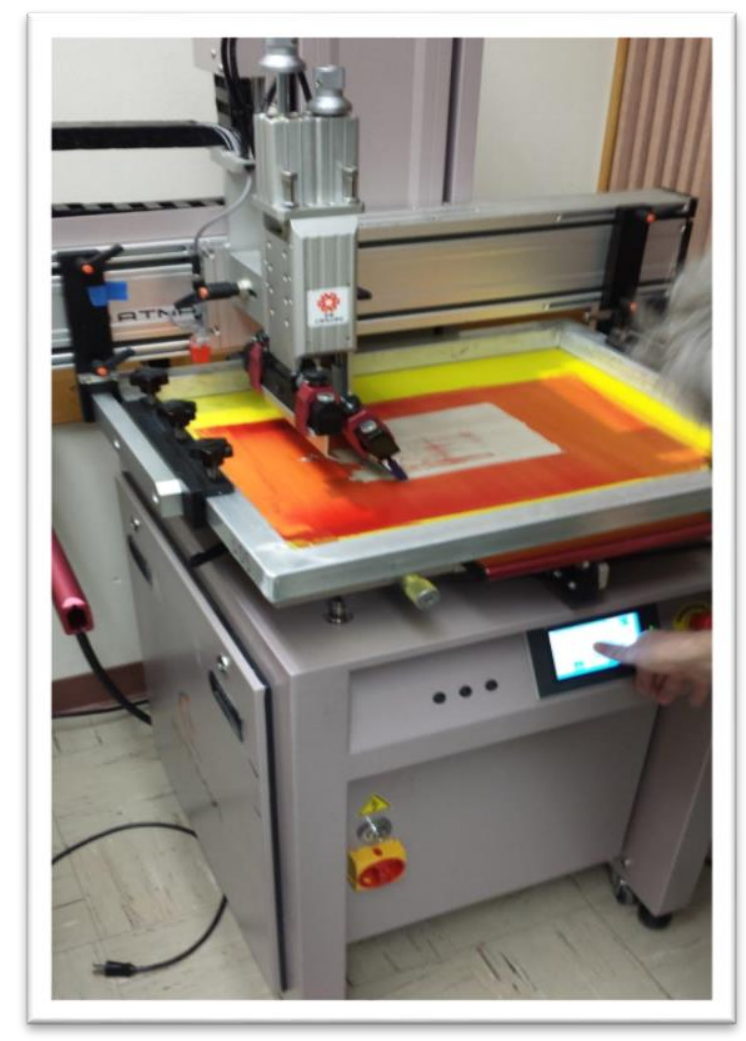

Figure 3.18 Application of the silver ink using the ATMAS machine

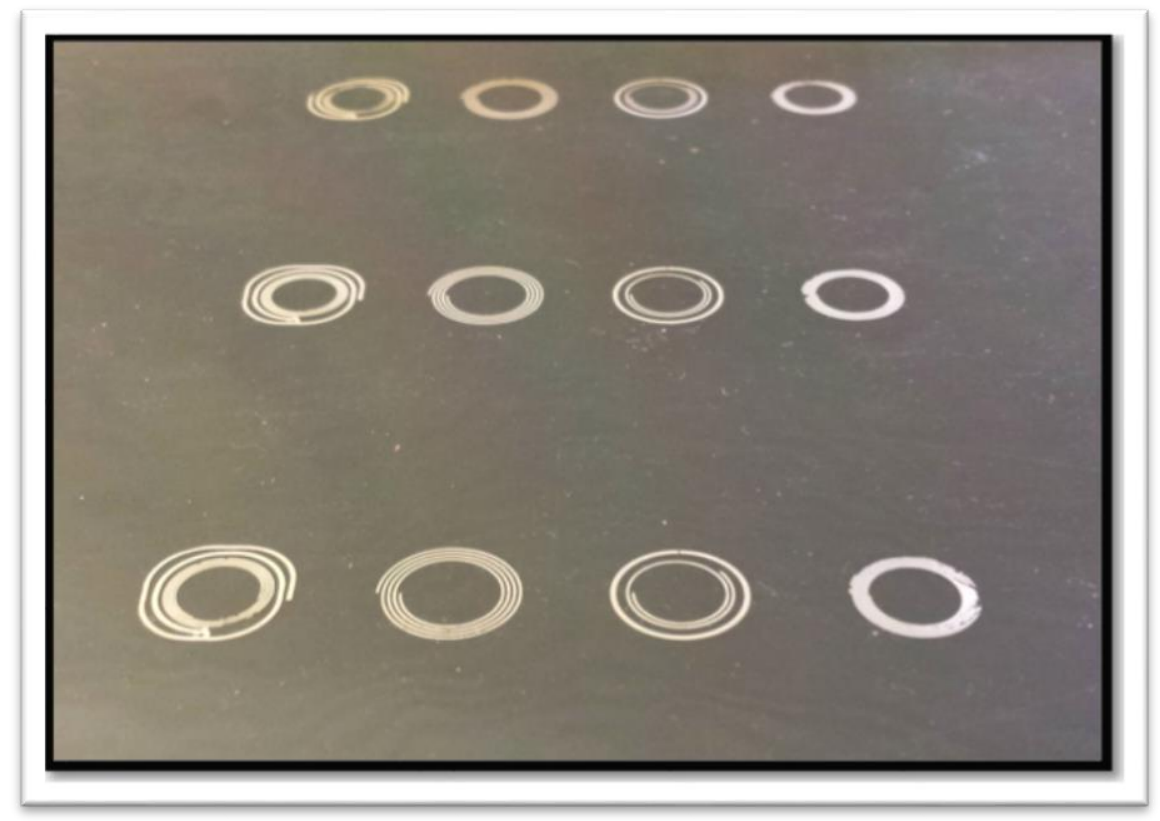

Figure 3.19 Printed antennas on PET substrate 


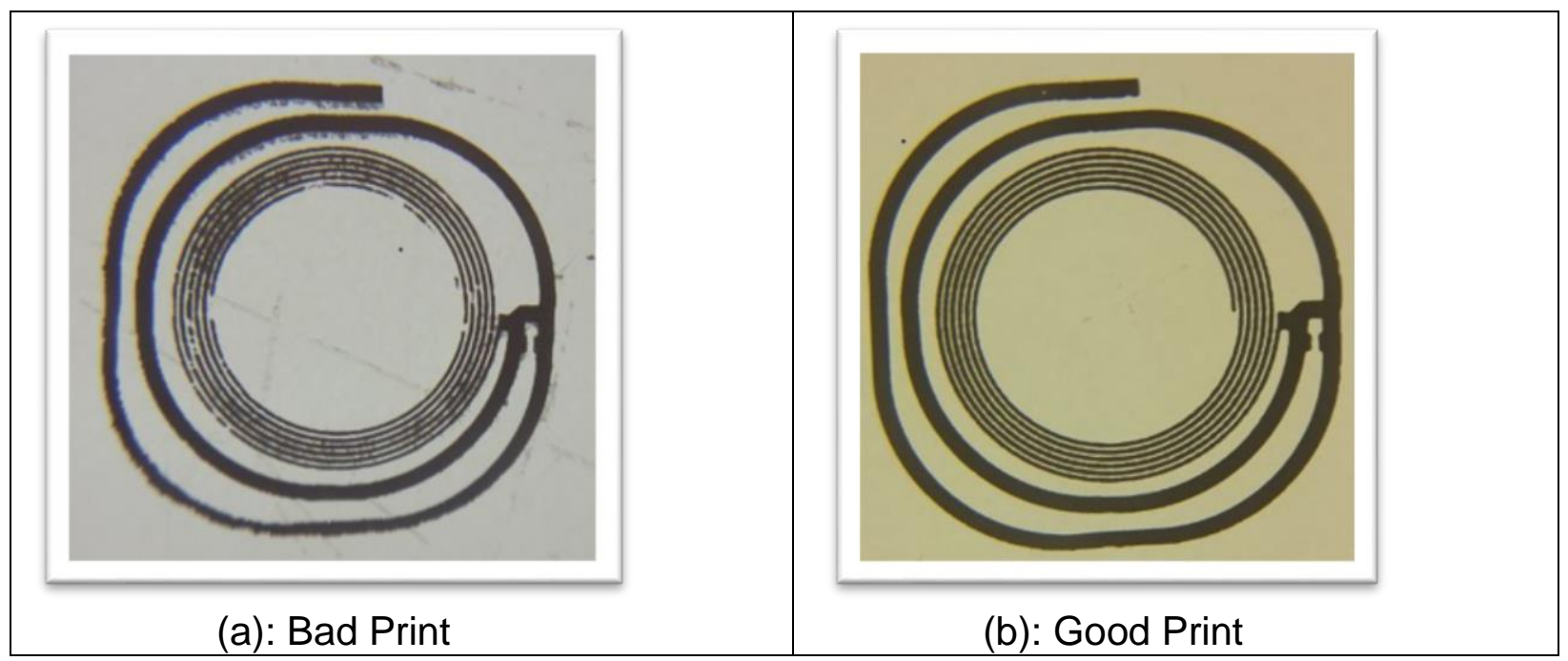

Figure 3.20 Example of Prints

Even though the screen used in Figure 3.18 showed some undercutting, the effects cannot be seen in the final print. A microscopic image was taken to verify continuity and to measure the traces and spacing widths. In Figure 3.21, the traces were thicker than the spacings in between.

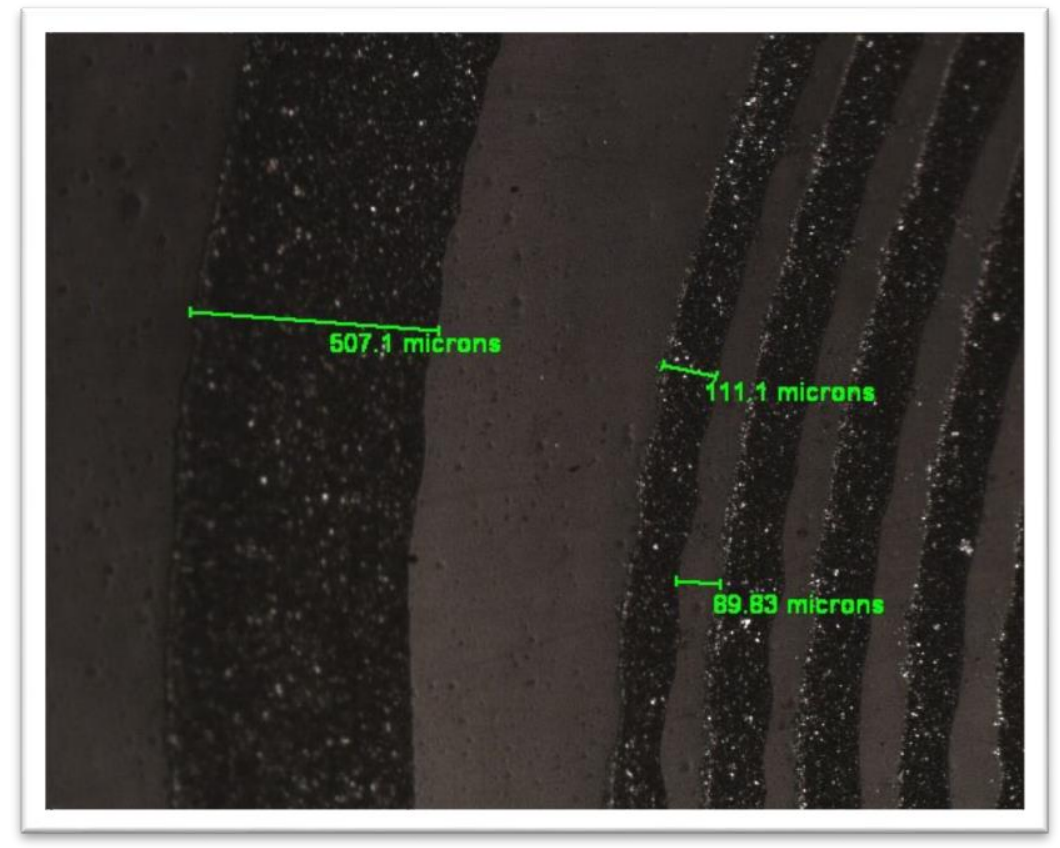

Figure 3.21 Microscope Image of Traces 
The next phase of verification involved determining whether or not the screen printing method of manufacturing the antenna produced a consistent inner diameter free of obstruction. Materials used in this test include calipers accurate to $3 / 1000$ th of an inch, 20 screen printed antennas, and the prototype lens.

Figure 3.22 below shows an example of this test using a ruler.

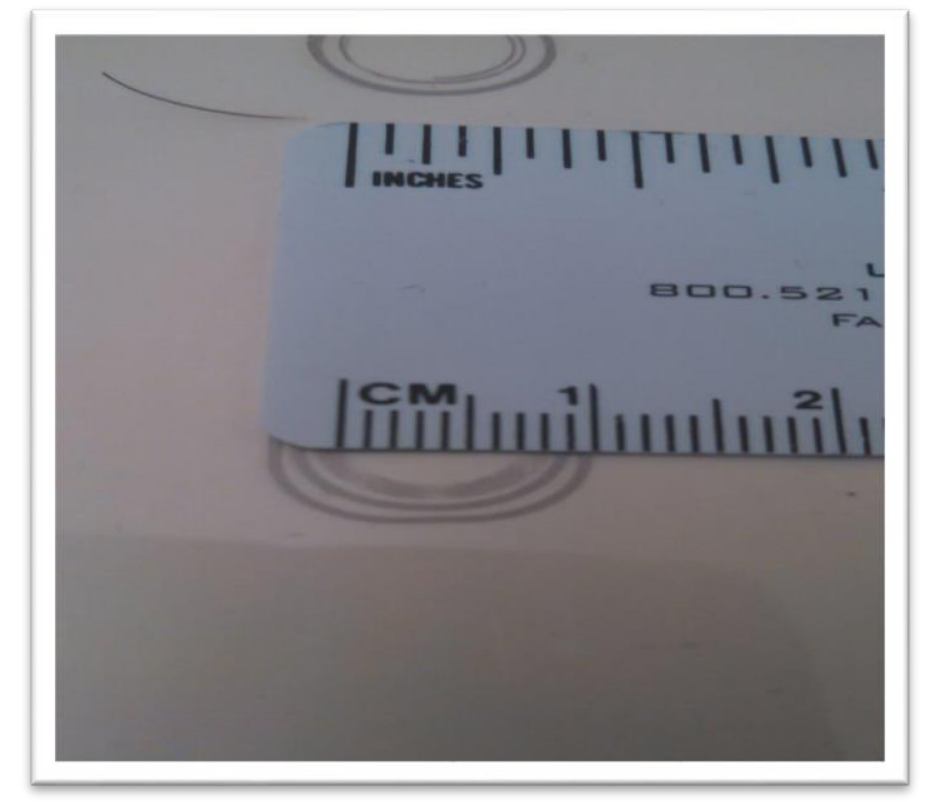

Figure 3.22 Example of antenna printing verification

The result of measuring many antennas including the prototype lens revealed that the printing process is a remarkably accurate manufacturing method. Nearly all antennas were printed with an inner diameter of $8 \mathrm{~mm}$, within the tolerance threshold for the specification. Out of twenty printed antennas, only 2 had defects associated with the arduous process manually screen printing the antennas. These defects caused a slight smearing of ink into the center of the lens. Since the sample size is very small, no statistically powerful conclusion 
can be made regarding the accuracy of the printing process, but for this design, an error rate of $10 \%$ that creates visibly distinguishable defects is very good.

The thickness of the printed traces were also measured. In the case below, the first prototype was printed on $125 \mu \mathrm{m}$ PET substrate and measured with an interferometer. According to the analytical results of the interferometer, the maximum $Z$ distance measured was $142.486 \mu \mathrm{m}$, a substrate thickness of $125.447 \mu \mathrm{m}$, and a trace thickness to be $17.039 \mu \mathrm{m}$. Figure 3.23 below shows the measurement results of the interferometer.
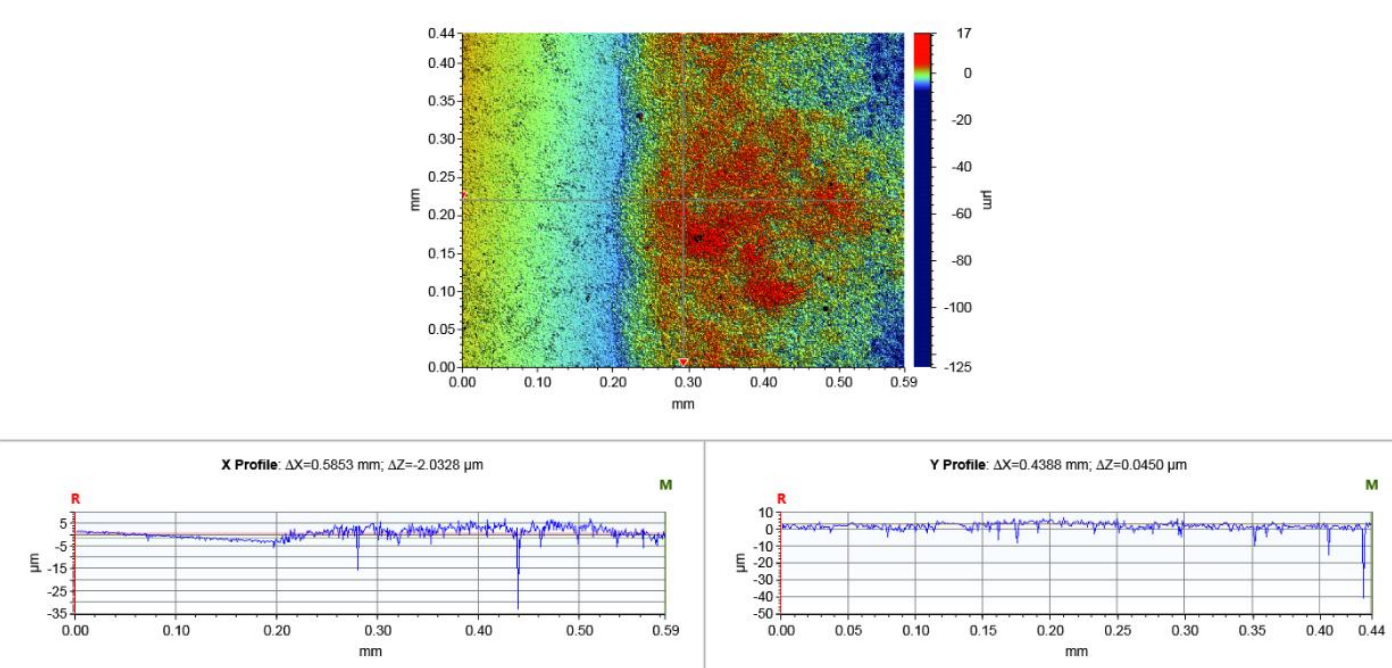

Figure 3.23 Interferometer Data of Printed Trace Sample Later samples were measured with a profilometer in the microfabrication laboratory were the printing height was a consistent height of $12 \mu \mathrm{m}$. Figure 3.24 shows the data acquired by the profilometer. 


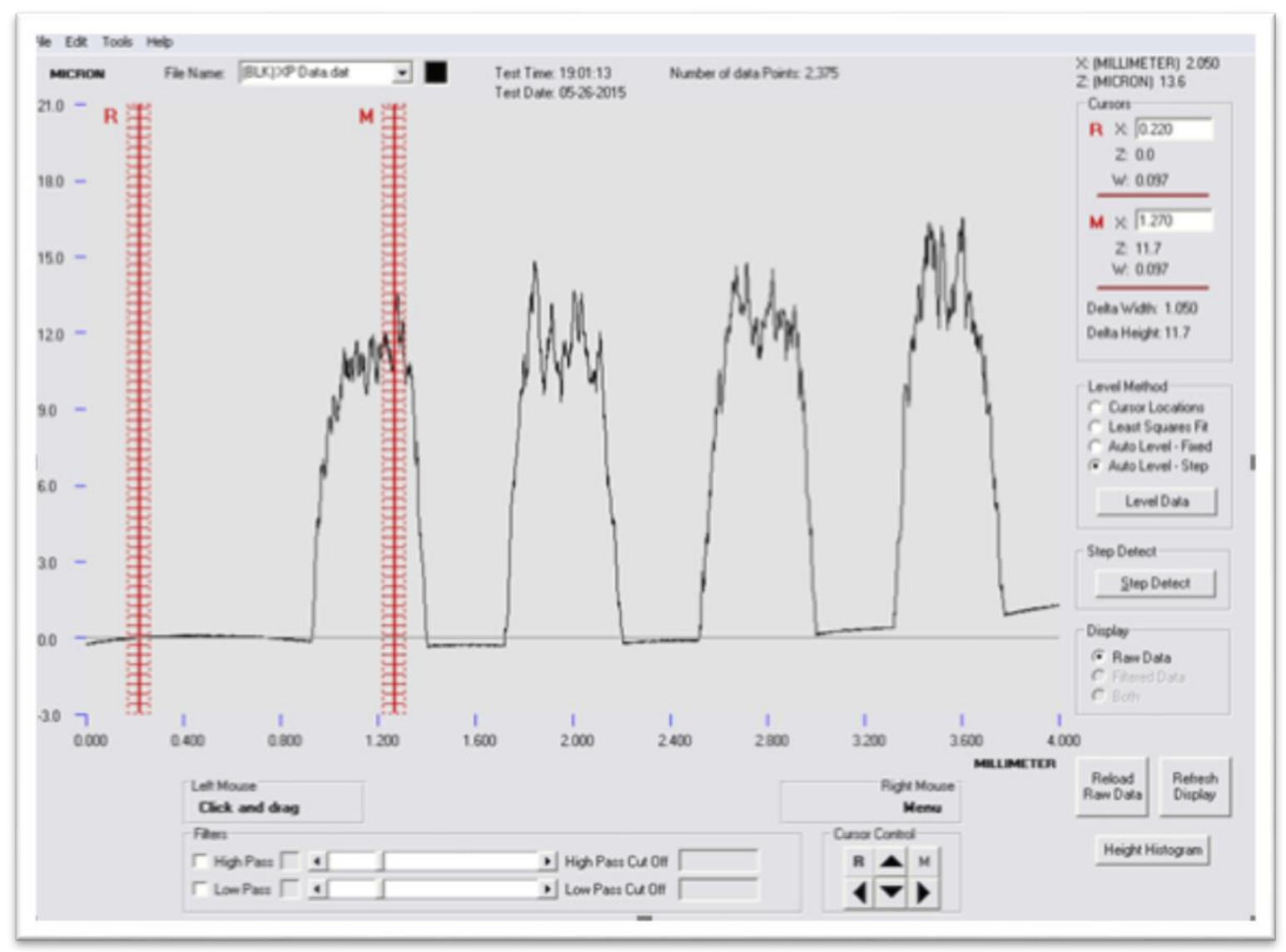

Figure 3.24 Profilometer Data of Printed Trace Sample

\subsection{Microfabrication Process}

The first step in the microfabrication process was to develop a mask of the design you want. The mask, seen below in Figure 3.25, was designed using AutoCAD and printed by a company called CAD Art Services, seen below in Figure 3.26. 

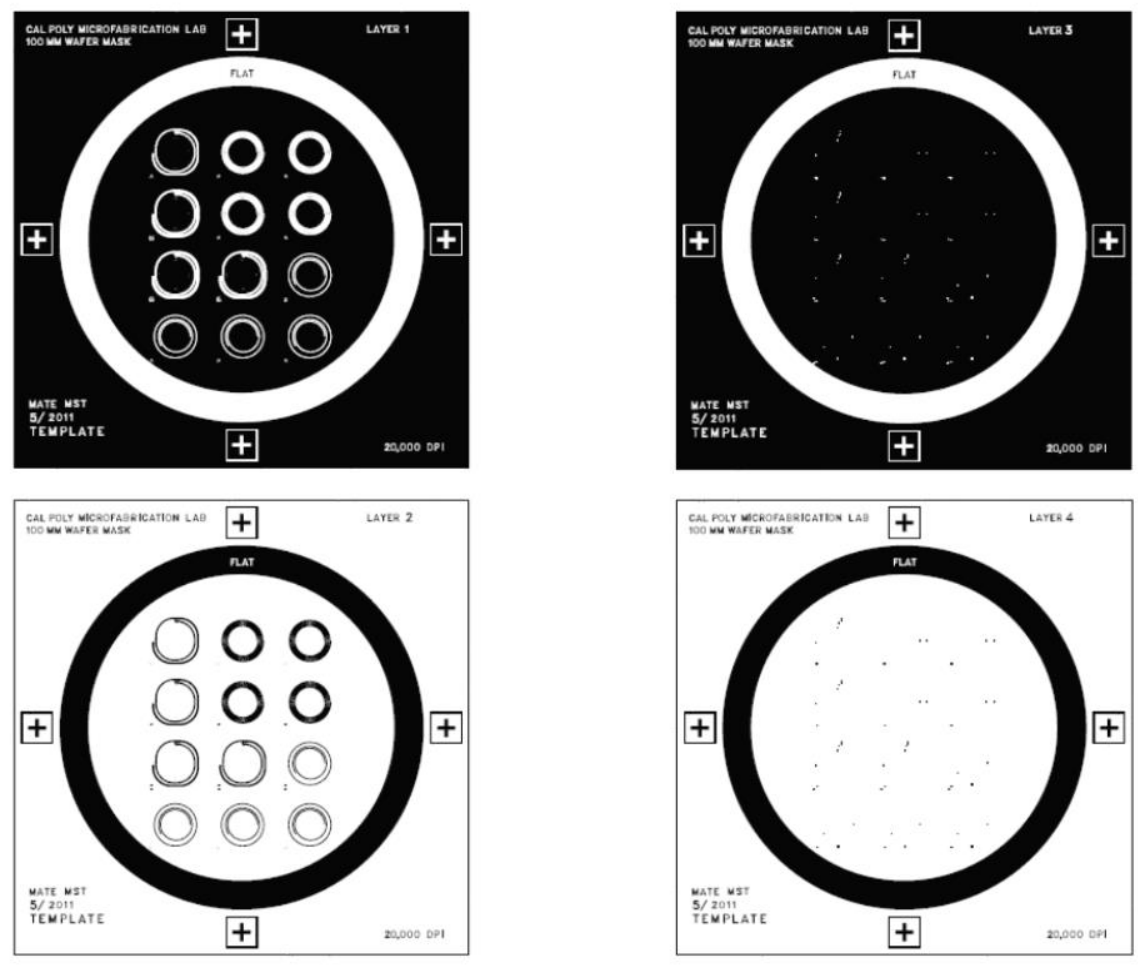

Figure 3.25 AutoCAD Design of Antennas

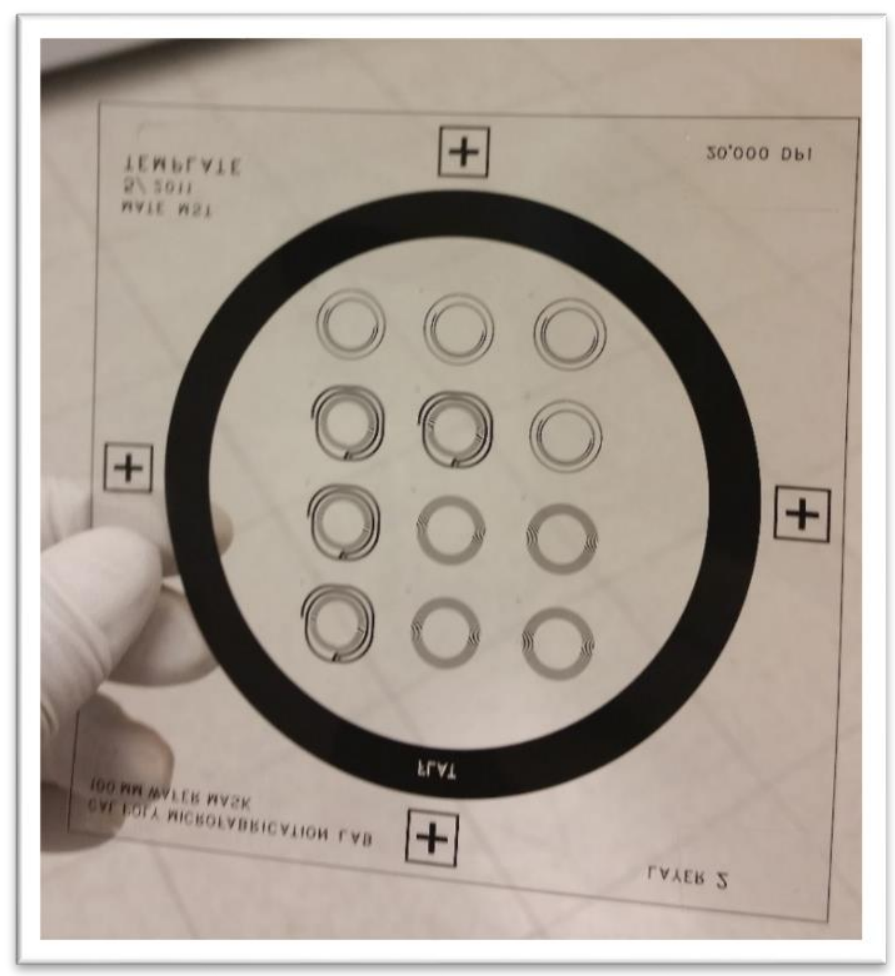

Figure 3.26 Printed Mask of Design 
The mask needed to be shaped and attached to a square piece of glass. The resulting completed mask can be seen in Figure 3.27.

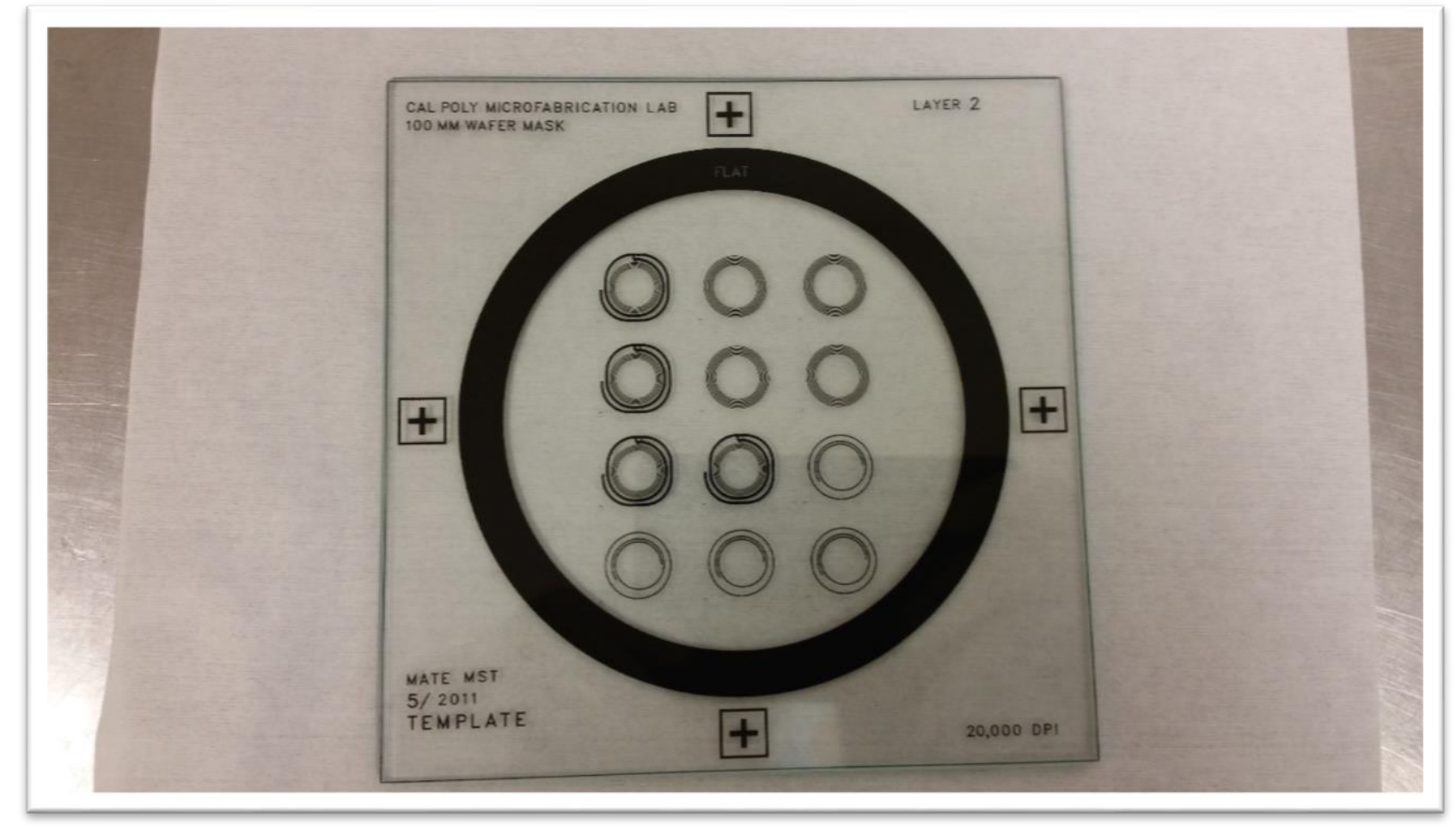

Figure 3.27 Completed Mask for Photolithography

The initial step is to select wafers to be processed which was an n-type, $100 \mathrm{~mm}$, $<100>$ plane wafer. 


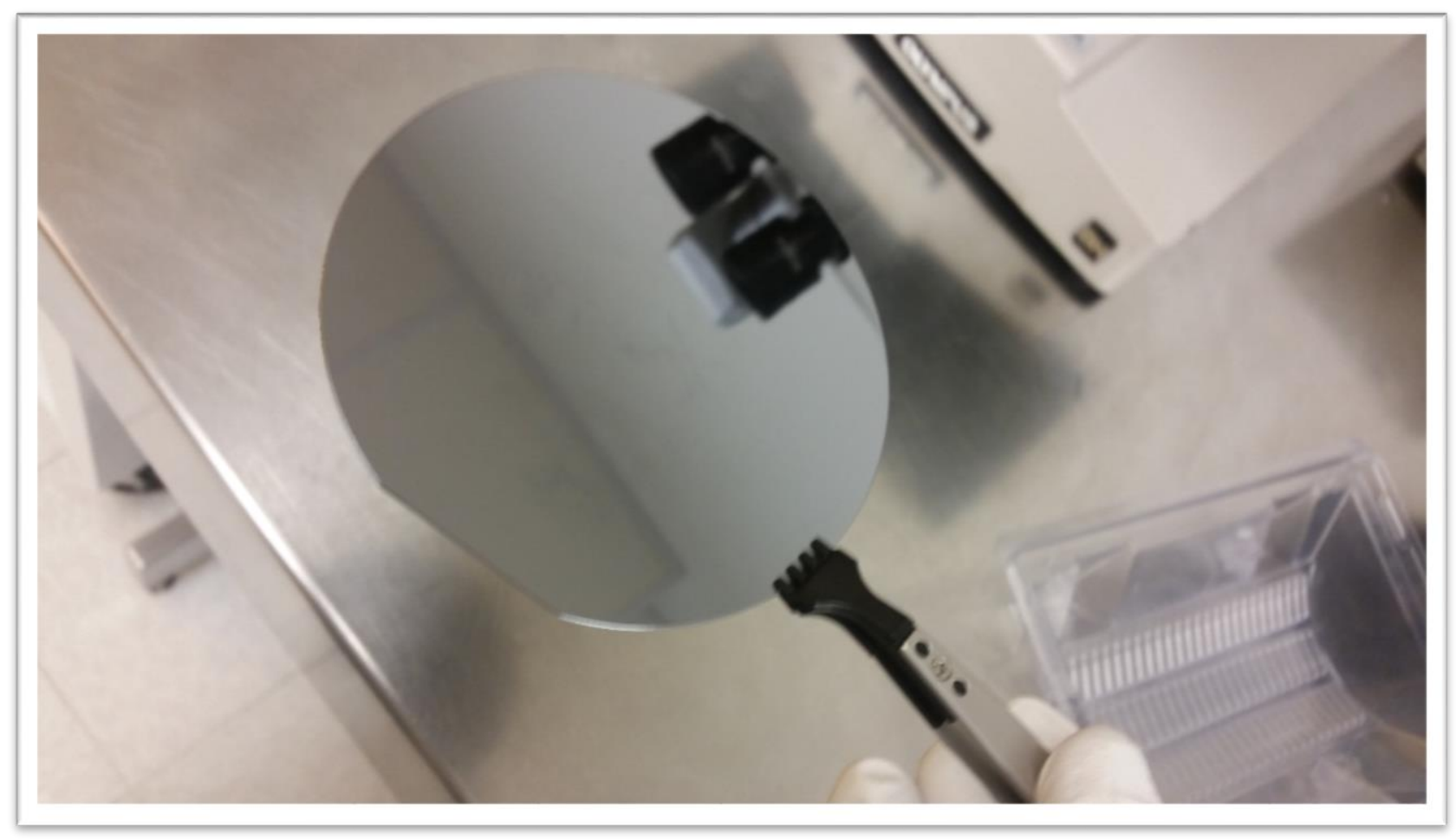

Figure 3.28 N-Type Wafer

The wafers are placed in a Teflon boat and submerged in piranha (a solution of sulfuric acid and hydrogen peroxide) at a temperature of 70 degrees Celsius for 10 minutes. This removed all the surface organics that could potentially cause contamination in the process. After 10 minutes in an acid bath the wafers were submerged in deionized (DI) water and placed in a buffer oxide etchant (BOE) bath for 30 seconds which consisted of hydrofluoric acid (HF), ammonium fluoride $\left(\mathrm{NH}_{4} \mathrm{~F}\right)$, and water $\left(\mathrm{H}_{2} \mathrm{O}\right)$. This step removed the oxide layer created naturally from the wafer reacting with the gases in the environment. After submerging the wafers in BOE the wafers were submerged in DI water again and then inserted in the rinse dryer machine. 
The total quantity made for this process was a $33 \mathrm{~mL}$ batch of PDMS mixture consisting of a base and curing agent that was had a 10:1 ratio. For a $33 \mathrm{~mL}$ the mixture consisted of $30 \mathrm{~mL}$ of base solution and $3 \mathrm{~mL}$ of curing agent.

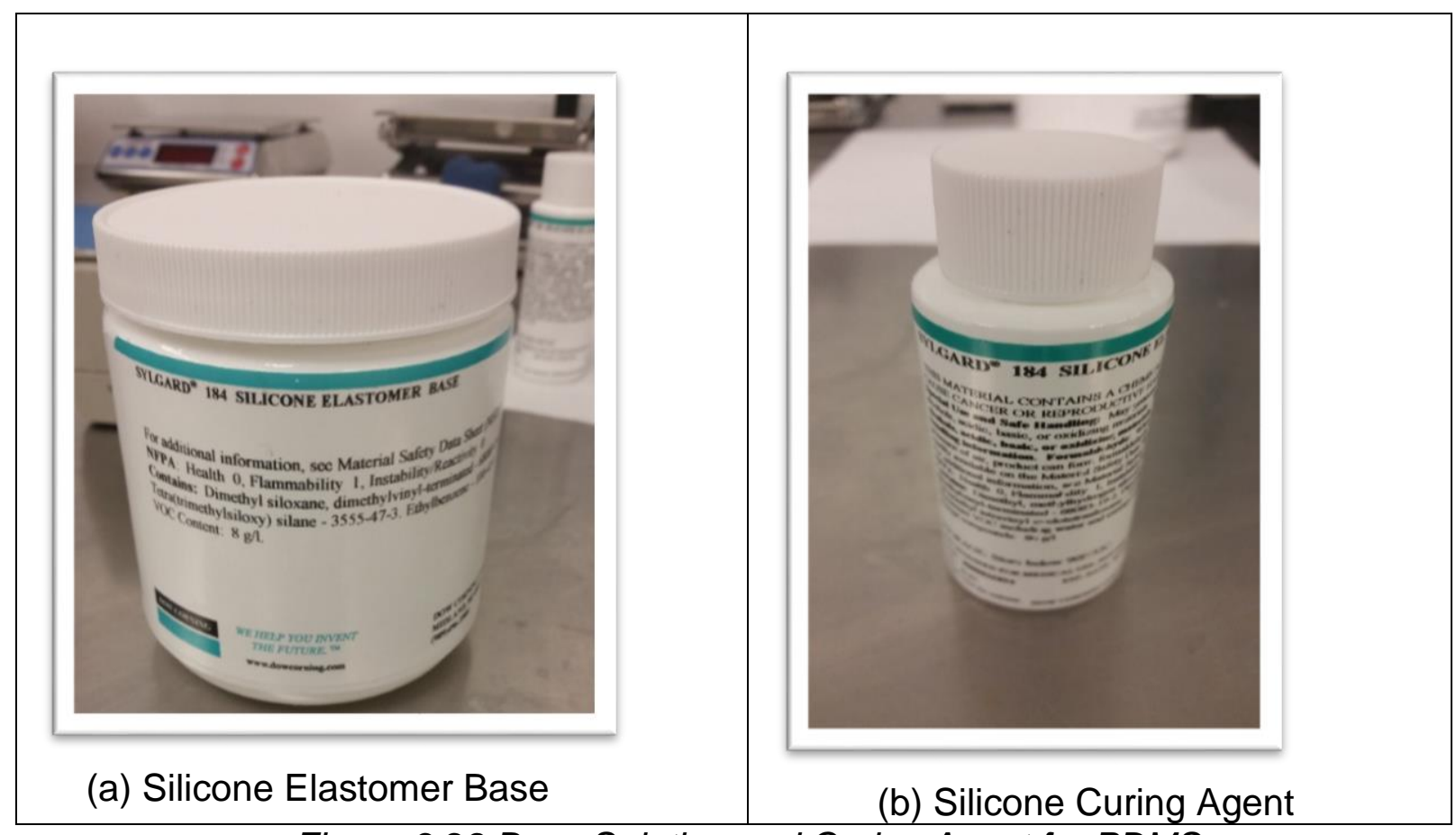

Figure 3.29 Base Solution and Curing Agent for PDMS

Using available syringes, the PDMS components were dispensed into a plastic mixing/measuring cup. The components were then stirred thoroughly and slowly for several minutes to avoid introducing excess oxygen into the mixture. 


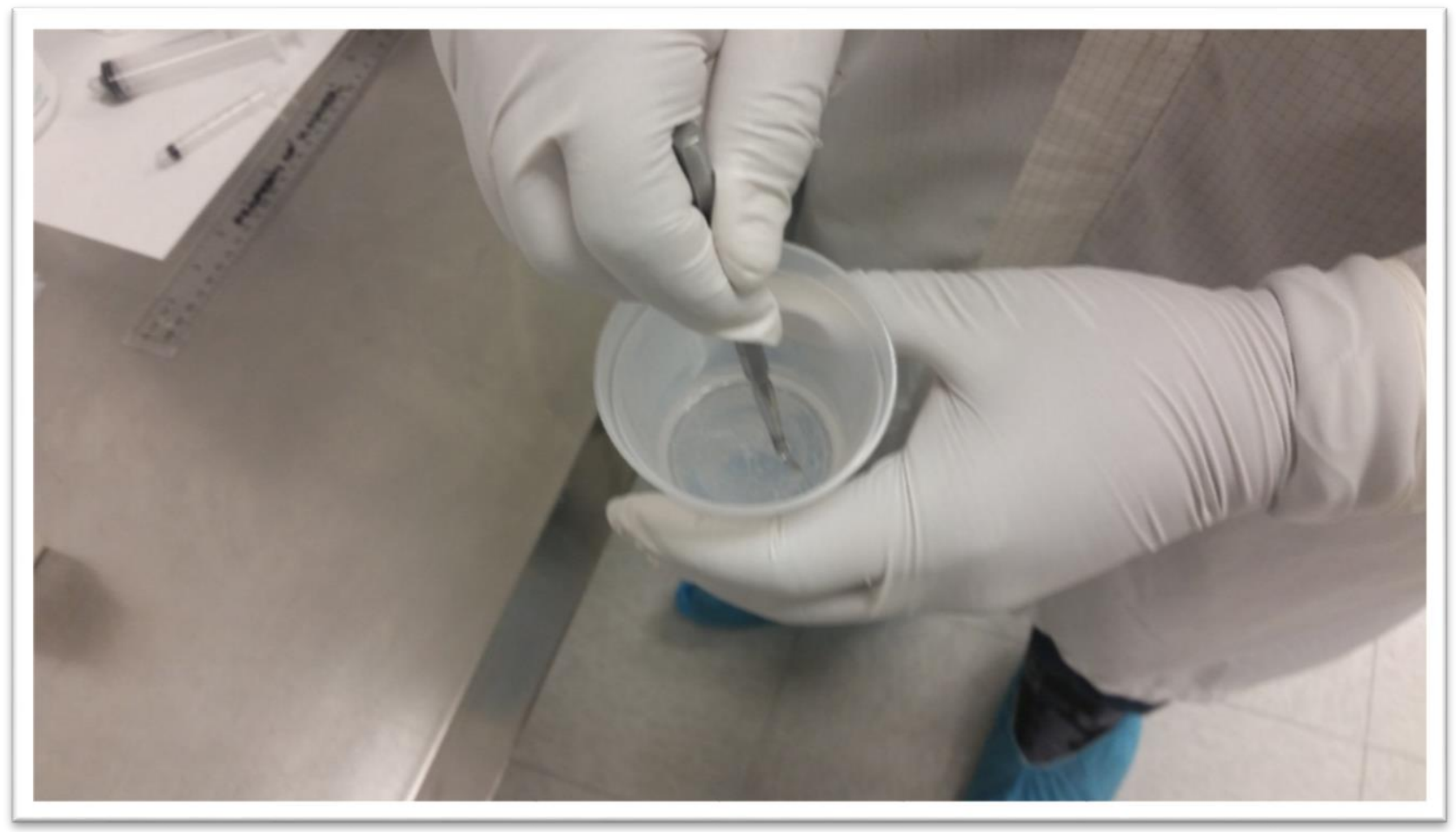

Figure 3.30 Mixture of Base and Curing Agent Solution in Cup The next step in the process was to place the PDMS in a vacuum chamber for degassing. With the vent valve and vacuum line valve closed, the vacuum pump was turned on from the utility chase. 


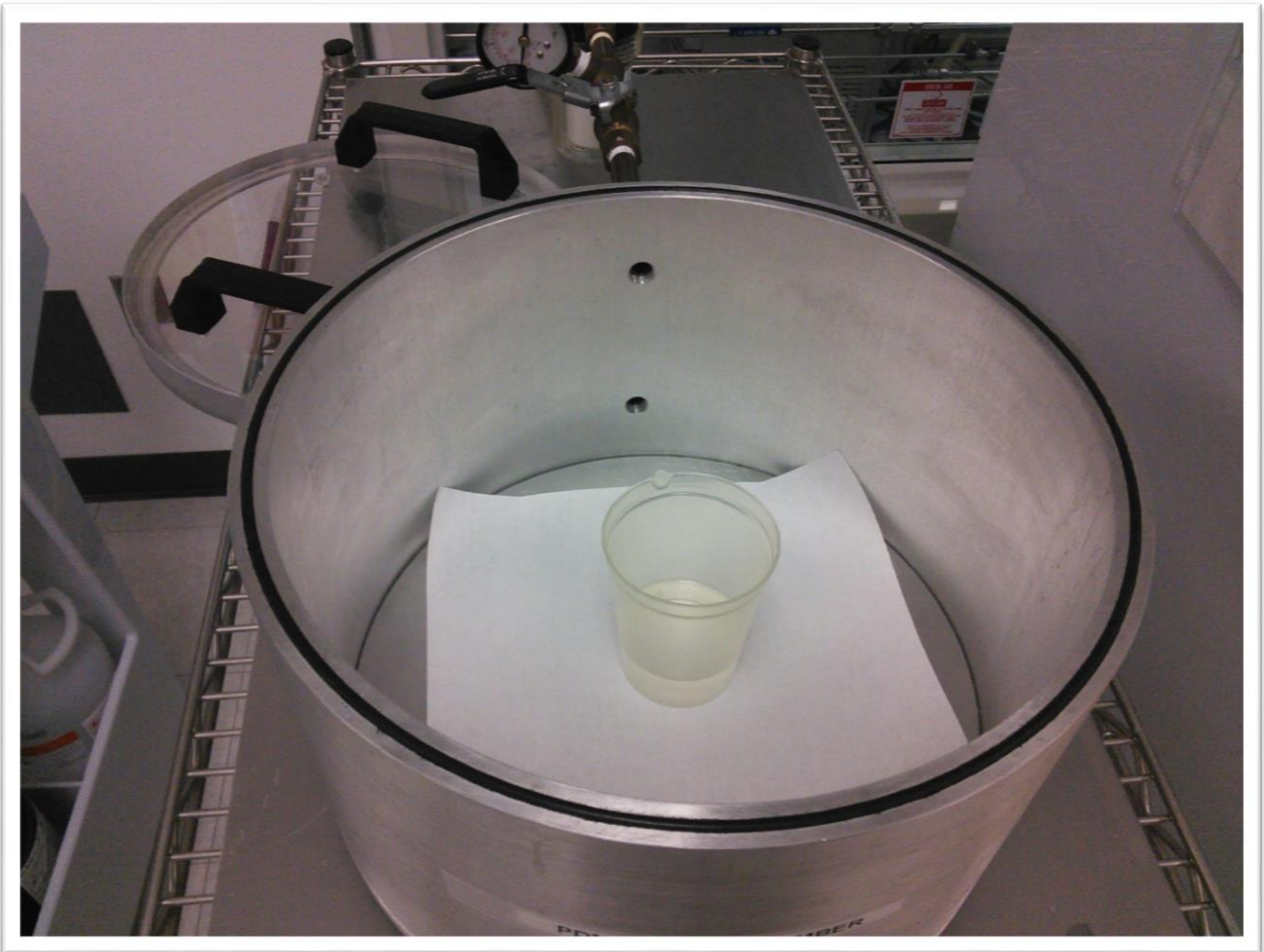

Figure 3.31 Solution in Degas Chamber

The vacuum line valve was then opened and chamber pumped down until large bubbles come out of PDMS. During the pump down step it is a good idea to throttle chamber pressure using the vent valve so that PDMS doesn't overflow the cup in the chamber. 


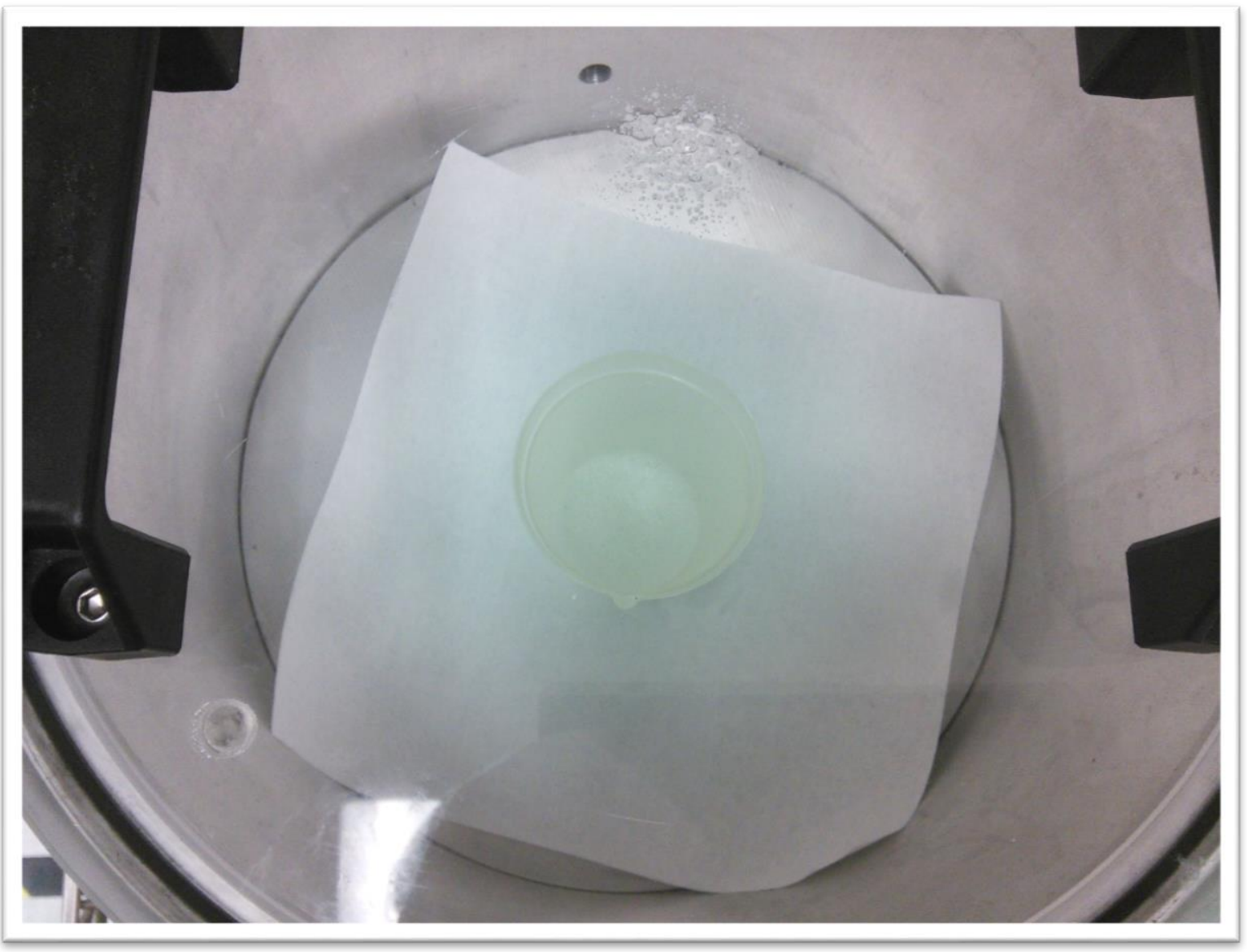

Figure 3.32 Being Degassed with rising bubbles

Overall degassing generally took anywhere from 10-20 minutes in order to ensure all the bubbles were removed from the mixture. Next the vacuum line valve was closed and the chamber was slowly vented back to atmospheric pressure. The degassed PDMS was removed from the chamber and ready for use.

The spin coater was lined with aluminum as shown in Figure 3.33. 


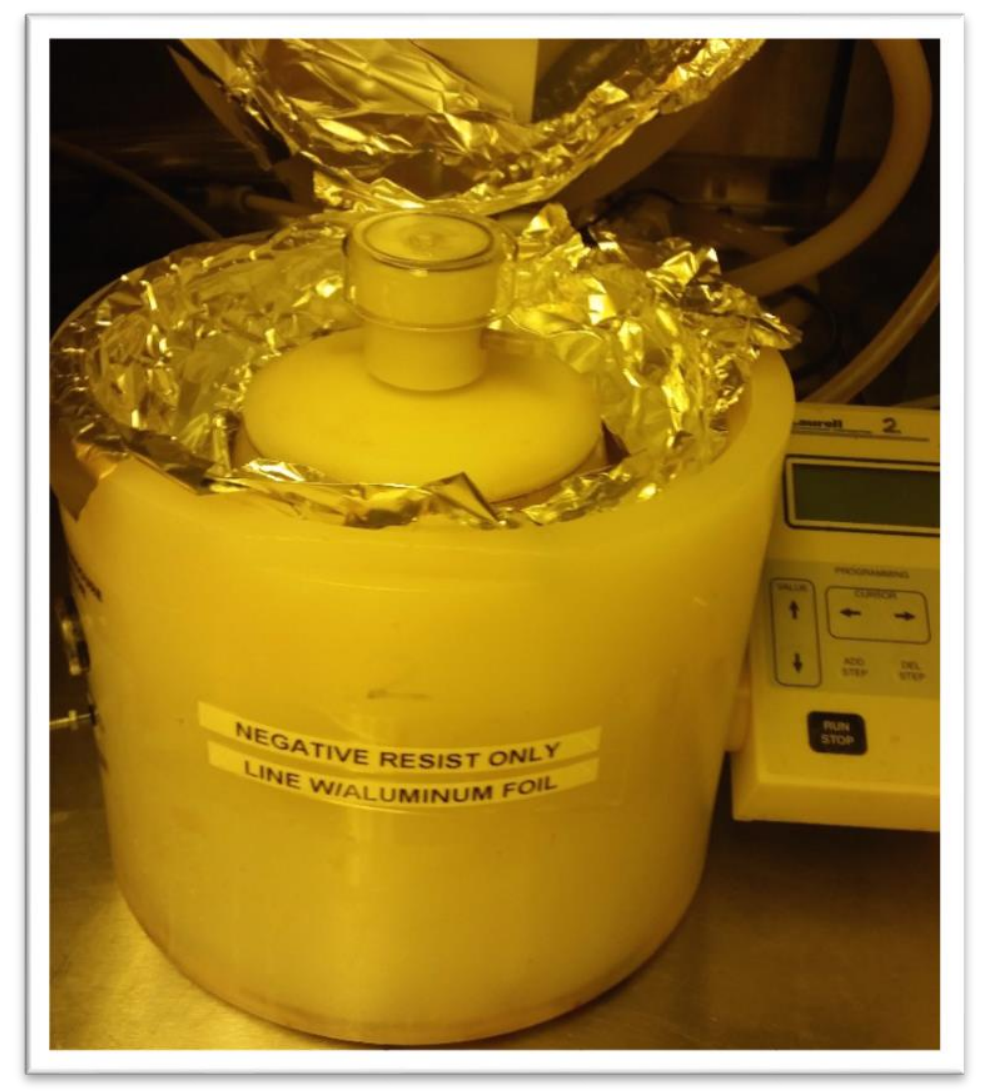

Figure 3.33 Aluminum Lined Spin Coater

The program for time of spin and RPMs were determined to result a 25um thick layer film. The data located in Appendix A from prior material engineering (MATE) students was referenced to determine the correct time and speed needed to achieve desired thickness. According to the data a $25 \mu \mathrm{m}$ film of PDMS can be achieved if it was spun for 60 seconds and a speed of 2500RPM. After spin coating the PDMS was partially cured at room temperature for 12 hours in a sealed container. The PDMS should have a gel like state when partially cured. An $85 \mathrm{~mm}$ diameter circular disk of $25 \mathrm{um}$ thick PET was centered on the thin film of PDMS. It was found through previous experiments of attempting to bind PET with PDMS that they will not bond together even with plasma bonding the two materials. With the PDMS in a gel-like state the PET 
will temporarily bind where another layer of PDMS covered the top of the wafer and the PDMS edges. The wafer was placed in an oven at 75 degrees Celsius for 2 hours for complete thermo-curing. Figure 3.34 below shows the resulting cross-sectional structure after thermos-curing.

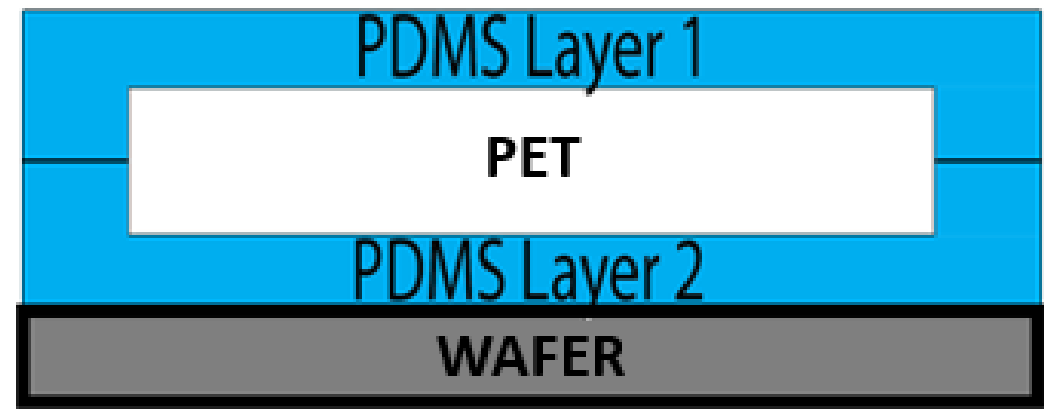

Figure 3.34 PDMS-PET Start Substrate for Processing the Contact Lens The next step was placing metal onto the substrate by means of physical vapor deposition (also known as sputtering). The following procedure was used for sputtering:

1) The following gas tanks were turned on: High Pressure Argon, High Pressure Nitrogen, and Low Pressure Nitrogen gas. 


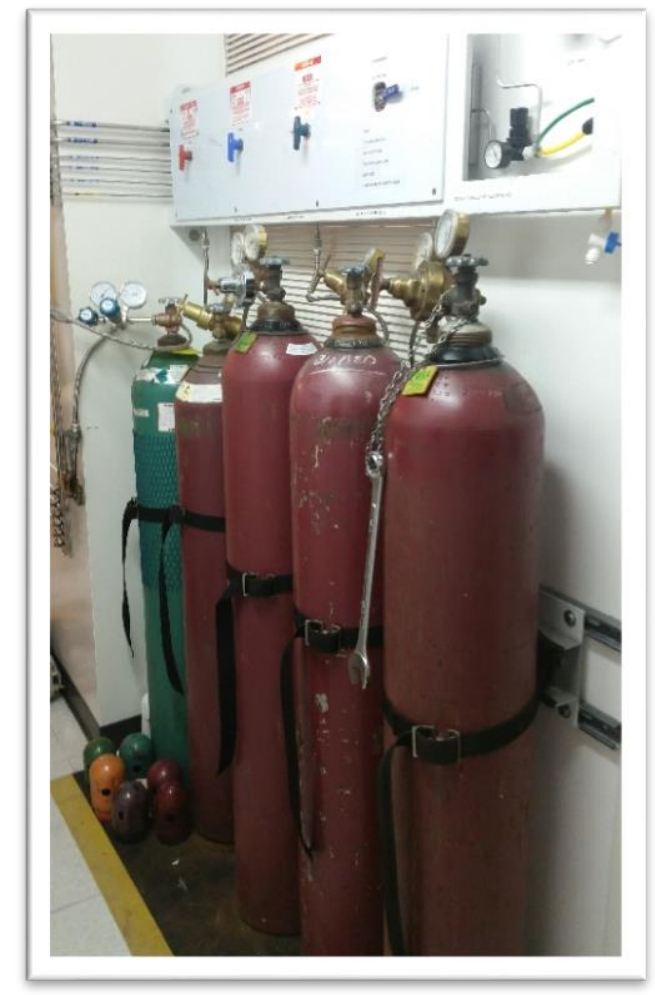

Figure 3.35 Gas Tanks Need for Sputtering Process

2) The molds were placed inside the sputtering machine, the Denton Vacuum, as seen in Figure 3.36.

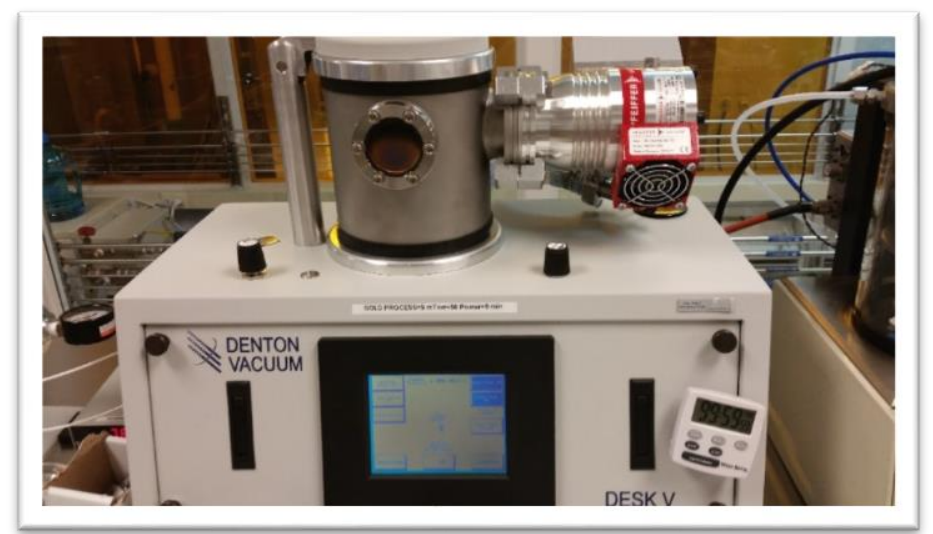

Figure 3.36 Denton Vacuum PVD Machine

3) The gold target was installed in the machine as seen in Figure 3.37. 


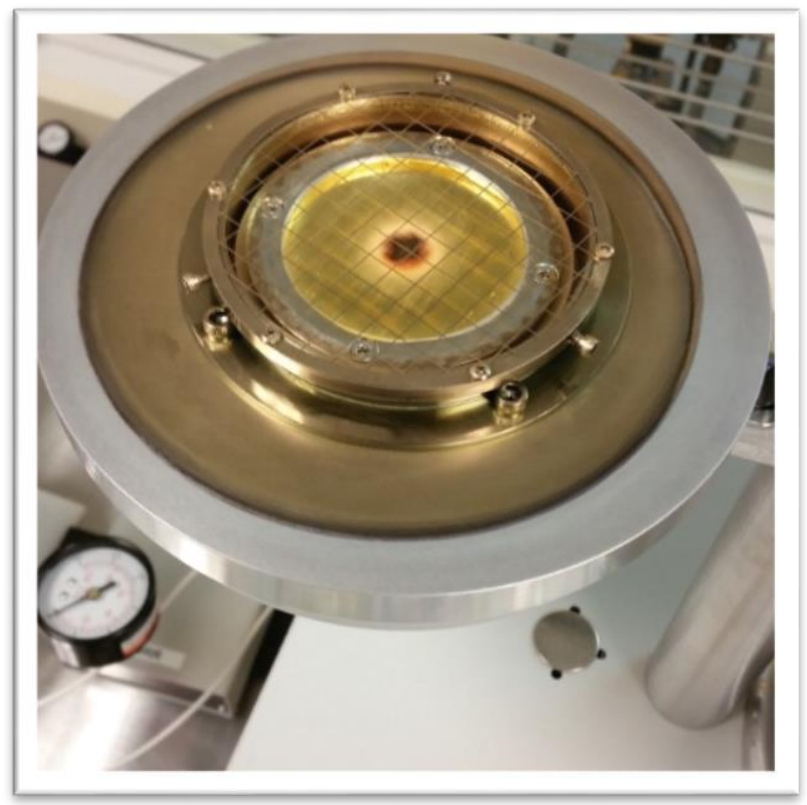

Figure 3.37 Gold Target for Sputtering

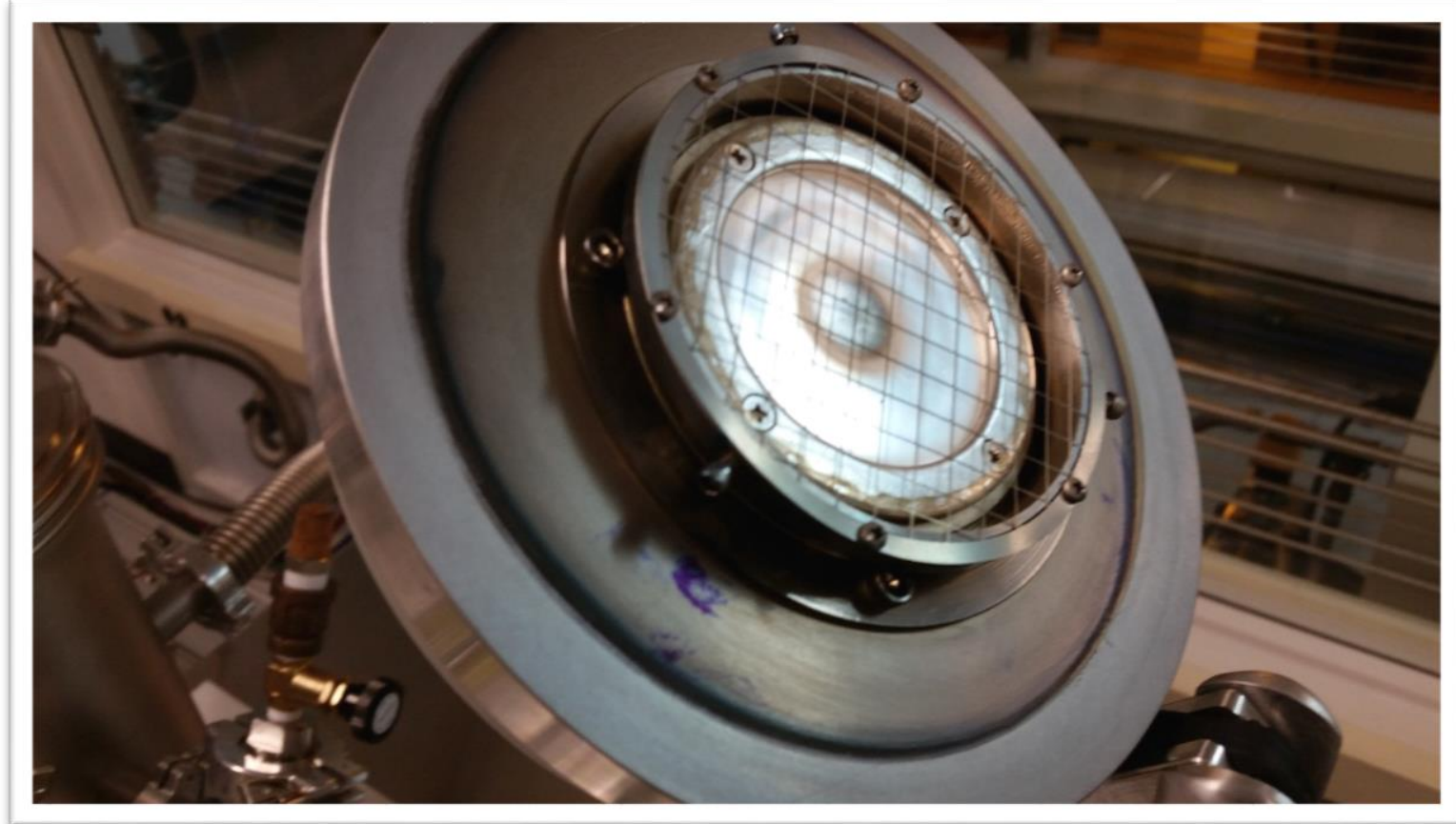

Figure 3.38 Silver Target for Sputtering

4) After installation the machine was turned on. The cured models were sprayed with nitrogen and placed in the chamber with a glass slide and a piece of tape to attach to the bottom of the rotating target platform. 


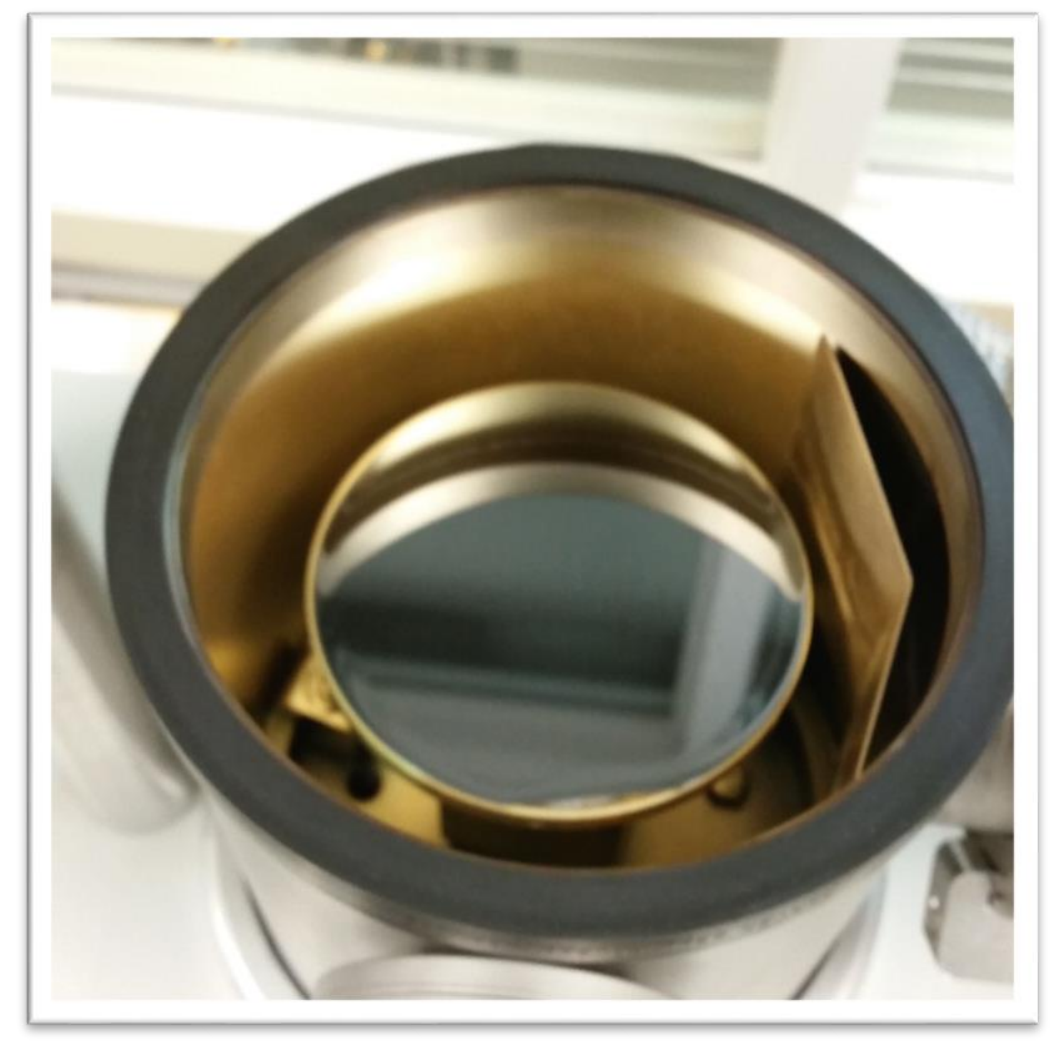

Figure 3.39 PDMS Wafer Placed in PVD Machine

5) Pressed the start menu on the touch screen and turned on the mechanical pump.

6) Waited until the pressure was at 12 torr to activate the turbo pump.

7) Waited until base pressure reached $1.90 \times 10^{-5}$ torr which took about one hour and 45 minutes.

8) Turned on the gas valve resulting in a pressure jump of 9 mtorr.

9) Waited 5 minutes and initiated rotation.

10) Turned on sputter power (on display, the DC Current reads $48 \mathrm{~mA}$ ).

11) Time of sputter was approximately 7 minutes.

12) Turned off sputtering, rotation, and auto vent to stabilize pressure to atmospheric pressure. This step has a 600 second count down and an additional 5 minute wait time for venting. Figure 3.40 shows the result of sputtering. 


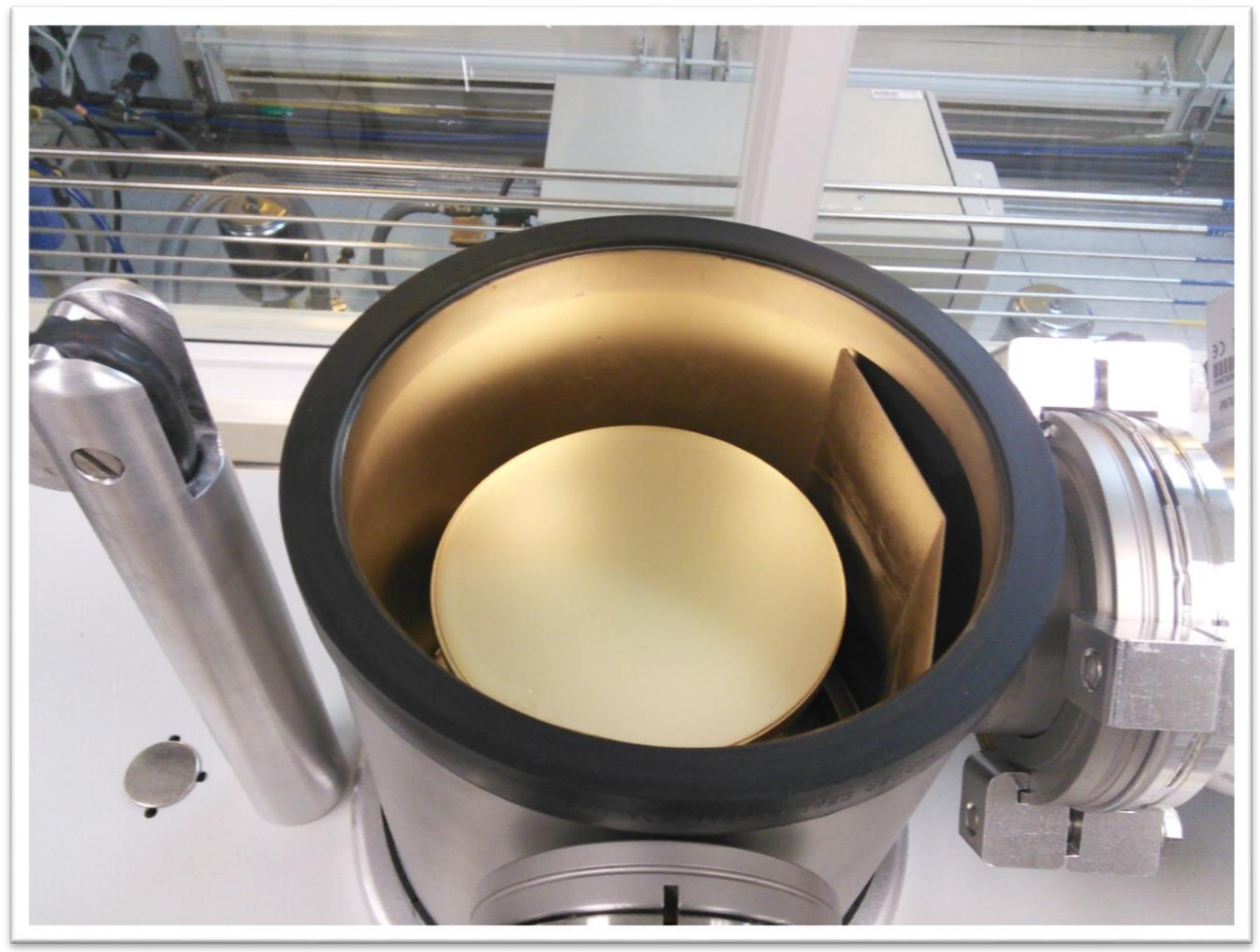

Figure 3.40 Wafer with Sputter Gold

Next the positive photo resist was spin coated onto the wafer to initiate the patterning process. A different spin coater was used with program setting MST_MATE_LOGO. The spin speed and times used are the same used for making PMOS transistors in the microfabrication process traveler [30]. 


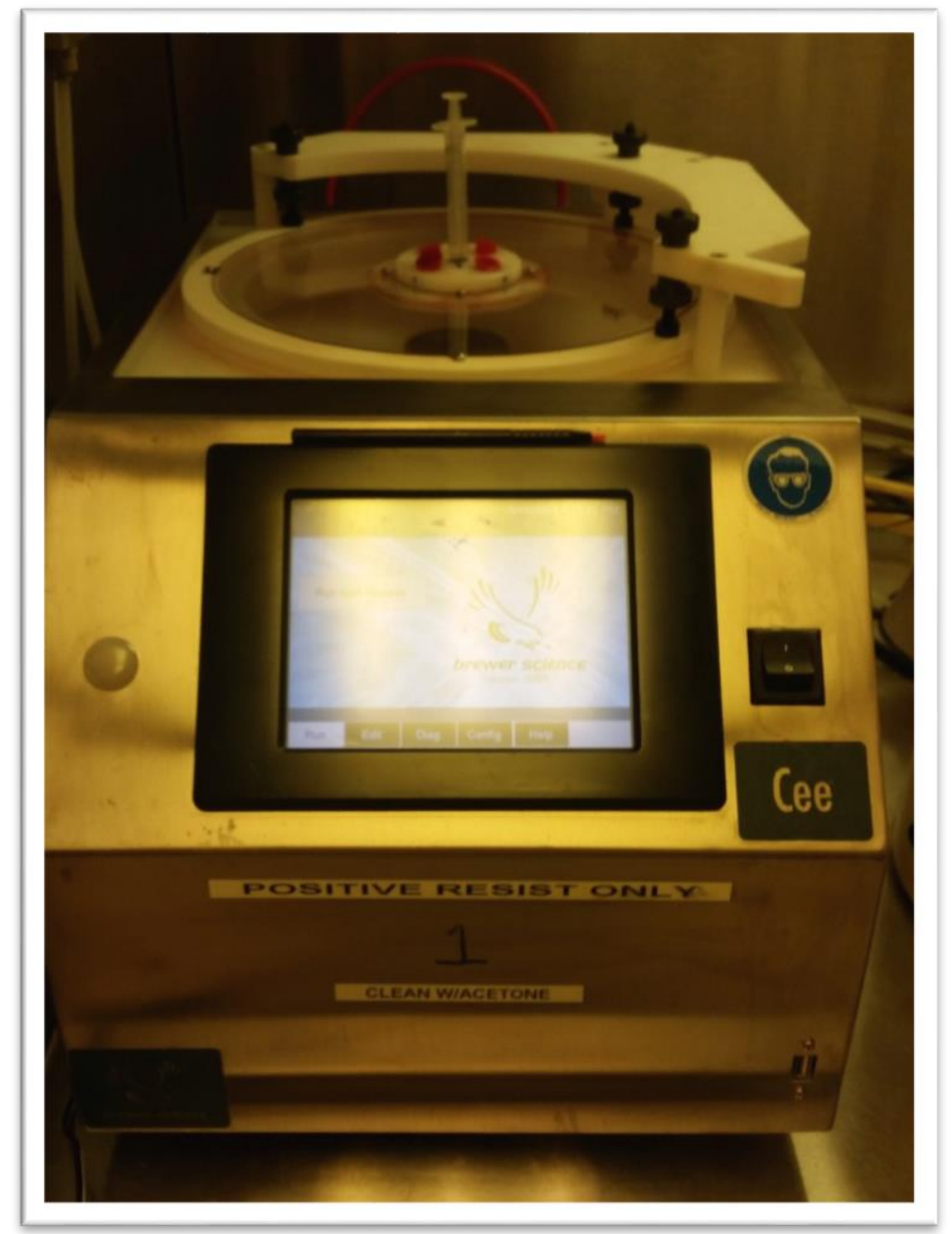

Figure 3.41 Positive Resist Spin Coater Machine

The wafer is then soft baked at 90 degrees Celsius for 60 seconds and cooled for 30 seconds. The photolithography machine was set-up as follow:

1) The low purity nitrogen gas tank and vacuum were turned on.

2) The wafer was sandwiched between a square shape glass and mask. 


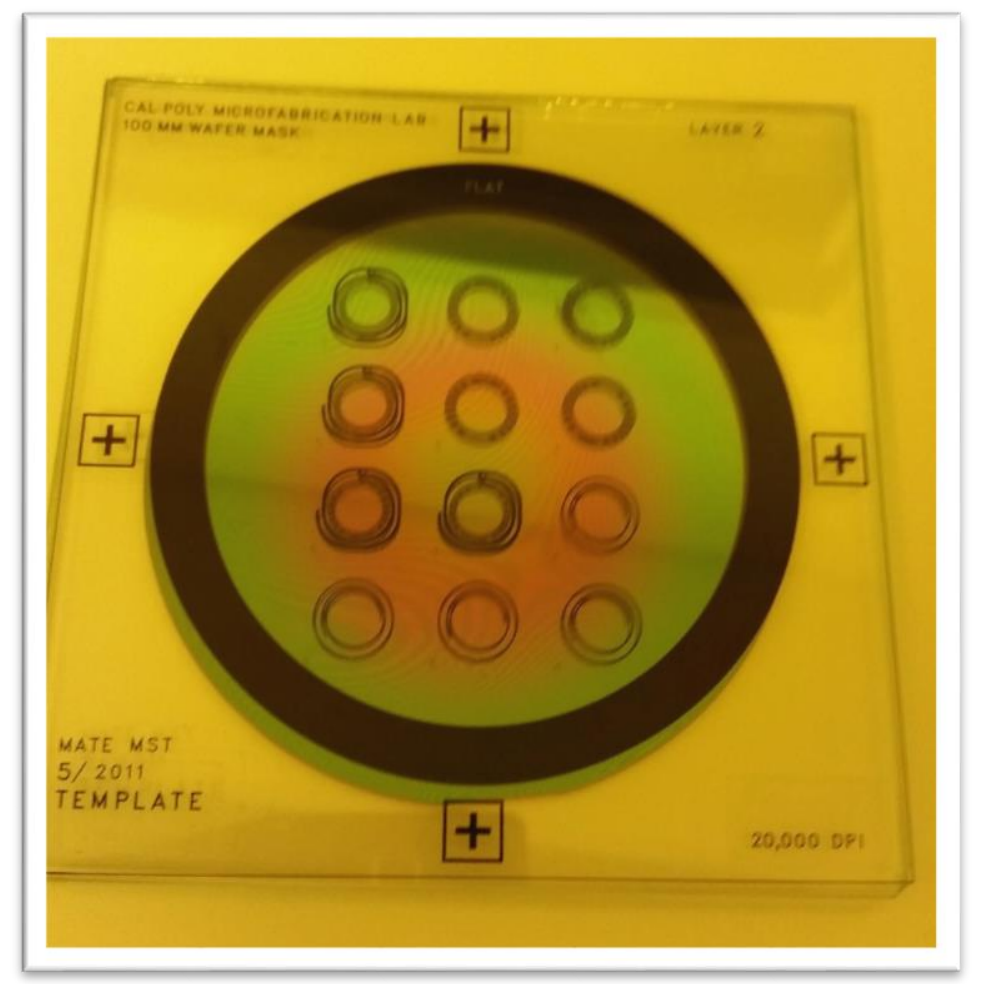

Figure 3.42 Wafer Sandwiched between Mask and Glass Slide Backing

3) The patterned mask with wafer was placed inside the machine, the cannon aligner as seen in Figure 3.43.

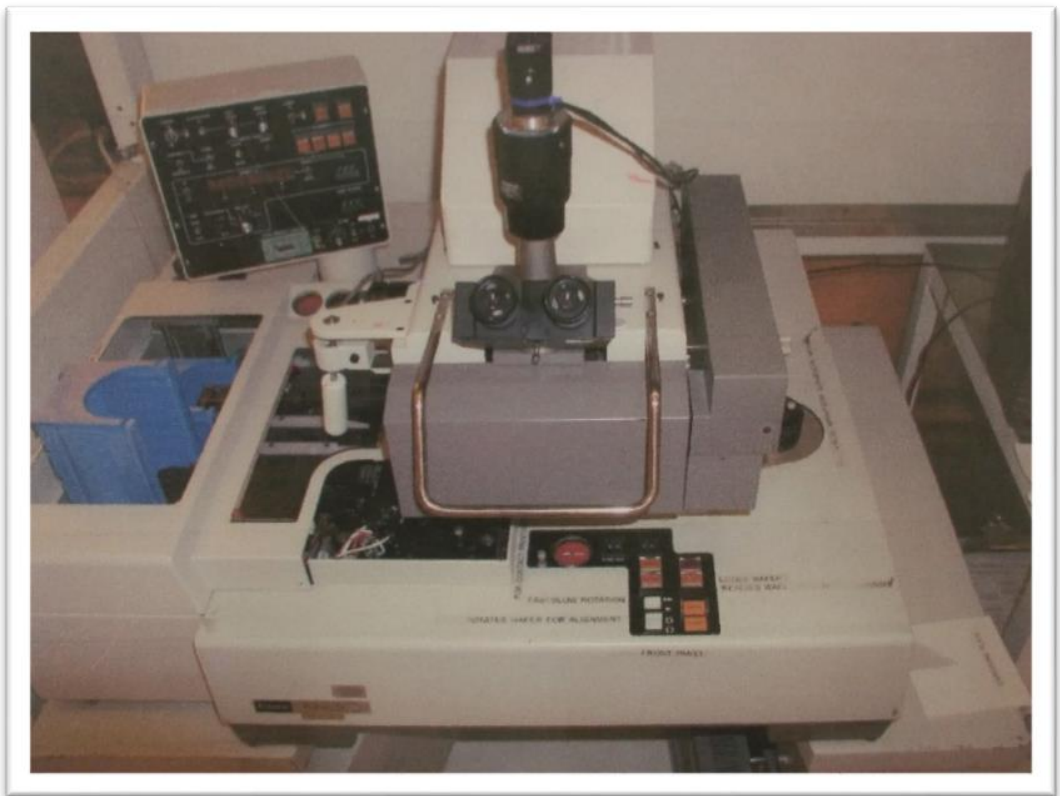

Figure 3.43 Canon Aligner for Photolithography 
4) The machine was then set to manual mode. This was because the wafer was too thick to fit in the mechanical loader and could possibly become stuck.

5) The light shutter was manually opened for 33-35 seconds. This time was determined by the Microchem datasheet where the total amount of energy to cause a reaction in the resist is $150 \mathrm{~mJ} / \mathrm{cm}^{2}$. The output power of the mercury bulb was $5 \mathrm{~mW} / \mathrm{cm}^{2}$. Resulting in the 30 seconds of time plus 10 percent.

After exposure, the wafer was developed for approximately 3 minutes in developer. The wafer was then hard baked for 60 seconds. This was changed to 2 minutes in the final version of the process to improve the percentage of antennas without facture defects. Gold etchant was used to remove the unwanted parts of the metal film. This took approximately 2 minutes. The wafer was then removed from the gold etchant and rinsed with DI water and dried with nitrogen. Figure 3.44 shows the patterned antennas protected by a layer of resist.

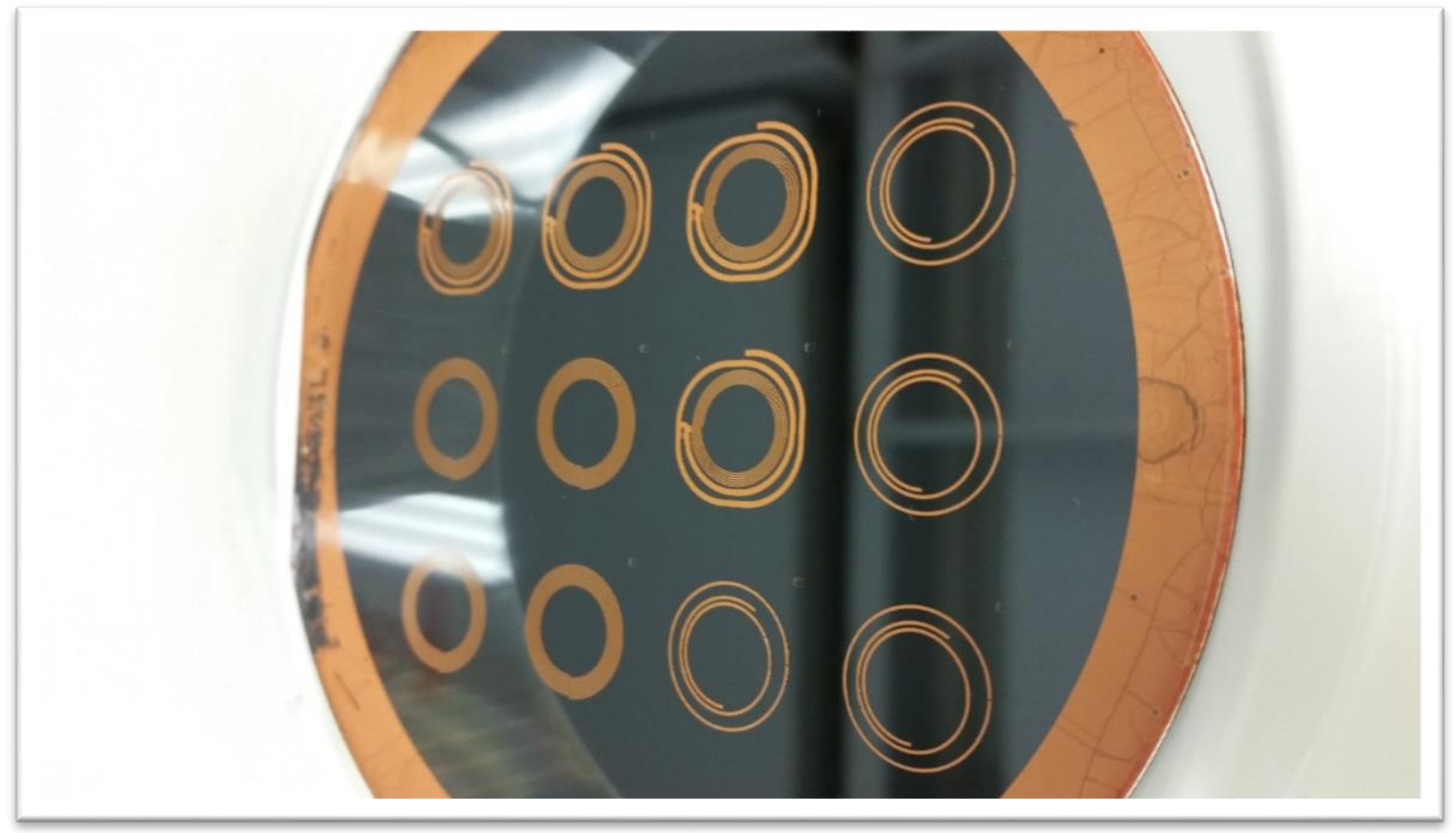

Figure 3.44 Pattern Antennas with Resist Layer 
Finally resist stripper was used to remove the remaining positive resist leaving the gold pattern antennas shown in figure 3.45.

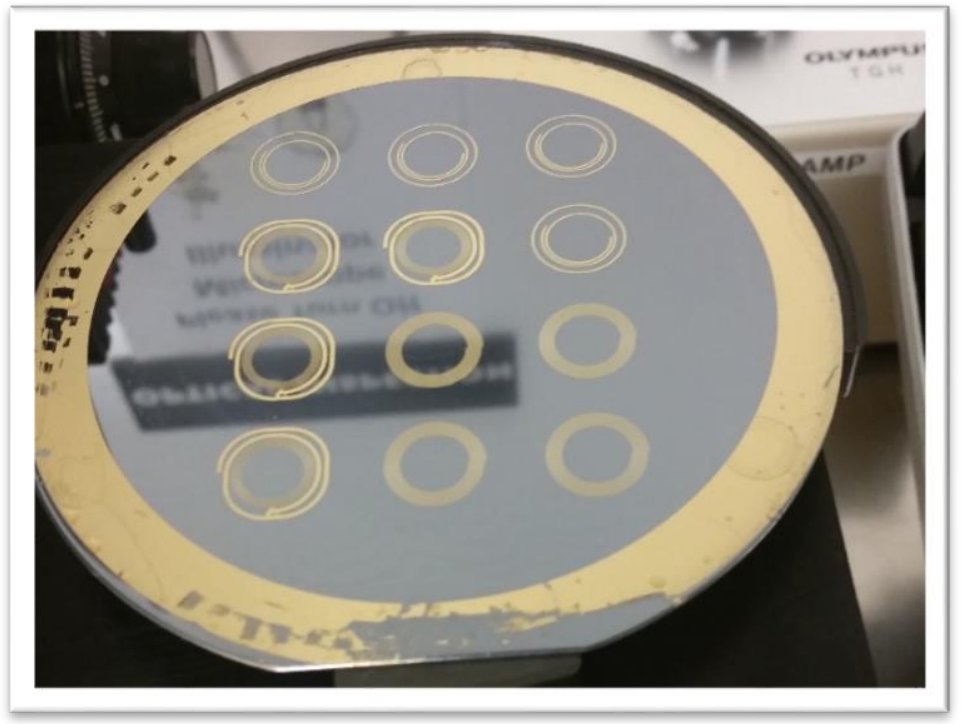

Figure 3.45 Patterned Gold 2.4 GHz/13.56MHz Antennas

The antennas were observed under an optical microscope to uncover defects.

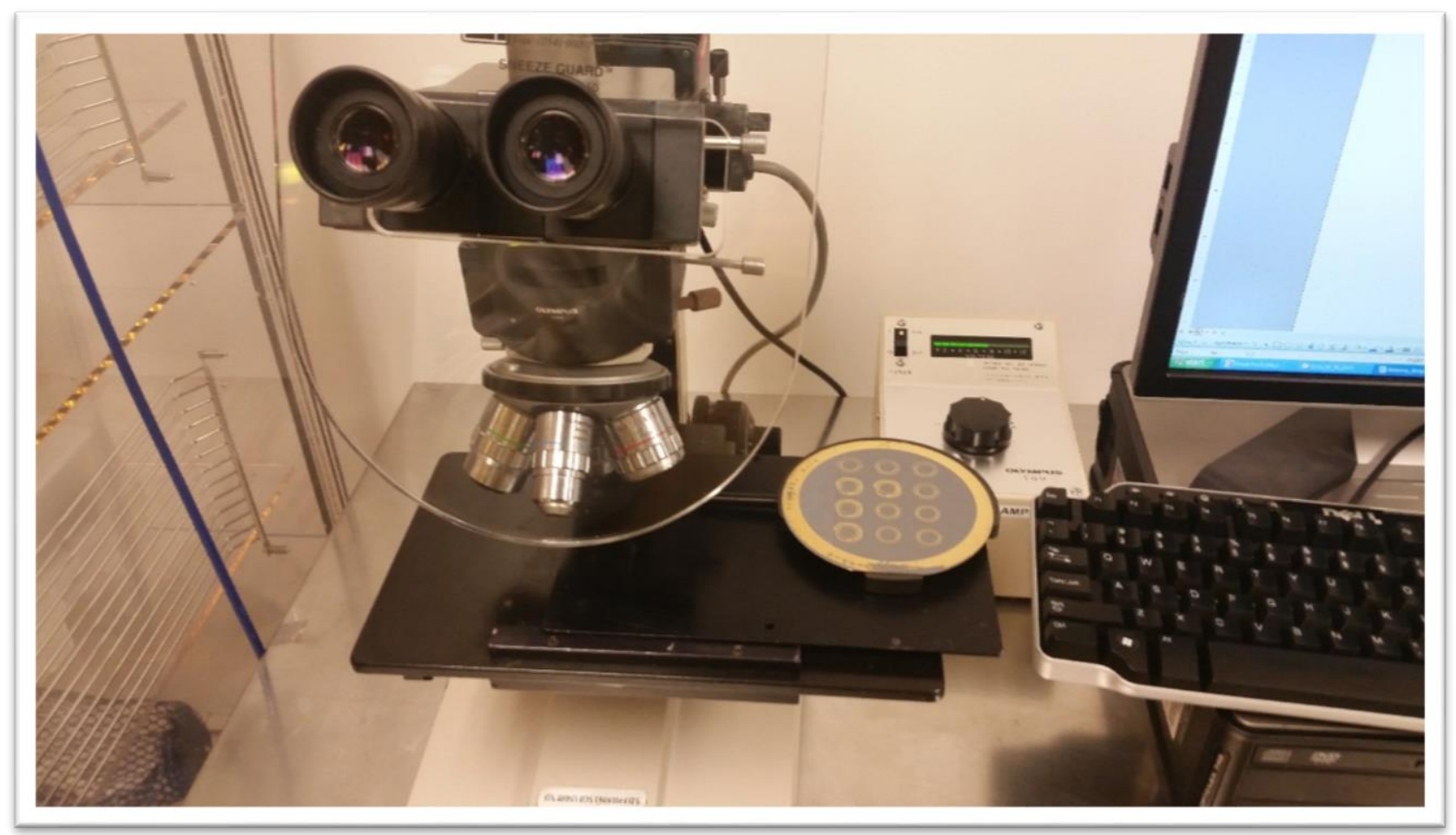

Figure 3.46 Optical Microscope for Analyzing Defects 
The following defects were found through observation and the cause determined for the particular defect.

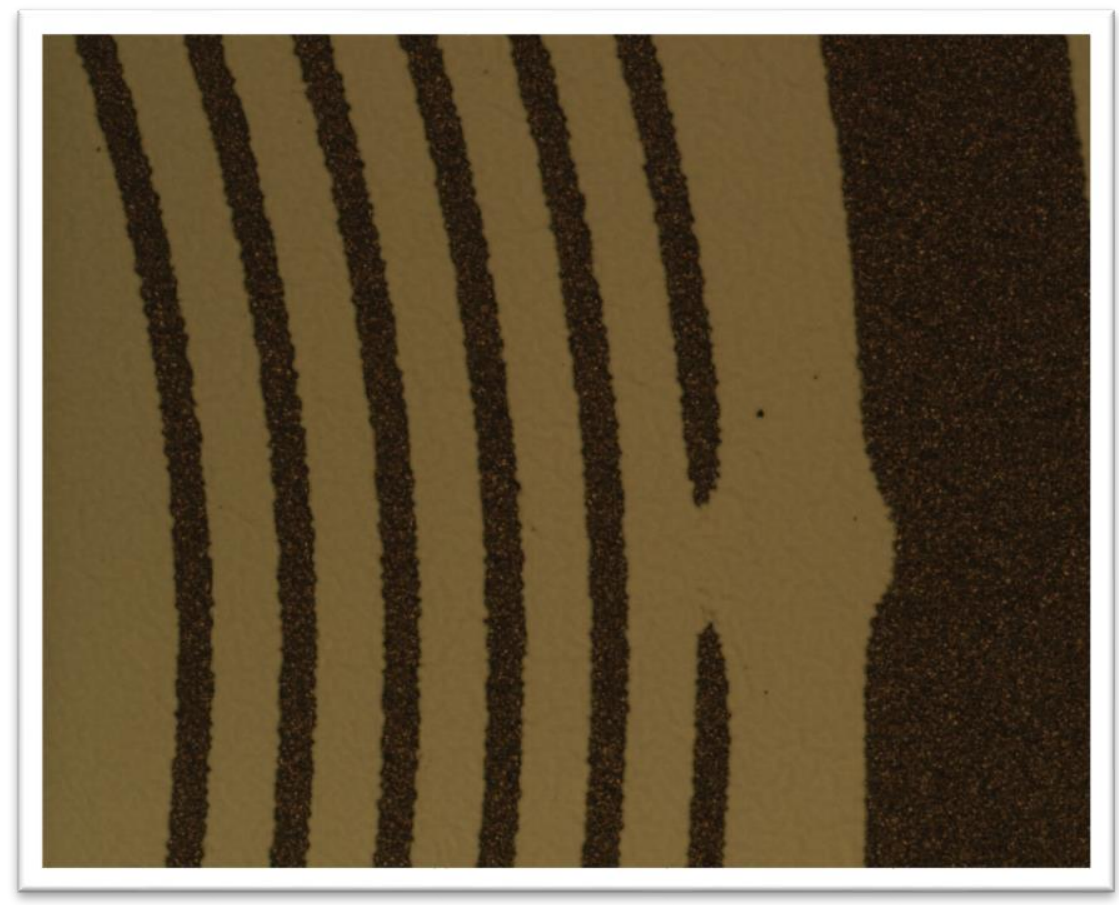

Figure 3.47 Trace Defect Due to Contamination

It was determined that organic particles played a role in this defect. This prevented the gold from attaching to the substrate in that location. The other defect identified was fracturing in the trace seen in Figure 3.48. These fractures were due to the swelling of the PDMS substrate during the developer and etchant process. One solution for minimizing factures was to increase the hard bake time of the photoresist. 


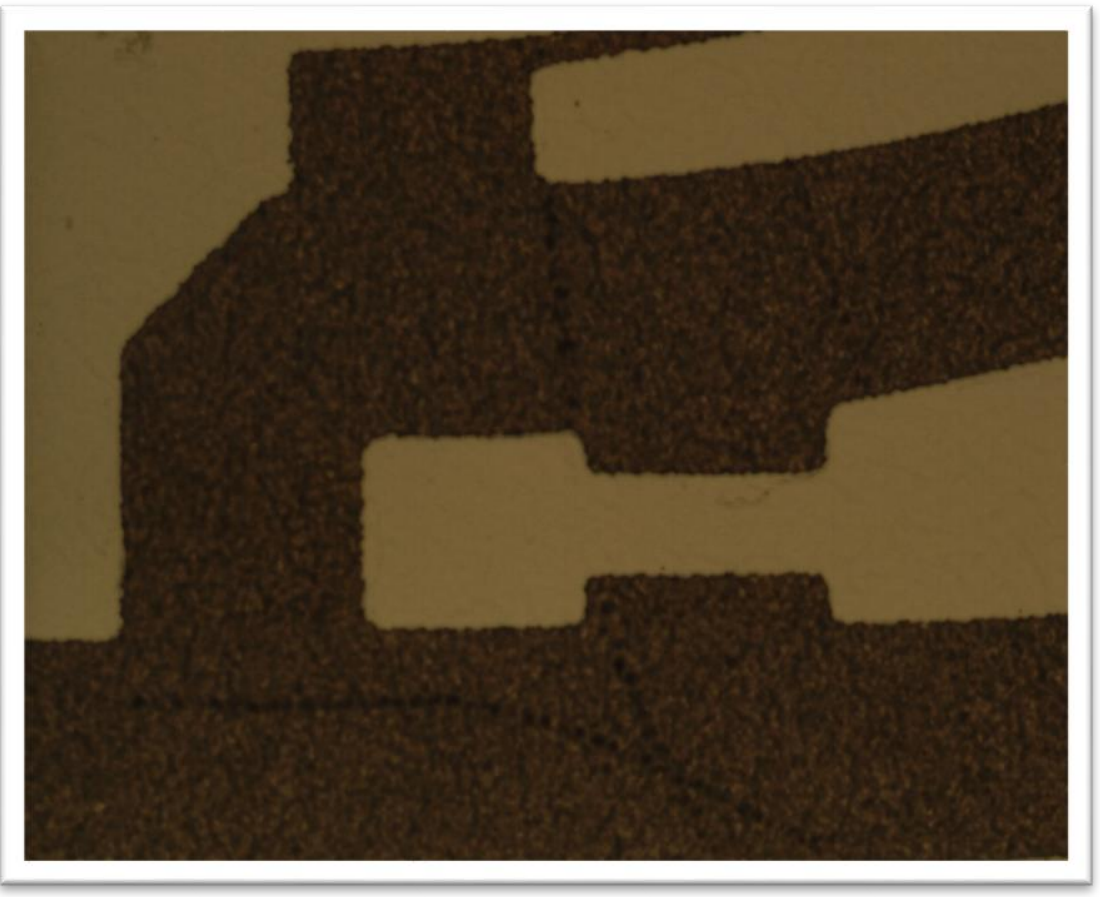

Figure 3.48 Trace Defect Fracturing

Antenna 1, 2, and 3 are shown in Figure 3.49 where the widths of the traces were measured. 


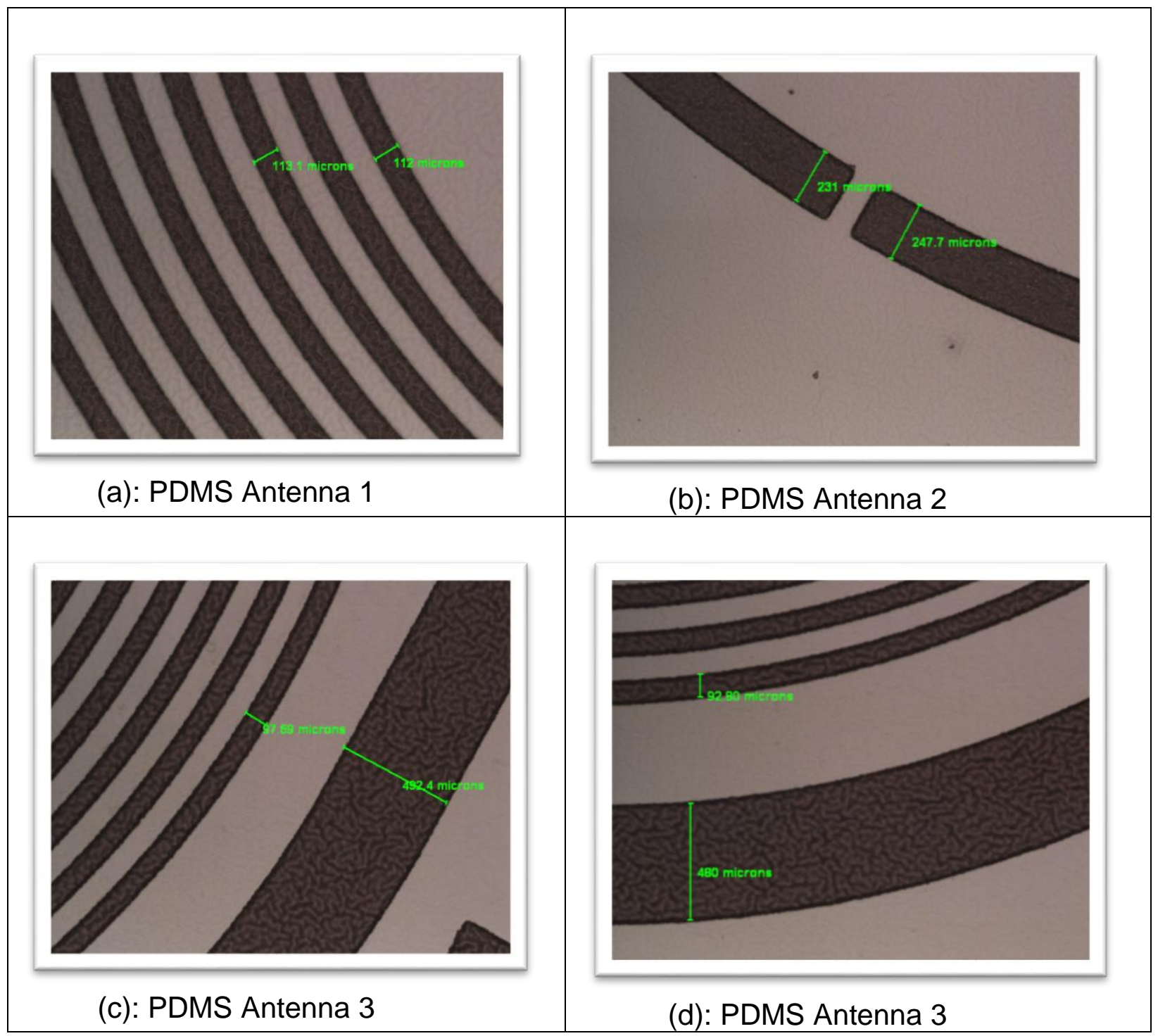

Figure 3.49 Width Measurements of Antenna 1, 2, and 3

The widths were within the desired range of feature sizes. Antenna 1 in Figure 3.49(a) was about 4-5um less than the designed width, antenna 2 in Figure 3.49(b) was 3-19um under the designed width, and Figure 3.49(c) and (d) are 8um less of the designed width for the inner coils and 20um smaller width on the outer trace.

The individual dies were separated from the wafer by using a diamond tip scribe, and a paper clip. Unfortunately, there were no die cutting saws small 
enough for the clearance of each die and could potentially become contaminated by oil leaking on to the wafer. Laser cutting was another option, however, the two lasers located on campus did not have sufficient power to administer a clean cut into the wafer. The result of the die is shown in Figure 3.50 .

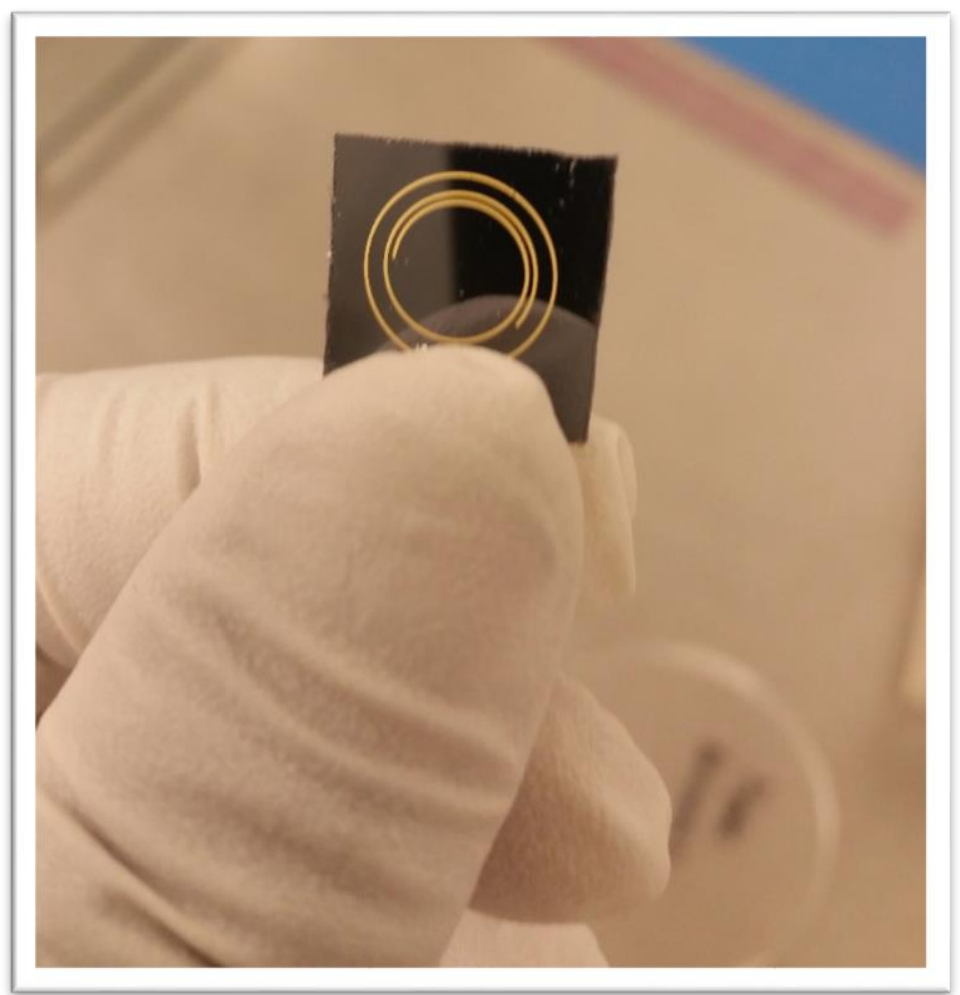

Figure 3.50 Cutout of Die

Next, the thickness of the traces in the z-direction were measured using a profilometer. 


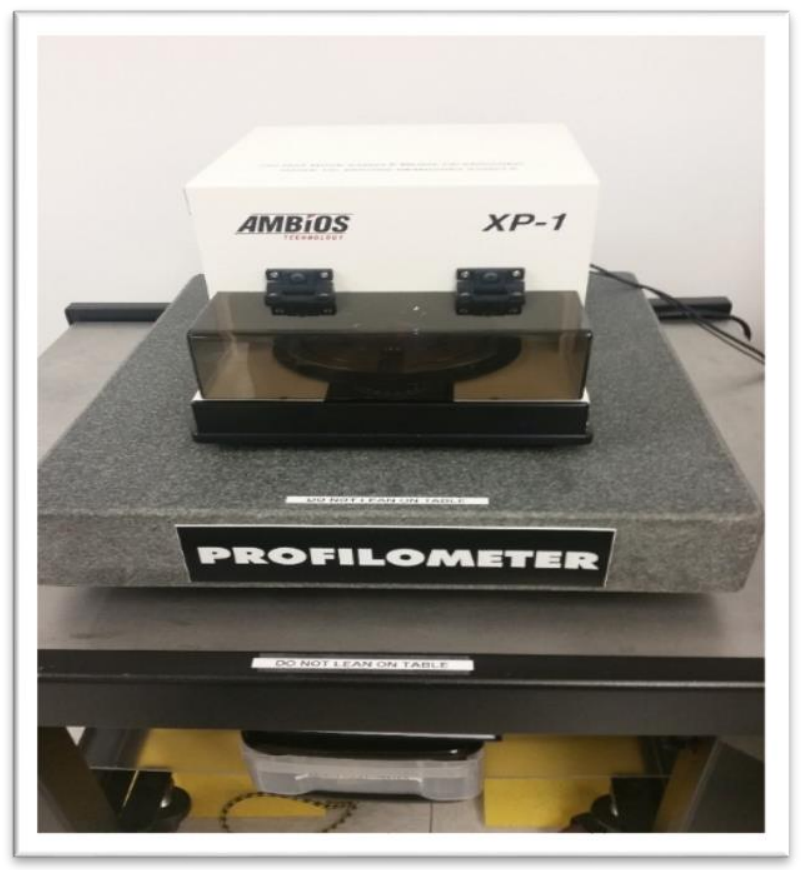

Figure 3.51 Profilometer Measurement Machine The antenna dies were placed one at a time in the machine.

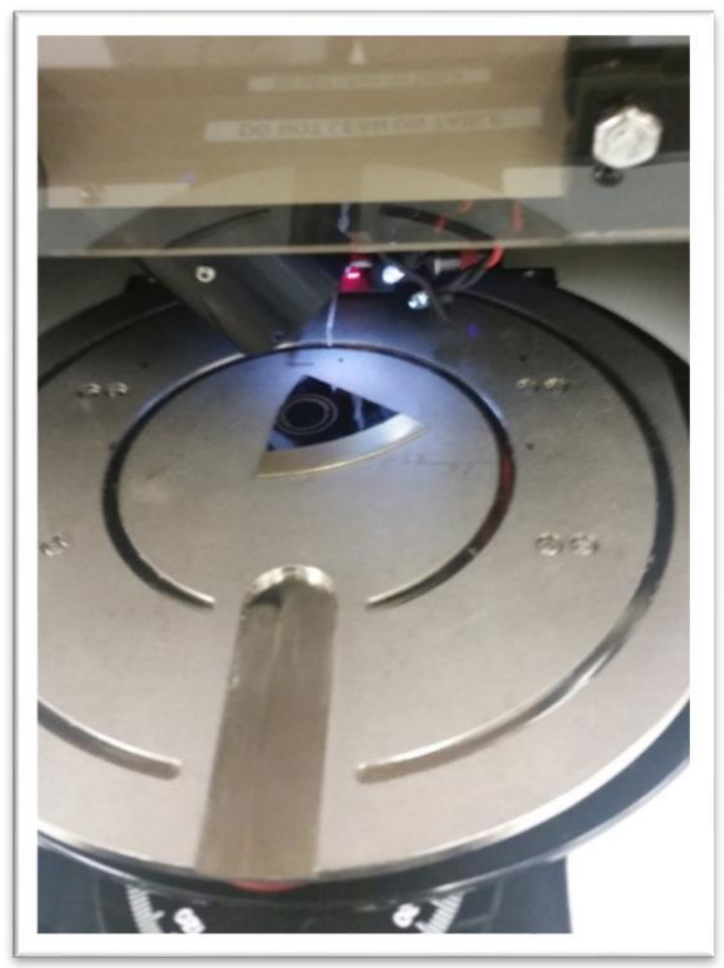

Figure 3.52 Inserted Sample in Profilometer 
The needle had descended enough to adjust the chuck for where the needle would engage on the substrate.

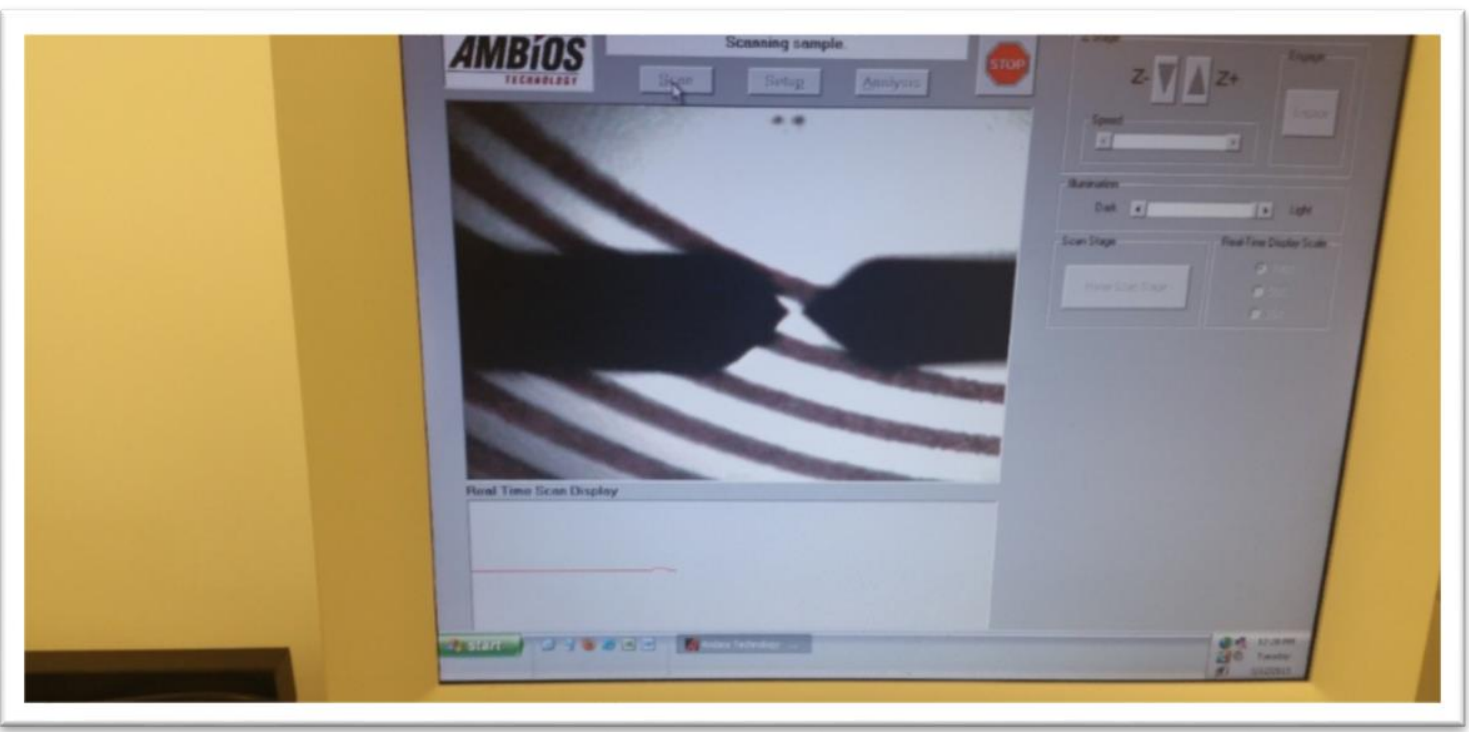

Figure 3.53 Scanning Surface of Sample

As the needle ran across the surface of the sample, the initial run scanned a

$5 \mathrm{~mm}$ distance on antenna 1. The results of the scan are shown in Figure 3.54 below. 


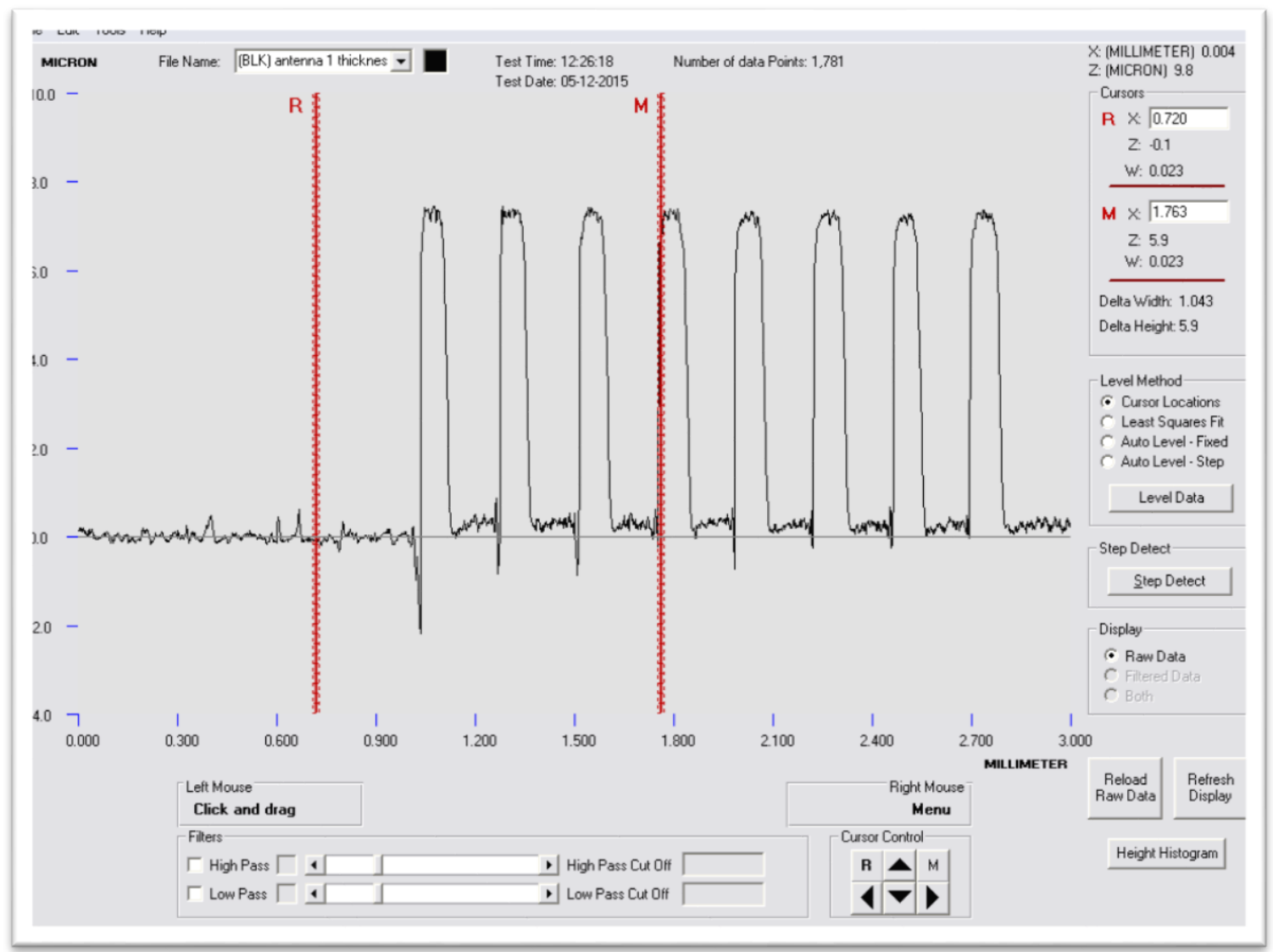

Figure 3.54 Results from Profilometer Scan Distance $5 \mathrm{~mm}$

The scanning distance was shortened to $3 \mathrm{~mm}$ and used for the antenna 2 and 3 design. The results are seen in Figure 3.55 (a) and (b). 


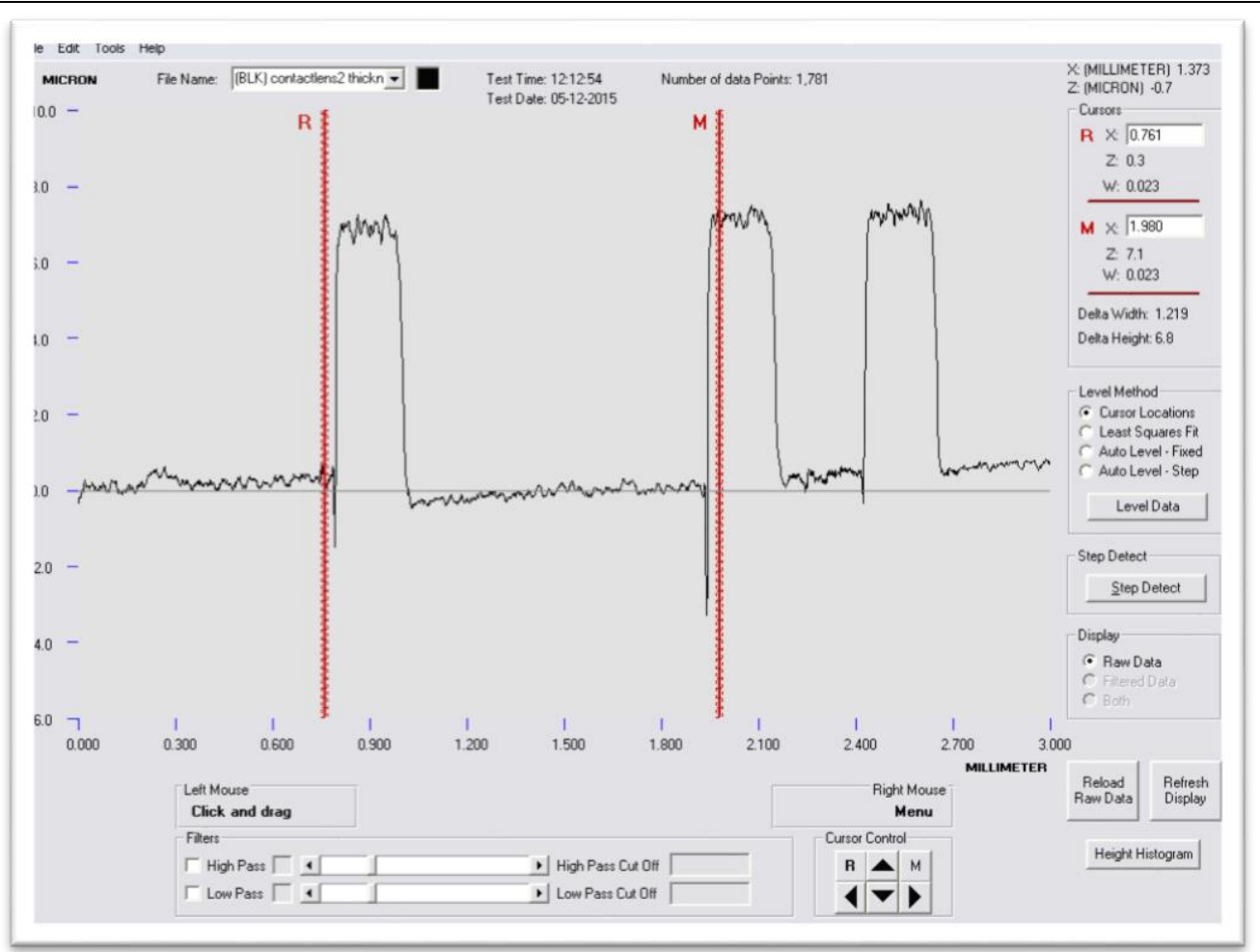

(a) Profilometer Data for Antenna 2

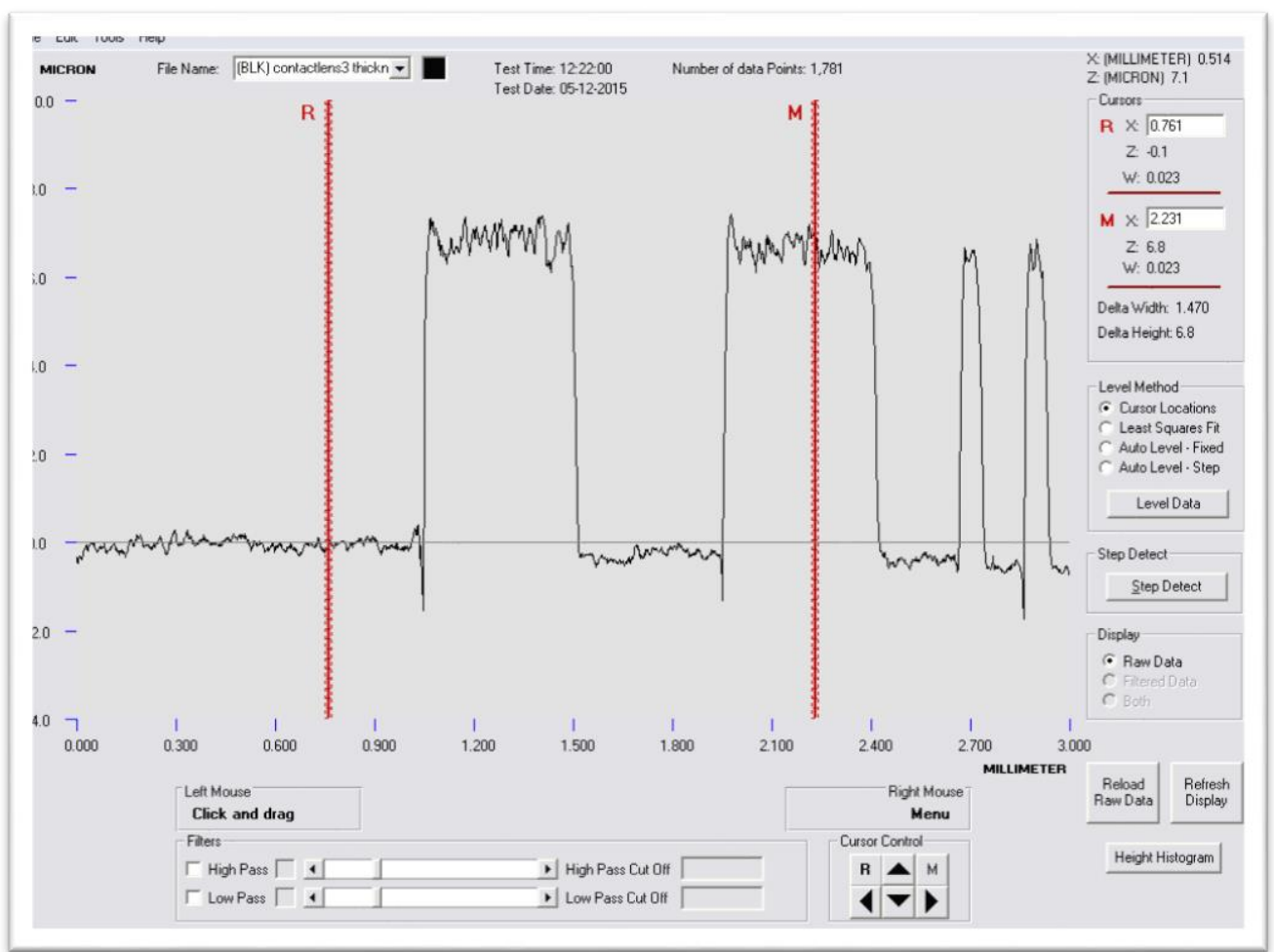

(b) Profilometer Data for Antenna 3

Figure 3.55 Results from Profilometer Scan Distance $3 \mathrm{~mm}$ 
From the results of the three antennas, it was found that the thickness of the traces were approximately 6.8um.

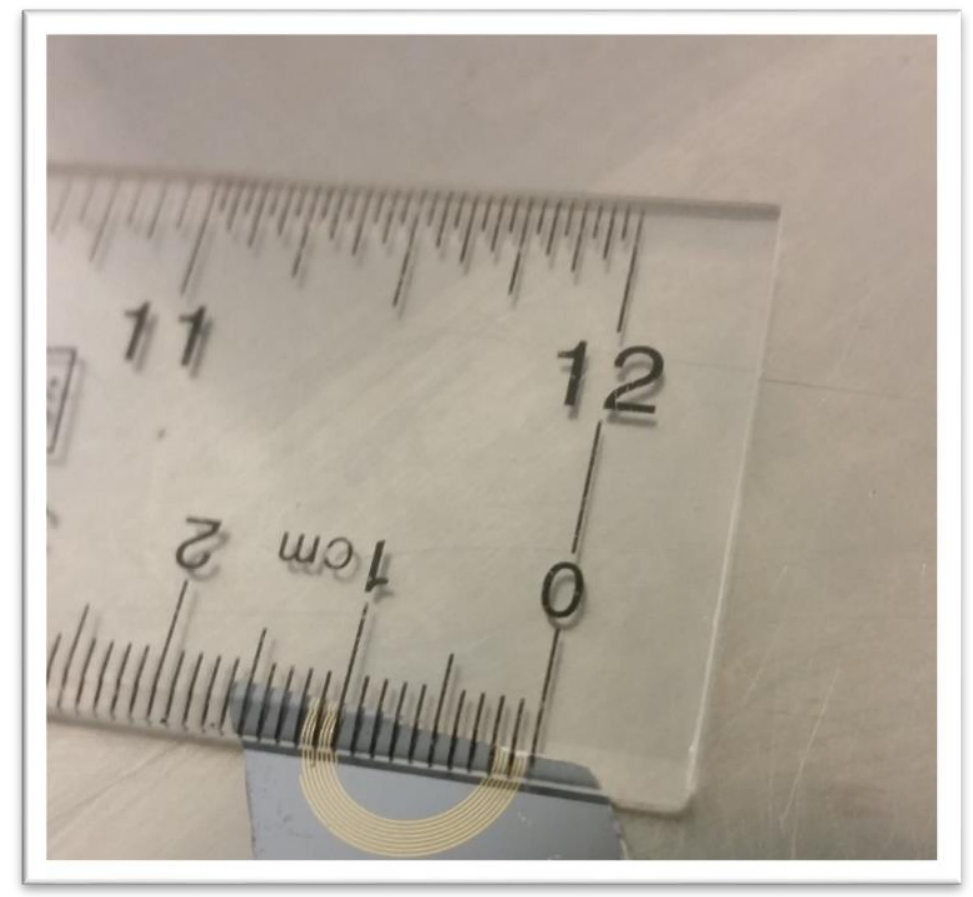

Figure 3.56 Example of antenna fabrication verification

The result of measuring many antennas, including the prototype lens, revealed that the microfabrication process is a remarkably accurate manufacturing method. Nearly all antennas were printed with an inner diameter of $8 \mathrm{~mm}$, within the tolerance threshold for the specification. Out of thirty-two fabricated antennas, only 3 had minor defects and 1 had a major defect.

\subsection{Antenna Testing}

The primary goal of radio frequency engineering performed during this research was to characterize the efficacy of the antenna as not only a receiver component of the active contact lens, but also as an inductive coil capable of charging the onboard battery. Due to the fact that all components are 
permanently affixed at the completion of manufacturing, proper inductance from the antenna is necessary to ensure sufficient power transfer is achieved as well as to ensure the lens does not become a onetime use product.

Three different antenna designs were considered when selecting an overall design for the project. The first design, shown in Figure 3.57, consisted of a continuous loop antenna of uniform trace width. This antenna was conceived under the assumption that the antenna could double as both an inductive coil and signal receiver. The second design, shown in Figure 3.58 on the next page, attempts to improve efficiency at $2.4 \mathrm{GHz}$ by separating the inductor coil from the antenna thereby decreasing resistance loss. The third design, shown in Figure 3.59 , is an adaptation of an IFA design that includes an inductive coil that is attached to the ground plane.

\begin{tabular}{|l|l|}
\hline $\mathrm{N}$ (turns) & 8 \\
\hline $\begin{array}{l}\mathrm{D}_{\mathrm{i}} \text { (inner } \\
\text { diameter) }\end{array}$ & $7885 \mathrm{um}$ \\
\hline $\begin{array}{l}\text { Do (outer } \\
\text { diameter) }\end{array}$ & $11630 \mathrm{um}$ \\
\hline $\begin{array}{l}\text { W (trace } \\
\text { width) }\end{array}$ & $117 \mathrm{um}$ \\
\hline $\begin{array}{l}\text { S (trace } \\
\text { spacing) }\end{array}$ & $117 \mathrm{um}$ \\
\hline
\end{tabular}

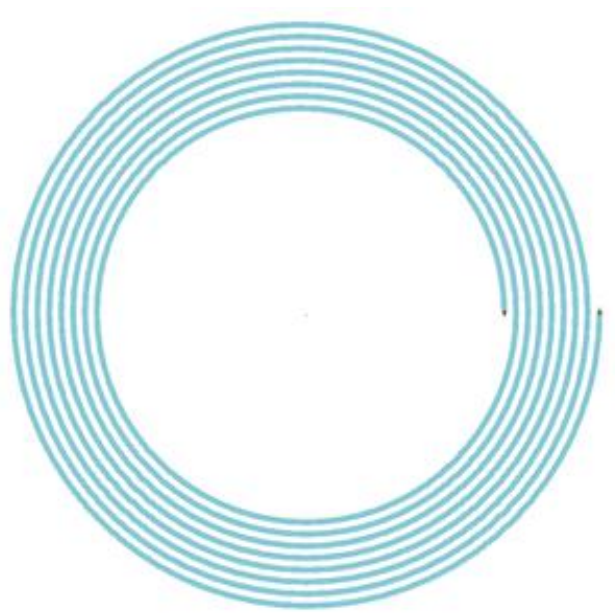

Figure 3.57 First Antenna Design, Consisting of One Solid Loop of Uniform Trace This was the first design with the assumption that we could use the inductive coil as the antenna. In order to fit the outer diameter size constraint, we were not able to use a $\lambda / 3$ design, so a $7 \lambda / 24$ circumference was used having an outer 
diameter of $11.63 \mathrm{~mm}$. This gave better efficiency than $\lambda / 4$ while remaining under $12 \mathrm{~mm}$ diameter. This coil had 8 turns with an inner diameter of $7.885 \mathrm{~mm}$, which is only $1.5 \%$ less than our inner diameter requirement. Out of our 3 designs, this had the highest inductance, but severely lacked power efficiency at $2.4 \mathrm{GHz}$. The simulation gave an antenna gain of $-22.3 \mathrm{~dB}$ at $2.4 \mathrm{GHz}$, which was the lowest of the designs. Using Harold A. Wheeler approximations for planar coils operating under $30 \mathrm{MHz}\left(L(\mu H)=\frac{D_{o}^{2} \times A^{2}}{30 \times(A-11) \times D_{i}}, A=\frac{D_{i}+N(W+S)}{2}\right)$, the inductance was calculated at $1.005 \mu \mathrm{H}$. This design was by far the best for inductive charging.

\begin{tabular}{|l|l|l|}
\hline Antenna Ring & Diameter & $11900 \mathrm{um}$ \\
\hline Antenna Ring & trace width & $500 \mathrm{um}$ \\
\hline Antenna Ring & trace spacing & $103.5 \mathrm{um}$ \\
\hline Inner coil & $\mathrm{N}$ (turns) & 1.605 \\
\hline Inner coil & $\mathrm{D}_{\text {i }}$ (inner diameter) & $8000 \mathrm{um}$ \\
\hline Inner coil & $\mathrm{D}_{\circ}$ (outer diameter) & $9262.7 \mathrm{um}$ \\
\hline Inner coil & W (trace width) & $250 \mathrm{um}$ \\
\hline Inner coil & S (trace spacing) & 250um \\
\hline
\end{tabular}

Figure 3.58 Second Antenna Design, Two Separate Loops of Uniform Trace Width This second design makes use of a large trace antenna outer loop and a 1.6 turn inner coupling coil to achieve a gain of $-14.5 \mathrm{~dB}$ at $2.4 \mathrm{GHz}$. This greatly improved gain sacrifices inductance of $20 \mathrm{nH}$, which was based on the minimal 
windings of the inner coil. This was able to almost double its power efficiency by doubling the trace width of the antenna coil.

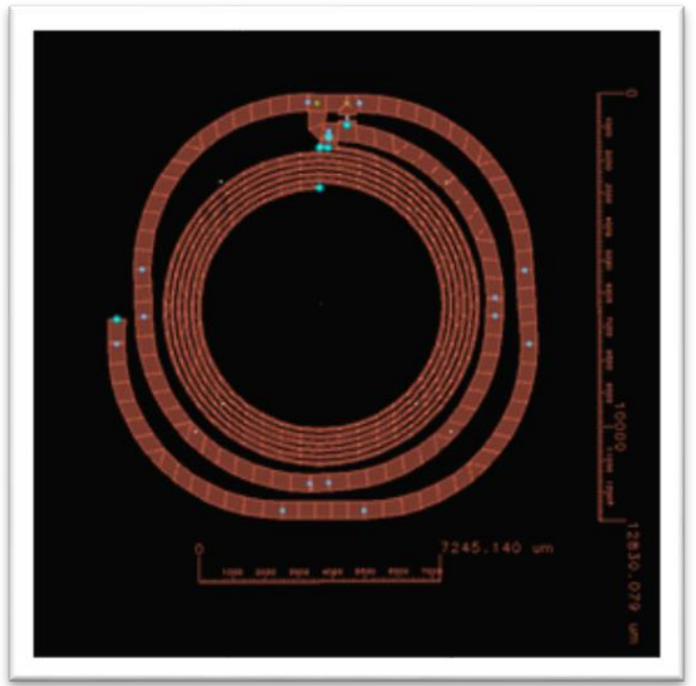

Figure 3.59 Third Antenna Design, Two Separate Loops of Non-Uniform Trace Width This dual loop design used an inside coil, even though they are not connected, coupled into the actual antenna and had a direct effect on the resonant frequency of the outside loop. The loop antenna had a small coupling loop (roughly $1 / 5$ of the size of the antenna), which is fed the signal instead of the larger loop. The thicker loop is the antenna. The outer diameter is about $1.3 \mathrm{~cm}$ and the inner diameter is about $7.2 \mathrm{~mm}$. Efficiency was improved by using a thicker trace for the antenna compared to sticking to a uniform trace width. After all three antenna were simulated, it was determined that regardless of substrate and size, only the dual loop antennas would be appropriate for achieving the desired functionality. Figures $3.60,3.61$, and 3.62 below present the results from these simulation. 


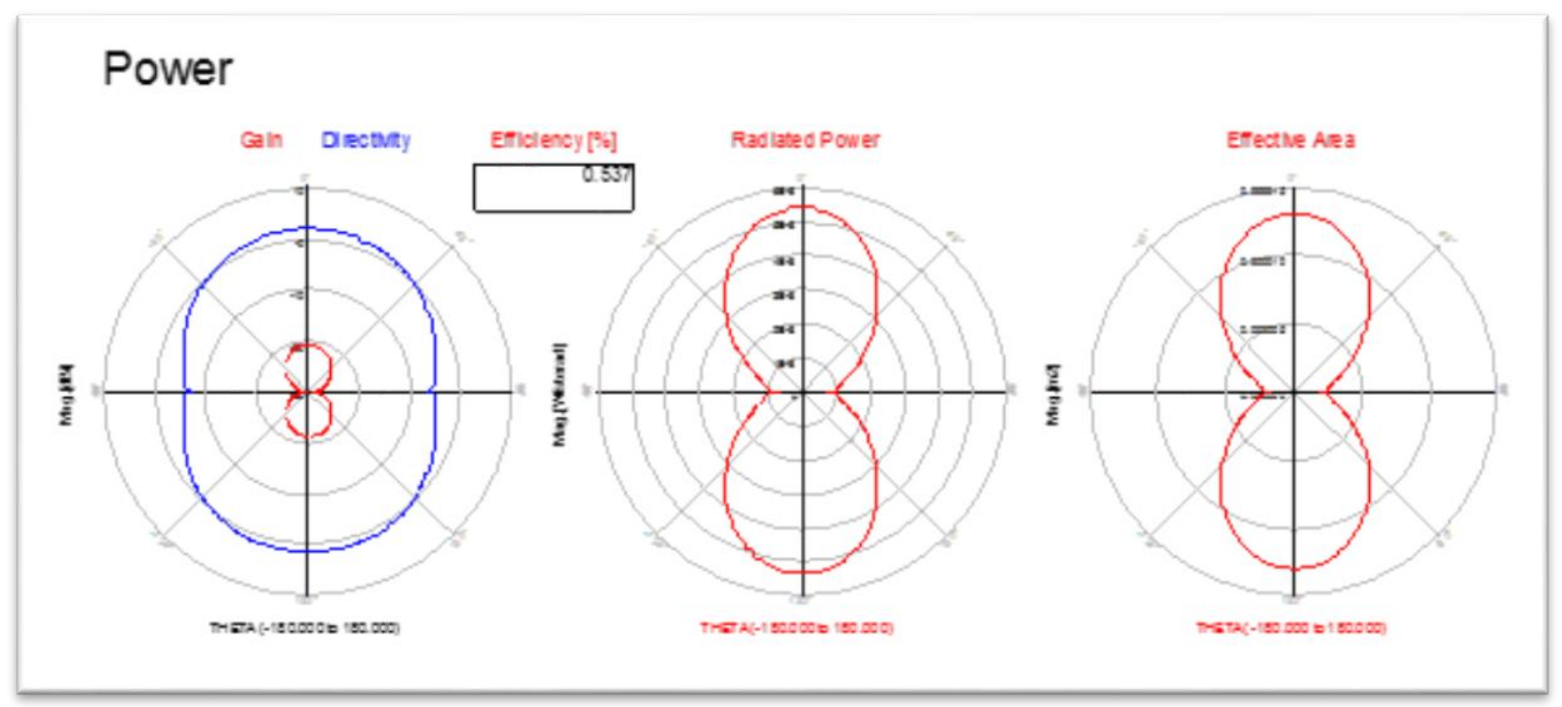

Figure 3.60 Antenna design 1 efficiency and power results

Immediately from Figure 3.60 above we see that antenna design 1 will not be a sufficient platform in providing wireless charging. The efficiency, which sits a $0.537 \%$, is too low to sufficiently facilitate power transfer.

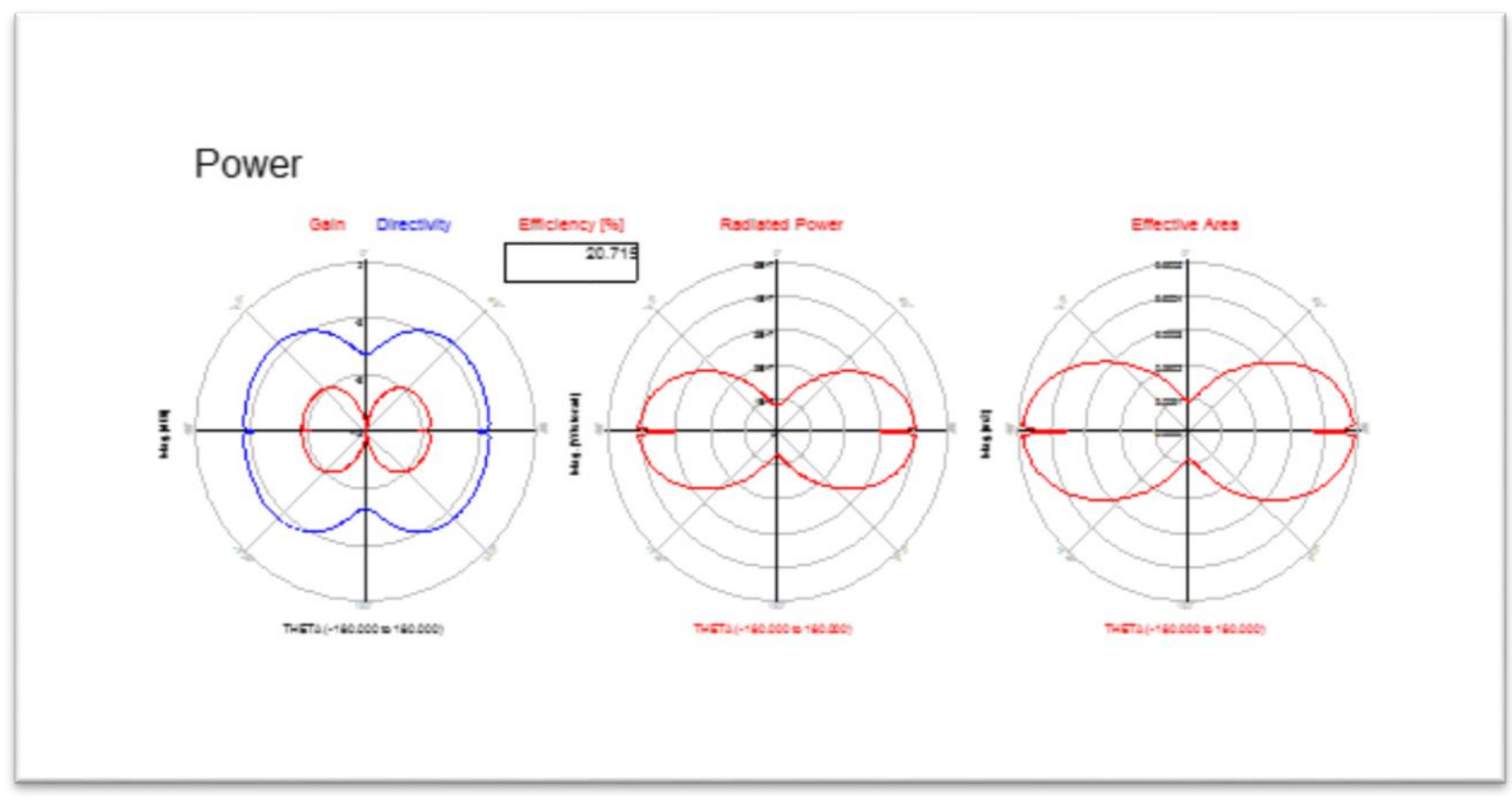

Figure 3.61 Antenna design 2 efficiency and power results

Antenna design 2, unlike design 1, displays much more promise as a platform for wireless charging. With an efficiency at $20.715 \%$, the design is more than 
viable for facilitating wireless power transfer.

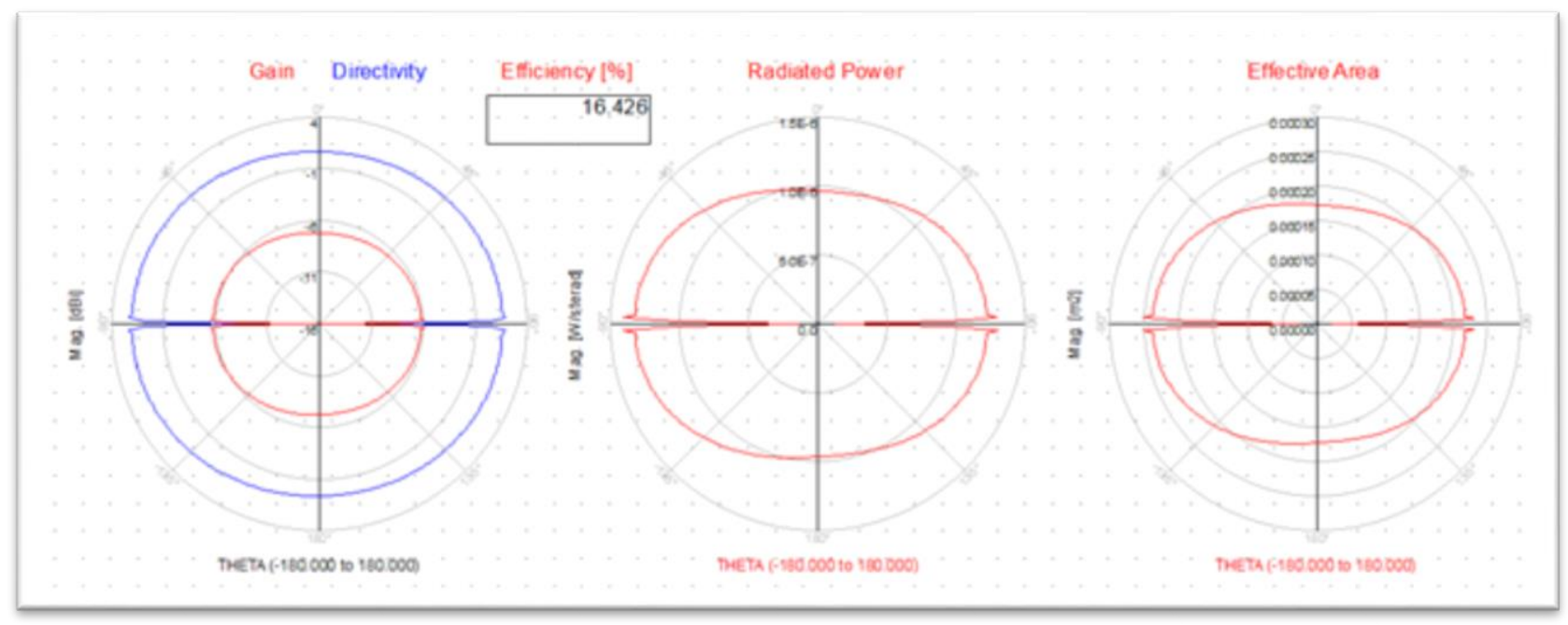

Figure 3.62 Antenna design 3 efficiency and power results

Antenna design 3 follows closely in line with design 2, albeit with a lower efficiency. Ideally, all the antenna designs will be able to receive signals regardless of efficiency. To meet our design requirements, however, it was imperative to choose a design that best suited the wireless charging platform. While antenna design 2 represents the highest efficiency, it has a much lower effective area, making it less suitable for wider range of communication distances. Geometrically, antenna design 2 also interferes with our clear vision requirement, just barely clearing the maximal pupil dilation diameter requirement. As such, it was decided that antenna design 3 would be the best option to pursue towards the final design.

The three printed antenna designs and micro-fabricated antenna designs were tested for performance and far-field characterization using the following microwave lab equipment and a test antenna. This will compare the difference 
between the simulation and fabricated antenna response as well as determine whether printing or microfabrication process is better.

Equipment: $17 \mathrm{~cm}$ reference antenna/transmitter

\section{Anritsu MS4622B} Network Analyzer

CXA Signal Analyzer
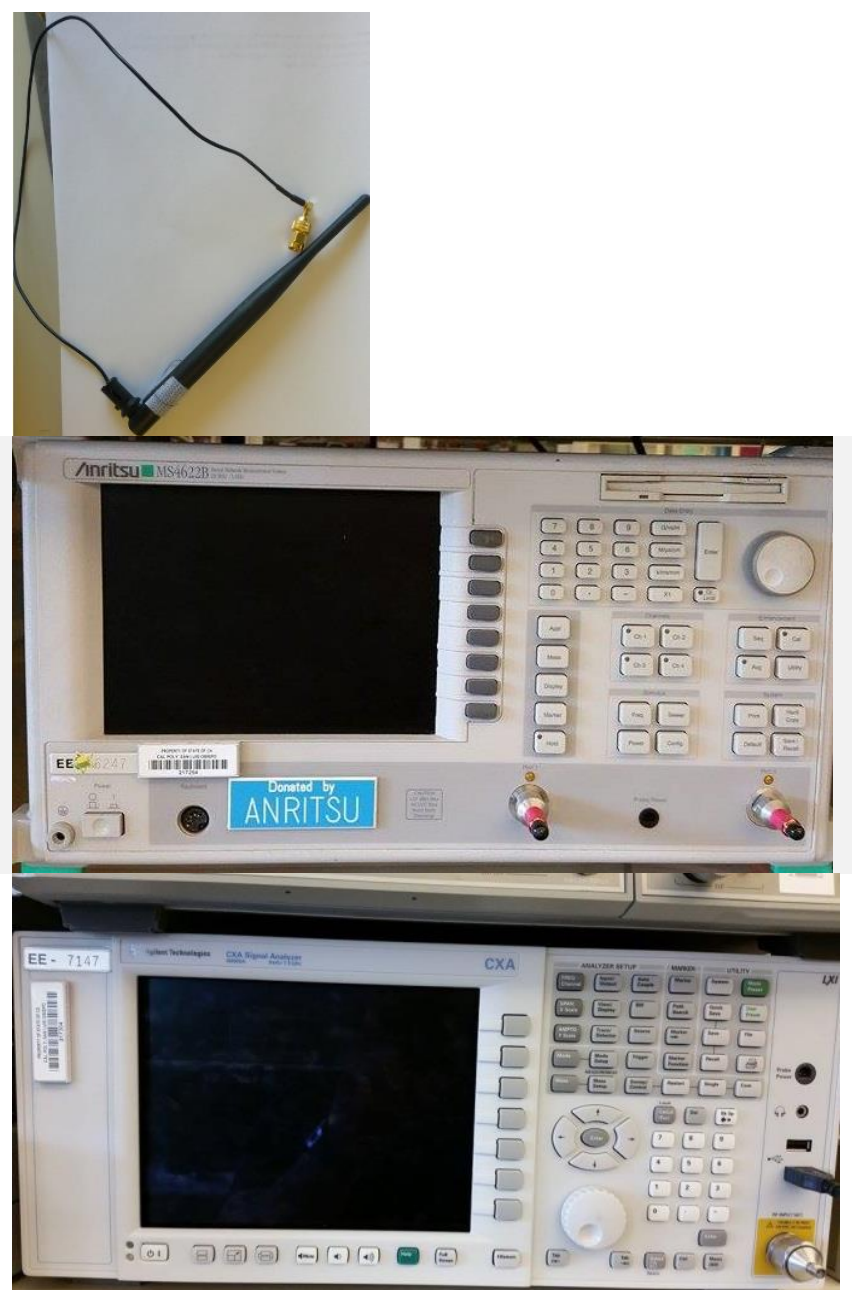

\section{Synthesized Sweep Generator}

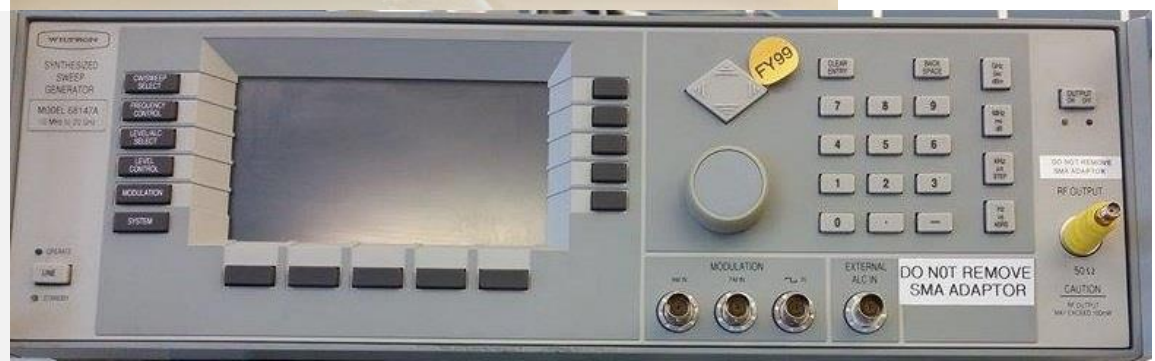




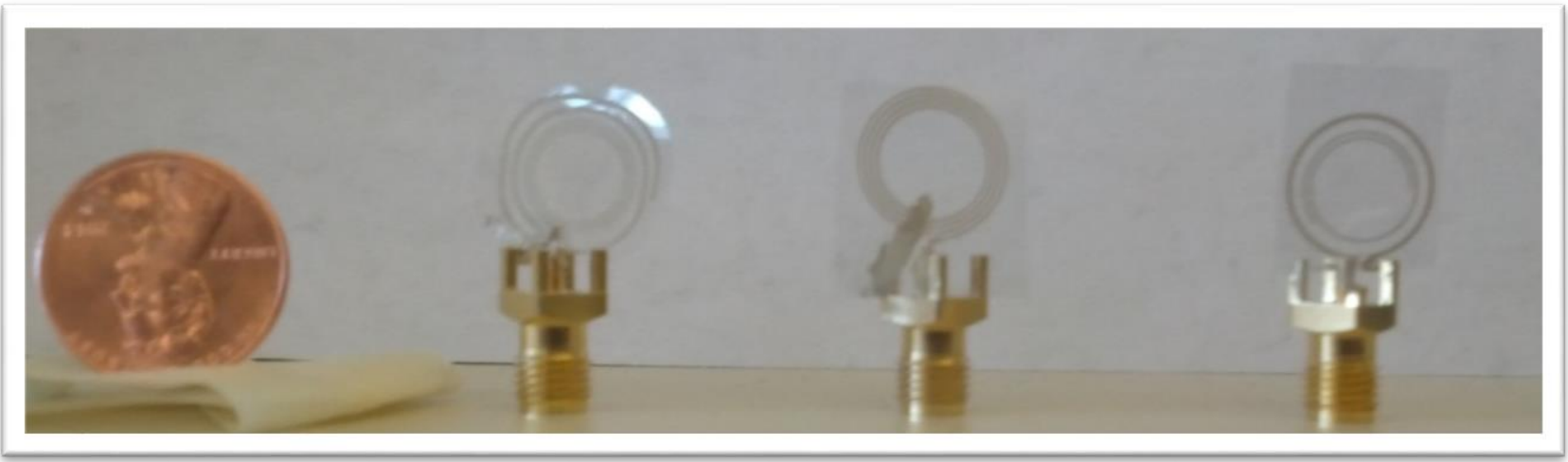

Figure 3.63 Antennas under testing. AUT1, AUT2, AUT3 from Left to Right

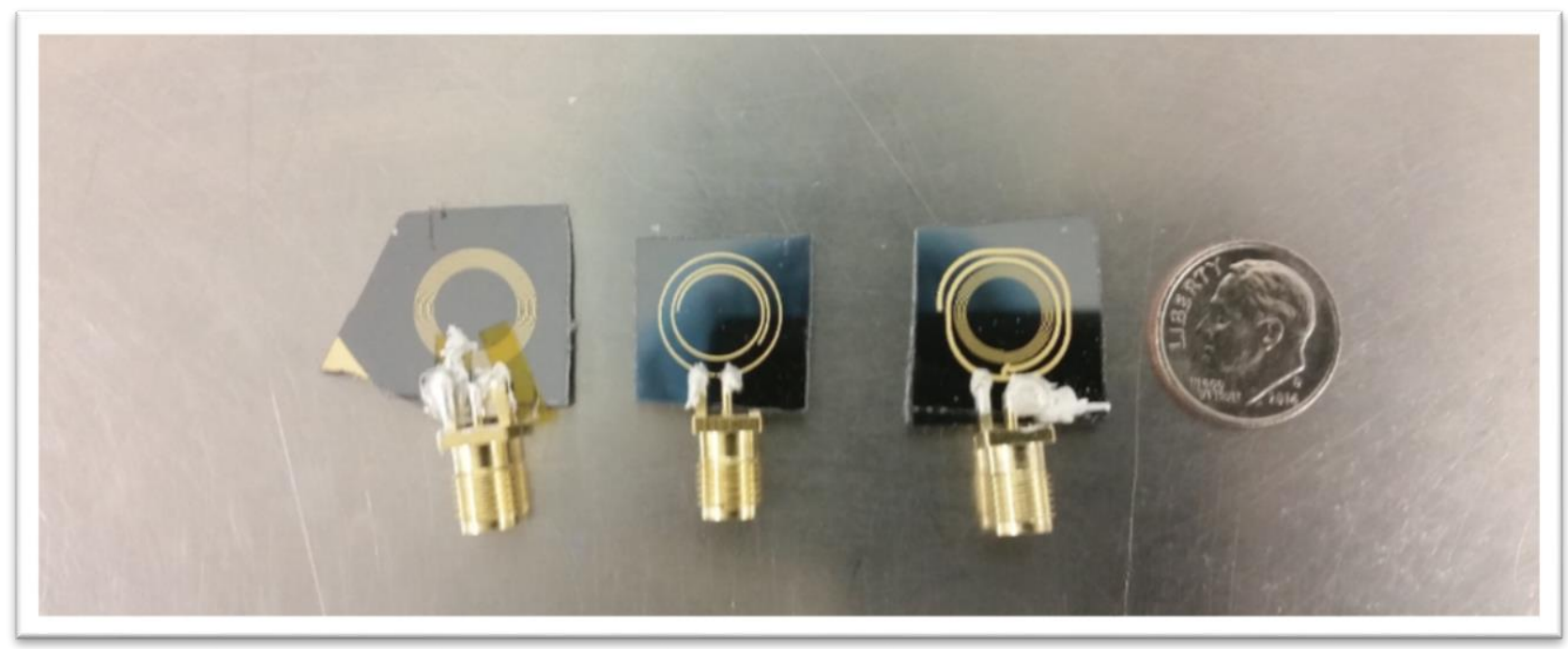

Figure 3.64 Antennas under testing. AUT4, AUT5, AUT6 from Left to Right The preceding subsections list the results of the following test parameters for AUT1, AUT2, and AUT3: Gain, Impedance and Bandwidth, Polarization and Beam width, Radiation pattern (magnitude) and efficiency, and Radiation Resistance

\subsubsection{Gain Test}

The transmitter has an unknown gain, which can be determined by testing the received power of each AUT and reference antenna. This test will also determine the gains for all 3 printed AUTs. Using a set of four conservation of 
power equations and four unknowns, the gain of each antenna can be calculated.

$$
\begin{array}{lll}
P_{R_{r e f-1}}=P_{T X}+G_{r e f}+G_{1}-P F & \text { (3.8) } & P_{R_{r e f-2}}=P_{T X}+G_{r e f}+G_{2}-P F \\
P_{R_{r e f-3}}=P_{T X}+G_{r e f}+G_{3}-P F & \text { (3.10) } & P_{R_{1-2}}=P_{T X}+G_{1}+G_{2}-P F
\end{array}
$$

In order to accurately test the antennas under far-field condition, the far-field range must first be determined which is

$$
R \geq 2 D^{2} / \lambda
$$

where $D$ is the length of our source reference antenna and $\lambda$ is the operating wavelength, $12.5 \mathrm{~cm}$.

$$
R \geq 2(17 \mathrm{~cm})^{2} / 12.5 \mathrm{~cm}=46.24 \mathrm{~cm}
$$

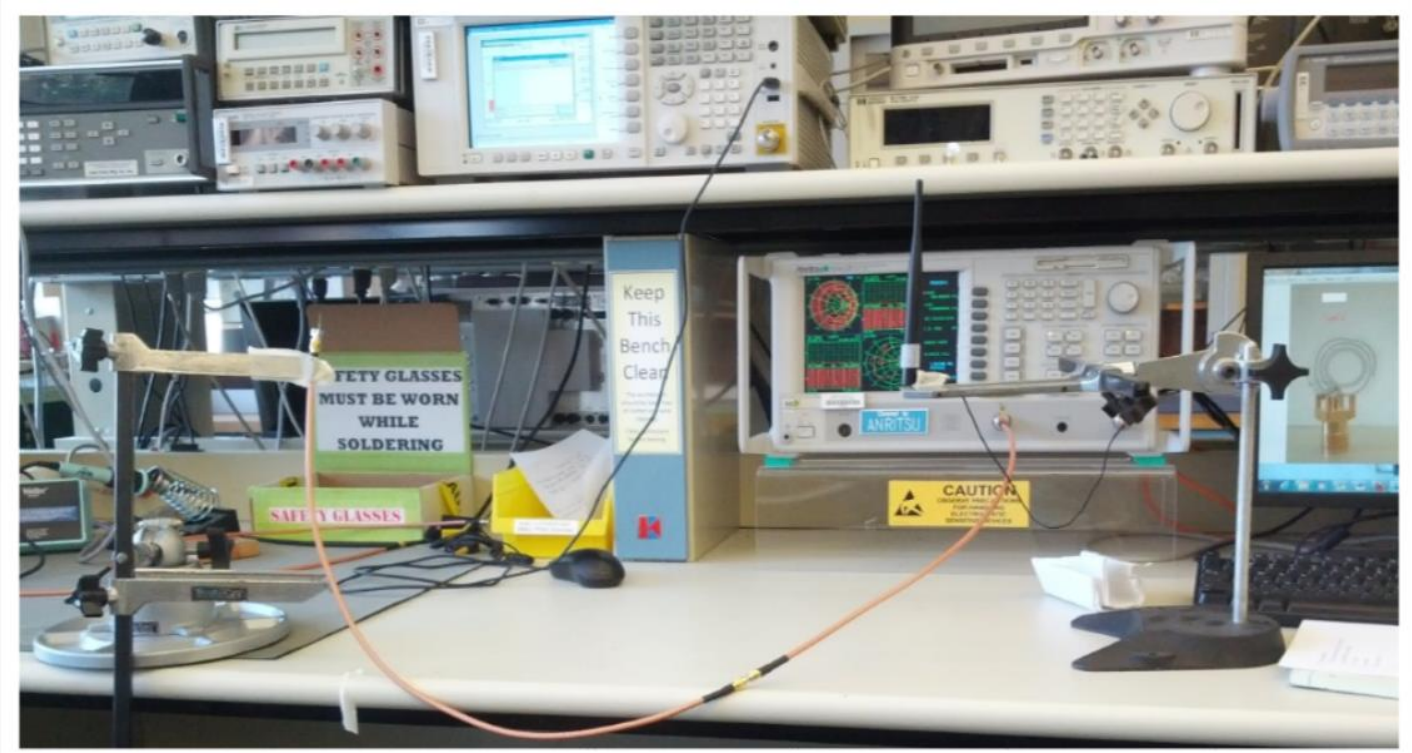

Figure 3.65 Antenna Gain Test Setup for 2.4GHz Far-Field Range of 50cm A distance of $50 \mathrm{~cm}$ was used for the entirety of the tests. At $50 \mathrm{~cm}$, the path loss at 2.4 $\mathrm{GHz}$ was calculated with free space approximation.

$$
P F(d B)=10 \log \left(\frac{4 \pi d}{\lambda}\right)^{2}=34 d B
$$


The power transmission test setup included the reference antenna mounted $50 \mathrm{~cm}$ away from the test antennas and transmitting $1 \mathrm{~dB}$ from the frequency generator. The test antennas were mounted on the SMA adapter for easy swapping.

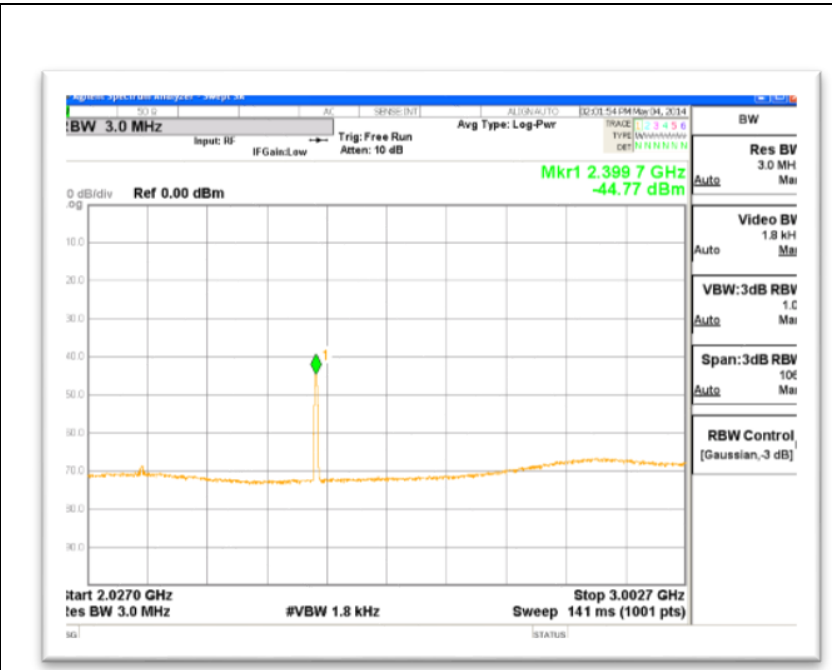

(a) Reference antenna transmitting $1 \mathrm{~dB}$ to Antenna 1 at $50 \mathrm{~cm}$

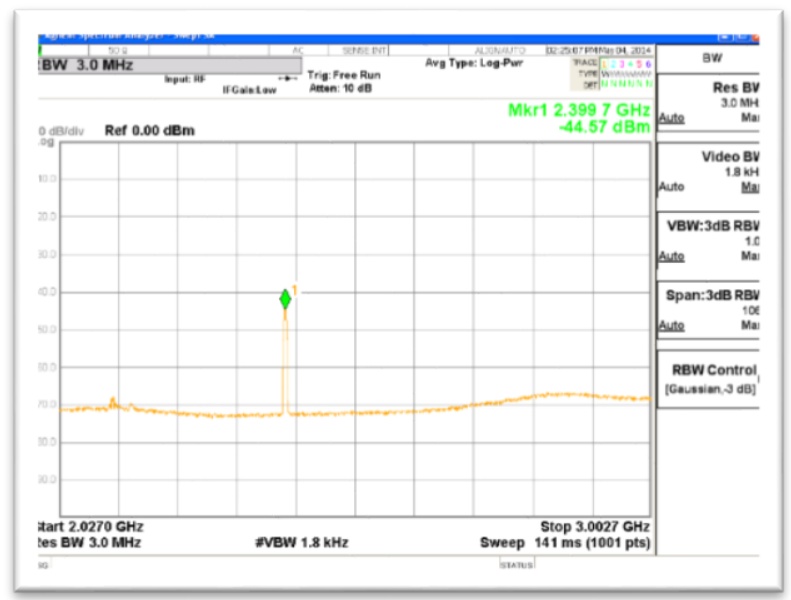

(c) Reference antenna transmitting $1 \mathrm{~dB}$ to Antenna 3 at $50 \mathrm{~cm}$

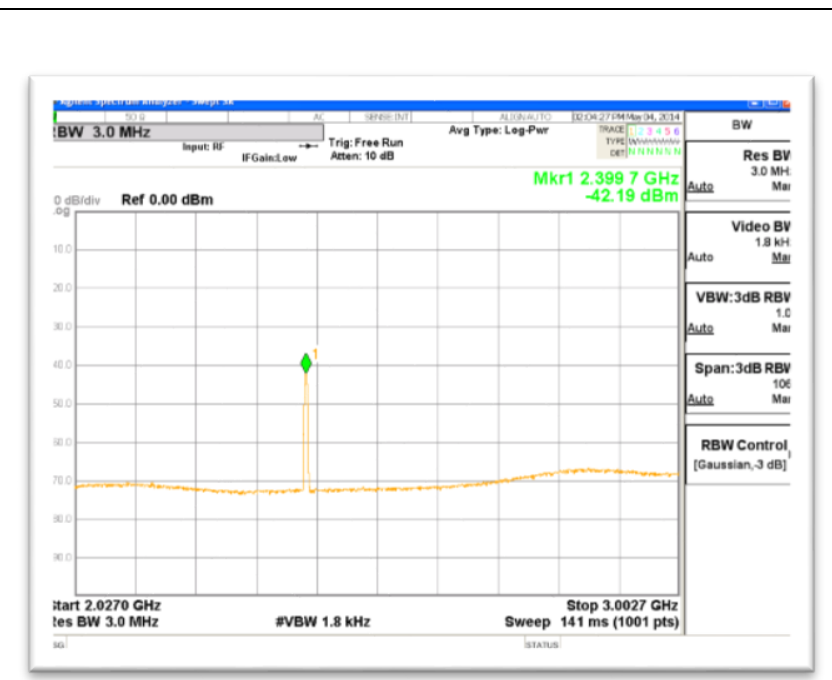

(b) Reference antenna transmitting $1 \mathrm{~dB}$ to Antenna 2 at $50 \mathrm{~cm}$

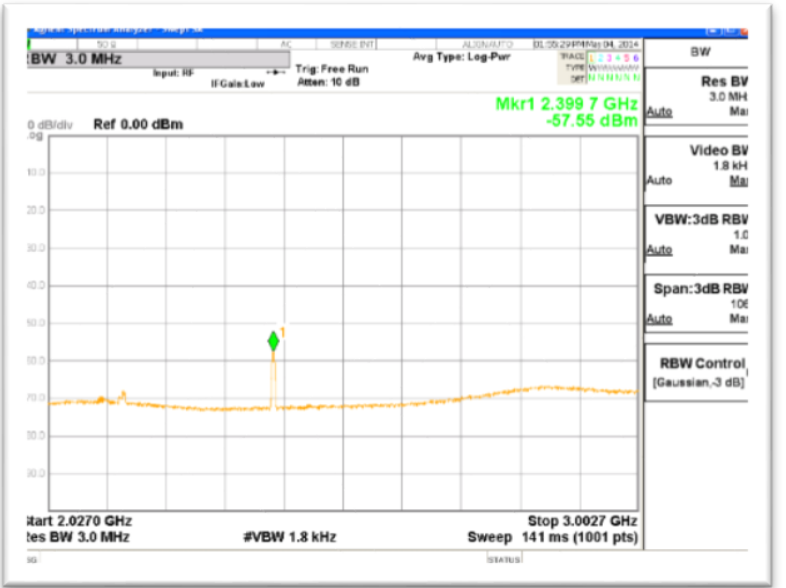

(d) Antenna 2 transmitting $1 \mathrm{~dB}$ to Antenna 1 at $50 \mathrm{~cm}$

Figure 3.66 Data from Spectrum Analyzer

Solving the conservation of power equations with the received power data gave us the gains for each antenna. 
$-44.77=1+G_{r e f}+G_{1}-34 \quad-42.19=1+G_{r e f}+G_{2}-34$

$-44.57=1+G_{r e f}+G_{3}-34 \quad-57.55=1+G_{1}+G_{2}-34$

$G_{r e f}=1.7950 \mathrm{dBi}, G_{1}=-13.565 \mathrm{dBi}, G_{2}=-10.985 \mathrm{dBi}, G_{3}=-13.365 \mathrm{dBi}$

3.5.2 Impedance and Bandwidth Test

Using the Anritsu MS4622B Network Analyzer, the first iteration antennas'

impedance and bandwidth were measured from their reflection coefficient $(\Gamma)$,

$S_{11}$ parameter.

\begin{tabular}{|c|c|c|c|}
\hline Parameter & AUT1 & AUT2 & AUT3 \\
\hline S11 $(\Gamma)[\mathrm{dB}]$ & -5.722 & -2.346 & -3.476 \\
\hline Power-ratio & 0.2678 & 0.5826 & 0.4492 \\
\hline VSWR [dB] & 1.73 & 3.79 & 2.63 \\
\hline $\begin{array}{c}\text { Reflected } \\
\text { Power [\%] }\end{array}$ & 7.2 & 33.9 & 20.2 \\
\hline Impedance & $\begin{array}{l}19.744- \\
\text { j22.993 }\end{array}$ & $\begin{array}{l}13.003- \\
\text { j49.497 }\end{array}$ & $194.772+\mathrm{j} 281.877$ \\
\hline
\end{tabular}

Table 2 Antenna Parameters Calculated from Reflection Coefficient

Sample calculations:

Power - ratio $=10^{\frac{\Gamma}{10}} \quad$ For AUT1, $10^{\frac{-5.722}{10}}=0.2678$

VSWR $=\frac{1+\text { power-ratio }}{1-\text { power-ratio }} \quad$ For AUT1, VSWR $=\frac{1+0.2678}{1-0.2678}=1.73$

Reflected Power $(\%)=100 *|\Gamma|^{2} \quad$ For AUT1, Refl. Power $=100 \%{ }^{*} 0.2678^{2}$

$=7.2 \%$

The impedance response and magnitude response of AUT1 are shown below in

Figure 3.67 (a) and (b). 


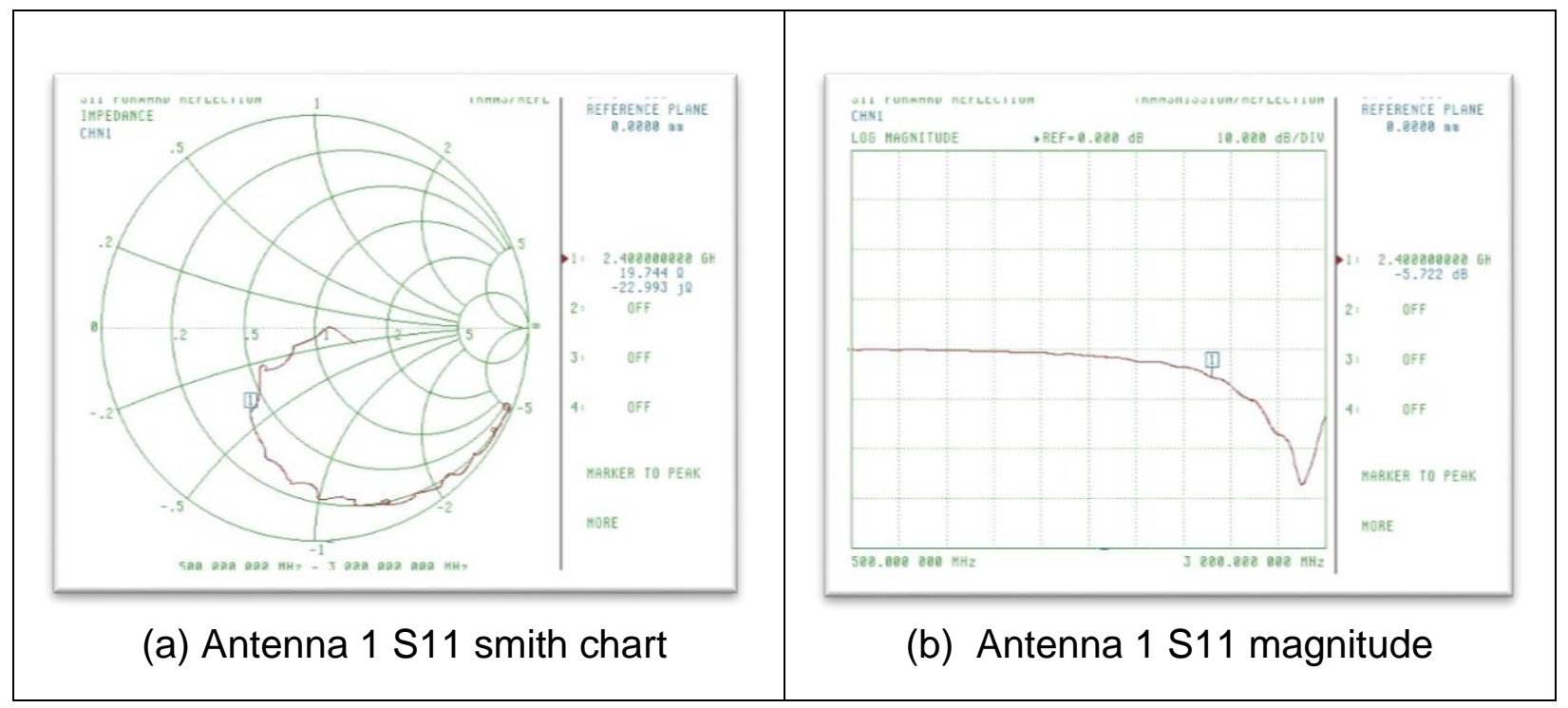

Figure 3.67 Impedance and Magnitude Response for AUT1

The impedance value for AUT1 was 19.744-j22.993 and an $\mathrm{S}_{11}$ of $-5.722 \mathrm{~dB}$ at

2.4 $\mathrm{GHz}$. The reactance for the antenna at $2.4 \mathrm{GHz}$ frequency is capacitance which will create power loss by hinder maximum power transfer from the impedance mismatch resulting in a smaller powered signal being received by the IC. The desired dip in the $S_{11}$ magnitude response is at approximately 2.8 $\mathrm{GHz}$ indicating that the total length of the antenna is not long enough which is why it is responding better at a shorter wavelength.

The impedance response and magnitude response of AUT2 are shown below in Figure 3.68 (a) and (b). 


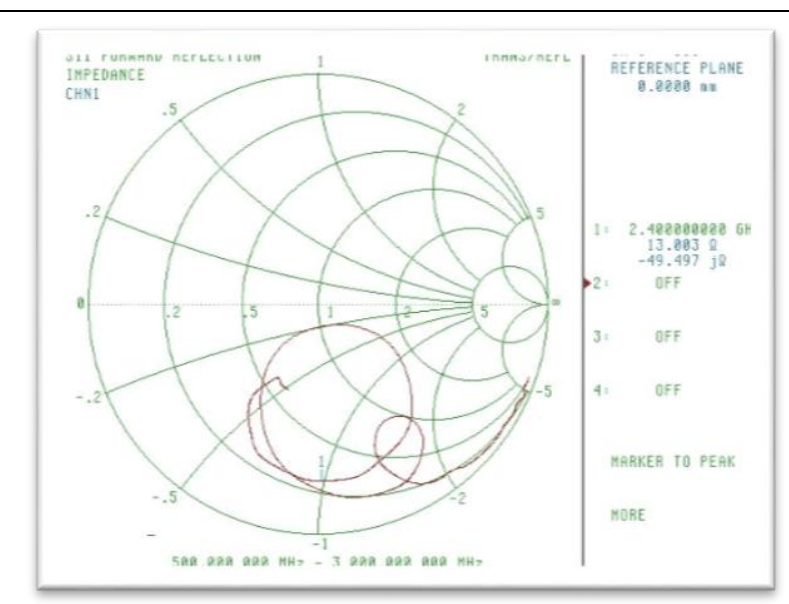

(a) Antenna 2 S11 smith chart

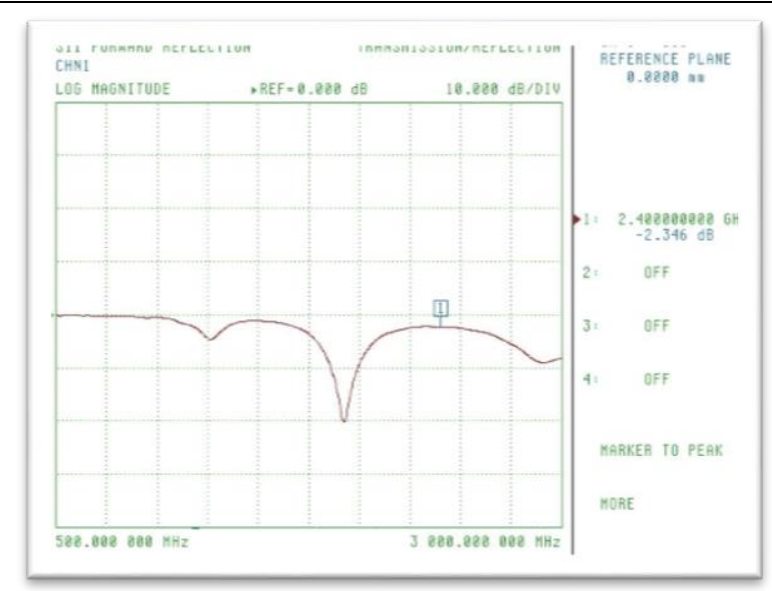

(b) Antenna 2 S11 magnitude

Figure 3.68 Impedance and Magnitude Response for AUT2

The impedance value for AUT2 was 13.003-j49.497 and an S11 of -2.346dB at

2.4 $\mathrm{GHz}$. The reactance for this antenna at $2.4 \mathrm{GHz}$ frequency has

approximately twice the capacitance resulting in an even smaller $\mathbf{S 1 1}$ relative to AUT1. The desired $S_{11}$ magnitude response is at approximately $1.9 \mathrm{GHz}$ indicating that the total length of the antenna is too long and can be shortened to shift the response to a higher frequency.

The impedance response and magnitude response of AUT3 are shown below in Figure 3.69 (a) and (b). 


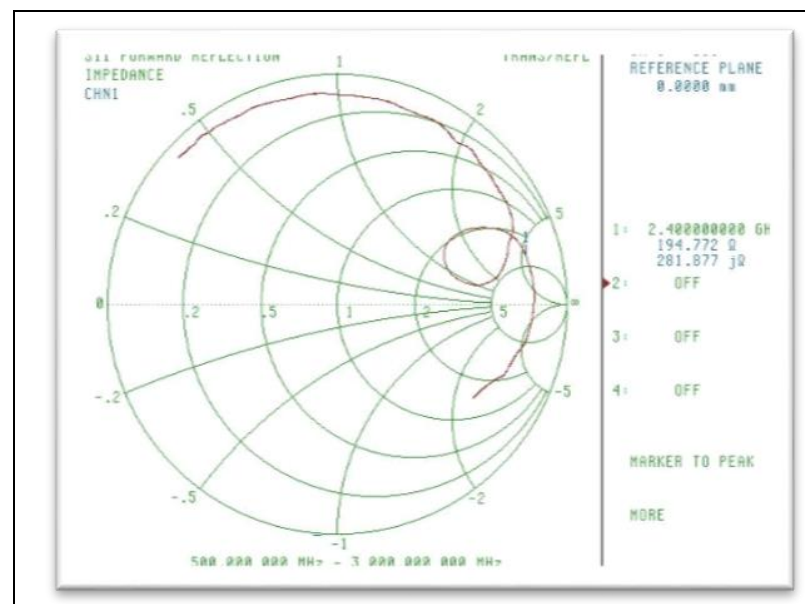

(a) Antenna 3 S11 smith chart

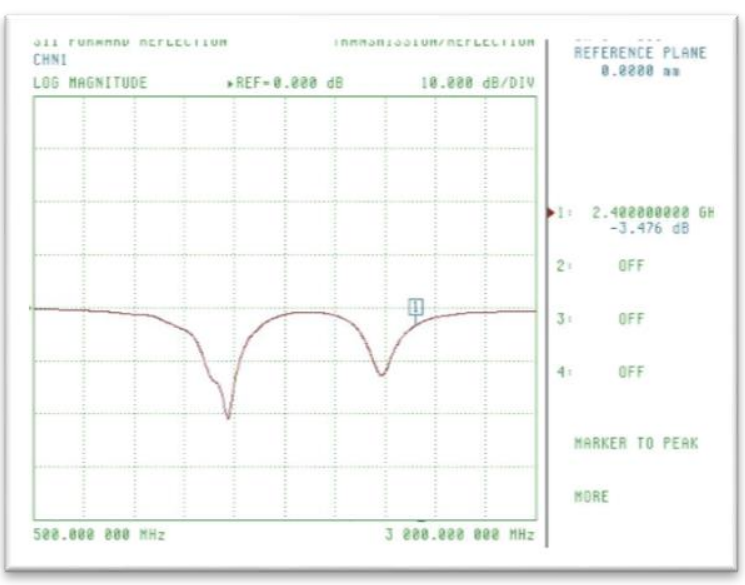

(b) Antenna 3 S11 magnitude

Figure 3.69 Impedance and Magnitude Response for AUT3

The impedance value for AUT3 was $194.772+\mathrm{j} 281.877$ and an $\mathrm{S}_{11}$ of $-3.476 \mathrm{~dB}$ at $2.4 \mathrm{GHz}$. The reactance for this antenna at $2.4 \mathrm{GHz}$ frequency has a high inductance value. The desired $S_{11}$ magnitude response is at approximately 2.2 $\mathrm{GHz}$ indicating that the total length of the stub on the antenna is too long and can be shortened to shift the response to a higher frequency. A second iteration was made on AUT1 and AUT3 because the response was not at all similar to simulation. This may have been due to the dielectric constant of the substrate not being similar the value given in simulation. During the second test the following results are shown in Figure 3.70.

The magnitude response of the $S_{11}$ looked similar to simulation and the desired -8.219 $\mathrm{dB}$ response was at frequency $2.2 \mathrm{GHz}$ instead of the designed $2.4 \mathrm{GHz}$. Additionally, the actual $S_{11}$ was higher than simulated results by $4 \mathrm{~dB}$. These discrepancies may have been caused by parasitic capacitances between the ink and PET substrate. In addition, cured ink may have been more resistive than anticipated in simulations. 


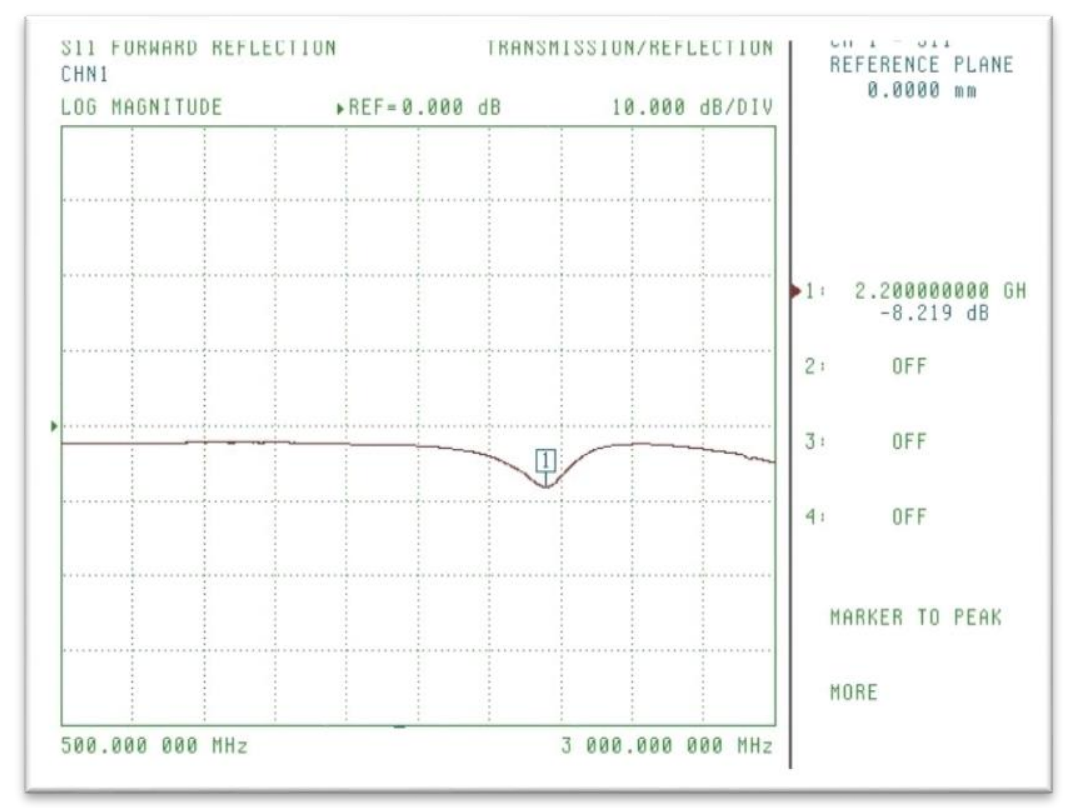

Figure 3.70 S11 of Second Iteration Printed IFA

Since the length of the stub on the antenna determines its operating frequency, it was determined that the length of the stub was too long based of $S_{11}$. The antenna needed to be tuned. This was done by scraping off the ink at the tip of the stub while having it connected to a Vector Network Analyzer (VNA). The frequency shift was closely monitored as the stub was carefully shortened until it reach $2.4 \mathrm{GHz}$ as shown in Figure 3.71. Figure 3.71 shows a reflection coefficient of $-9.582 \mathrm{~dB}$ at $2.4 \mathrm{GHz}$. 


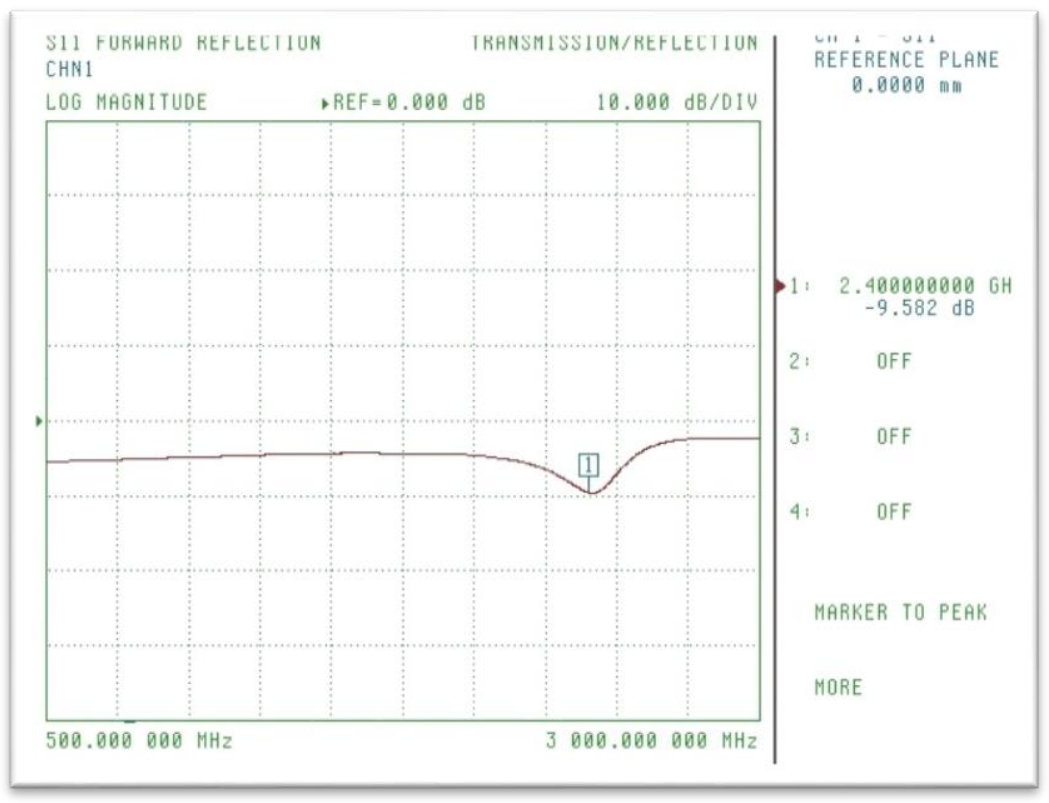

Figure 3.71 S11 of AUT3 Printed IFA Design after Tuning

Figure 3.72 shows the magnitude response of second iteration AUT1 with a reflection coefficient of $-4.446 \mathrm{~dB}$ at $2.38 \mathrm{GHz}$.

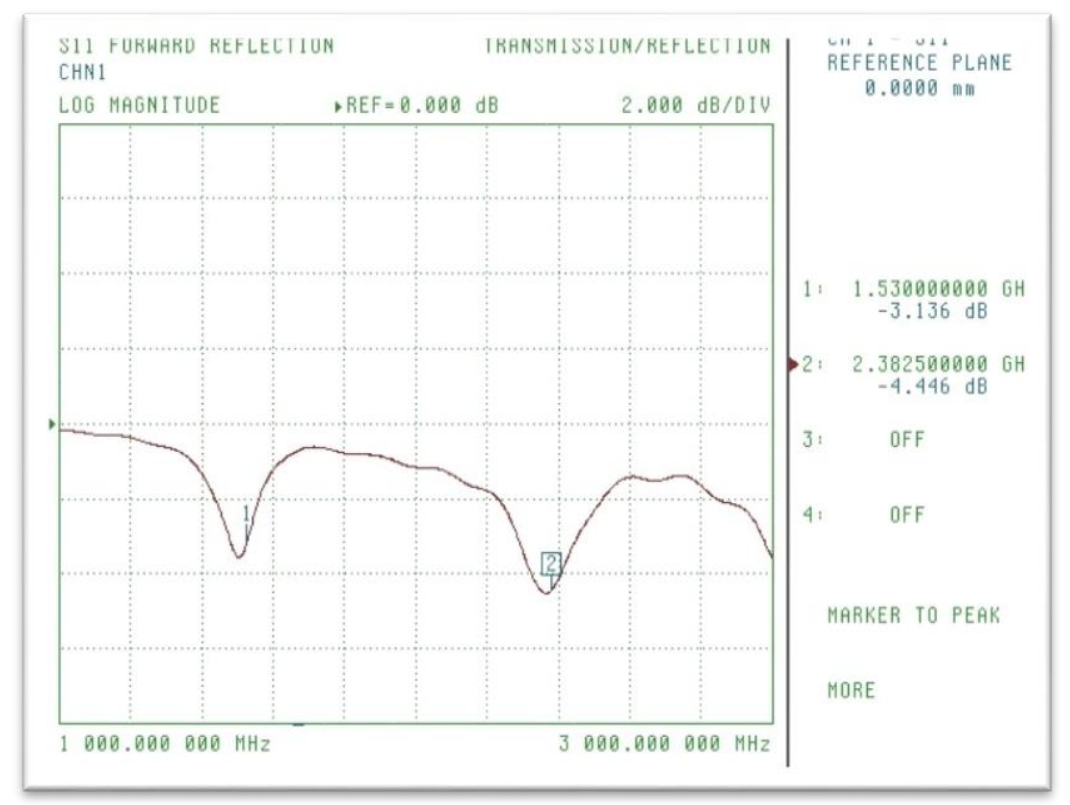

Figure 3.72 S11 of Second Iteration AUT1 Printed Coil Design 
Figure 3.73 and 3.74 show an inductance of $273.4 \mathrm{nH}$ ( $\mathrm{Q}$ factor of 0.269 ) and $268.2 \mathrm{nH}$ ( $Q$ factor of 0.98 ) for the IFA and Coil designs respectively. The trace resistances were higher than expected which resulted in a low $Q$ factors.

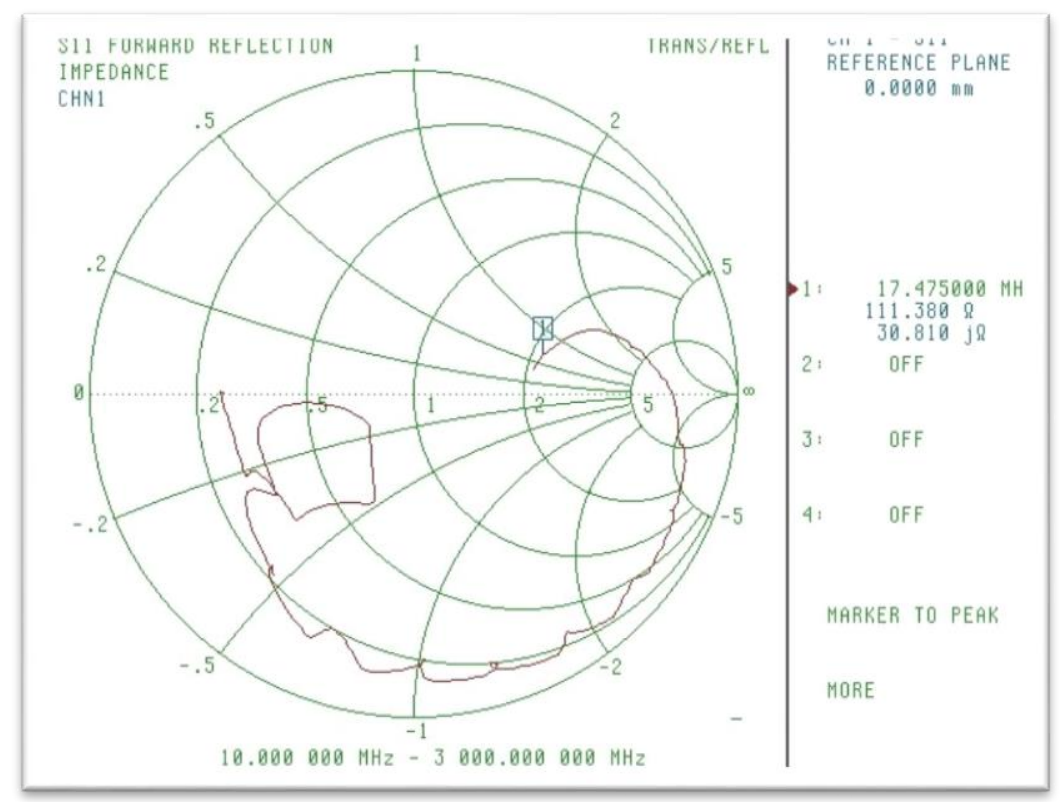

Figure 3.73 IFA Coil Inductance

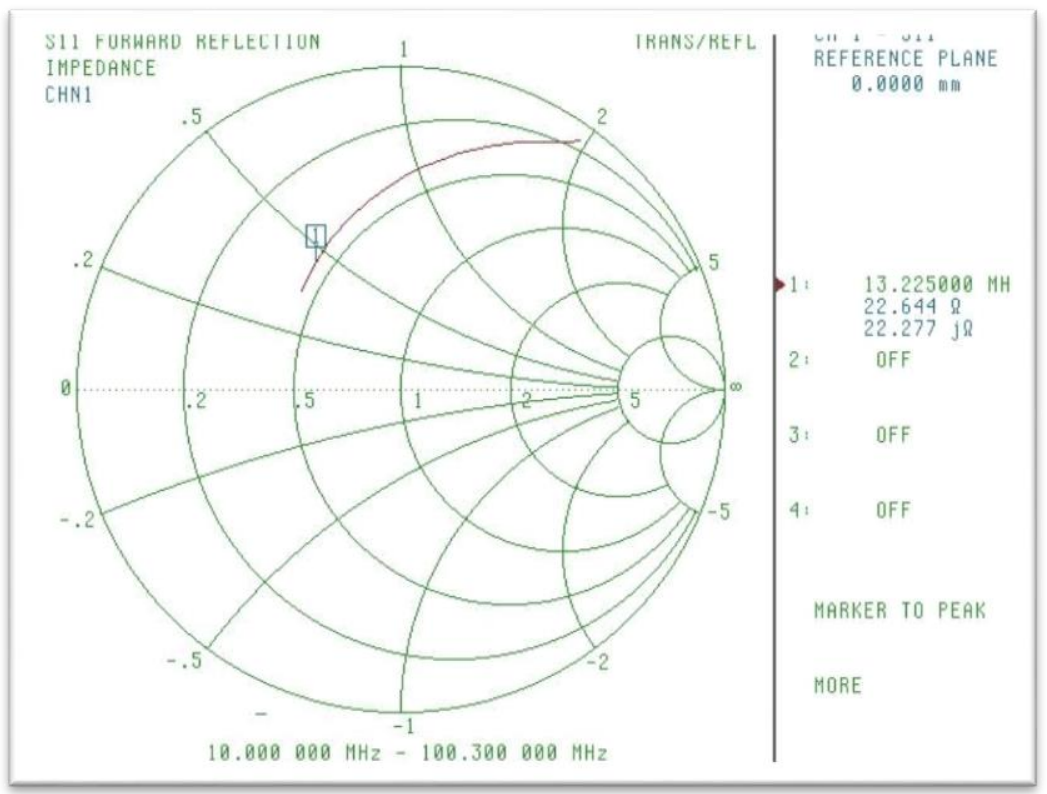

Figure 3.74 250 $\mu m$ Coil Design Inductance 
Unfortunately, the necessary $4 \mathrm{~V}$ peak signal was not obtained at the receive coil due to the high coil resistance from the thin traces. To resolve this matter, the $250 \mu \mathrm{m}$ coil design was used. Figure 3.75 shows test setup and a $4.7 \mathrm{~V}$ peak signal at the receive end when a $10 \mathrm{~V}$ peak, $20 \mathrm{MHz}$ signal was applied to the transmit coil.

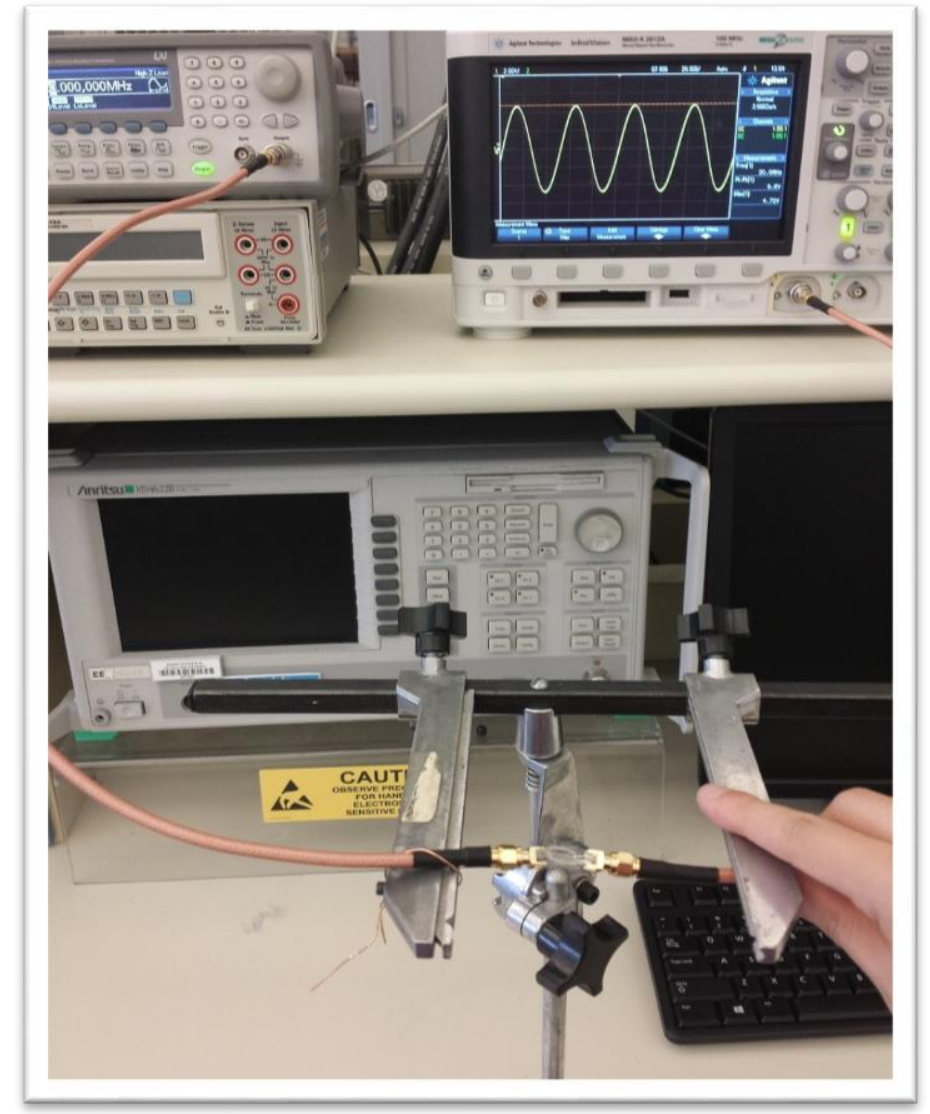

Figure 3.75 250 $\mu m$ Coil Design Inductance

\subsubsection{Polarization and Beam Width}

Polarization of the AUTs was tested by transmitting a $1 \mathrm{~dB}$ Sine wave at $50 \mathrm{~cm}$ with the reference antenna and measuring the received power as the AUT was rotated along the x-axis. Received power remained constant at every angle. From this, it was concluded that the AUT and the reference antenna were circularly polarized, which contradicts our theory that most dipole antennas are 
directional and our printed designs are basically dipoles. If either the reference antenna or AUT were directional, then we would not be able to receive any signals. Thus, beam width measurements are not applicable to circularly polarized antennas.

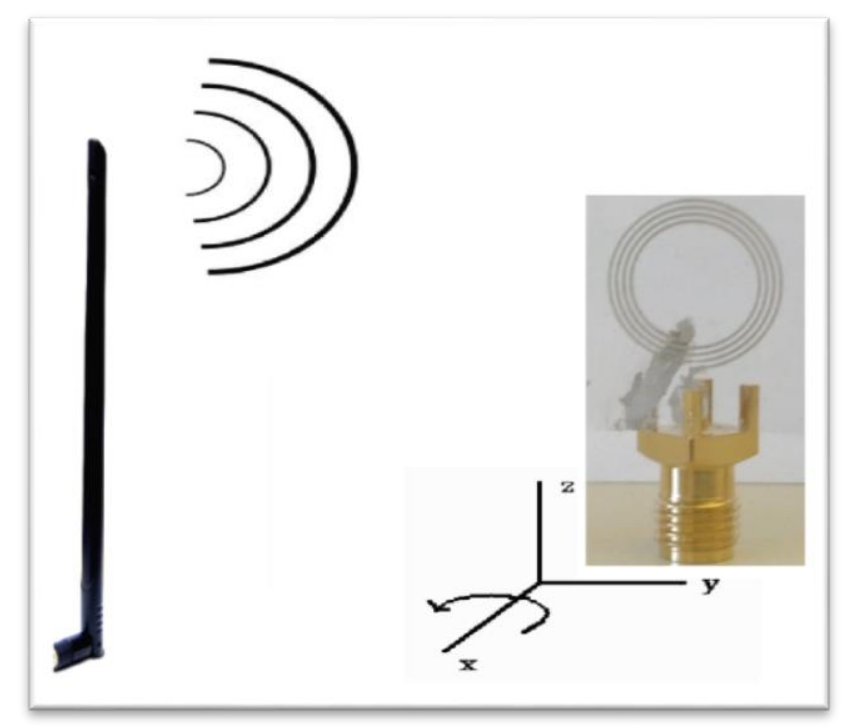

Figure 3.76 The AUT is rotated around the Horizontal Axis for Polarization Measurement

\subsubsection{Radiation Pattern (Magnitude)}

To characterize radiation patterns for the AUTs, a signal of $1 \mathrm{dBm}$ was transmitted from the AUT to the reference antenna at $65 \mathrm{~cm}$. The received signal was measured every 20 degrees of rotation around the reference antenna. The setup required both antennas to be connected to the frequency generator or VNA while orbiting the AUT. The setup was improved three times to find a consistent and efficient method of orbiting the antennas. 


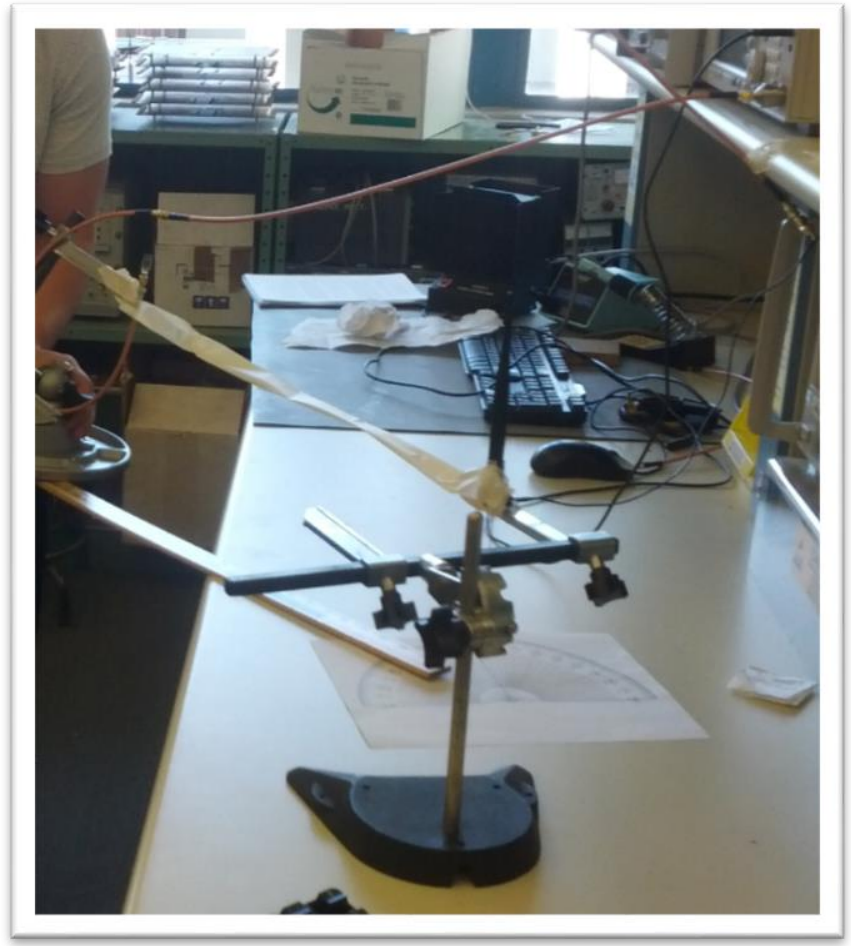

Figure 3.77 First Attempt Setup for Measuring Radiation Pattern

The first attempt at measuring radiation pattern required one person to manually hold the mount and ruler and another person to record the data. Unfortunately, the mount was heavy making it impractical to manually rotate the mount therefore rendering this setup unsuitable for acquiring data.

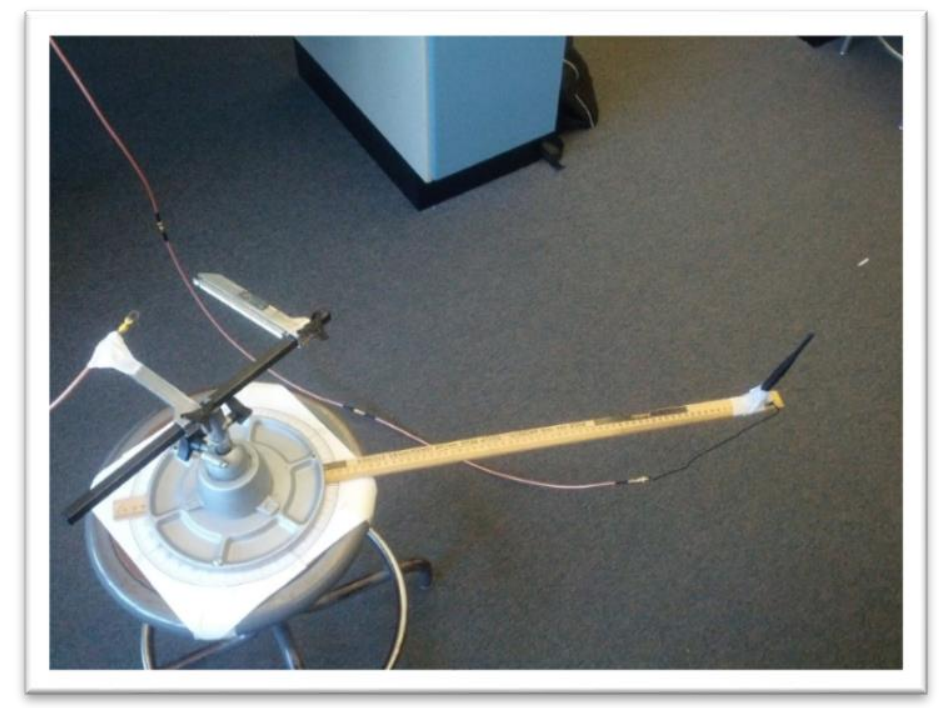

Figure 3.78 Resolved Setup for Measuring Radiation Pattern 
The final setup used a swivel stool to rotate the reference antenna, which was attached to a meter stick, around the AUT. The following radiation patterns were captured for AUT1, AUT2, and AUT3 in Figure 3.79, 3.80, and 3.81.

\begin{tabular}{r|r|}
\hline $\begin{array}{l}\text { Angle } \\
\text { [degree] }\end{array}$ & $\begin{array}{l}\text { Ref Rx Power from AUT 1 } \\
\text { [dBm] }\end{array}$ \\
\hline $\mathbf{0}$ & -53.88 \\
\hline $\mathbf{2 0}$ & -38.3 \\
\hline $\mathbf{4 0}$ & -41.67 \\
\hline $\mathbf{6 0}$ & -38.51 \\
\hline $\mathbf{8 0}$ & -41.55 \\
\hline $\mathbf{1 0 0}$ & -43.77 \\
\hline $\mathbf{1 2 0}$ & -53.4 \\
\hline $\mathbf{1 4 0}$ & -52.72 \\
\hline $\mathbf{1 6 0}$ & -48.93 \\
\hline
\end{tabular}

\begin{tabular}{|r|r|}
\hline $\mathbf{1 8 0}$ & -43.03 \\
\hline $\mathbf{2 0 0}$ & -46.76 \\
\hline $\mathbf{2 2 0}$ & -43.88 \\
\hline $\mathbf{2 4 0}$ & -42.86 \\
\hline $\mathbf{2 6 0}$ & -40.99 \\
\hline $\mathbf{2 8 0}$ & -42.14 \\
\hline $\mathbf{3 0 0}$ & -38.02 \\
\hline $\mathbf{3 2 0}$ & -39.98 \\
\hline $\mathbf{3 4 0}$ & -35.54 \\
\hline & -53.88 \\
\hline
\end{tabular}

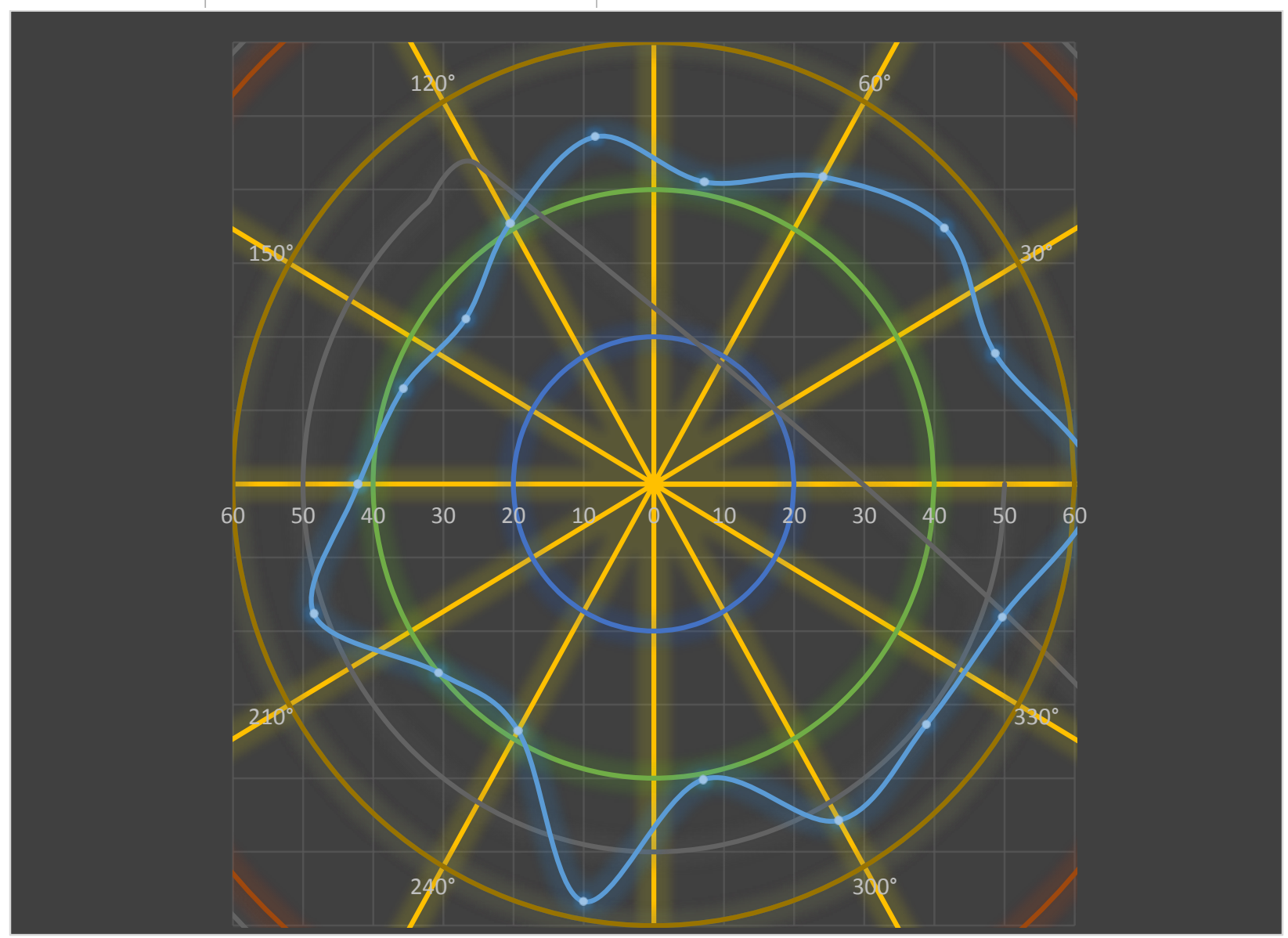

Figure 3.79 AUT1 Radiation Pattern and Measured Received Power 


\begin{tabular}{|r|r|}
\hline $\begin{array}{l}\text { Angle } \\
\text { [degree] }\end{array}$ & $\begin{array}{l}\text { Ref Rx Power from AUT 2 } \\
\text { [dBm] }\end{array}$ \\
\hline $\mathbf{0}$ & -55.52 \\
\hline $\mathbf{2 0}$ & -51.75 \\
\hline $\mathbf{4 0}$ & -68.91 \\
\hline $\mathbf{6 0}$ & -53.72 \\
\hline $\mathbf{8 0}$ & -50.1 \\
\hline $\mathbf{1 0 0}$ & -55.35 \\
\hline $\mathbf{1 2 0}$ & -54.6 \\
\hline $\mathbf{1 4 0}$ & -54.96 \\
\hline $\mathbf{1 6 0}$ & -52.41 \\
\hline
\end{tabular}

\begin{tabular}{|l|r|}
$\mathbf{1 8 0}$ & -63.36 \\
\hline $\mathbf{2 0 0}$ & -57.11 \\
\hline $\mathbf{2 2 0}$ & -62.48 \\
\hline $\mathbf{2 4 0}$ & -68.93 \\
\hline $\mathbf{2 6 0}$ & -59.54 \\
\hline $\mathbf{2 8 0}$ & -62.94 \\
\hline $\mathbf{3 0 0}$ & -59.55 \\
\hline $\mathbf{3 2 0}$ & -69.53 \\
\hline $\mathbf{3 4 0}$ & -62.74 \\
\hline $\mathbf{3 6 0}$ & -55.52 \\
\hline
\end{tabular}

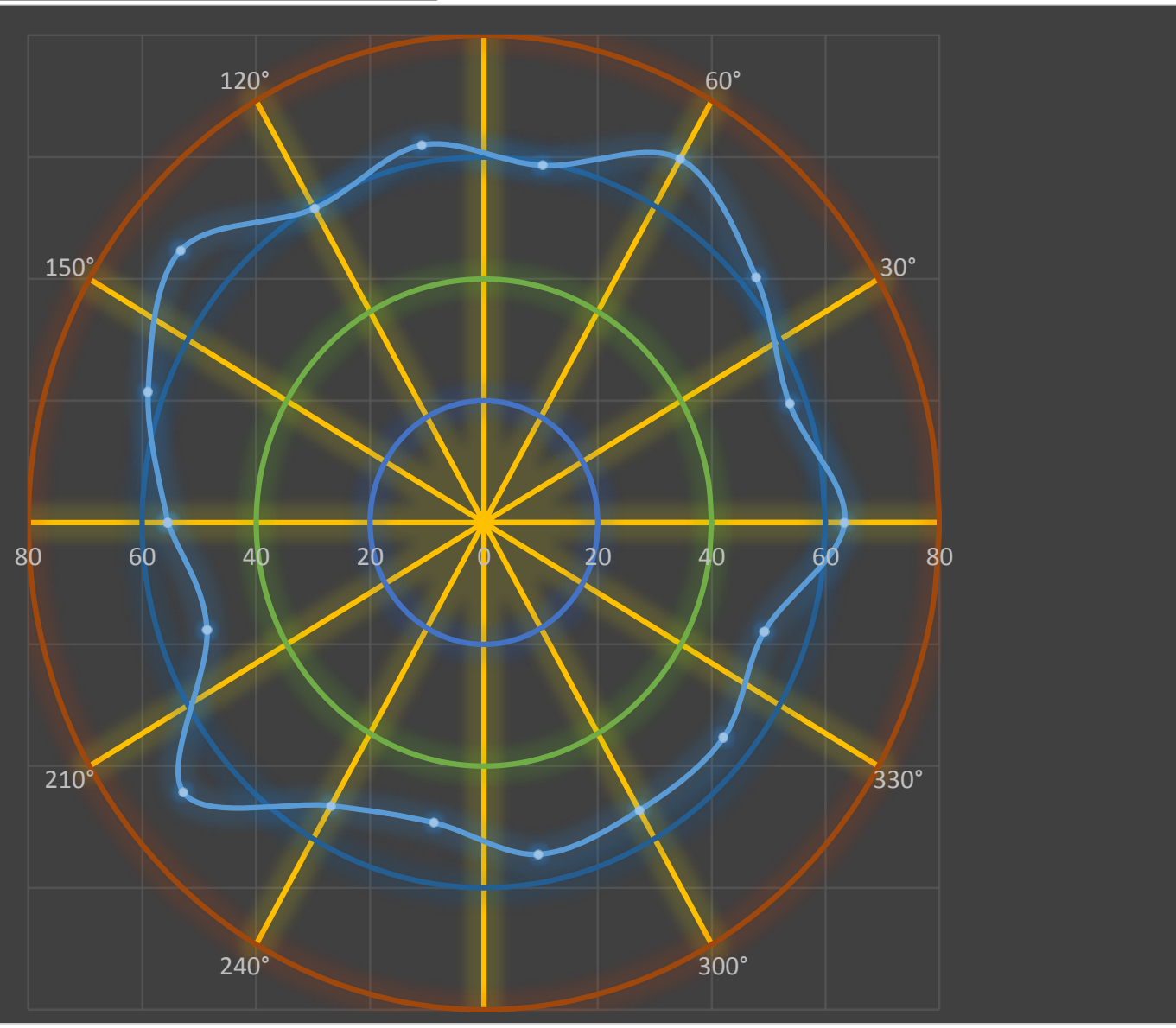

Figure 3.80 AUT2 Radiation Pattern and Measured Received Power 


\begin{tabular}{|r|l|r|r|}
\hline $\begin{array}{l}\text { Angle } \\
\text { [degree] }\end{array}$ & $\begin{array}{l}\text { Ref Rx Power from AUT 3 } \\
\text { [dBm] }\end{array}$ & $\mathbf{1 8 0}$ & -62.96 \\
\hline $\mathbf{0}$ & -42.12 \\
\hline $\mathbf{2 0}$ & -51.56 \\
\hline $\mathbf{4 0}$ & -39.97 & $\mathbf{2 0 0}$ & -51.76 \\
\hline $\mathbf{6 0}$ & -38.7 & $\mathbf{2 2 0}$ & -54.08 \\
\hline $\mathbf{8 0}$ & -57.62 \\
\hline $\mathbf{1 0 0}$ & -40.78 \\
\hline $\mathbf{1 2 0}$ & -52.79 \\
\hline $\mathbf{1 4 0}$ & -50.77 & $\mathbf{2 4 0}$ & -48.19 \\
\hline $\mathbf{1 6 0}$ & -52.88 & $\mathbf{2 6 0}$ & -41.67 \\
\hline & $\mathbf{2 8 0}$ & -47.91 \\
\hline & $\mathbf{3 0 0}$ & -40.94 \\
\hline
\end{tabular}

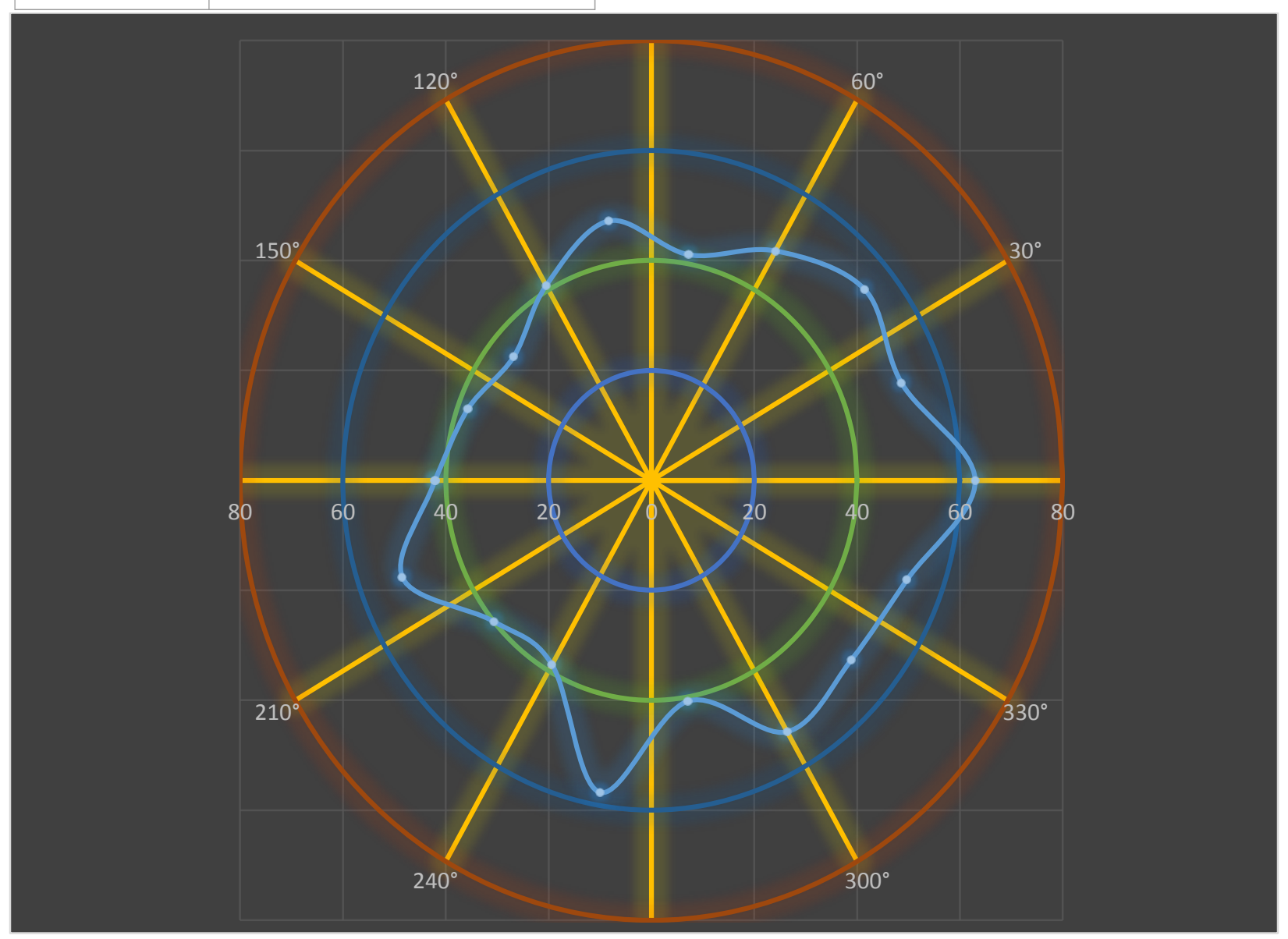

Figure 3.81 AUT3 Radiation Pattern and Measured Received Power 


\subsubsection{Efficiency}

Efficiency was calculated using measurements that were already collected. The gain of the transmitting reference antenna was $1.8 \mathrm{dBi}$, and the signal was $1 \mathrm{dBm}$. The required data for calculation is shown in Table 3 below.

\section{$\begin{array}{lll}\text { AUT1 } & \text { AUT2 } & \text { AUT3 }\end{array}$}

\begin{tabular}{|lccc|}
\hline $\begin{array}{l}\mathrm{S} 11(\Gamma) \\
{[\mathrm{dB}]}\end{array}$ & -5.722 & -2.346 & -3.476 \\
\hline $\begin{array}{l}\text { Received } \\
\text { power } \\
\text { [dBm] }\end{array}$ & -43.66 & -58.9 & -38 \\
\hline $\begin{array}{l}\text { Received } \\
\text { power [nW] }\end{array}$ & 279 & 1.3 & 158 \\
\hline $\begin{array}{l}\text { Reflected } \\
\text { Power [\%] }\end{array}$ & 7.2 & 33.9 & 20.2 \\
\hline gain [dBi] & 13.656 & 10.985 & 13.365 \\
\hline $\begin{array}{l}\text { pathloss } \\
\text { [dB] }\end{array}$ & -48.1 & -45.5 & -47.9 \\
\hline $\begin{array}{l}\text { pathloss } \\
\text { [nW] }\end{array}$ & -78.1 & -75.5 & -77.9 \\
\hline
\end{tabular}

Table 3 Measured Power Parameters for Efficiency Calculation

$P_{\text {out }}=P_{T X}+G_{\text {ref }}=1 \mathrm{dBm}+1.8 \mathrm{dBi}=2.8 \mathrm{dBm}$

$P_{65 \mathrm{~cm}}=P_{\text {out }}-P F=2.8 \mathrm{dBm}-48.1 \mathrm{dBm}=-45.3 \mathrm{dBm}=3 \mathrm{nW}$

$P_{\text {in }}=\frac{P_{\text {recieved }}}{1-\% \text { refected }}=\frac{279 e^{-9}}{1-7.2 \%}=\frac{279 e^{-9}}{92.8 \%}=300.6 \mathrm{nW}$

$\eta=\frac{P_{\text {out }}}{P_{\text {in }}}=\frac{3 \mathrm{nW}}{300 \mathrm{nW}}=1 \%$

\begin{tabular}{|l|l|}
\hline & Efficiency \\
\hline AUT1 & $1 \%$ \\
\hline AUT2 & Less than $1 \%$ \\
\hline AUT3 & $15.6 \%$ \\
\hline
\end{tabular}

Table 4 Summary of Tested Antenna Efficiency

The tested efficiency for AUT2 and AUT3 was close to the theoretical values from simulation. On the other hand, AUT1 failed to meet the expected efficiency because it was not properly tuned to $2.4 \mathrm{GHz}$ during 
the testing. The outer ring needed to be shorted by scrubbing off the silver.

The second iteration of printed antennas' gain were test by transmitting a $2.4 \mathrm{GHz} 10 \mathrm{dBm}$ signal using a $1.7 \mathrm{~dB}$ gain antenna at a distance of $42 \mathrm{~cm}$. In Figure 3.82 and 3.83 the detected signal powers for the printed antennas are shown.

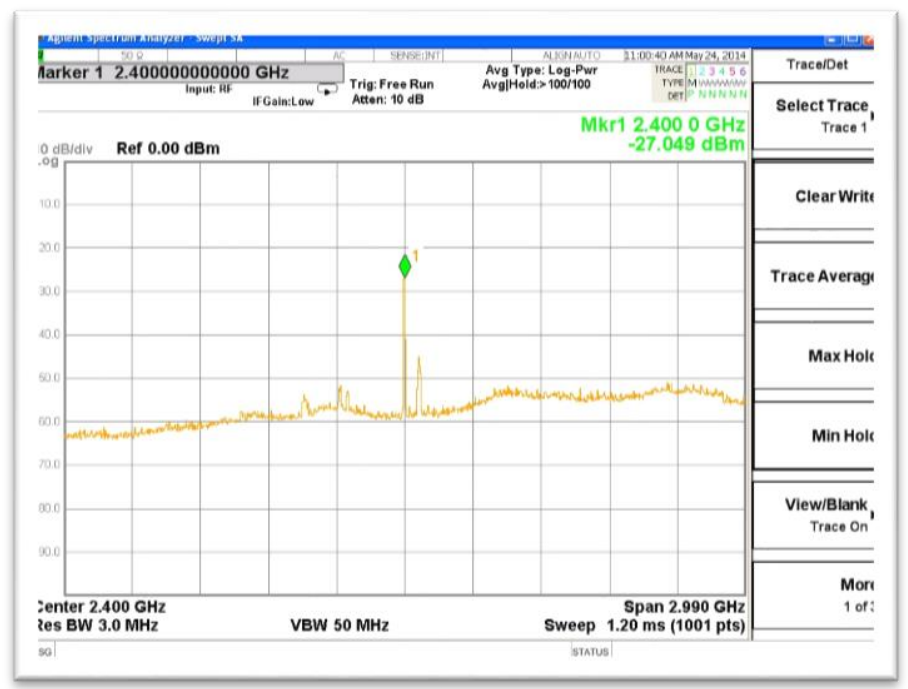

Figure 3.82 Received Signal Power at Spectrum Analyzer (IFA)

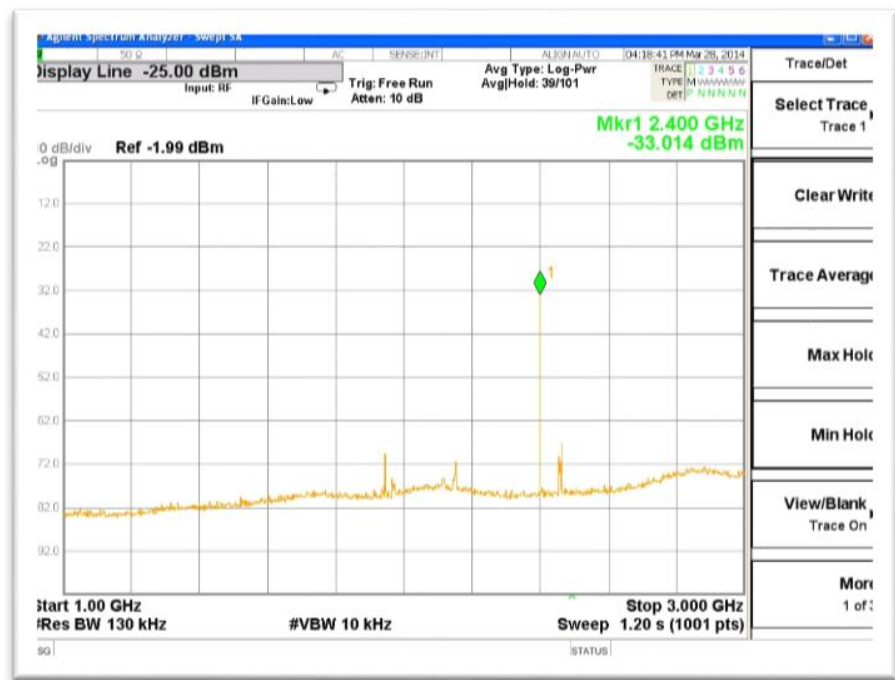

Figure 3.83 Received Signal Power at Spectrum Analyzer (Coil) 
The Friis transmission equation indicates that the power received is equal to the sum (in $\mathrm{dB}$ ) of antenna gains and signal power subtracted from the path loss [8].

$$
P_{R}(d B m)=P_{T}(d B m)+G_{T}(d B)+G_{R}(d B)-20 \log R(k m)-20 \log f(M H z)-32.44
$$

A distance of $42 \mathrm{~cm}$ attenuates the signal by $32.7 \mathrm{~dB}$ which means the signal right at the antenna has a power level of $-21 \mathrm{dBm}$ or $7.95 \mu \mathrm{W}$. $\left|\mathrm{S}_{11}\right|$ of $-9.5 \mathrm{~dB}$ means that $67 \%$ of the power is accepted by the antenna resulting in $6.7 \mu \mathrm{W}$ or $21.7 \mathrm{dBm}$ of accepted power. Therefore, the efficiency of the IFA can be calculated to be $33.5 \%$ resulting in a total antenna gain of $-5.3 \mathrm{~dB}$ which is $3.3 \mathrm{~dB}$ higher than simulation. Similarly, the total gain of the printed coil design was calculated to be $-13 \mathrm{~dB}$. 


\section{Chapter 4}

\subsection{Power Supply}

During the research phase, different types of portable power supplies (batteries and capacitors) were compared and contrasted. Not only was power, size, and flexibility a concern, but also health hazards from the materials being used. Graphene super capacitors represent a potential answer to these requirements. Super capacitors have unique properties that make them an attractive choice as a power source. They are essentially maintenance-free, possess a longer cycle-life, require a very simple charging circuit, experience no memory effect, and are generally much safer [10]. Also, one of the key characteristics of a super capacitor is that it can be charged and discharged at high rates.

During the attempts pristine graphene and thin films were successfully created as well as chemically bonding graphene with other compounds. During the first iteration only $273 \mathrm{uF}$ was achieved with a micro-fabricated super capacitor with pristine graphene and ionic liquid that were encapsulated in PDMS. Attempts with printing were also utilized and it was found for prototyping that printing thin film batteries or super capacitors have less challenges than microfabrication due to the surface energy of the material. It was found that even though Super capacitors beat a secondary battery in cycle lifetime, flexibility, and charge rate, the battery has better operation time due to its slow discharger rate. Therefore, implementing a battery from Cymbet as a steady power 
source and have the super capacitor when you need extra power for driving the display or transmitting from the lens.

Unfortunately, graphene super capacitors are highly experimental. Even after conducting large amounts of research into integrating the technology into our lens there was concern of great probability that the component might not be fabricated in a reasonable time frame. Therefore a backup power supply was decided using a thin film lithium ion batteries, which are readily mass produced by manufacturer Cymbet. While not as flexible and thin as a graphene super capacitor, thin film lithium ion batteries are affordable and easy to use, making them a good choice to demonstrate our embedded power platform. One such battery is shown in Figure 4.1 below.

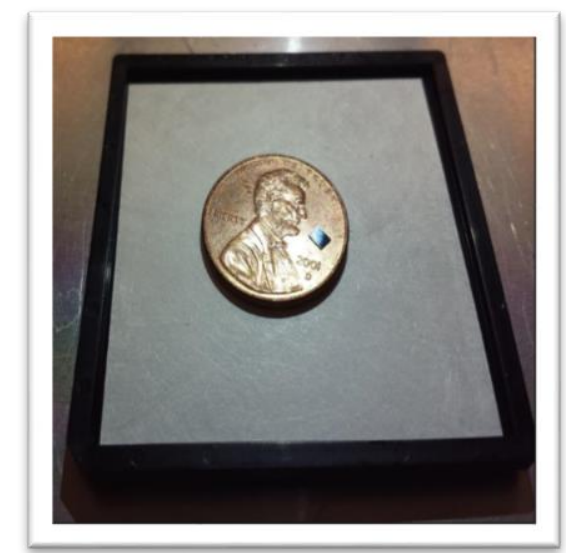

Figure 4.1 Thin Film Lithium Ion Battery with Penny for Scale

\subsection{Background}

Graphene is a two dimensional crystalline allotrope of carbon where the atoms are densely packed in a sp2-bonded hexagonal pattern [11]. Graphene can be described as the top skin layer of graphite. High quality 
graphene (pristine graphene) is not only an excellent conductor, but it is also strong, flexible and nearly transparent. The conductivity of a singlelayer graphene sheet has been measured up to $649 \mathrm{~S} / \mathrm{cm}$ [12]. The most important property of graphene with respect to super capacitors is the high theoretical specific surface area of $2675 \mathrm{~m}^{2} / \mathrm{g}$ which corresponds to a theoretical specific capacitance of $550 \mathrm{~F} / \mathrm{g}$.[10] Note that target capacitance value for the fabricated super capacitor calculated was $32 \mathrm{mF}$.

Secondary batteries are batteries that are able to recharge by reversing the chemical reaction of the cathode and anode. Batteries are consistent power sources that give a steady amount of voltage for the duration of time operated. This is due to is slow charge/discharge rate. Another advantage that batteries have, especially lithium ion batteries, are their large energy density for charge storage relative to other types of batteries. The disadvantages are its life cycles meaning every time you charge the battery and deplete it, the energy density we decrease permanently every certain amount of cycles.

For characterizing the electrochemical capacitors, the performance will be evaluated by cyclic voltammetry, galvanostatic charge/discharge curves using an electrochemical workstation. The sheet resistance and conductivity values can be calculated by measuring the resistance with a probe and the film thicknesses with an interferometer (can measure film thicknesses greater than 1000 angstroms). The graphene structured 
electrodes will be mapped out and imaged with a scanning tunneling microscope (STM). Final demo test will interface an equivalent circuit (to model all parts of the system), consisting of single components that will match both real and imaginary impedance and one led will the same power ratings. It will be timed and measured to see how long the power source will run the LED.

The capacitance of the device is calculated from the galvanostatic curves at different current densities using the following formula:

$$
C=i /\left(-\frac{d V}{d t}\right)
$$

Where $i$ is current applied (A), $d V / d t$ is the slope of the discharge curve $(\mathrm{V} / \mathrm{s})$.

Specific capacitance is based on the area and volume of the device stack according to the following formulas:

$$
\begin{gathered}
\text { Area Capacitance }=C / A \\
\text { Volumetric Stack Capacitance }=C / V
\end{gathered}
$$

Where $A$ is the area of the device $\left(\mathrm{cm}^{2}\right)$ and $V$ is the volume of the device $\left(\mathrm{cm}^{3}\right)$.

The power of the device was calculated from the galvanostatic curves at different charge/discharge current densities using the following formula:

$$
P=(\Delta E)^{2} / 4 R_{E S R} V
$$

Where $P$ is the power $\left(\mathrm{W} / \mathrm{cm}^{3}\right), \Delta E$ is the operating voltage window (measured in volts and obtained from the discharge curve excluding the 
I $R$ drop), $V$ is the volume of the stack $\left(\mathrm{cm}^{3}\right)$, and $R_{E S R}$ is the internal resistance.

The energy density of the device can be obtained from the following formula:

$$
E=C_{v}(\Delta E)^{2} /(7200)
$$

Where $E$ is the energy density $\left(\mathrm{Wh} / \mathrm{cm}^{3}\right), \mathrm{CV}$ is the volumetric stack capacitance $\left(F / \mathrm{cm}^{3}\right)$, and $\Delta E$ is the operating voltage window $(V)$.

\subsection{Manufacturing Process of Graphene}

\subsubsection{Synthesis using Chemical Vapor Deposition}

For the electrodes, there are three essential steps for the growth and transfer of grapheme. The first step is chemical vapor deposition, the second is the adhesion of polymer thermal tape on the grapheme and etch the copper foil layers, and third is releasing the grapheme layer and transferring it to the desired substrate.

In the CVD process a machine known as the Aixtron was used to synthesize a monolayer grapheme film on a copper substrate that was the size of a 4 inch wafer. The copper foil wafer is inserted into the quartz tube and heated to $1,000^{\circ} \mathrm{C}$ flowing 8 s.c.c.m. $\mathrm{H}_{2}$ at 90 mtorr. When the temperature reaches $1,000^{\circ} \mathrm{C}$, the sample is annealed for $30 \mathrm{~min}$ without changing the flow rate or pressure. By heat treating the copper foil, the grain size increases from a few micros to approximately $100 \mu \mathrm{m}$.

Previous researched experiments have found that the larger the grain 
size in the copper substrate, the higher the quality of the grapheme field is yielded. The gas mixture of $\mathrm{CH}_{4}$ and $\mathrm{H}_{2}$ was flown at 460 mtorr with rates of 24 and 8 s.c.c.m. for $30 \mathrm{~min}$. After, the sample was rapidly cooled to room temperature by flowing $\mathrm{H}_{2}$ under a pressure of 90 mtorr.

Once synthesis had occurred in the copper substrate, it was attached to a thermal release tape apply a pressure of approximately $0.2 \mathrm{MPa}$. The copper was removed with an aqueous $0.1 \mathrm{M}$ ammonium persulphate solution $\left(\mathrm{NH}_{4}\right)_{2} \mathrm{~S}_{2} \mathrm{O}$. Finally, the graphene film is transferred from the polymer thermal tape on the desired target substrate by using of heat (thermal treatment at $90-120^{\circ} \mathrm{C}$ ) to remove the adhesive force holding the film. Unfortunately, characterization of graphene was still being researched as well as its applications. At the time of synthesis newly published research showed that pristine graphene was not suitable as an electrode compared to graphene films with defects. The reason is that pristine graphene surface area that the ions interact with is less than the film with defects. This method was abandoned and a chemical synthesis was used to create the electrode film.

\subsubsection{Chemical Synthesis of Graphene Film}

The second method involves chemically exfoliating a lump of graphite into thin sheets of graphene that can be used to create a thin film. Figure 4.2 below illustrates the process. 


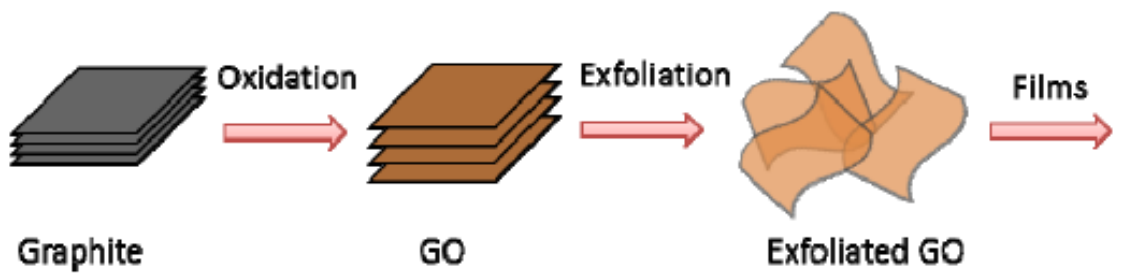

Figure 4.2 Steps for Synthesis of Graphene Film

Preparation of Graphene Oxide. Graphene oxide was prepared from natural graphite flakes using a modified Hummers method. In a typical synthesis procedure, $1.0 \mathrm{~g}$ of graphite flakes was treated with a mixture of concentrated sulfuric acid $(30 \mathrm{~mL})$ and nitric acid $(10 \mathrm{~mL})$ at room temperature under ultra-sonication for 3 hours. The acid-treated graphite flakes were rinsed with deionized (DI) water five times to collect the paste-like solid. The dried solid sample was then added into $200 \mathrm{~mL}$ of concentrated sulfuric acid at $0{ }^{\circ} \mathrm{C}$, in which $10 \mathrm{~g}$ of potassium permanganate was subsequently added under $20^{\circ} \mathrm{C}$. The mixture was then heated to $35^{\circ} \mathrm{C}$ and stirred at this temperature until the mixture became light brown in color with a significant increase in viscosity. To terminate the oxidization reaction, $200 \mathrm{~mL}$ of DI water was added to the mixture under stirring for $30 \mathrm{~min}$ in an ice bath, followed by drop-wise addition of $3 \mathrm{~mL}$ of $30 \%$ hydrogen peroxide. To collect the product, the dispersion was centrifuged to produce a bright yellow precipitate, which was then washed with $3 \mathrm{~mL}$ of $1.0 \mathrm{M}$ hydrochloric acid. The precipitate was further washed with DI water until the $\mathrm{pH}$ reached $\sim 5$. After 
centrifugation, the gel-like aqueous dispersion of graphene oxide was obtained [13].

The film was developed on solid uniform surface that was dried for 3 hours at $180^{\circ} \mathrm{C}$. The resulting graphene film is shown in Figure 4.3 below.

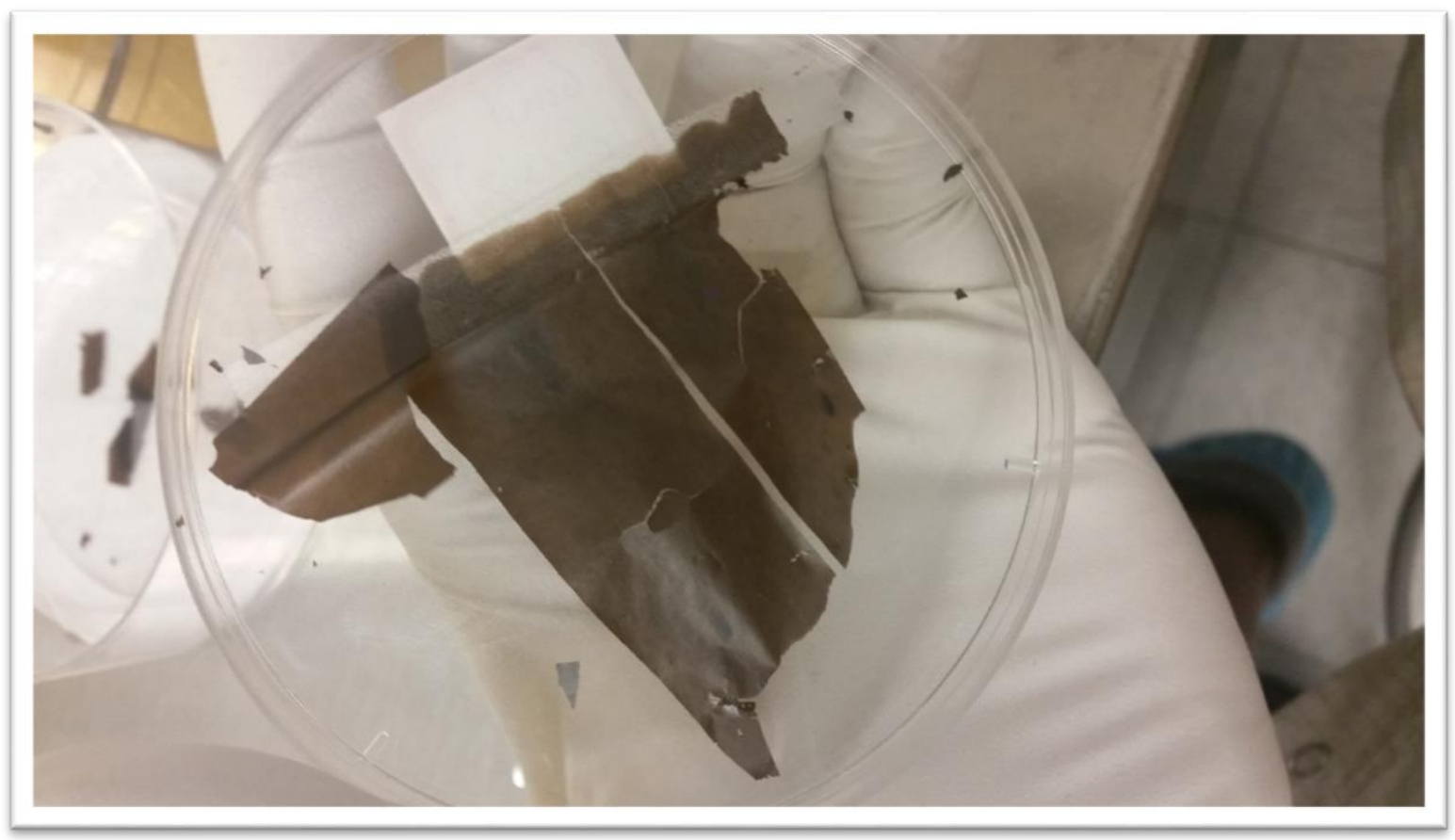

Figure 4.3 Synthesized Graphene Film

\subsection{Synthesis of Graphene Base Electrode}

This section is not present due to pursuit of a utility patent.

\subsection{Result of Graphene Based Electrode Super Capacitor}

This section is not present due to pursuit of a utility patent. 


\section{Chapter 5}

\subsection{Electro-Chromic Ink Display}

The display used for the final iteration of the lens was the electrochromic (EC) ink display which had several advantages compared to the alternatives. This type of display uses electro-chemical reactions from the interaction of the electrodes with the ions in the electrolyte. This resulted in a visual macro-scale change in the display. The display utilized screen printing technology which required less processing steps and materials relative to microfabrication processing. Another key advantage of the EC ink display is its transparency and is visually colorless when no charge is stored in the cell. When 1-3 volts are applied the colorless transparent display turns into a transparent blue color. It should be noted that a potential above 3.5 volts could possibly damage the screen burning the PEDOT and silver traces interface connections to the PEDOT. For the initial prototypes the EC ink display was composed of three layers that are similar to a battery topology, the current collector, electrode and electrolyte. The final iteration for the lens simply consisted of the electrode and the electrolyte layers as shown in fig. 5.1a and 5.1b. 


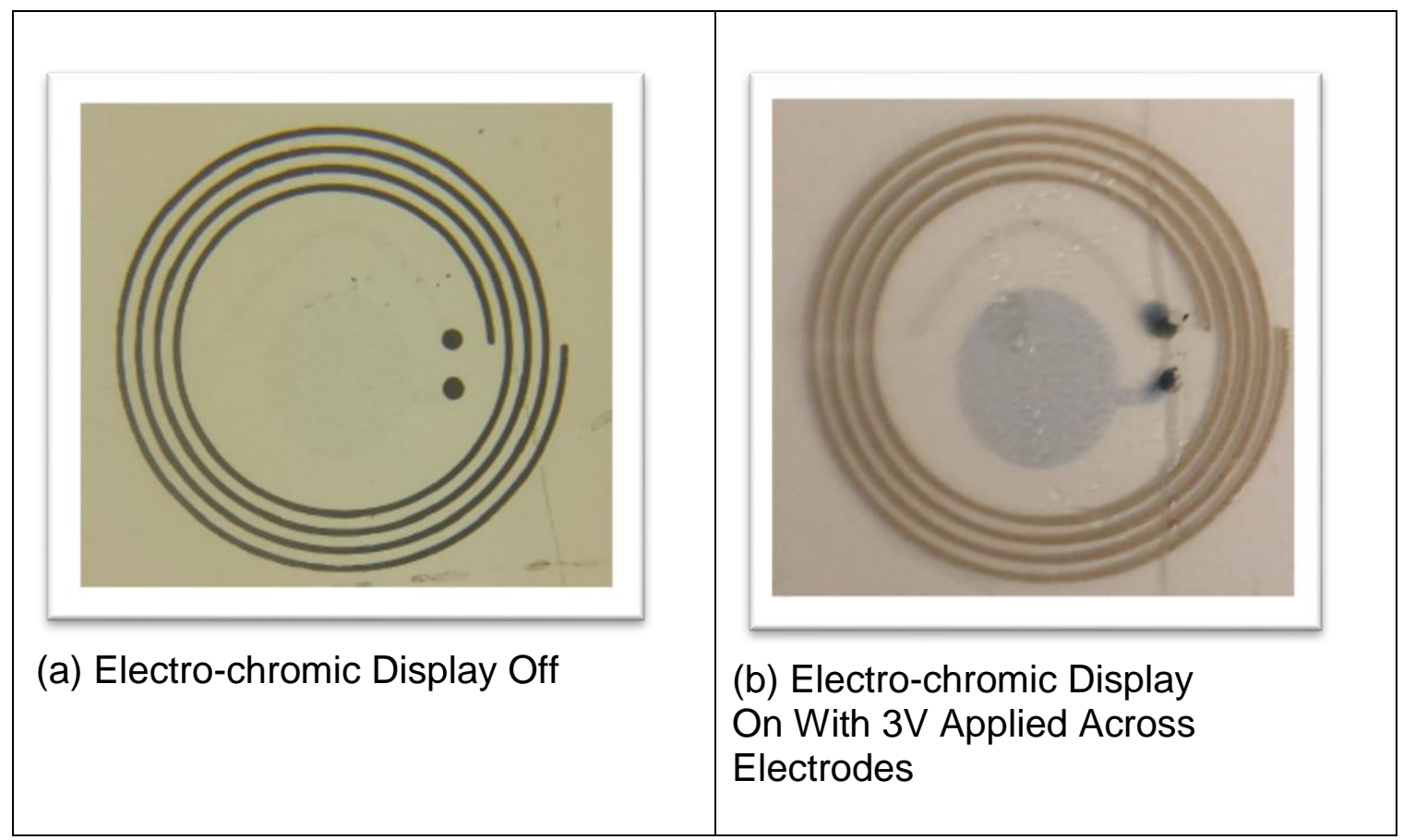

Figure 5.1 Stages of Operation for Electrochromic Display

Figure 5.1a and Figure 5.1b also shows the charged and discharged state of the EC ink display. The state of the display holding a charge was able to last for greater than 30 minutes from the intial charge time. The life time of the display with encapsulation was over 9 days and still able to function. With proper encapsulation the lifetime of the display would be longer. Another attempt of the increase the lifetime of the display is to add a gel seperator. This prevented the pathway medium from drying allowing the ions to flow to either electrodes based on potential polarities. Since the EC ink display is a two layer configuration, two step printing is the required minimum for manufacturing. The conductive polymer used was the Clevios $^{\mathrm{TM}}$ PEDOT:PSS by Heraeus which was diluted with methanol 50 wt $\%$. After printing the PEDOT:PSS, the electrodes were cured in the oven at 80 degrees Celsius for approximately 10 minutes. The final step was printing the 
electrolyte layer. The optimized formula mixture for the electrolyte consisted of a $15 \mathrm{wt} \%$ of polymethyl methacrylate (PMMA), $84.5 \mathrm{wt} \%$ of propylene carbonate, and 0.5 wt\% of Lithium Perchlorate $\left(\mathrm{LiClO}_{4}\right)$.

\subsection{Functionality of Device}

Initially there were two different concepts for the lens display. The first was using a single surface mount LED component. If the threshold trigger was activated then the LED would turn on and off at a 50 percent duty cycle at around $60 \mathrm{~Hz}$ frequency. However, even with 50 percent duty cycle, the LED required a continuous amount of current for operation. The current would consume the battery's power in a short amount of time.

The second concept was using an experimental display made of different polymer inks and an electrolyte. This display had many benefits over the surface mount LED. One being that the electro-chromic ink display, in its zero charge state, is visually clear and transparent. This enables more usable real-estate on the lens and could be placed within the field of vision area. Also, the display does not require a constant current source, unlike the surface mount LED. As well as fabricating the display on a transparent substrate, which can operate while being flexed. 


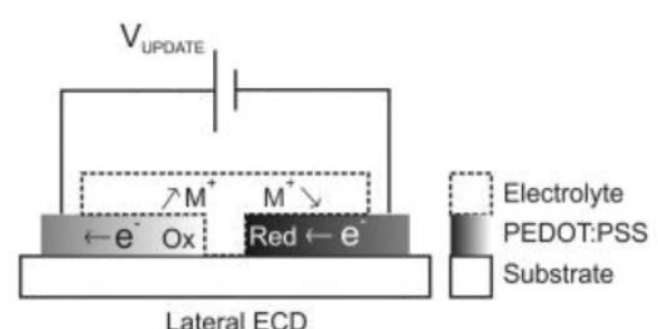

Lateral ECD

Figure 5.2 Electro-chromic Display Layers

Before going over the manufacturing of this device, I would like to explain the background of how this display works. The above figure 3.2 shows a cross-sectional side view of the display with a power source and layer description. As stated earlier, the electrodes are made from PEDOT which is an organic polymer with conductive properties. Poly(3,4ethylenedioxythiophene) chemically doped with poly(styrene sulfonic acid) (PEDOT:PSS) is a material system commonly used as a conductive and transparent coating in several important electronic applications. The material is also electrochemically active and exhibits electrochromic (EC) properties making it suitable as the active element in EC display applications.[14] This conductive polymer acts as both an electrical conductor as well as the electro-chromic material. The electrolyte is printed over the PEDOT:PSS electrodes to bridge the gap between the electrode with a medium that has a high ionic conductivity. When a potential is applied to the electrodes an electrochemical reaction takes place known as a redox reaction. A redox reaction, also known as an oxidation-reduction reaction, is a set of chemical reactions that are concerned with the transfer of electrons between species causing an 
atom's oxidation state to change. Oxidation refers to the loss of electrons while reduction refers to the gain of electrons. Each reaction occurs simultaneously in the redox and are individually referred to as a halfreaction because the two half-reactions are needed to form the whole reaction. In Figure 5.3 below, shows a cross-section of a display with the initial state of the display and the state after the electrochemical reaction takes place.

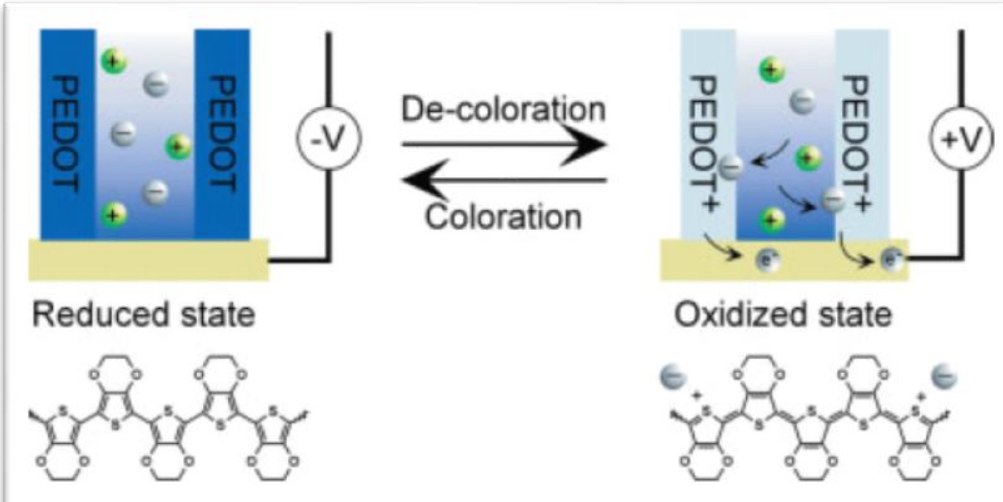

Figure 5.3 Redox Reaction in EC Display

As a positive potential is applied to the display, shown on the right figure, the redox reaction takes place transitioning the PEDOT from clear to blue. The reaction could be reversed, shown in the left figure, when a negative potential (or ground) is being applied.

\subsection{Fabrication Using Printed Technology}

The following sections explain the manufacturing procedures as well as optimization techniques of the display. This section is divided into the following three subsections: PEDOT electrode optimization, electrolyte development and optimization, and printing technology process. 


\subsubsection{PEDOT Electrode Optimization}

The purpose of this subsection demonstrates different characterization tests on the given PEDOT:PSS material in order to obtain the highest contrast ratio when a electrochemistry reaction took place. One of the initial problems from the first EC ink display prototype was the layer of PEDOT:PSS not being homogenous after screen-printing. On macro scale, there were visual implications in different regions of the display which became blue instead of clear colorless even though the display was fully discharged.

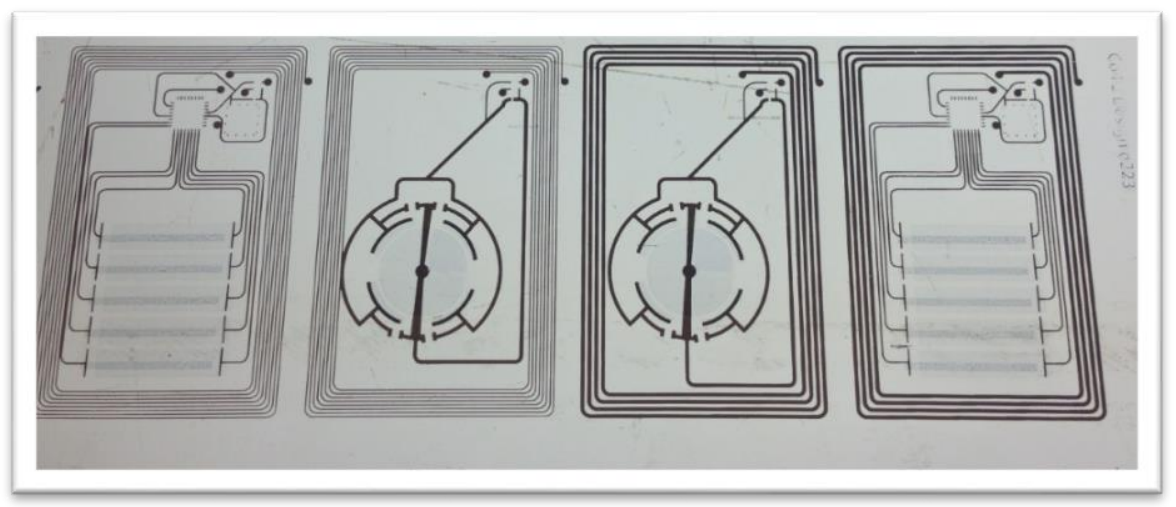

Figure 5.4 Defects in PEDOT:PSS

Research had found that as the PEDOT layer became thinner in the range of 500 to $2000 \mathrm{~nm}$ [14], the best contrast ratio could be obtained. Figure 5.5 shows the relationship between the PEDOT layer pixel thickness relations to the optical contrast of the display. 


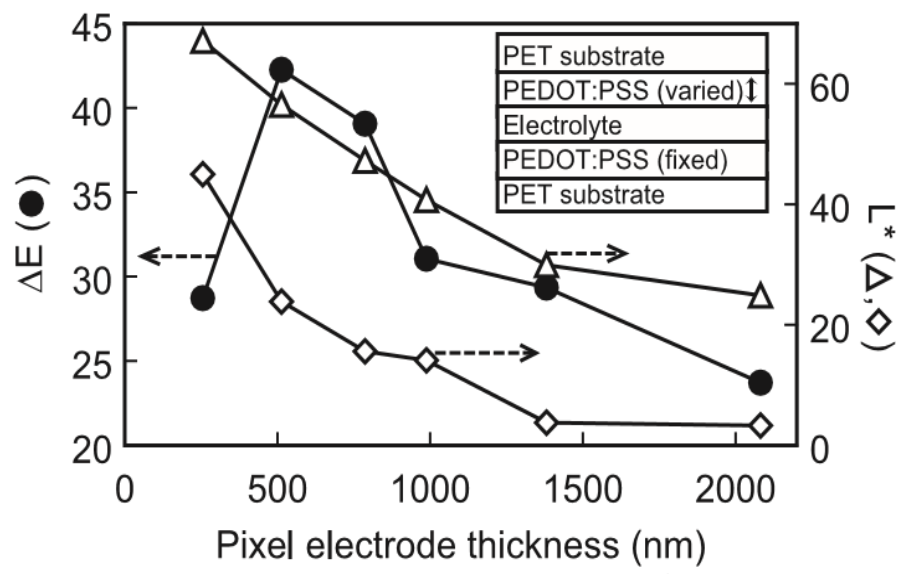

Figure 5.5 Color contrast $(\triangle E)$ and lightness $\left(L^{*}\right)$ vs. the PEDOT:PSS thickness of the pixel electrode

The Black filled circles represent $\Delta \mathrm{E}$, while triangles and diamonds denote the $L^{*}$ values of the white and colored state, respectively. A bias of +2.2 and $1.2 \mathrm{~V}$ was used to obtain the electrochromic color switch between the fully reduced and the fully oxidized states. The inset shows the cross section image of the EC display used in this measurement [14]. The process for spin coating the PEDOT:PSS on a PET substrate was determined by using the following equation to find the angular velocity and time.

$$
d=\frac{1}{\sqrt{\frac{4 \rho \omega^{2}}{3 \mu}} t}
$$

Where $d$ is the thickness, $\rho$ is the specific gravity, $\mu$ is the viscosity, $\omega$ is the angular velocity, and $t$ is the time measured in seconds. From the equation, I was able to create an excel spread sheet to determine what viscosity we needed the PEDOT:PSS to be in order to spin the desired pixel layer thickness of .6 microns in the spin coater. Note that the excel spread sheet can be referred to in Appendix A. 


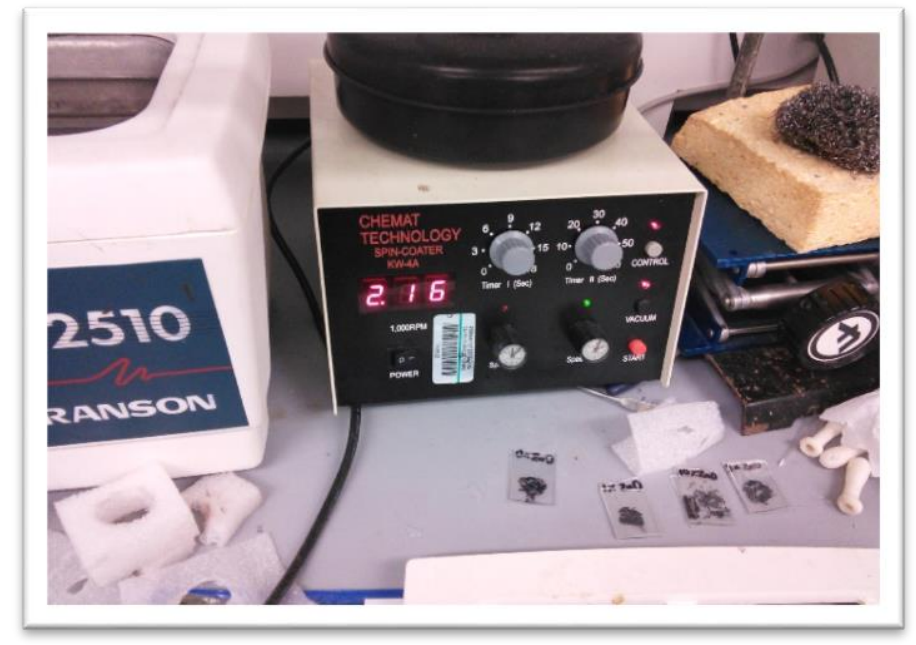

Figure 5.6 Spin Coater used to Create PEDOT Film

The best resulting film was using a 1:1 ratio of PEDOT:PSS and methanol mixture that was placed on a square piece of PET. The mixture was applied on by use of a syringe and initially spun at 500RPM for 60 seconds and planarized at 3000RPM for 60 seconds.

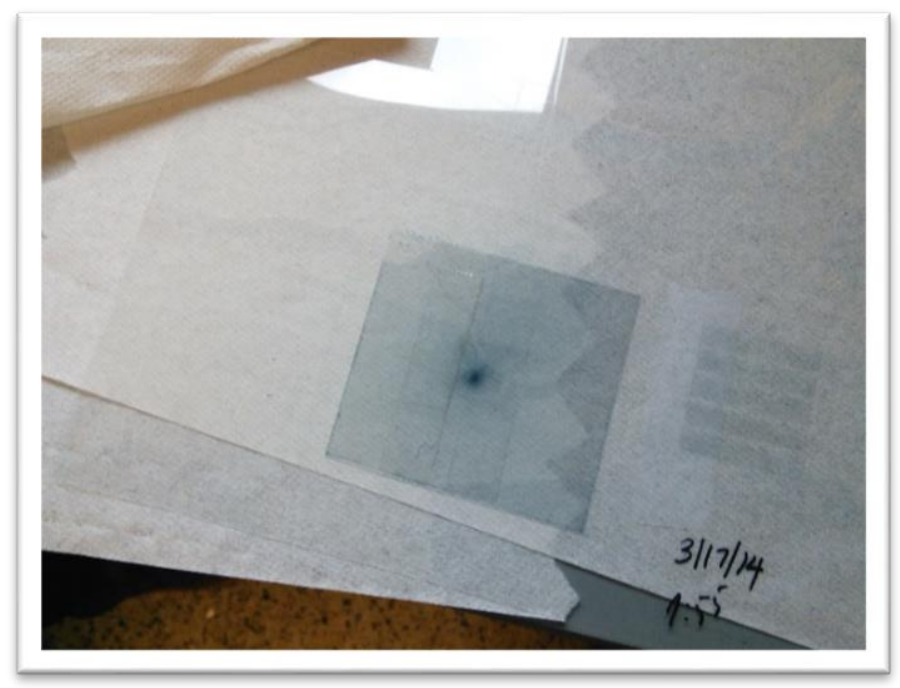

Figure 5.7 Results of a 1:1 ration of PEDOT and Methanol

The resistance was measured and the results were the thinner film had higher resistance than the current mixture of PEDOT:PSS being used. Since a spin-coater was not an attainable instrument in the Graphic 
Communication Department, one of the best approaches was to dilute the concentration of PEDOT:PSS material and decrease the viscosity. A functioning display had already been obtained in which Ethanol (a nontoxic chemical solution) was used to make the dilution, this yielded a high rate of success to this approach.

At the time, if the performance of this diluted PEDOT:PSS material was not satisfactory, other polymer resins such as polystyrene or polyurethane would have been used to dilute the PEDOT content while maintaining the viscosity of the material.

After printing the diluted PEDOT:PSS, three different tests will be performed on PEDOT:PSS layer. The first test was a visual inspection which checked if the PEDOT film was homogeneous. This was verified using an optical microscope and can be seen in the Figure 5.8 below.

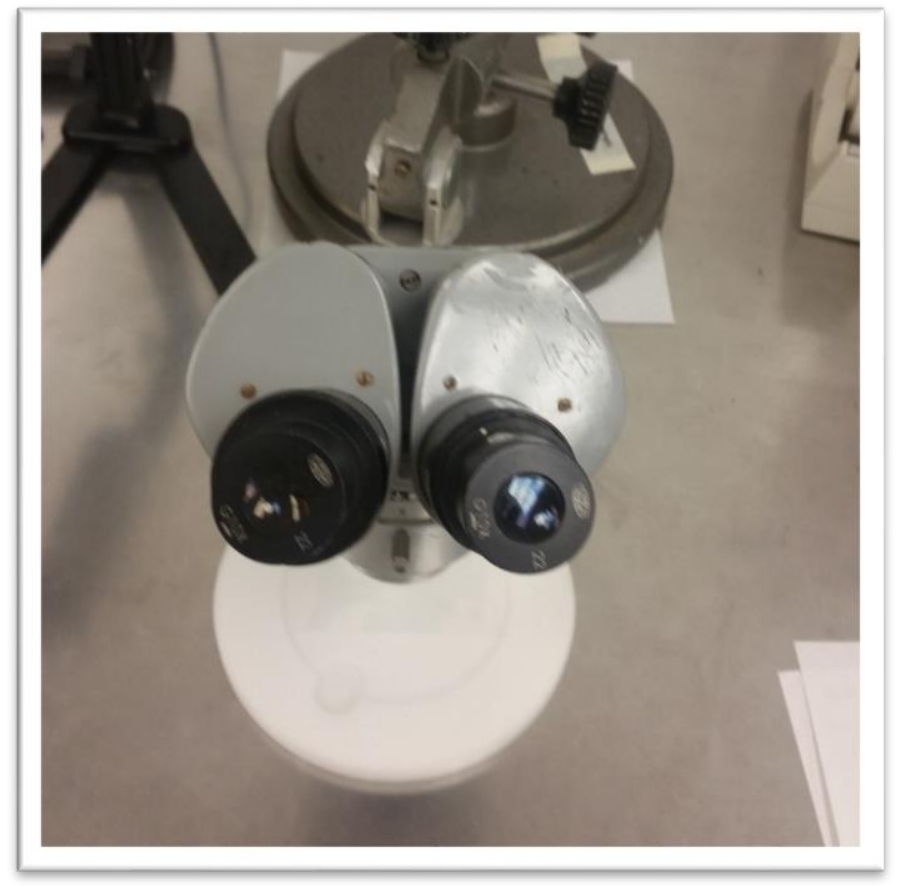

Figure 5.8 Optical Microscope Verification of Uniformity in Film 
It was then followed by a second test, resistance determination using the equipment shown in Figure 5.9. The final test was to determine the charge and discharge time.

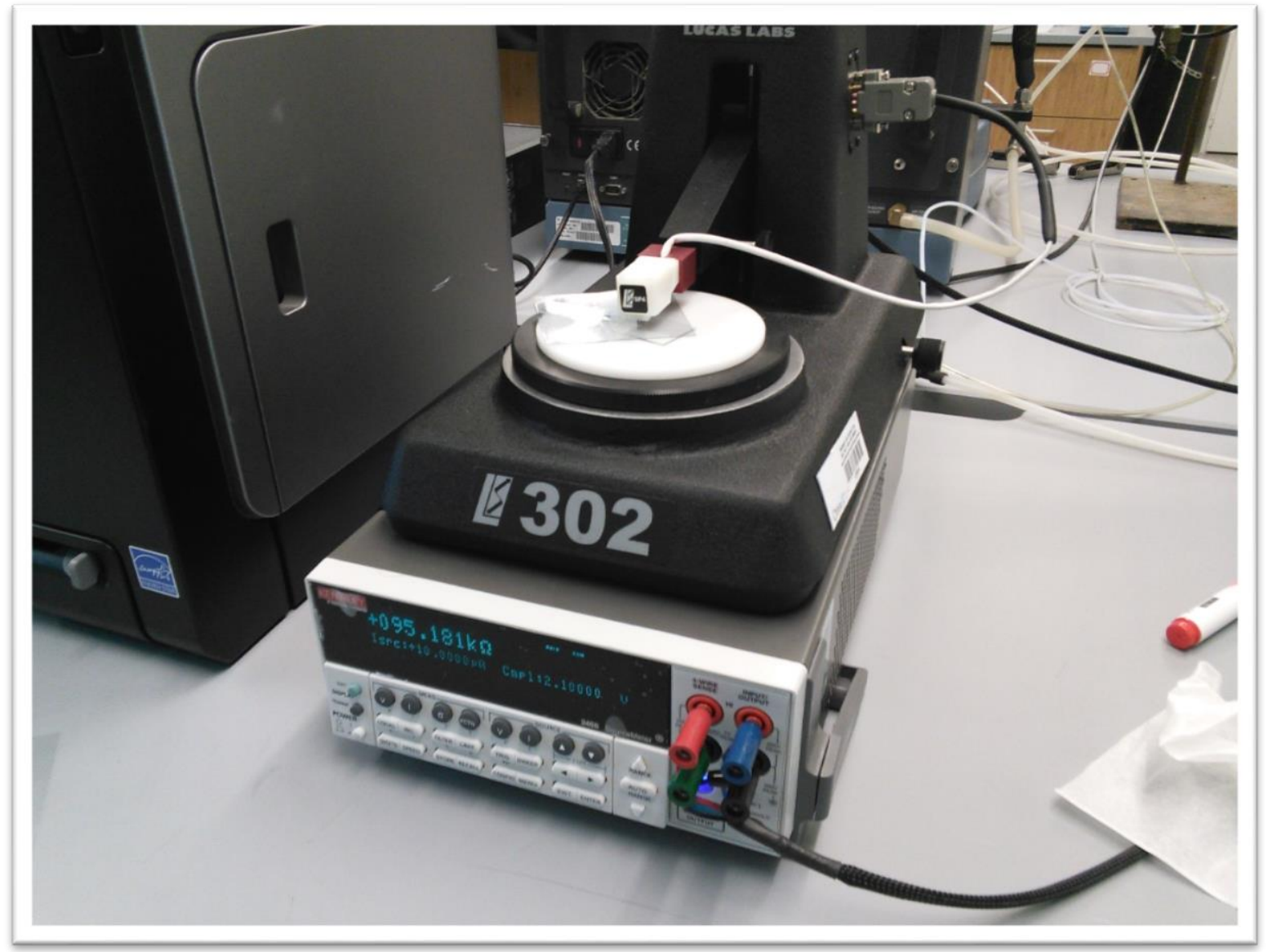

Figure 5.9 4-Point Probe Used to Measure Resistance

\subsubsection{PEDOT:PSS Testing Results}

In order to improve PEDOT:PSS layer properties, the viscous solution was diluted with $50 \mathrm{wt} \%$ methanol. Once PEDOT:PSS layer was printed, a visual inspection was performed. The printed film was homogenous and no defects observed. The resistance of the film was measured by a 4 point probe multimeter and showed an average resistance value of 95 kiloohms. The charge time was dependent on the thickness of 
PEDOT:PSS layer, the applied electrolyte and the circuit design. For initial design design, the transition time from blue to clear EC display was about 1:30 minutes. Once thinning the layer to $600 \mathrm{~nm}$, the transition time was less than 1 seconds making its response more than two orders of magnitude faster. This coincided with the data acquired from research done by Seung Cho [15].

PEDOT and its derivatives are an ideal electrochromic material of conducting polymers for electronic paper due to good color, mechanical stabilities and facile fabrication. Research from [14] determined that decreasing the thickness of the electrode would lead to a faster transition time and why the initial prototype was slow.

It appears, however, that there are no examples of their use in electrochromic displays with moving-image speeds (24 frames/s; switching times of $<40 \mathrm{~ms}$ ). This is due to the fact that the color-switching rate of PEDOT is limited by the diffusion rate of counter- ions into the film during the redox process. The diffusion time, $\mathrm{t}$, of ions required to reach a saturation concentration in a polymer film, that implies switching time, is proportional to the square of films thickness, $x: t a x^{2} / D$, where $D$ is the diffusion coefficient of an ion in a polymer film. Therefore the simplest way to overcome the slow switching rates is to decrease the diffusion distance of ions, that is, to reduce film thickness. Based on the reported switching time of $2.2 \mathrm{~s}$ for a $300 \mathrm{~nm}$ thick PEDOT film, [15] we expect the switching time to be approximately $10 \mathrm{~ms}$ for a $20 \mathrm{~nm}$ thick film. However, 
the coloration of such a thin film is never sufficient for display applications.[14] Overall, diluted PEDOT material performed better than the original.

\subsubsection{Electrolyte Manufacturing and Optimization}

The objective of this section is to describe the formulation and the manufacturing behind the electrolyte. An off-the-shelf electrolyte from was used during the start of the display task and was intended to be used for the final iteration. Unfortunately, the company went out of business during the research and testing phase. As a result, different alternatives for substituting the electrolyte were researched. The option with the highest risk of failure was creating a new electrolyte formulation in the chemistry polymers lab. The reason the risk is relatively higher than the other options is because it involves some challenging chemical reaction setups to make the initial compounds needed before I could begin mixing electrolyte solutions with varying ratios and characterize the formulation. If the in-house made electrolyte did not function properly, the synthesized polymer material PMMA would have to be characterized before using it to make a second formulation. If the second formulation still not successful, it's would have been necessary to find a new way of synthesizing the compounds while testing the current formulation systems.

\subsubsection{Polymer Synthesis}

The polymer material that this electrolyte uses is poly(methyl methacrylate), PMMA. This material is synthesized via free radical 
polymerization and methyl methacrylate which is the monomer that was used. For any polymerization reaction, the reaction flask has to be sealed and with zero presence of oxygen. Therefore, the entire reaction has to be processed in a nitrogen environment. Figure 5.10 (a) and (b) are the images of vacuum setup using liquid nitrogen and reaction apparatus setup.

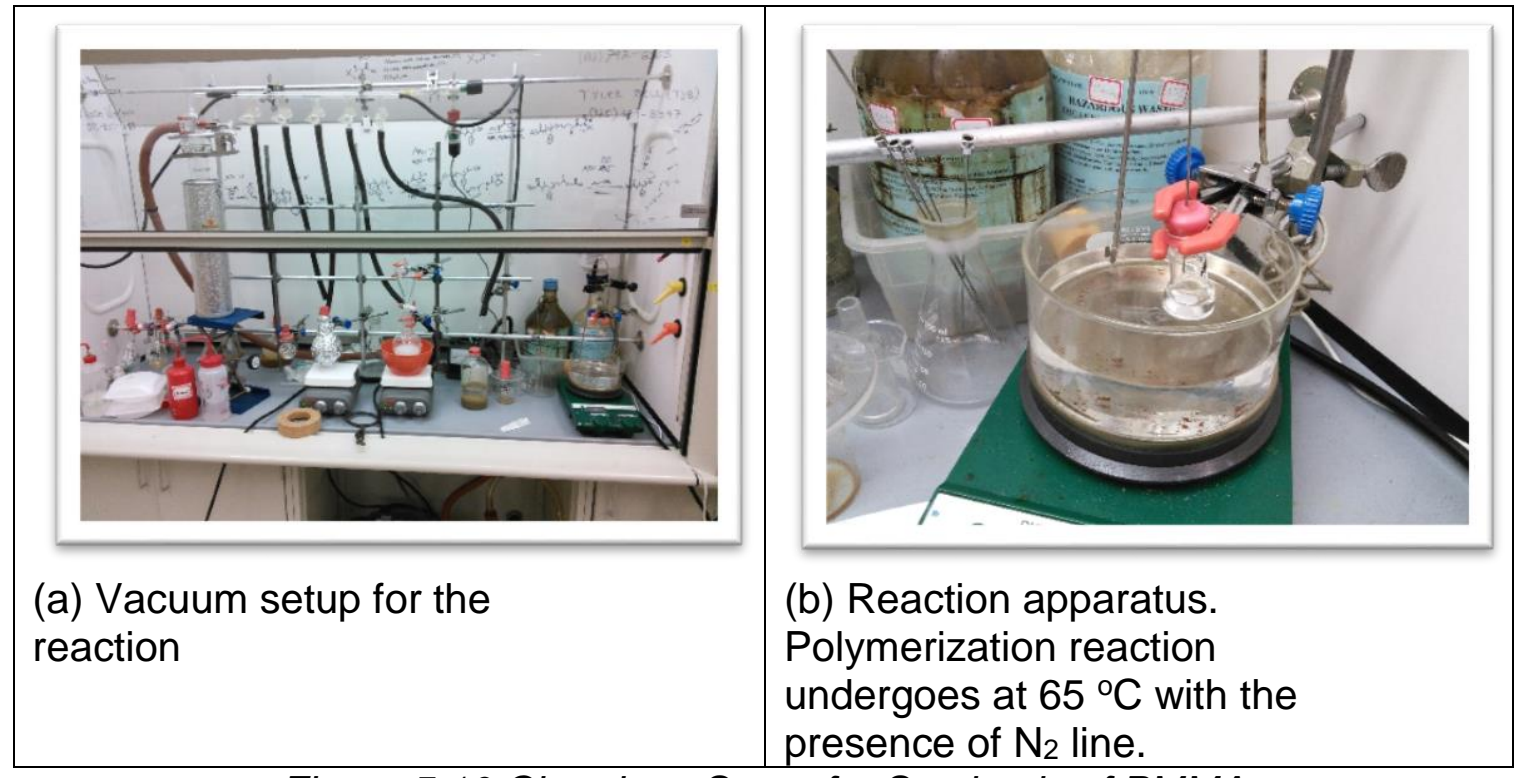

Figure 5.10 Chemistry Setup for Synthesis of PMMA

This polymerization reaction is initiated by AIBN initiator. The reaction flask needed to be submerged an oil bath for 3 hours at 65 degrees Celsius. The polymerization reaction was terminated after the 3 hours by introducing air into the flask and removing the heat source. The target polymer PMMA was precipitated out from the given solvent system by adding methanol. PMMA and methanol mixture were then vacuum filtered as shown in Figure 5.11. The PMMA was then dried in ambient conditions. 


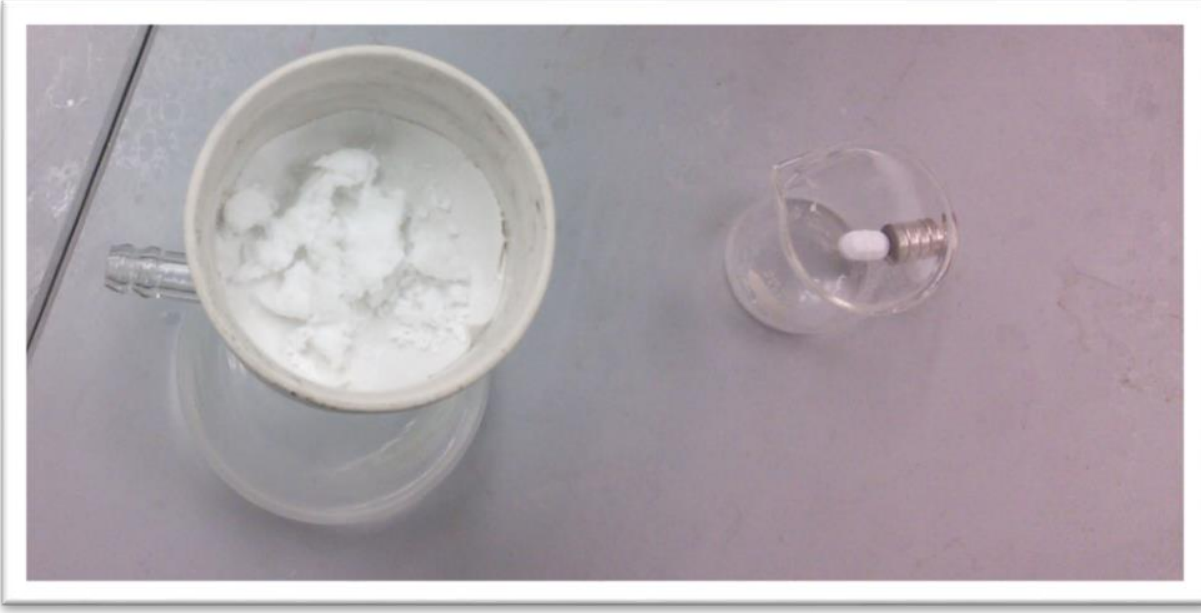

Figure 5.11 PMMA collected via vacuum filtration

\subsubsection{Mixture of Compounds}

After synthesis of the polymethyl methacrylate (PMMA), $15 \mathrm{wt} \%$ of it was mixed with $84.5 \mathrm{wt} \%$ of propylene carbonate. The solution was stirred and sonicated until the polymer was completely dissolved in the propylene carbonate. The final step was adding $0.5 \mathrm{wt} \%$ of Lithium Perchlorate $\left(\mathrm{LiClO}_{4}\right)$ to the solution. 


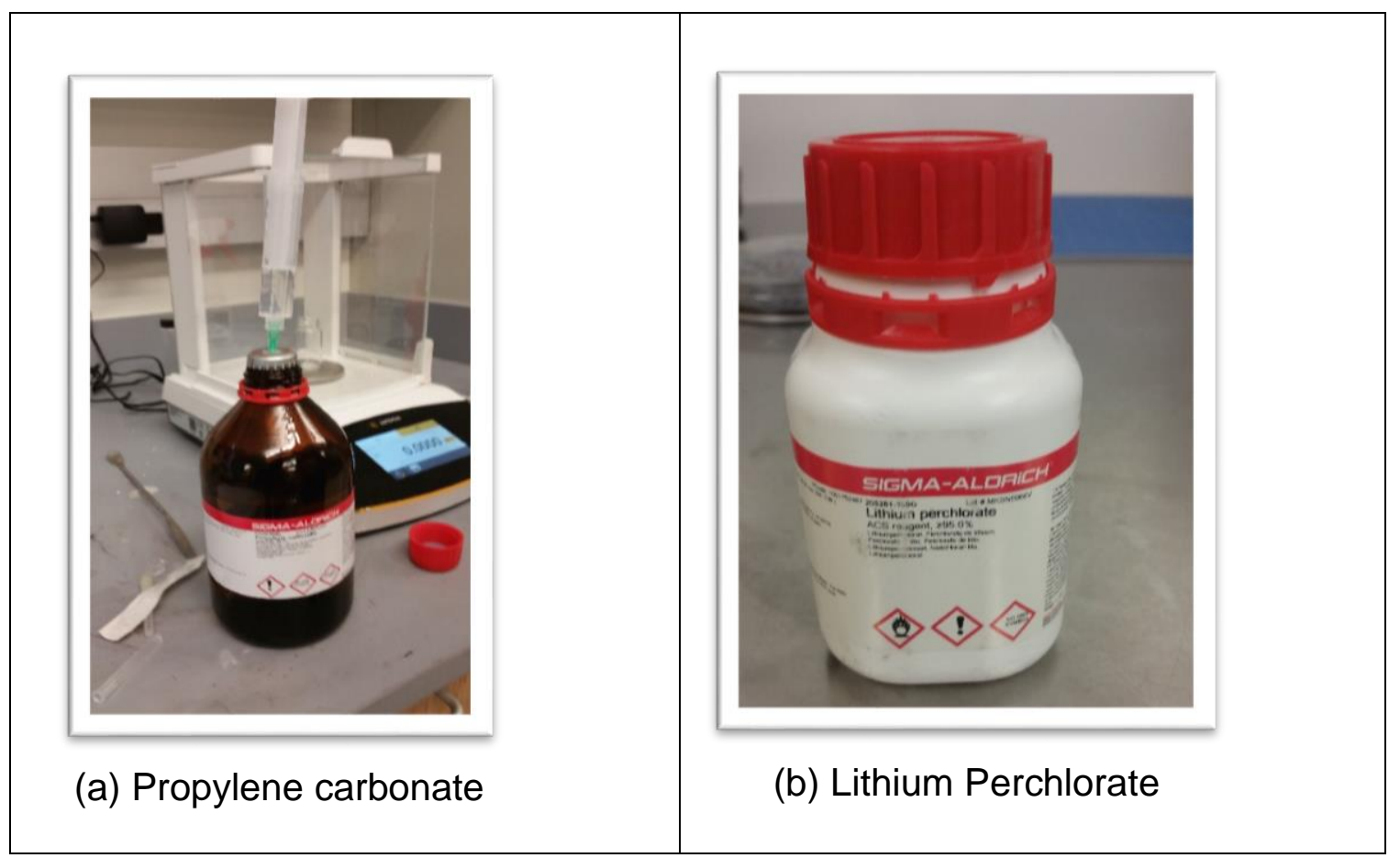

Figure 5.12 Compounds Needed for Electrolyte Mixture

The final result was a clear electrolyte shown in Figure 5.13.

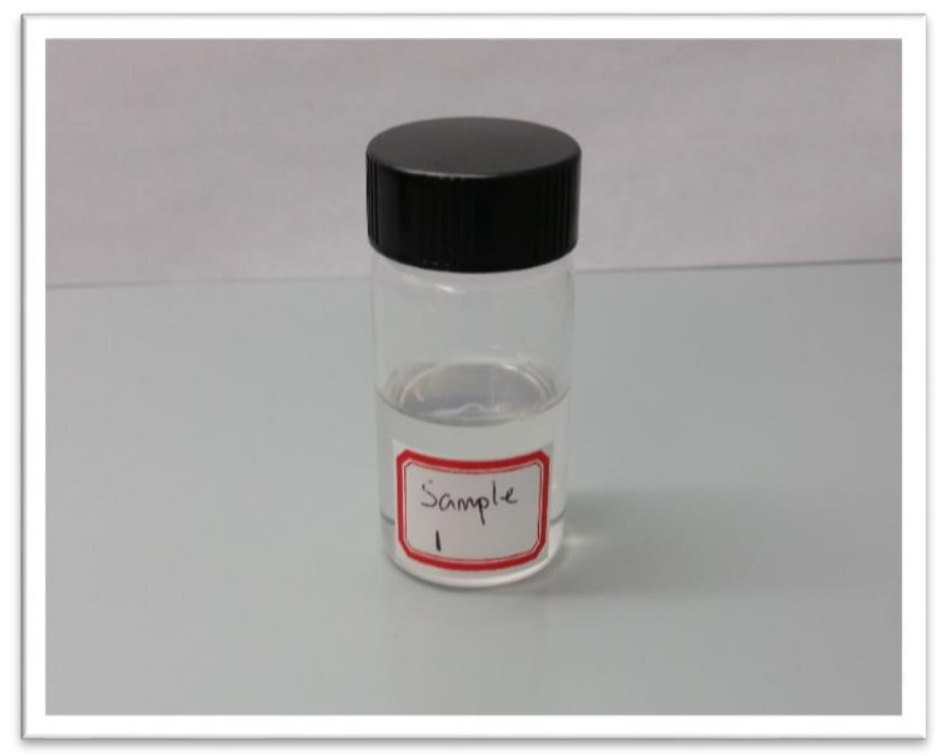

Figure 5.13 Final product of mixed compound polymer electrolyte

\subsubsection{Electrolyte Optimization Results}

The electrolyte was optimized varying ratios of Lithium Perchlorate

concentrations. Out of the 20 iterations, sample 1 had the best results of 
transition time from oxidation to reduction and vice versa. Leakage current was minimal in the sample 1 display which verified the state hold time and there seemed to be a relation between the concentration of ions and transition time. However, the concentration has its limits and once this limit is violated the opposite effect occurs, leakage current increases and transition time decreases.

\subsubsection{Printing Technology Process for Display}

The display was designed small enough to fit on average size contact lens. In order to implement such a small design a fabrication process was needed for suitable manufacturing. Dr. Malcolm Kief, from the Graphic Communications Department, helped in teaching screen printing techniques which were used to fabricate the display. With the added benefits of screen printing we were able to achieve quick turnaround for prototypes, testing, and alterations in the design that were deemed necessary for a well-functioning device.

The overall process of screen printing used for placing material onto a flexible and transparent substrates can be summed up in 3 steps. This is illustrated in Figure 5.14 where a pattern screen sits above the substrate and material ink is placed on top of the screen. The ink is push evenly across the pattern on the screen with the flood bar this step is known as flooding. The next step is having the squeegee press against the pattern of the screen in the opposite direction. The pressure from the squeegee forces the ink through the gaps of the screen onto the substrate as it passes across the screen. The final step is flooding the screen once more to maximize the material placement onto the substrate. 


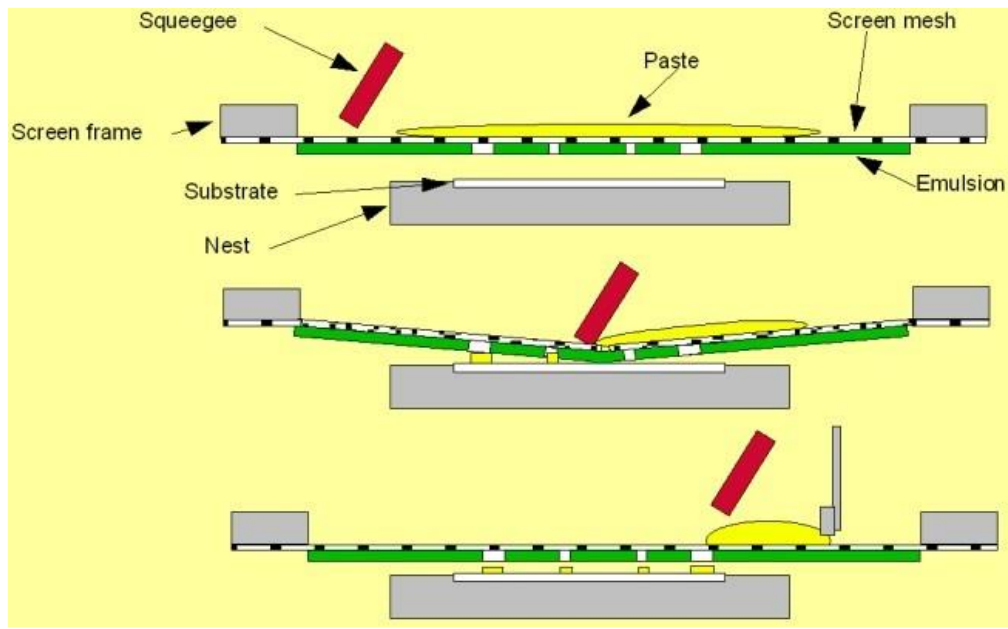

Figure 5.14 Screen Printing Illustration

Next I would like to describe the steps for making a screen. The first step in making a patterned screen is designing the pattern in CAD to make a polarity mask. This mask acts as a film positive which allows the design to transfer onto a type of ultraviolet (UV) sensitive photoresist material known as emulsion. The patterned sheet initially is sheet of carbon which is shown in figure 5.15 .

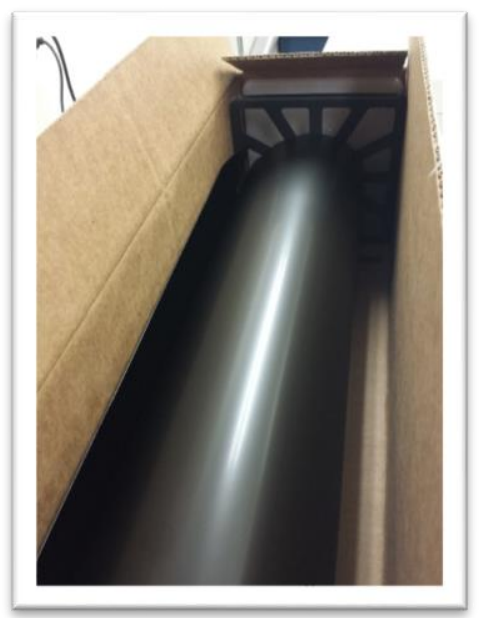

Figure 5.15 Carbon Sheet Roll

The roll of carbon was cut into a sheet that was then inserted into the Cyrel

Digital Imager. The imager is equipped with a fiber laser that will remove the 
excess carbon on sheet when the CAD file is uploaded. The final result is a carbon composite of the design.

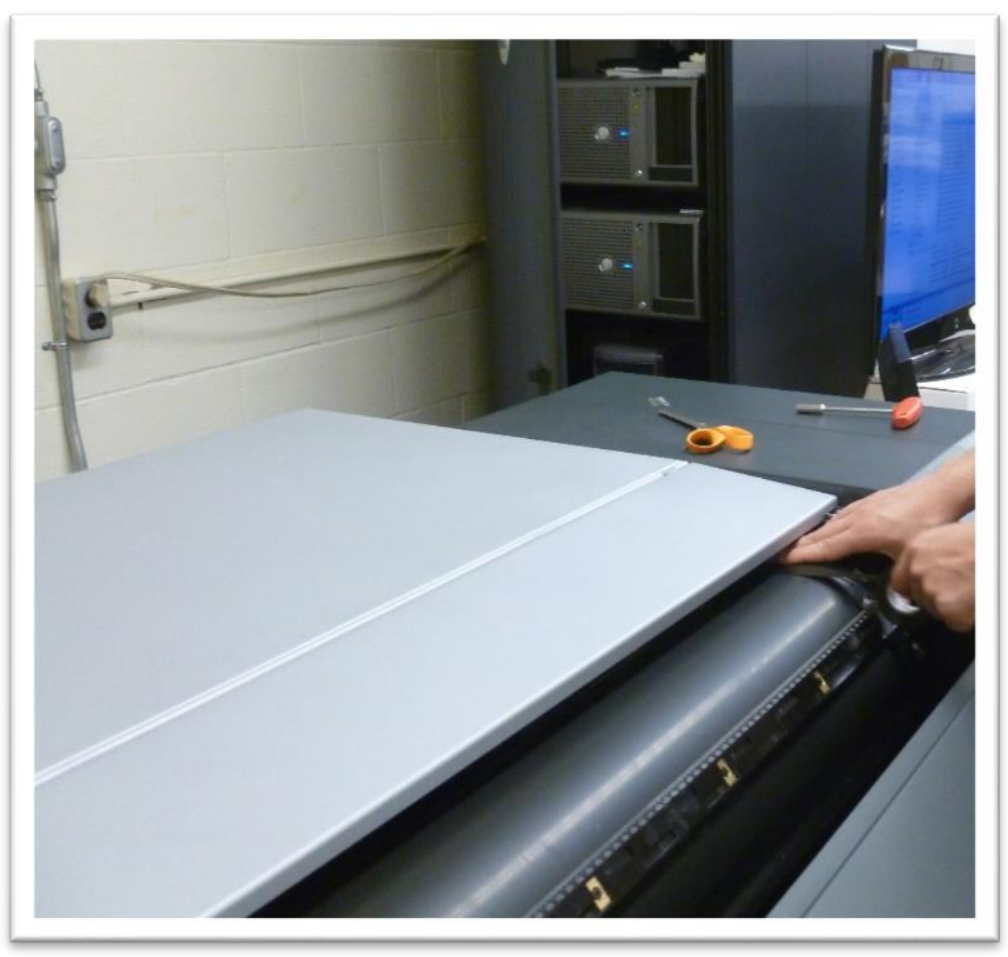

Figure 5.16 Feeding Carbon Sheet into Cyrel Digital Imager 


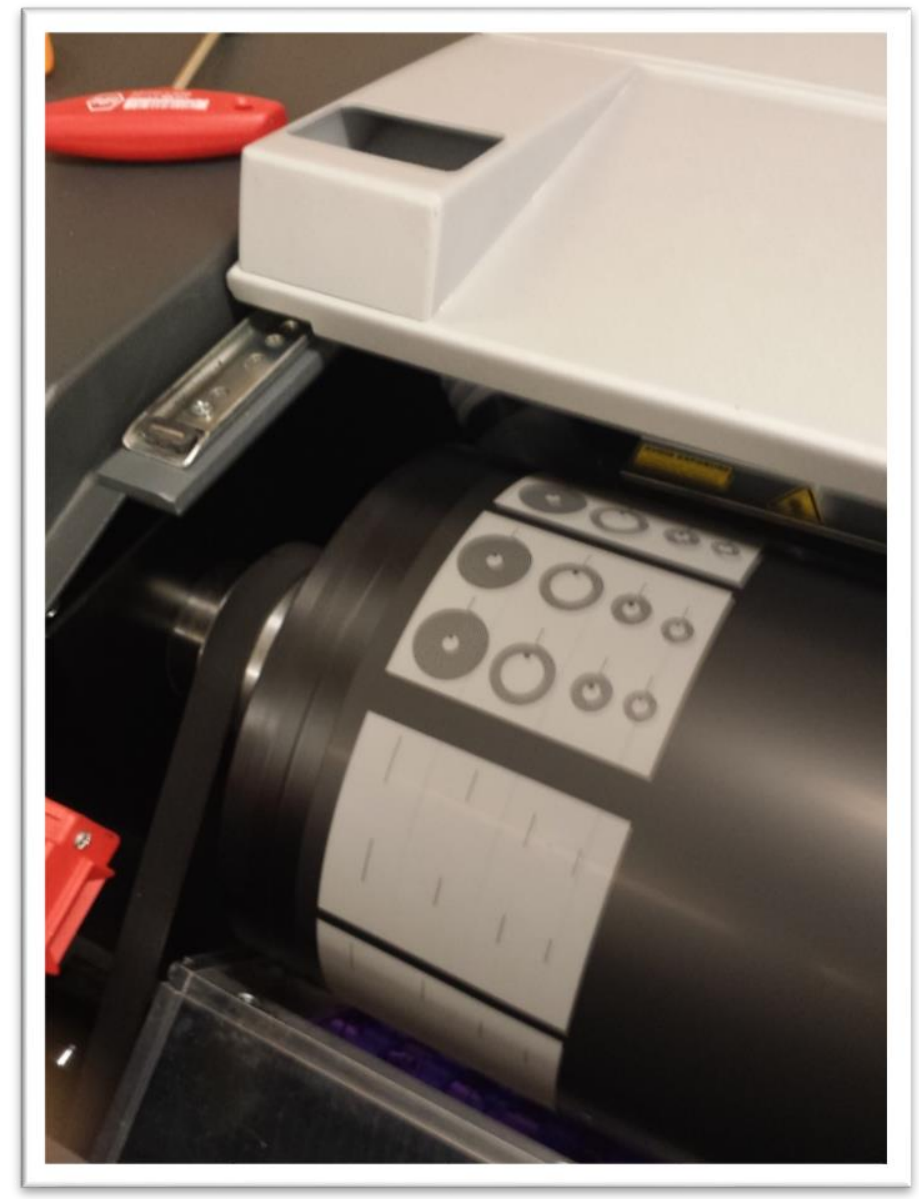

Figure 5.17 Resulting Mask

Once the mask was made, the design needed to be transferred to the screen.

A screen can consist of an array of fine polyester fibers threads that are attached across an aluminum frame. These fibers are cross linked at a specific distance which is determined by the thread count rating; this is known as the mesh number. The mesh number correlates to the number of threads in a square inch. The mesh number used for the silver traces on the initial prototypes were number 160 and later up graded to the 460 in the final version. 


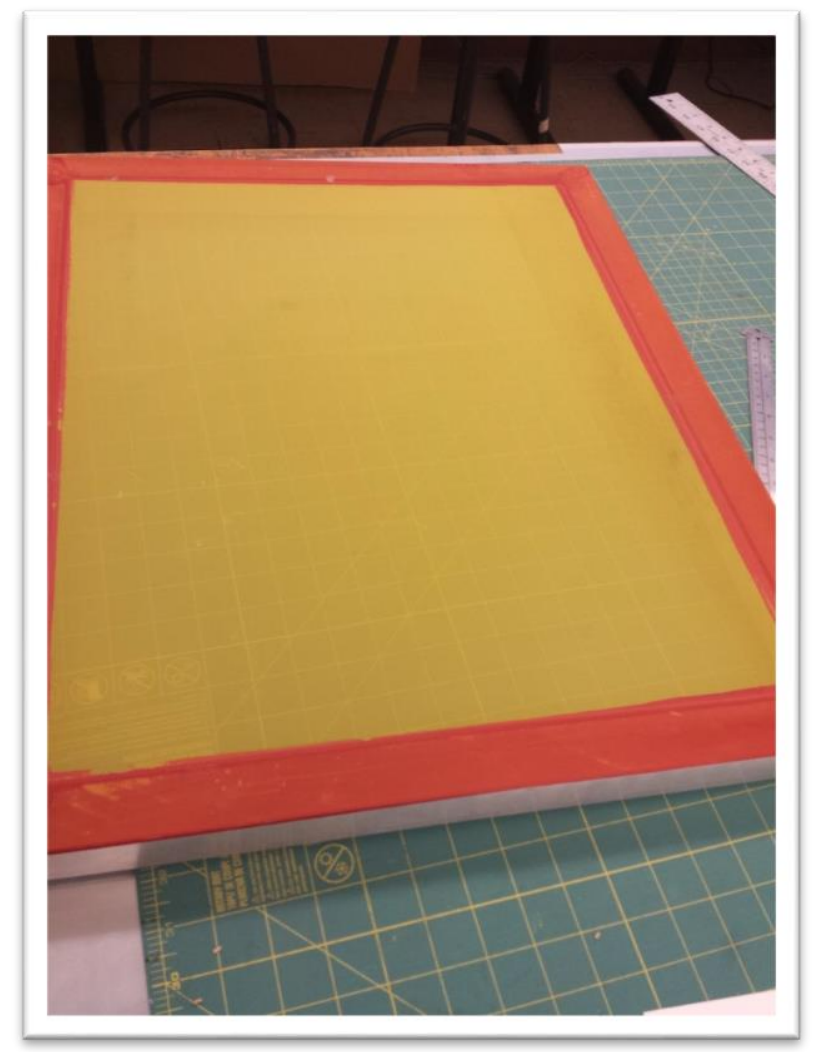

Figure 5.18 Screen

To create a stencil of the desired design in screen, emulsion needed to be placed on the screen. Figure 5.19 below shows all the tools need to apply emulsion onto a screen. 


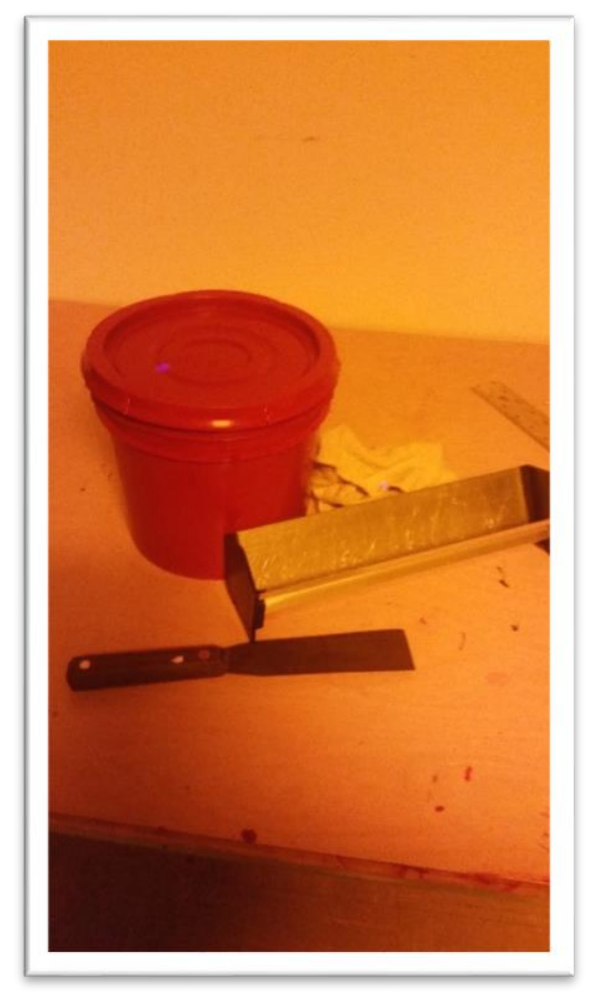

Figure 5.19 Emulsion Placement Tools

The screen was anchored to a sturdy platform the emulsion was poured in a vshaped tool that would swipe across the screen depositing the thin uniform layer on the screen. This step was repeated 3 times on the front side of the screen and 2 times on the back side of the screen. Note that these step had to be performed in a dark room where UV light was filtered out. Since the emulsion is a UV-sensitive polymer exposer to that specific range of wavelength will cause the emulsion to harden and make it insoluble to water. The emulsion was placed in a screen drying cabinet at 80 degrees Fahrenheit to optimize drying time. If the screen was air dried it would take 24 hours to ensure that the emulsion was completely dry. 


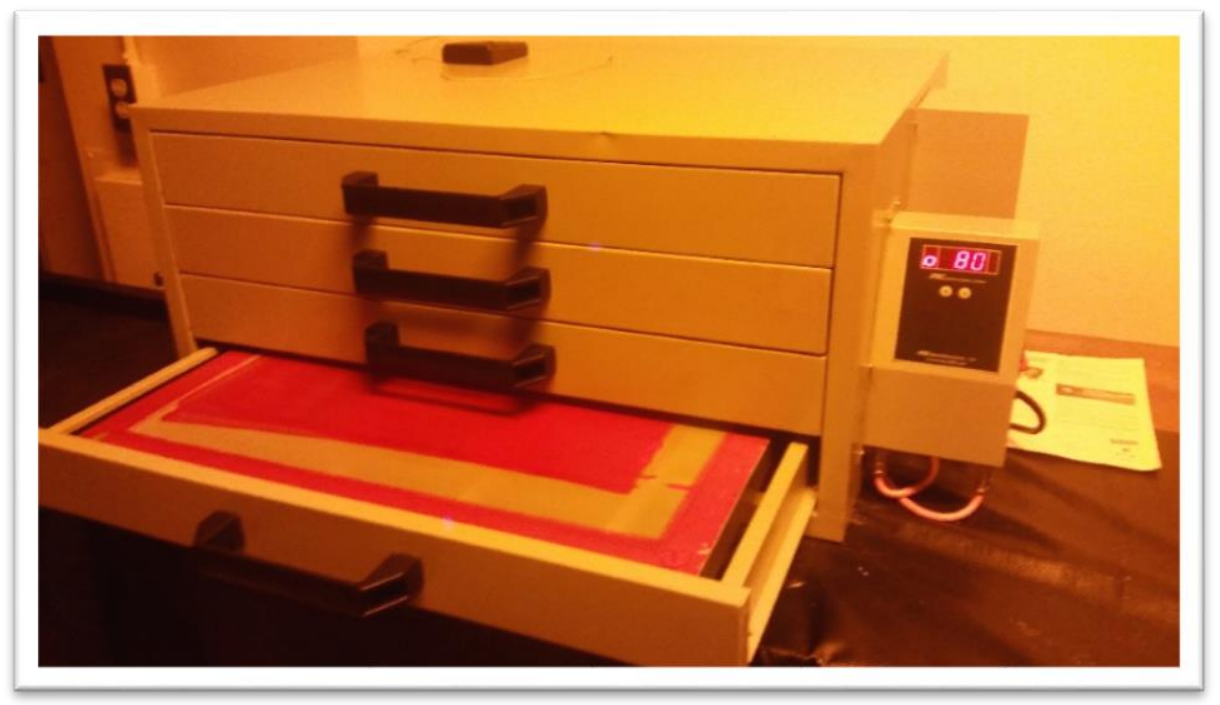

Figure 5.20 Screen Drying Cabinet

Once dry the mask is placed onto of the screen shown in Figure 5.21. The purpose of the mask is to control the pathway of UV light and prevent exposure on the location of the pattern. This is how the design is transferred onto the emulsion and creates a stencil.

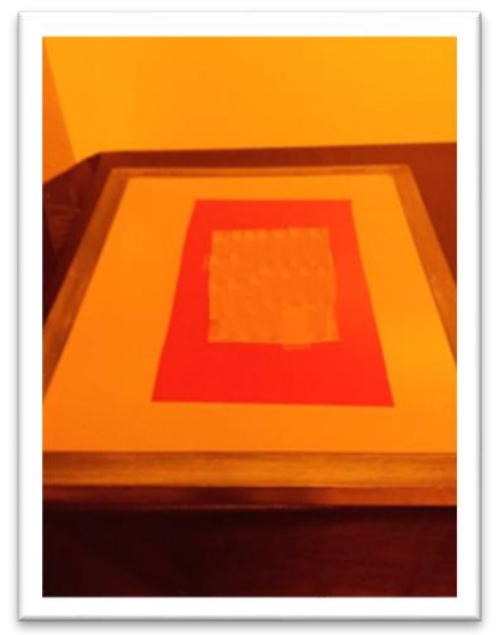

Figure 5.21 Mask Laid On Top of Emulsion

The screen with the mask was placed in a UV curing machine for exposing. This step is similar to photolithography techniques where too little exposure will cause features to blowout during the wash process or completely wash away the entire 
emulsion when developed and too much exposure will cause undercutting. It was determined that the emulsion should be exposed with a light intensity of 120LTU in order to achieve sufficient mask feature size for printing the display.

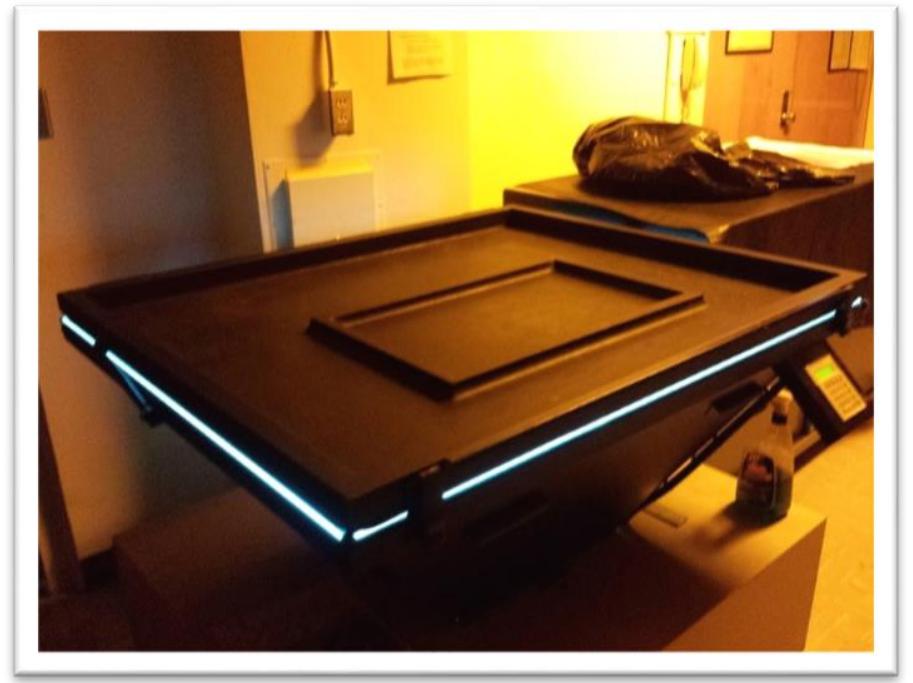

Figure 5.22 Screen in UV Curing Machine

After exposing the emulsion for 2 minutes, the screen was gently washed of in the hydrobooth where the unexposed parts are washed off. The screen is placed in reference to the hooks in the booth, with the backlight off, and soaked with water for approximately 1 minute. After 1 minute the backlight is turned on and addition spraying is used on the patterned areas. 


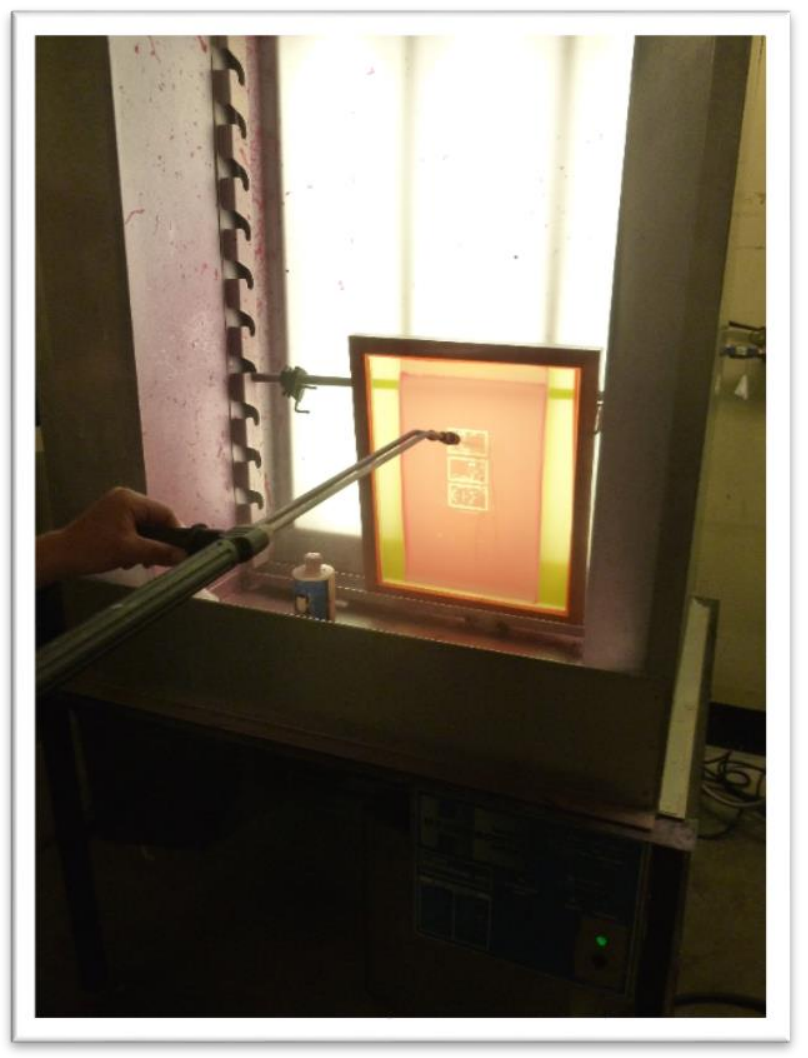

Figure 5.23 Washing Screen

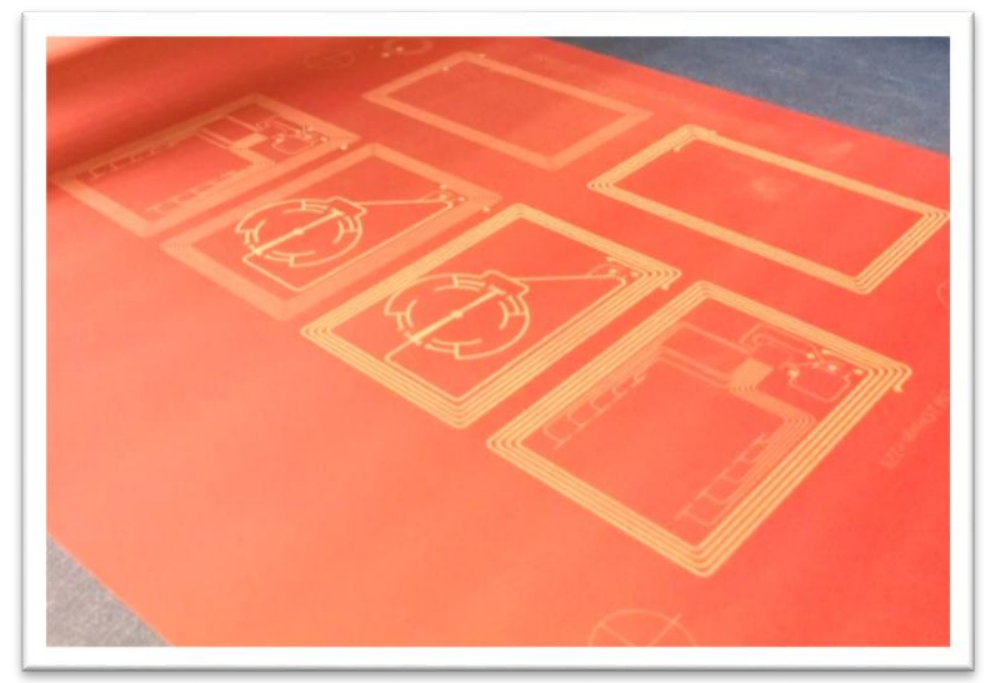

Figure 5.24 Final Stencil Design

Once the screen has been washed and dried, a second post-exposure at $120 \mathrm{LTU}$ was performed to harden the emulsion and increase durability. At this point the screen is finished and ready for printing. 
For printing the substrate a $25 \mu \mathrm{m}$ Mylar PET film from DuPont was used. PET has been used in printed electronics for years this is how we were able to choose a thin film substrate with an ink absorbing coating. The machine used for printing the display was the ATMA AT-45FA Pneumatic Screen Printer. The screen that was manufactured in the previous section was placed in the brackets of the machine. A flood bar and squeegee were also attached to the machine. The silver ink was placed on the screen shown in Figure 5.25.

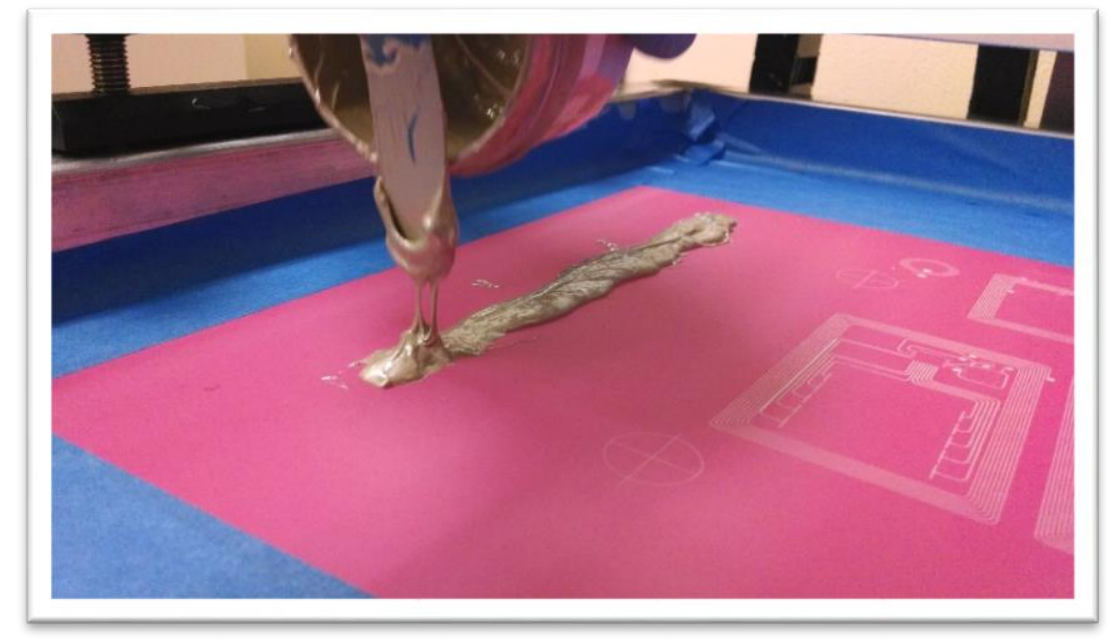

Figure 5.25 Applying Silver Ink Paste

Next, the entire area of the screen is coated with a thin layer of ink known as flooding. By initially flooding the screen presents the design with an even layer of ink for consistent deposition across the print. The flood bar sweeping the ink across the screen from left to right with minimal pressure shown in Figure 5.26. 


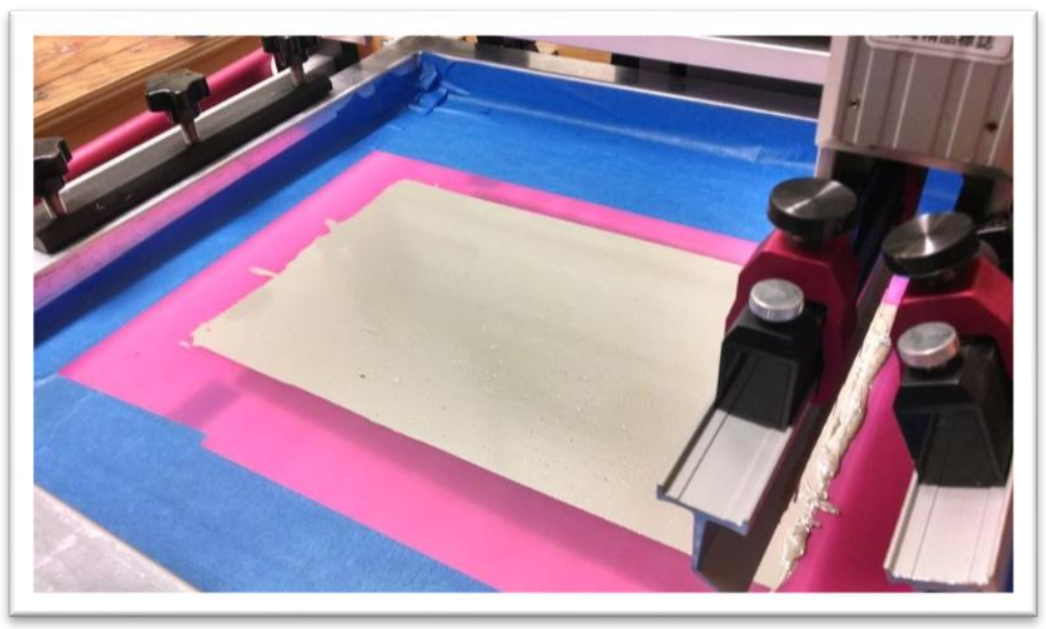

Figure 5.26 Flooding the Screen

Once the screen has been flooded, the squeegee was applied and pushed the ink onto the substrate going the opposite direction of the flooding step. Finally, the screen is re-flooded to prevent the ink from drying and clogging the mesh. The final result of the print is shown in Figure 5.27.

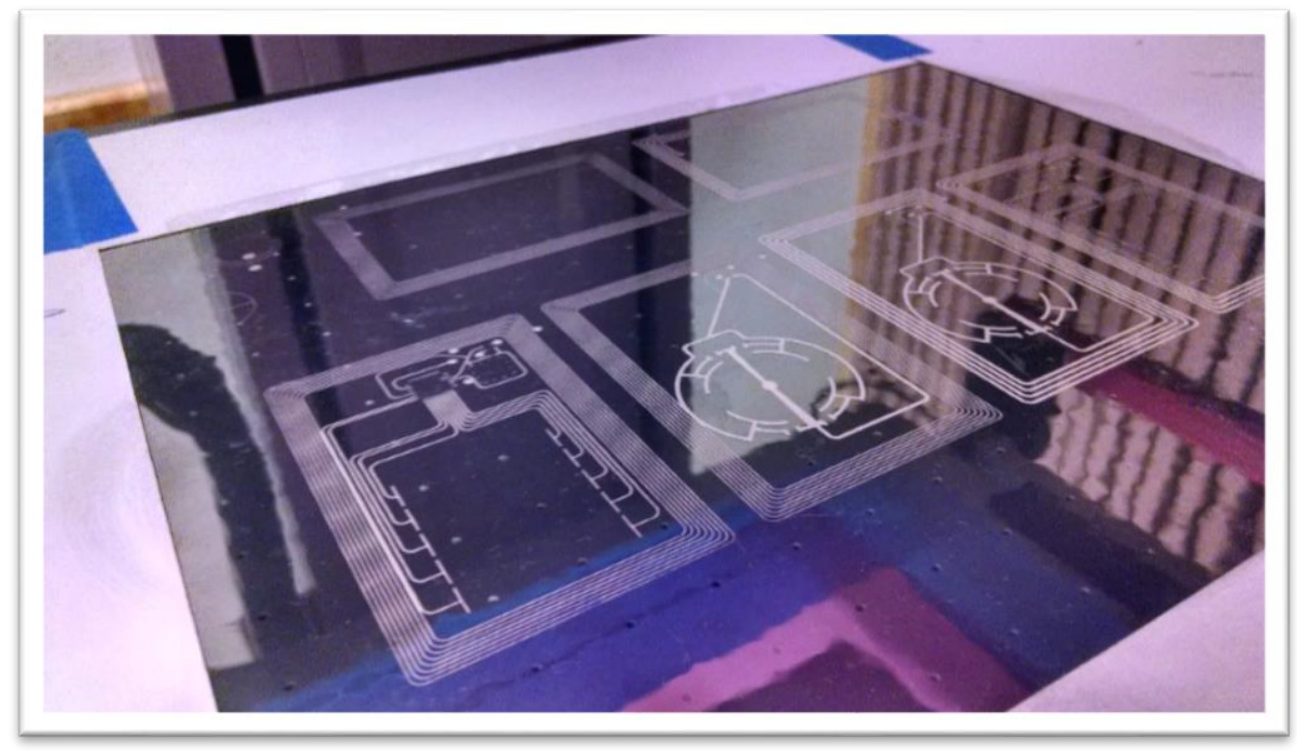

Figure 5.27 Printing Result with Silver Ink

The same steps were taken for placing the PEDOT:PSS and electrolyte. Figure 5.28 shows the PEDOT and electrolyte material used. The final product of the initial prototype is shown below in Figure 5.29. 


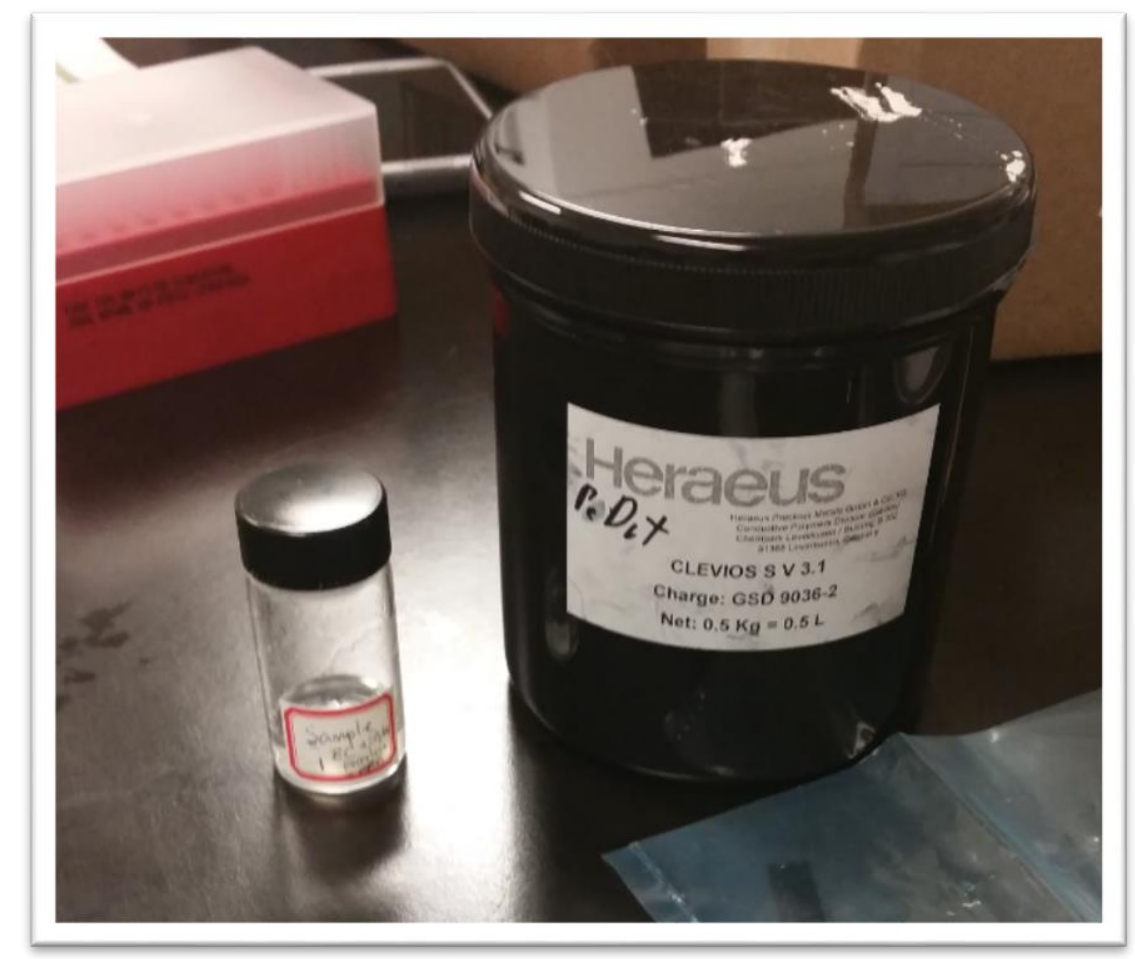

Figure 5.28 PEDOT:PSS and Electrolyte Material

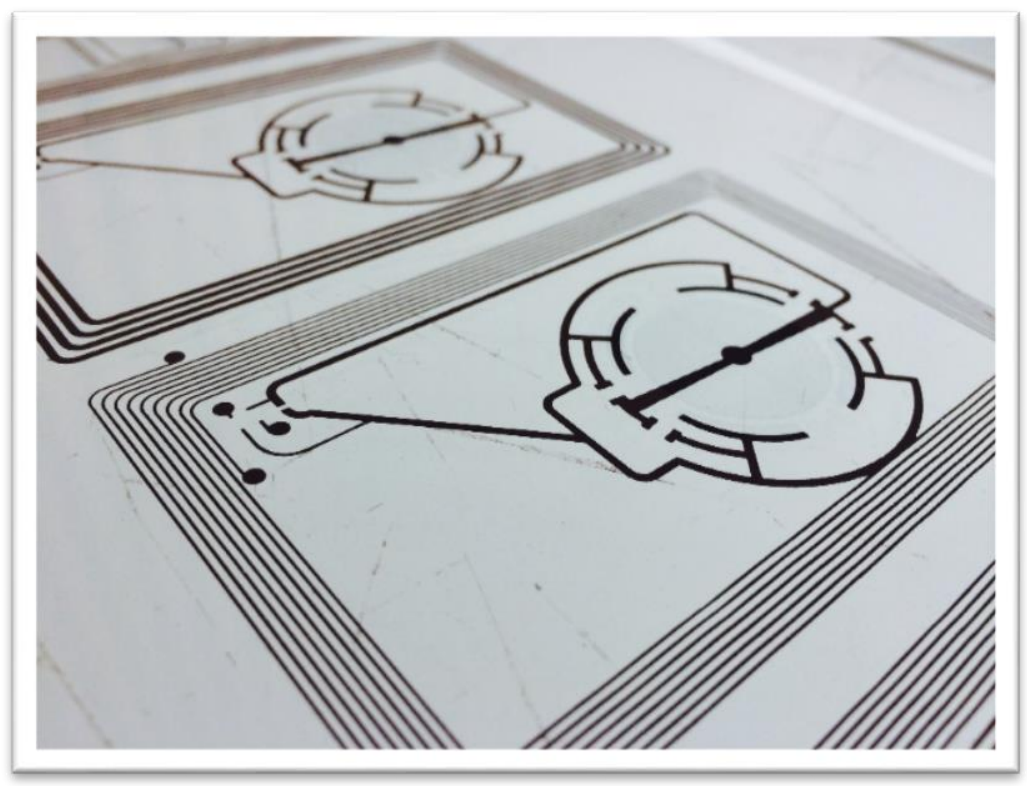

Figure 5.29 Final Print of Silver Ink, PEDOT:PSS, and Electrolyte

Finally, a 1 volt potential was used to test the functionality of the device.

The results can be seen in Figure 5.30. 


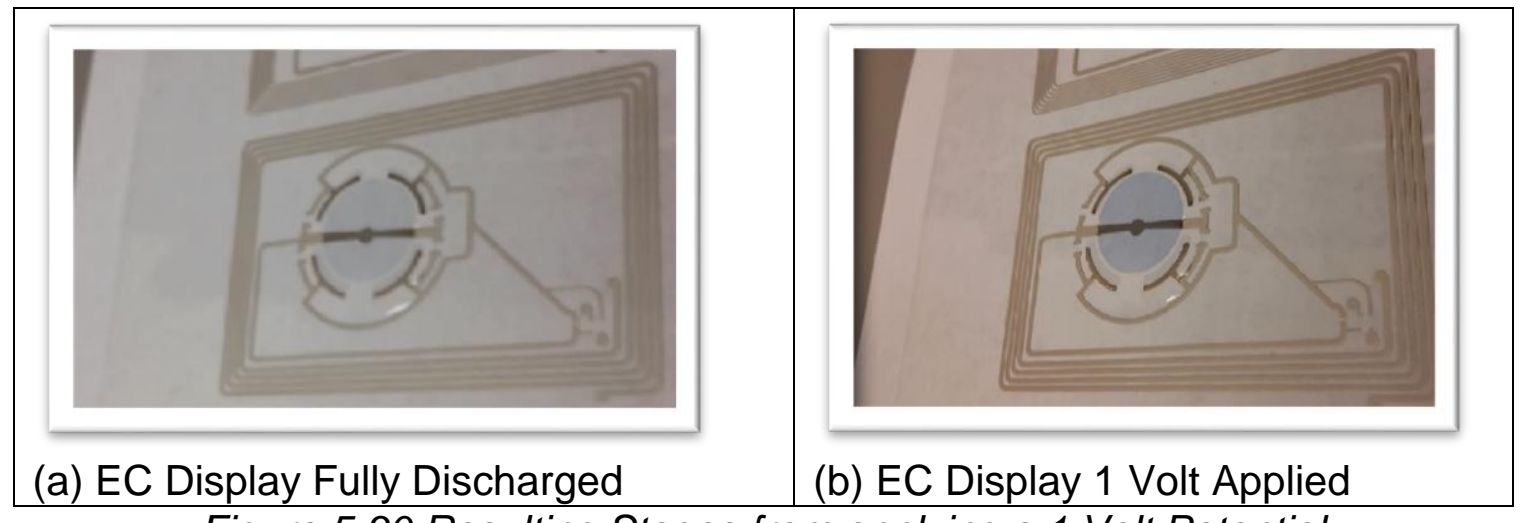
Figure 5.30 Resulting Stages from applying a 1 Volt Potential 


\section{Chapter 6}

\subsection{Encapsulation}

Once the entire system has been embedded into one substrate, the entire system needs to be encapsulated in a material that has the following properties: biocompatibility, clarity, durability, and comfort. The final prototype lens was a hybrid of a PET based substrate for the system's circuitry and PDMS encapsulation layers for both sides of the PET shown in Figure 6.1.

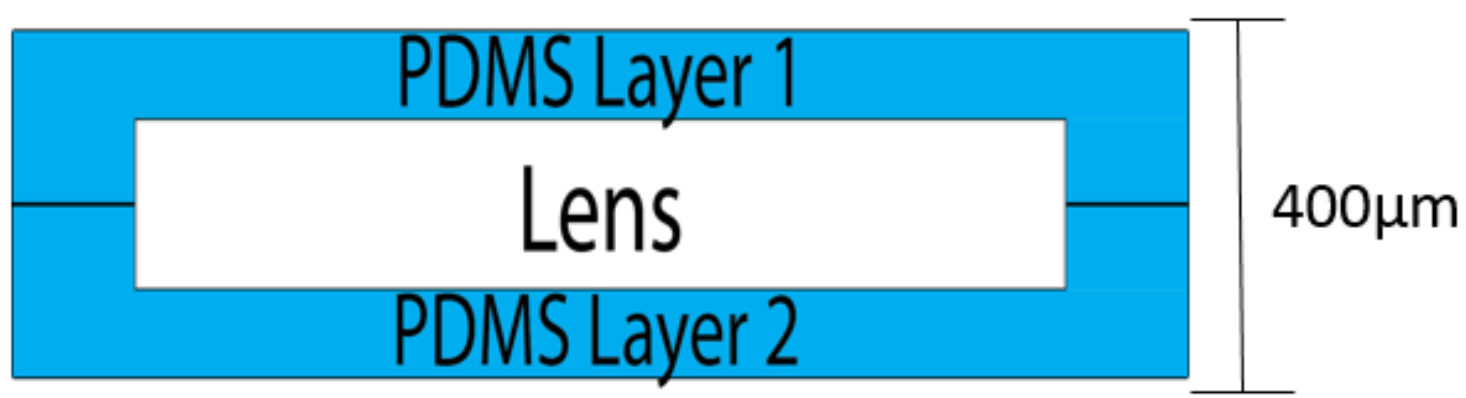

Figure 6.1 PET lens base completely encapsulated by PDMS Curving of the PET lens proved to be a much easier process than expected, however it still had its challenged. Wrinkle formation was a problem, as many times the edges of the antenna would have a couple of folds toward the outer rim of the lens. This was due to the material properties of the PDMS not chemically binding to the PET. From multiple trials including surface manipulation with plasma bonding machine it would not stick to the PET. However, even with this challenge, the center of the lens usually stayed free from wrinkles. We were able to minimize the wrinkles by trimming the edges of the PET without damaging the antenna. The result is shown below in Figure 6.2. 


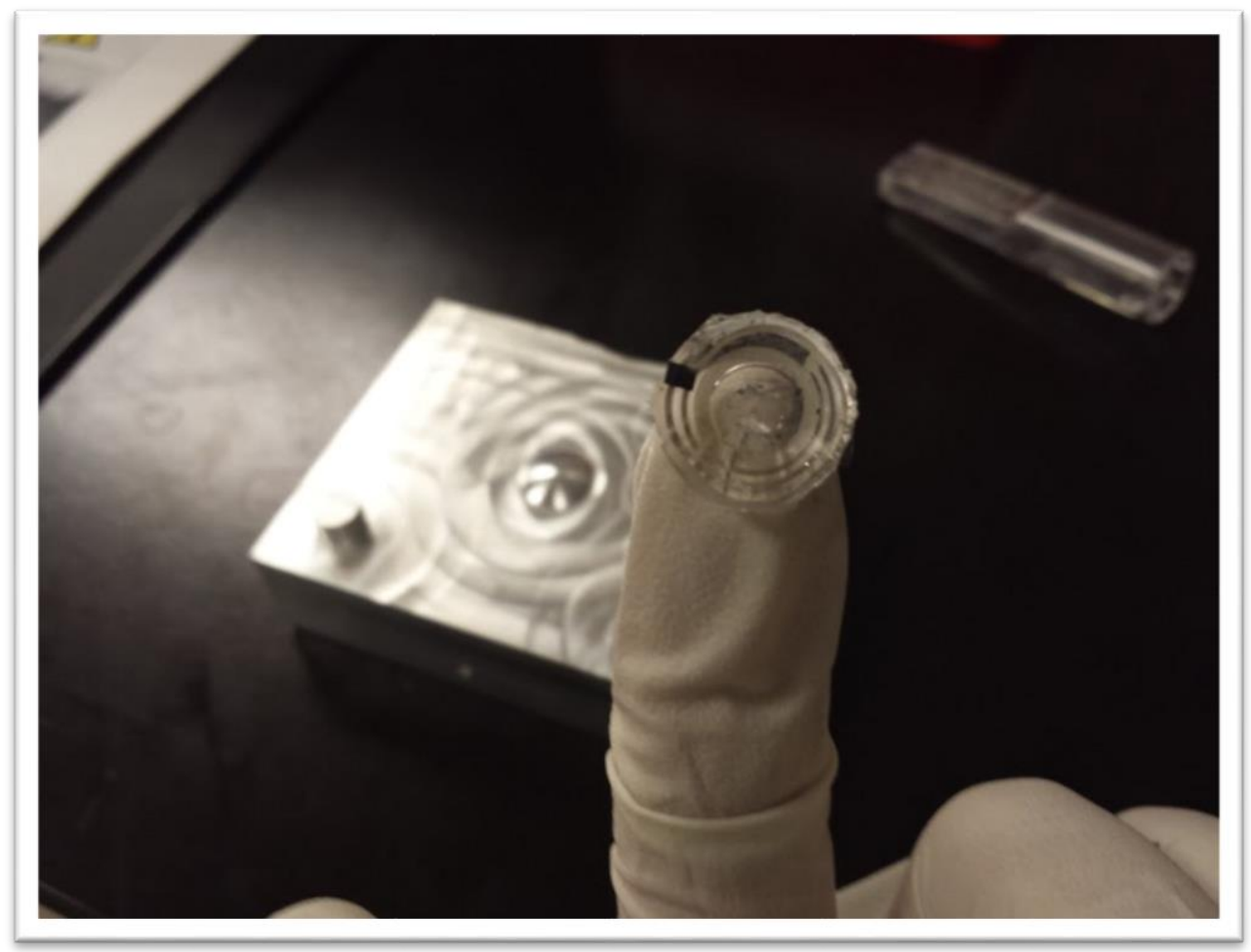

Figure 6.2 Encapsulated Contact Lens

\subsection{Encapsulation Procedures}

The process overview of encapsulation involves four major steps: PDMS manufacturing, Attaching PET substrates to Mold 1, use of Mold 2 for building top layer of PDMS, and use of Mold 3 to build bottom layer of PDMS. To carry out this process, manufacturing techniques from microfabrication, package manufacturing, and machining were used.

\subsubsection{PDMS Manufacturing}

Poly(dimethylsiloxane), also known as PDMS, is a synthetic polymer (silicone rubber) that has a Si-O-Si backbone with each Si atom having two methyl groups $(\mathrm{CH} 3)$. The form used for this research was a crosslinked PDMS which can be cured at room temperature or at elevated 
temperatures. PDMS possesses a wide range of properties that make it applicable for use as a material for micro scale devices. [16] The total quantity was determined based on the volume of the needed to fill the all three molds. It is difficult however to create a $5 \mathrm{~mL}$ of PDMS with the given equipment therefore $55 \mathrm{~mL}$ batch was made and multiple iterations of molding were implemented to determine success and optimization in processing. The PDMS mixture consist of a base and curing agent that is mixed in a 10:1 ratio.

For the $55 \mathrm{~mL}$ the mixture consisted of $50 \mathrm{~mL}$ of base solution and $5 \mathrm{~mL}$ of curing agent.

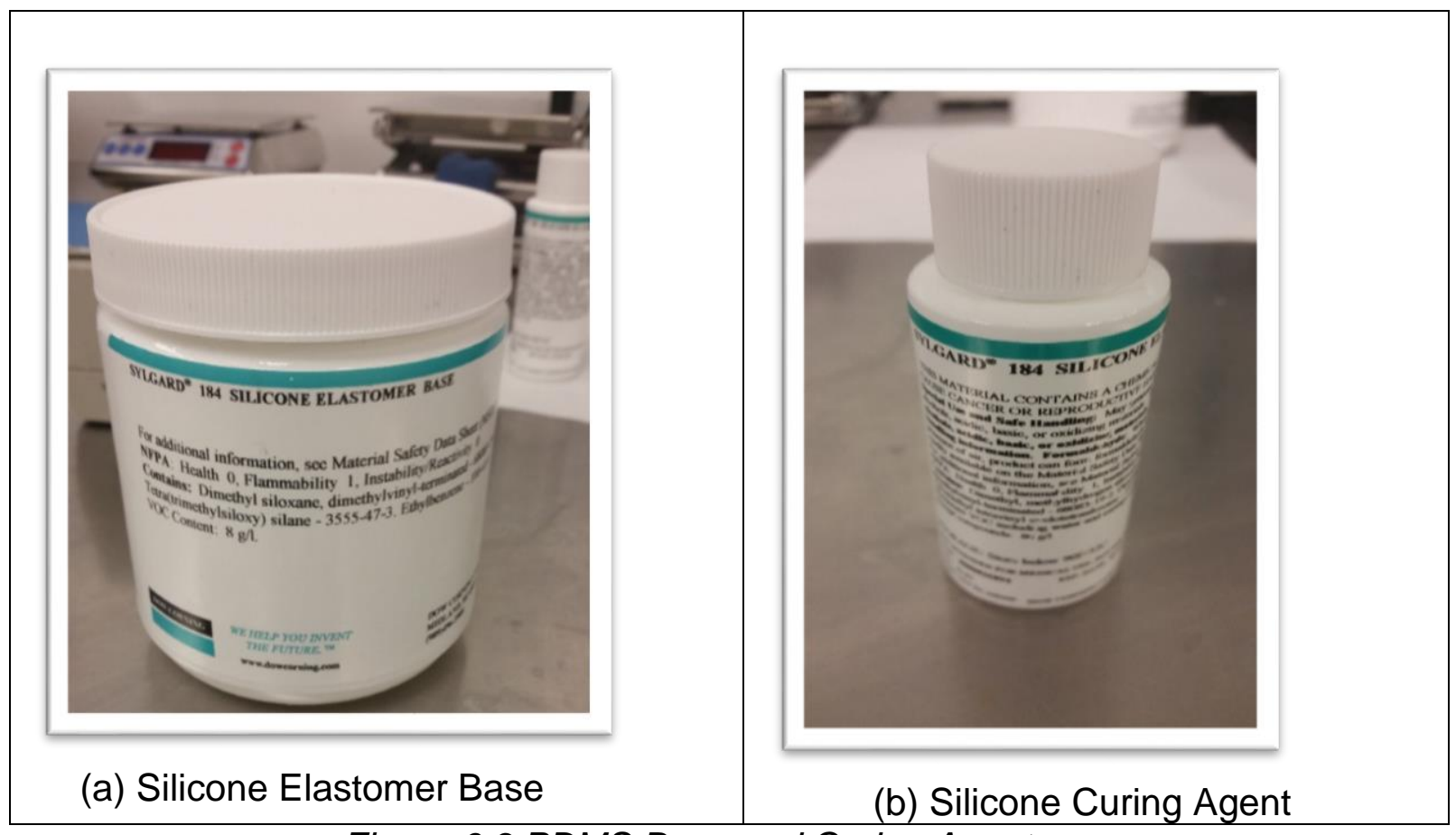

Figure 6.3 PDMS Base and Curing Agent

Using available syringes, the PDMS components were dispensed into a plastic mixing/measuring cup. The components were then stirred 
thoroughly and slowly for several minutes to avoid introducing excess oxygen into the mixture.

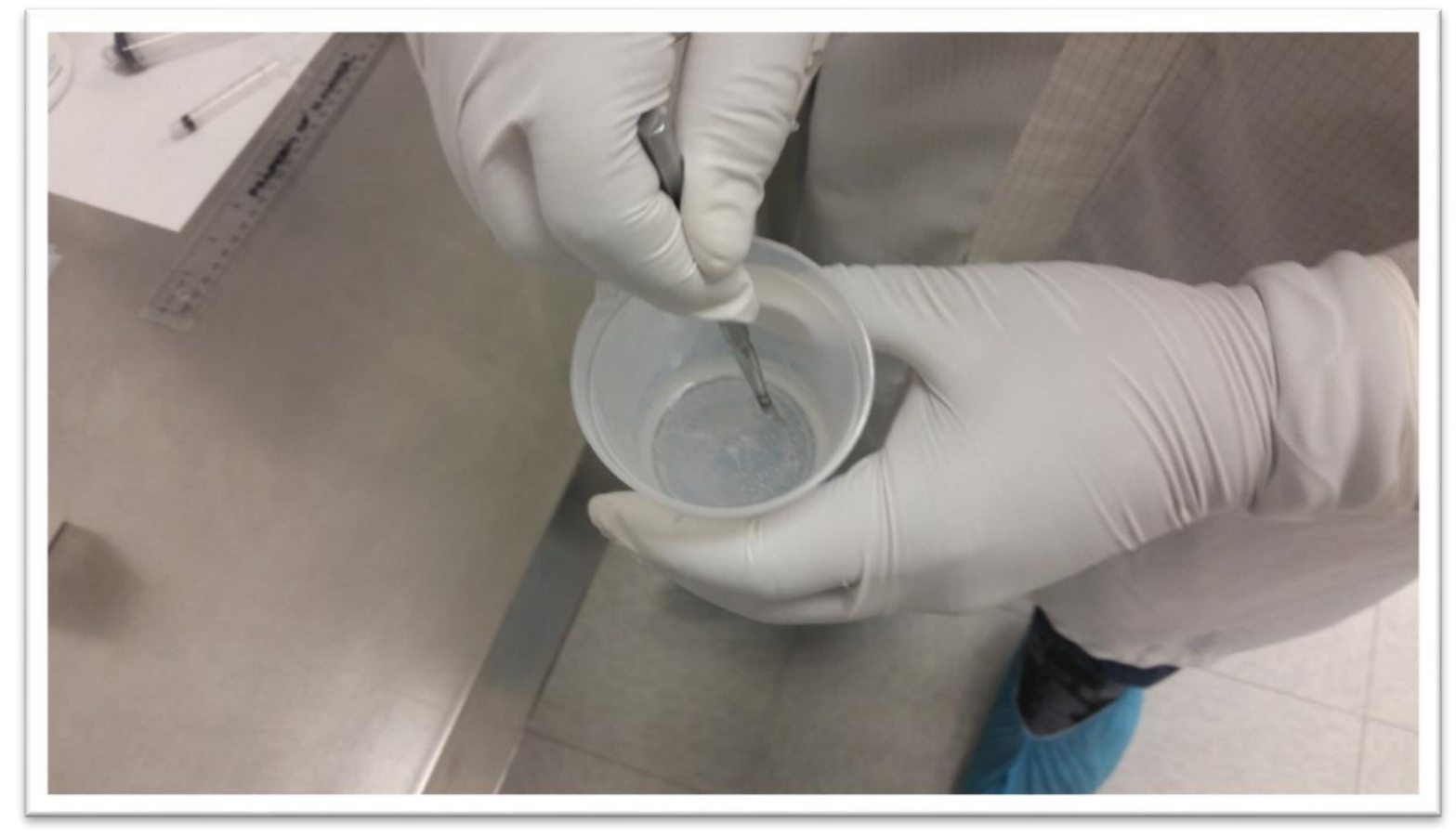

Figure 6.4 PDMS Solution Mixture in Cup

The next step in the process is to place the PDMS in a vacuum chamber for degassing. With vent valve and vacuum line valve closed, the vacuum pump is turned on from the utility chase. 


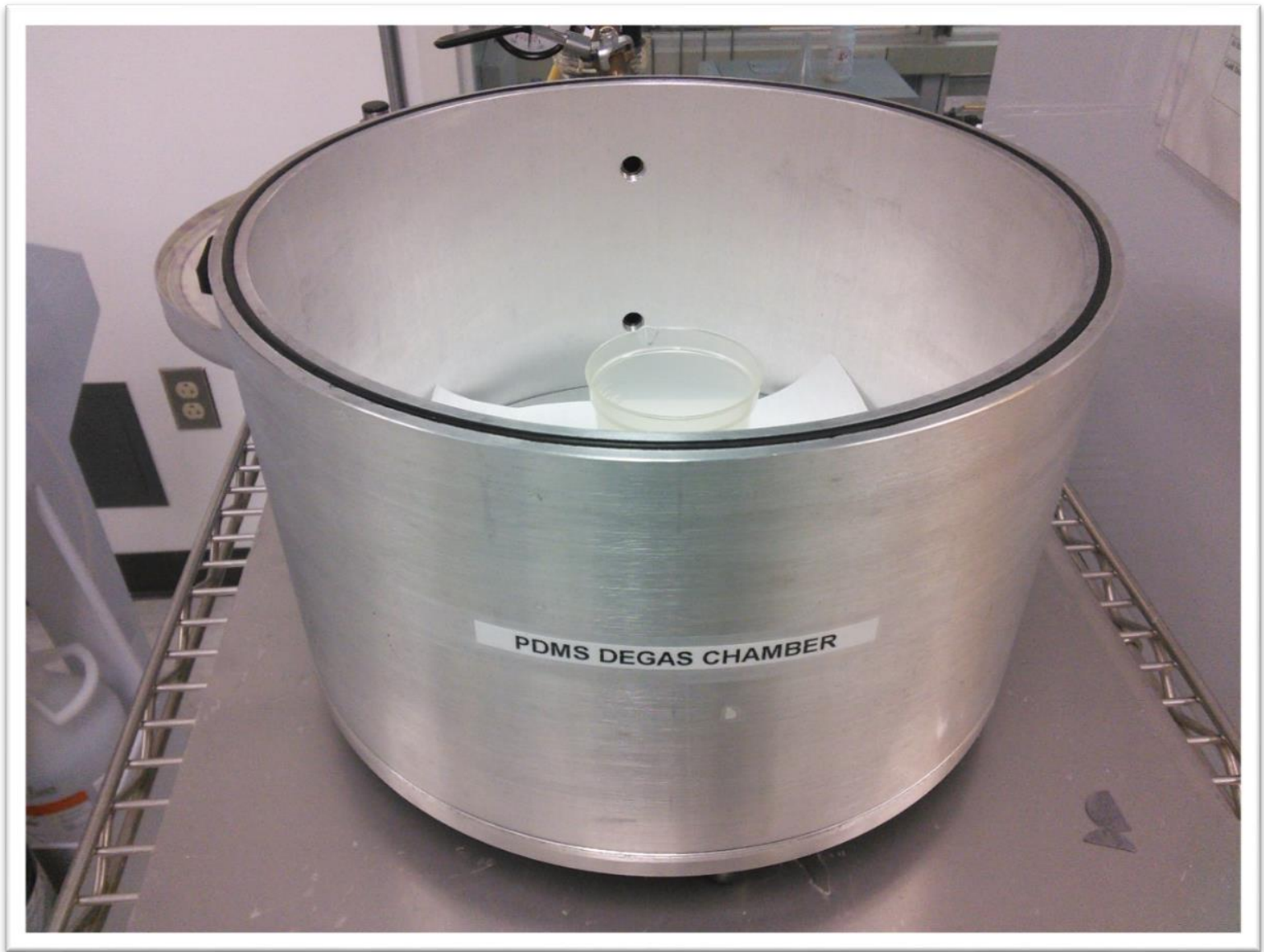

Figure 6.5 Mixture Inserted in Degas Chamber

The vacuum line valve is then opened and chamber pumps down until large bubbles come out of PDMS. During the pump down step it is a good idea to throttle chamber pressure using the vent valve so that PDMS doesn't overflow cup in the chamber. 


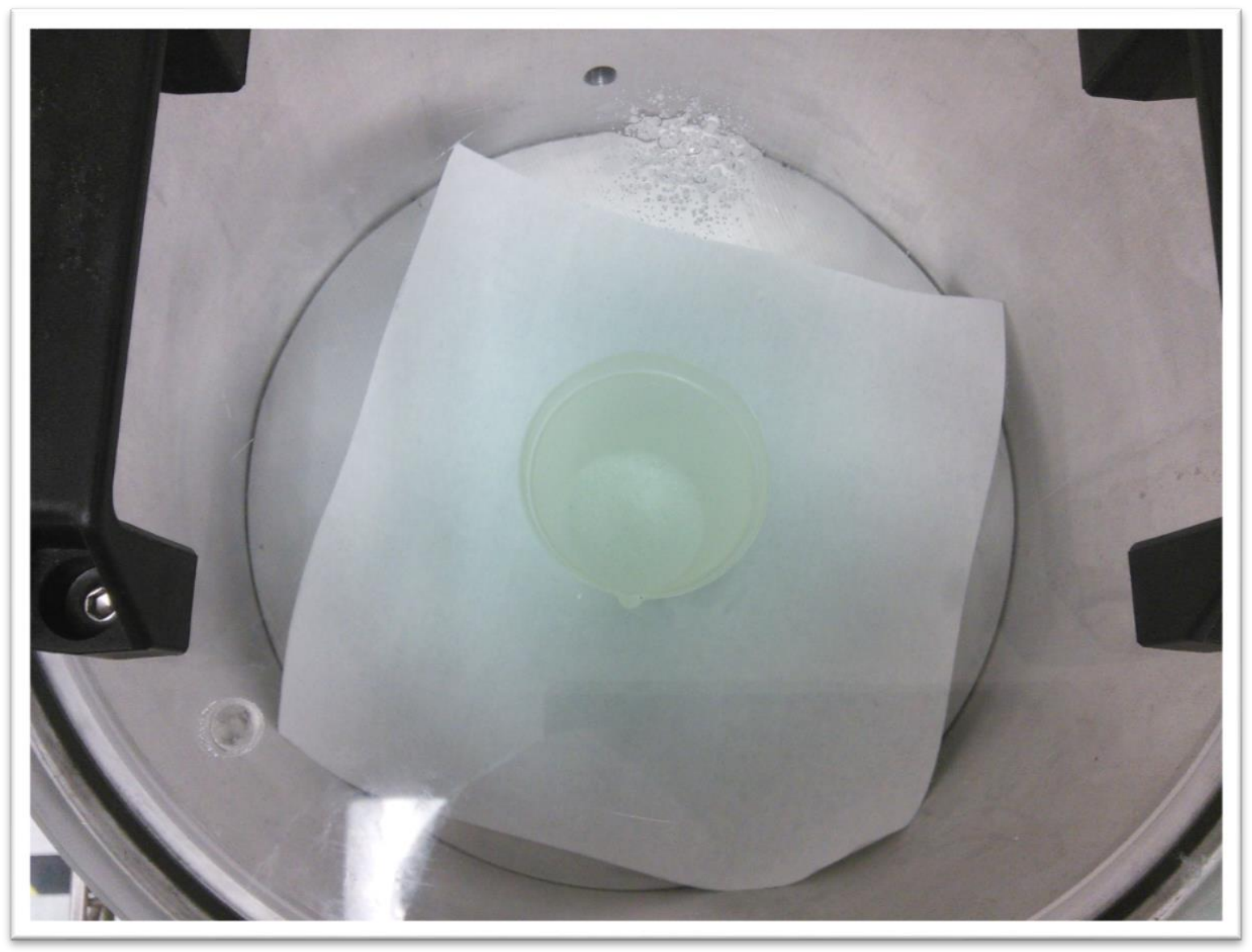

Figure 6.6 Chamber Vacuum Engaged with Rising Bubbles in Mixture Overall degassing generally takes anywhere from 10-20 minutes to remove all the bubbles from the mixture. Next the vacuum line valve was closed and the chamber was slowly vented back to atmospheric pressure. The degassed PDMS is removed from the chamber and ready for use.

\subsubsection{Mold 1}

The PET layer with the embedded circuitry is ideally placed onto mold 1 to obtain curvature. However, in practice, the PET layer with only the printed antenna had to be placed on the mold first and curved before 
placement of the following components: IC, display, and battery. To properly orient the antenna on the mold, the antenna was manually centered on the male component of the mold and taped into place on the side. The Female Mold 1 was then fitted on top of the Male Mold 1 and placed in an oven set at a temperature of 180 degrees Celsius. After 10 minutes, the mold was removed from the oven. Note that the oven and mold are both hot and it is important to insulate all body parts from the heat. Once removed, the mold was left to cool down to room temperature which took approximately 15 minutes. The mold was then separated resulting in the image below Figure 6.7.

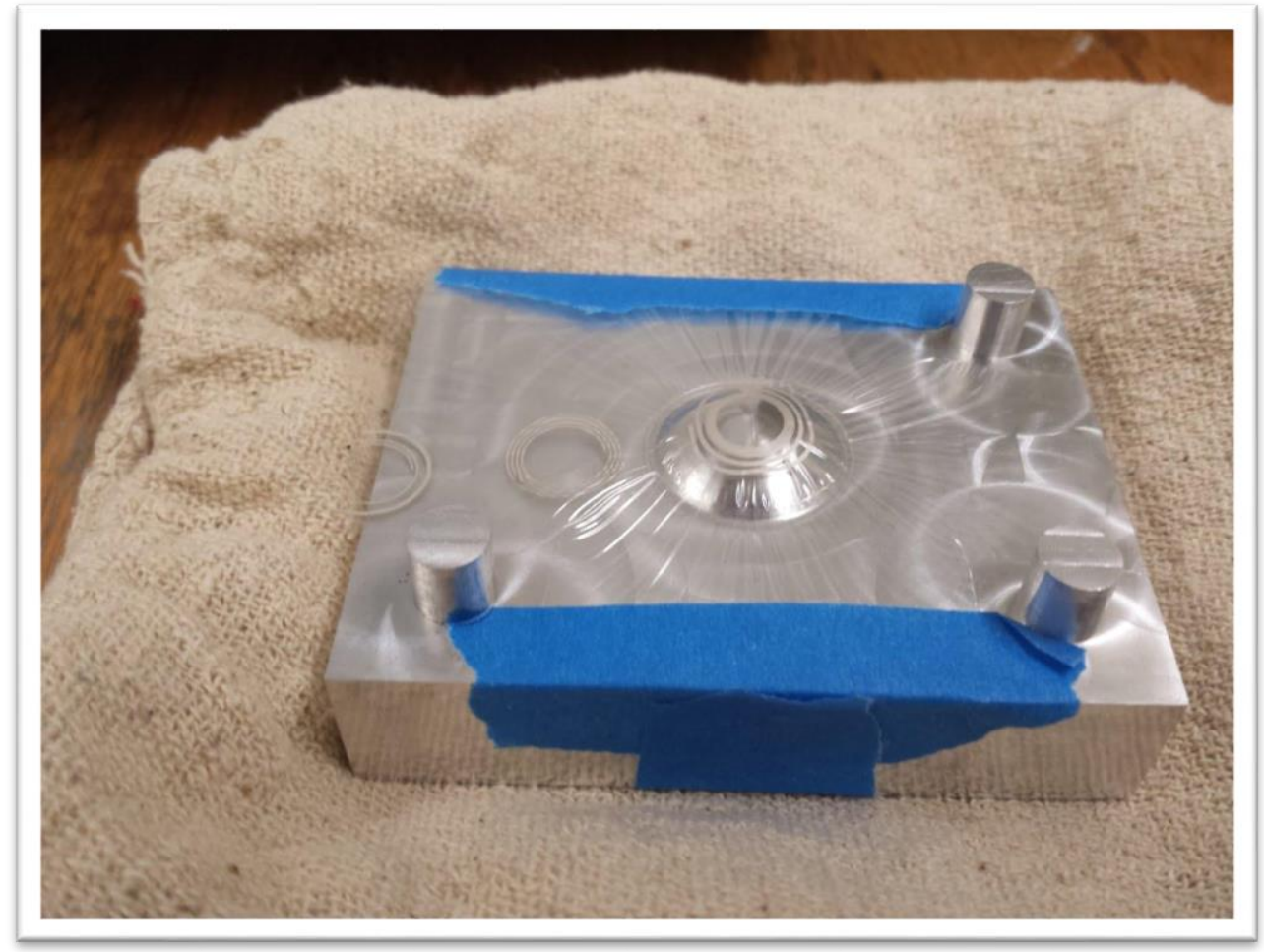

Figure 6.7 Molded PET antenna

The components were attempted to be attached to the antenna using a thermo-epoxy. This unfortunately failed because the tools were not small 
and precise enough to deal with pads that are on the micron scale. This would have to be completed in the near-future using a wire bonding machine where the processing steps for Electrical Engineering departments wire bonding machines are well documented in the next section of this chapter.

\subsubsection{Mold 2 and Mold 3}

The encapsulation of the lens in PDMS occurs in two stages with two separate molds. For the first mold, PDMS mixture was poured slowly into the bottom of Female Mold 2. The PET lens was then attached to the center of the male component, and placed component side down into the PDMS. A common issue with PDMS is the formation of bubbles during the pouring process. To overcome this, the mold was then placed in the degassing chamber to remove all bubbles that have formed during the pour. After, the mold was placed in the oven to cure for 4 hours at a temperature of 70 degrees Celsius. For the second stage of the process, the lens was placed PDMS side down into the third mold, and the PDMS was poured onto the PET side making a small pool which is shown below in Figure 6.8. The male component was then inserted, and the cavity formed creates a 50um layer of PDMS on top. The lens was then cured again for another 4 hours at 70 degrees Celsius. 


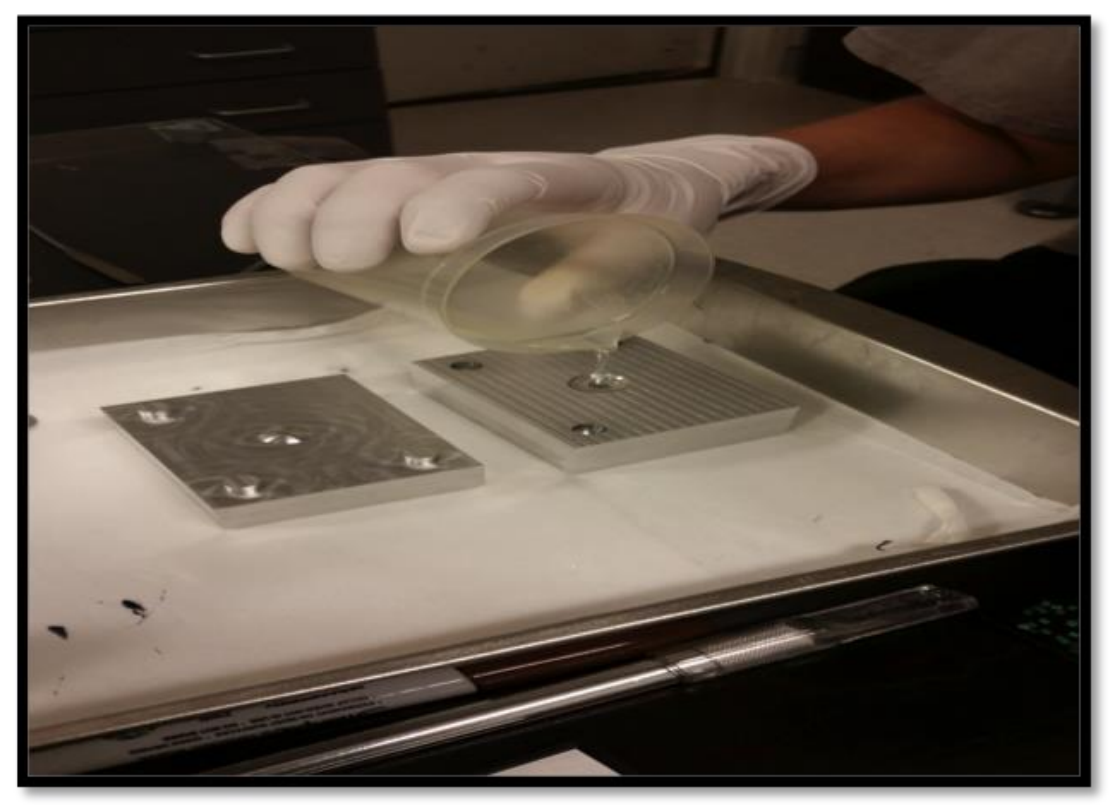

Figure 6.8 Pouring of PDMS to form external lens layer

Forming the PDMS encapsulation layer provided multiple challenges including separation of the mold following degassing and rough edges. Following curing of the mold, it was impossible to separate the male and female components by hand. Eventually, a razor blade and a hammer were used to pry it apart. It was found that excess PDMS had escaped from the center of the mold and dried in between the male and female mold faces. It was likely the bond between the PDMS and the mold was what made it difficult to separate the pieces. Notably this is not an uncommon occurrence in injection molding, and a simple solution would have been to machine a bevel in the side similar to make it easier to pry open. To remove the rough edges of the PDMS, manufacturing a separator similar to a cookie cutter would eliminate many of the problems associated with manual removal via $\mathrm{X}$-acto knife, such as rough edges and damage to the mold itself. 


\subsection{Wire bonding Machine}

The originally plan was to wire-bond the IC, battery, and antenna together in order to have a functional system. Dr. Smilkstein informed me that the EE and IME department on campus had wire-bonding machines and scheduled training with the company Small Precision Tools (SPT). Unfortunately there was not enough time to implement the wire-bounding due the tape out date for the second iteration IC which will arrive at the end of May or early June. Also the wire-bonding machines in the EE department are not operational and the trainer recommended to scrap the two machines and buy a working used one. This section documents the training I underwent to use as a reference for the next group that takes on this project. Figure 6.9 and 6.10 show 3 dimensional CAD drawings of the bonding configuration for the coil and IFA antenna. 


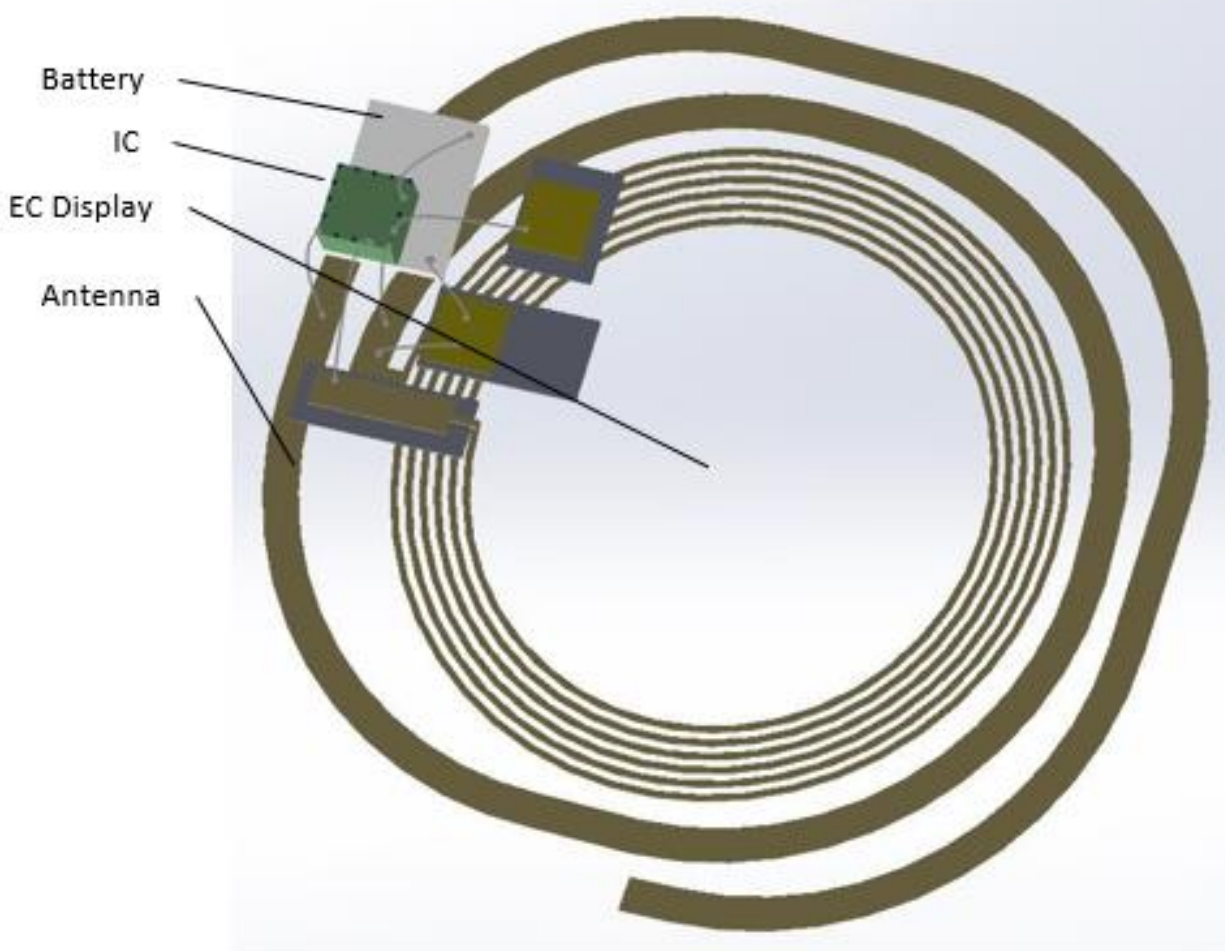

Figure 6.9 Bonding Configuration for IFA Antenna

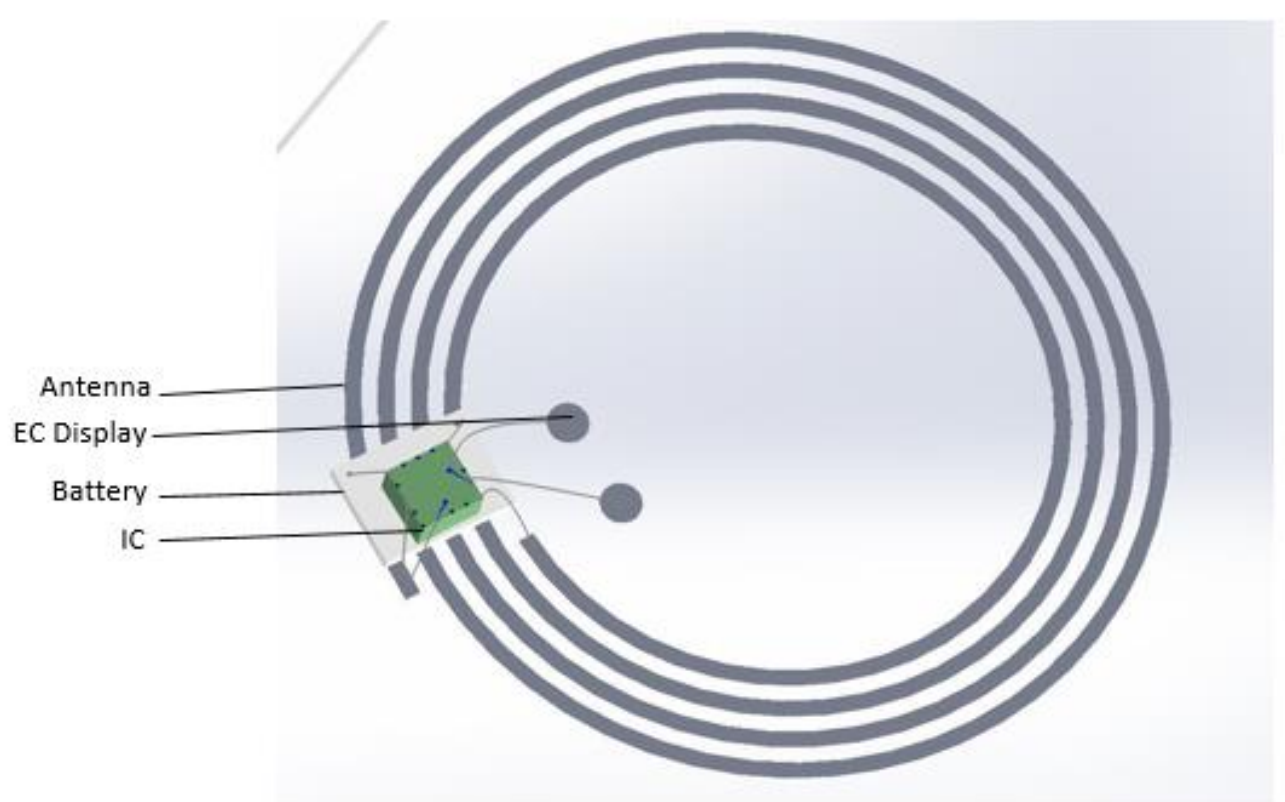

Figure 6.10 Bonding Configuration for Coil Antenna 


\subsubsection{Wirebonding Procedure}

There are two types of wire bonding, wedge bonding and ball bonding technology. The bonding machine I was trained on was a manually operated wedge bonder 5432 shown in Figure 6.11.

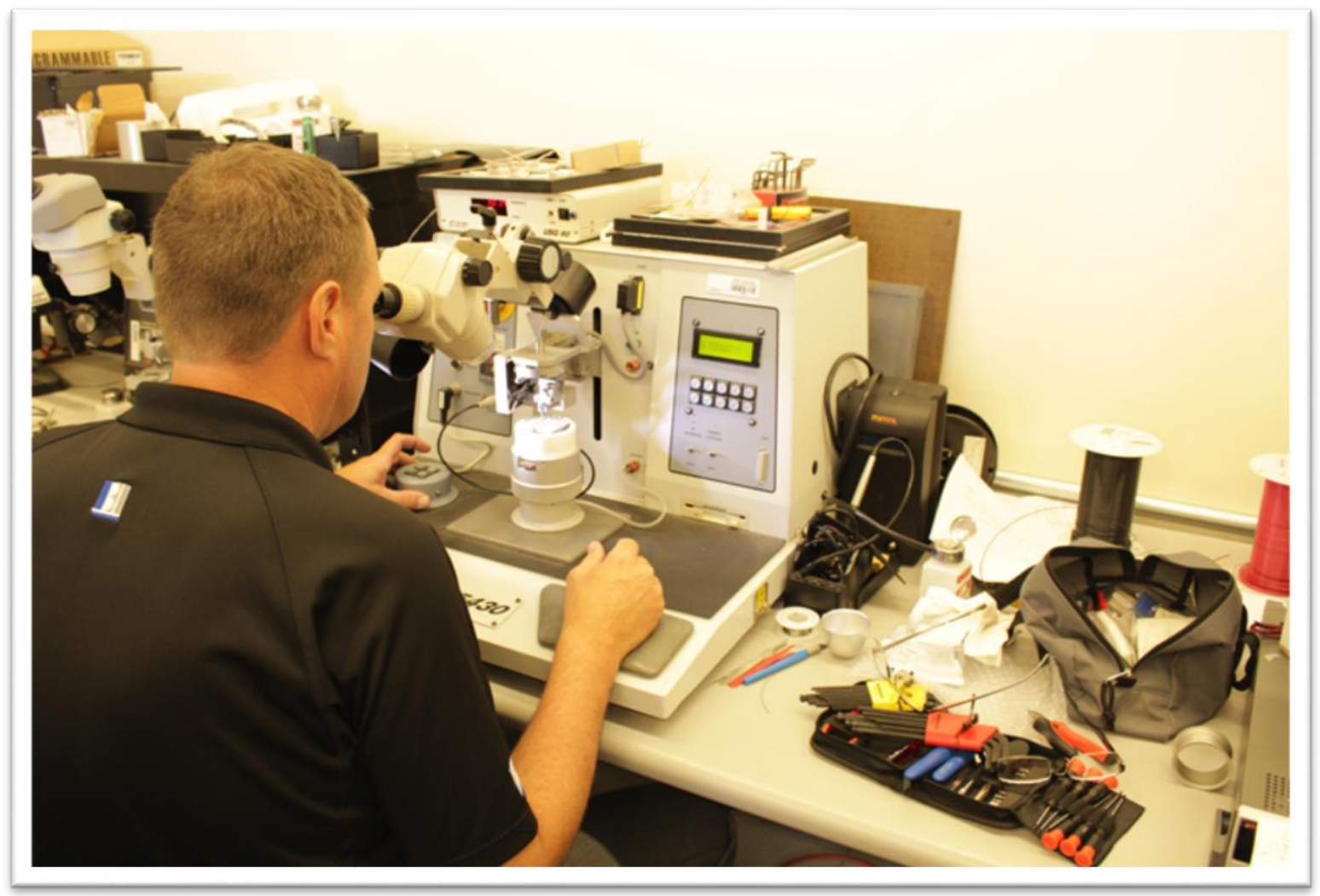

Figure 6.11 Manually Operated Wedge Bonder 5432 Machine

Wedge bonding technology is a process that uses ultrasonic to merge the wire to the pad by friction. The wire used in training was gold wire with a diameter of 25 microns ( $1 \mathrm{mil})$. In order to attach the gold wire to the pad, it needs heat at a temperature of $150^{\circ} \mathrm{C}$. The heat is needed because gold is a soft material and does not create the energy needed from friction to causes a reaction similar to diffusion between the pad and the wire. If aluminum wire was used, then no additional heat (room 
temperature) is needed to merge the materials. The wedge from the bonding tool must be 90 degrees perpendicular to the substrate's bonding pad. Figure 6.12 shows the configuration of the wedge bonder.

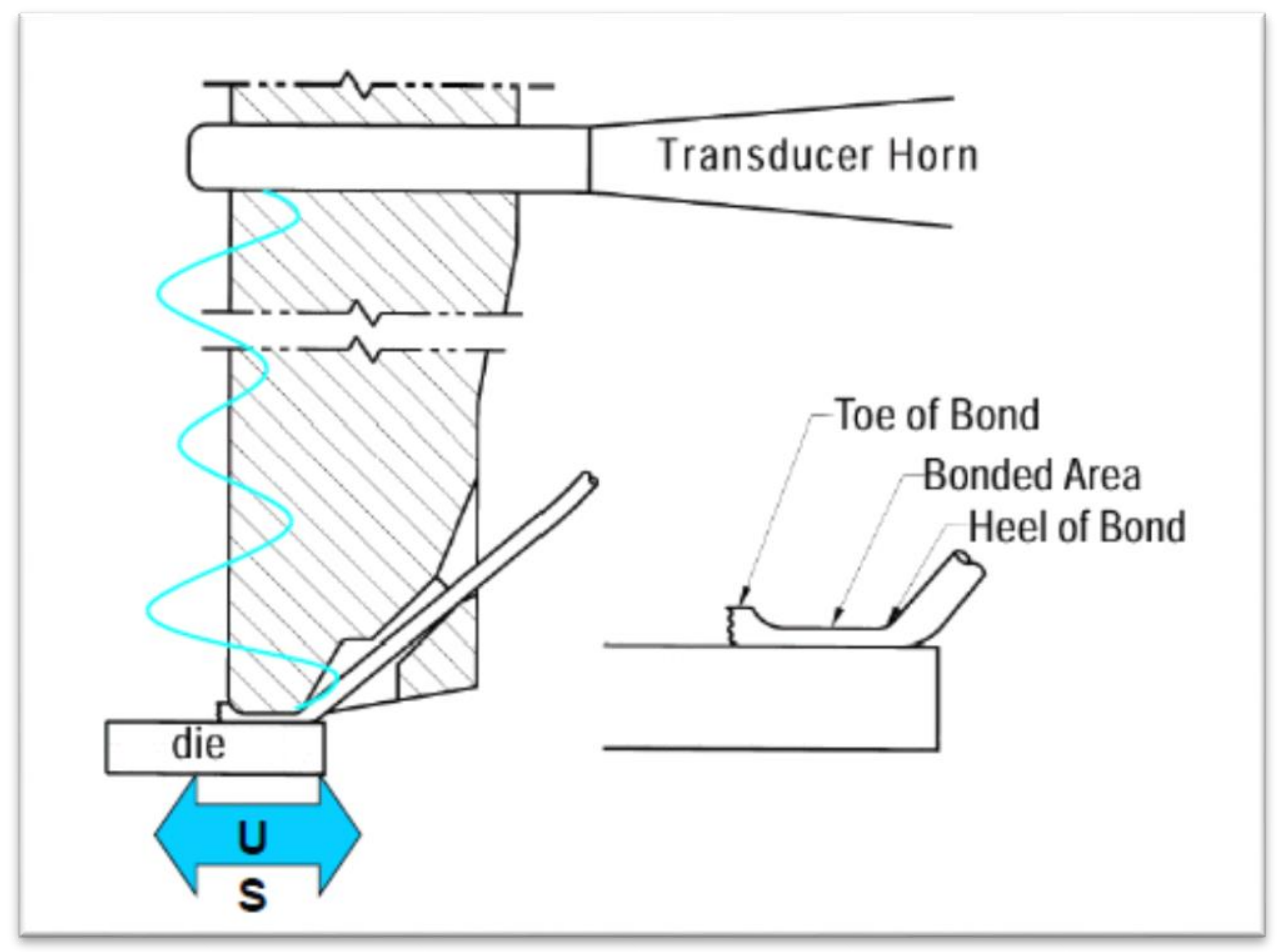

Figure 6.12 Diagram of Bonding Wire to Die Pad

The transducer horn on the top and the wedge tip is inserted. And the gold wire is ran through a small cavity where the wedge presses the wire against the die. Ultrasonic vibrations of $60 \mathrm{kHz}-100 \mathrm{kHz}$ are introduced to the wedge causing the friction needed for the bond. The vibration only last for roughly $20 \mathrm{~ms}$. In this process there are two bonds. The first bounds have a tail end wire where the length can be controlled. The second bond does not have a tail resulting in the bond. Figure 6.13 shows the results of the first and second bond. 


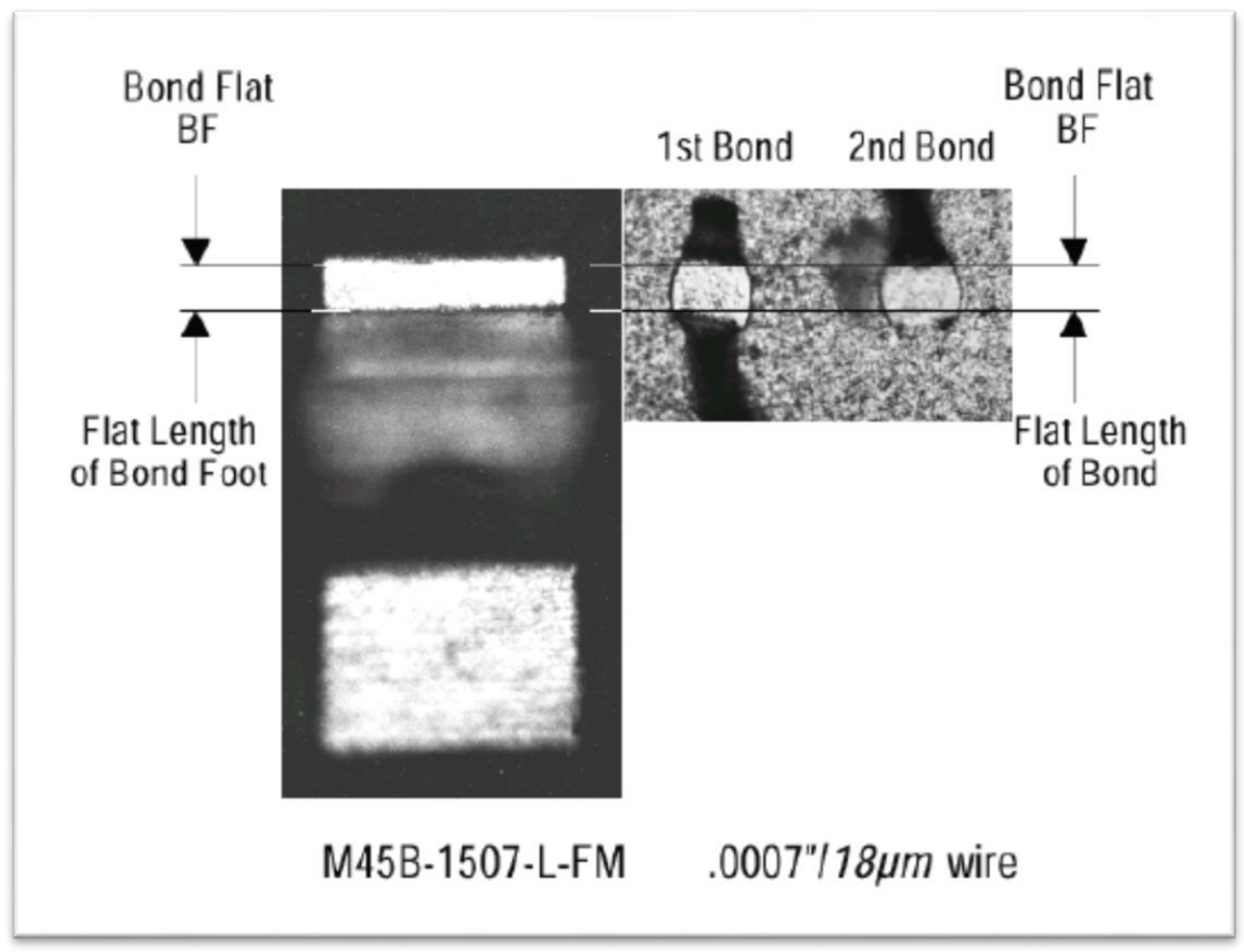

Figure 6.13 First and Second Bond

The machine allows the user to program the height and direction of the wire strand to manipulate the dimensions of the wire's loop. Figure 6.14 below shows the loop profile direction and the resulting shape of the wire. 


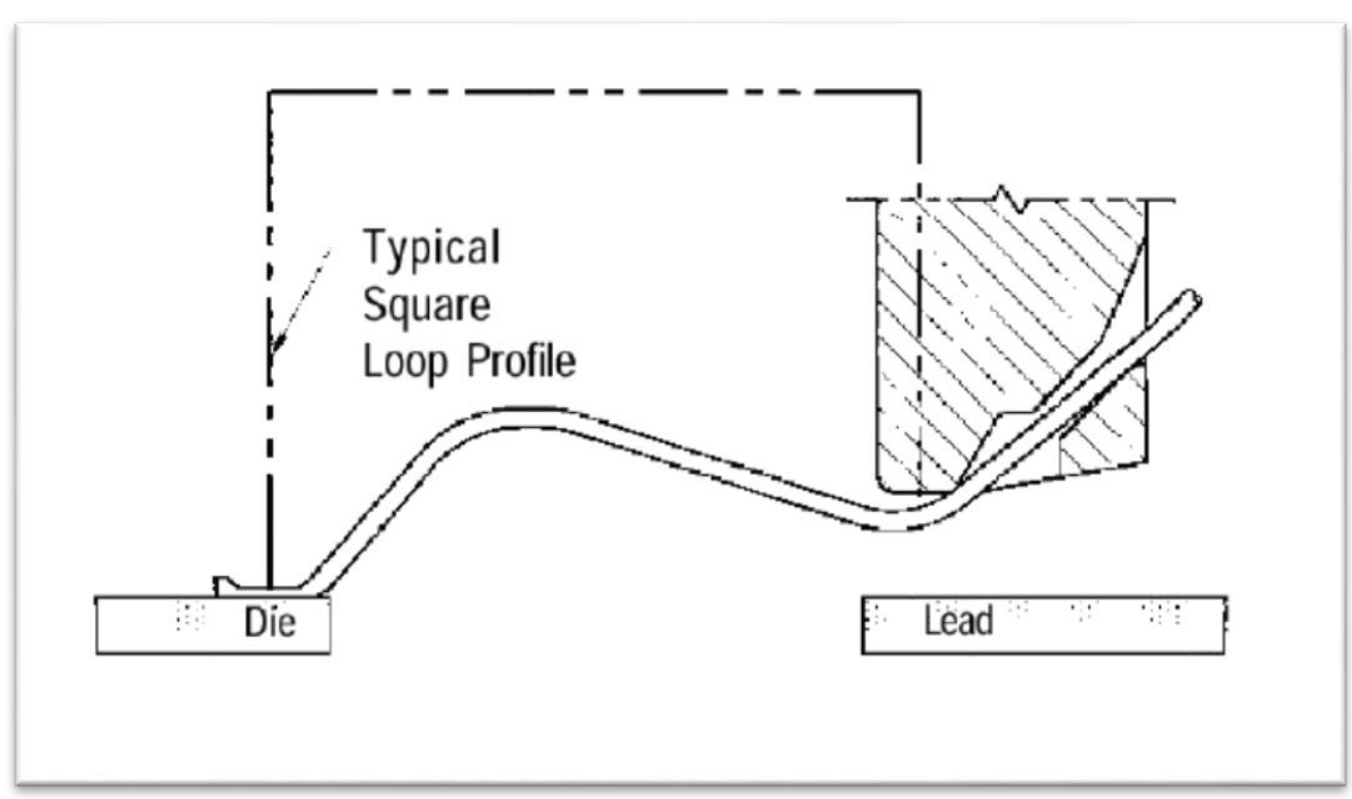

Figure 6.14 Loop Profile for Shaping Wire

To make bonding successful the proper wedge and wire is needed. For the wedge the diameter of the cavity must be $2-3$ times wider than the diameter of the wire. Generally the diameter is 2 times the diameter of the wire because if the cavity is too big, generating the loop can be difficult. The wire feeding angle of the cavity is usually 45 degrees, however, depending on the application another angle might be better. The final step is the process of bonding is termination. Termination is when the wire clamp closes after the second bond the wedge will lift and the wire will break in the area where the tip of the wedge closest to the cavity was located during bonding. Figure 6.15 shows an illustration of the termination process. 


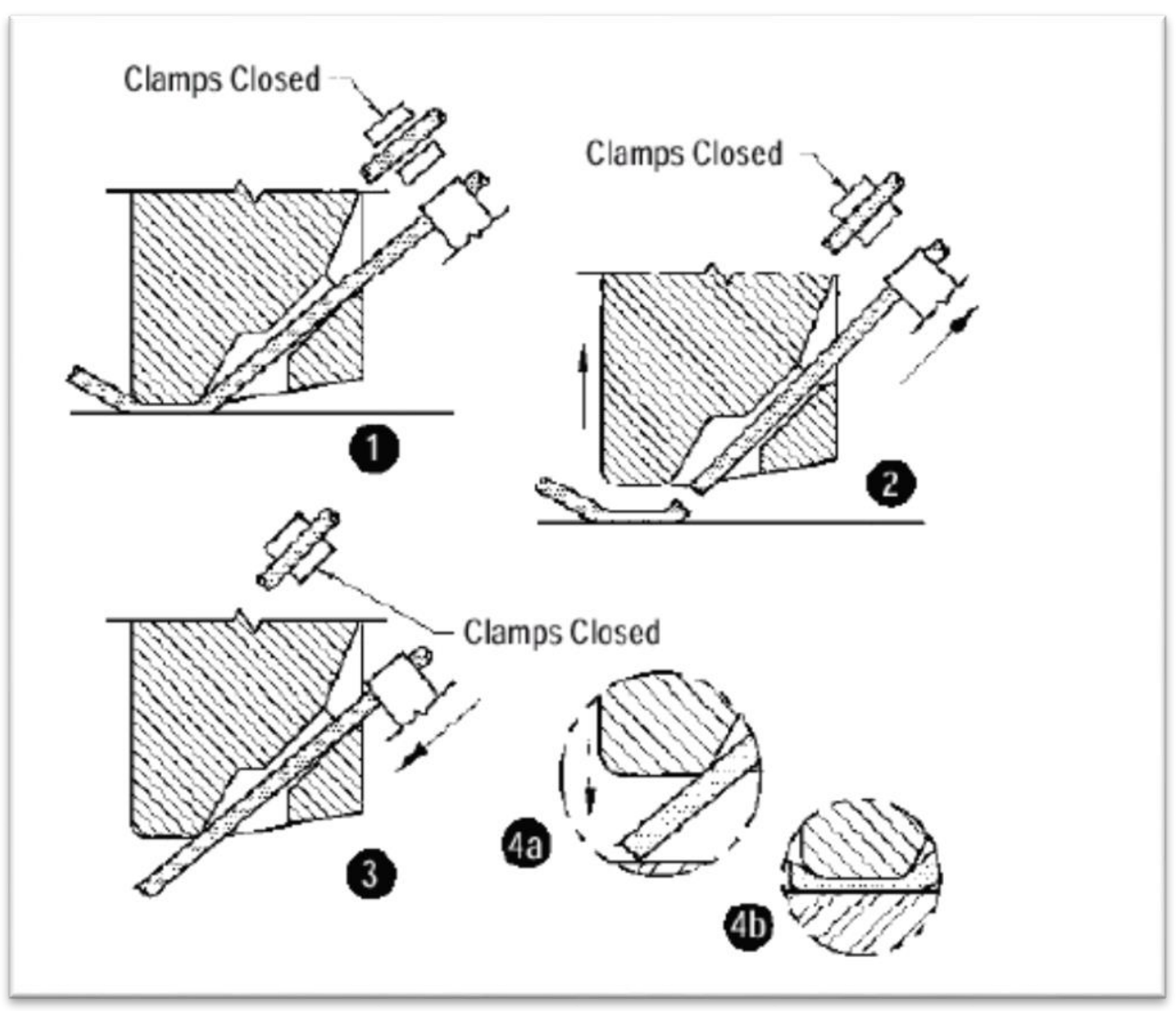

Figure 6.15 Termination Process

In order to calibrate the machine a sheet of gold pads called coupons are used. This served as a reference to set the machine to its default settings and to ensure that the machine is functioning properly. Figure 6.16 below shows the coupon being used for calibration. 


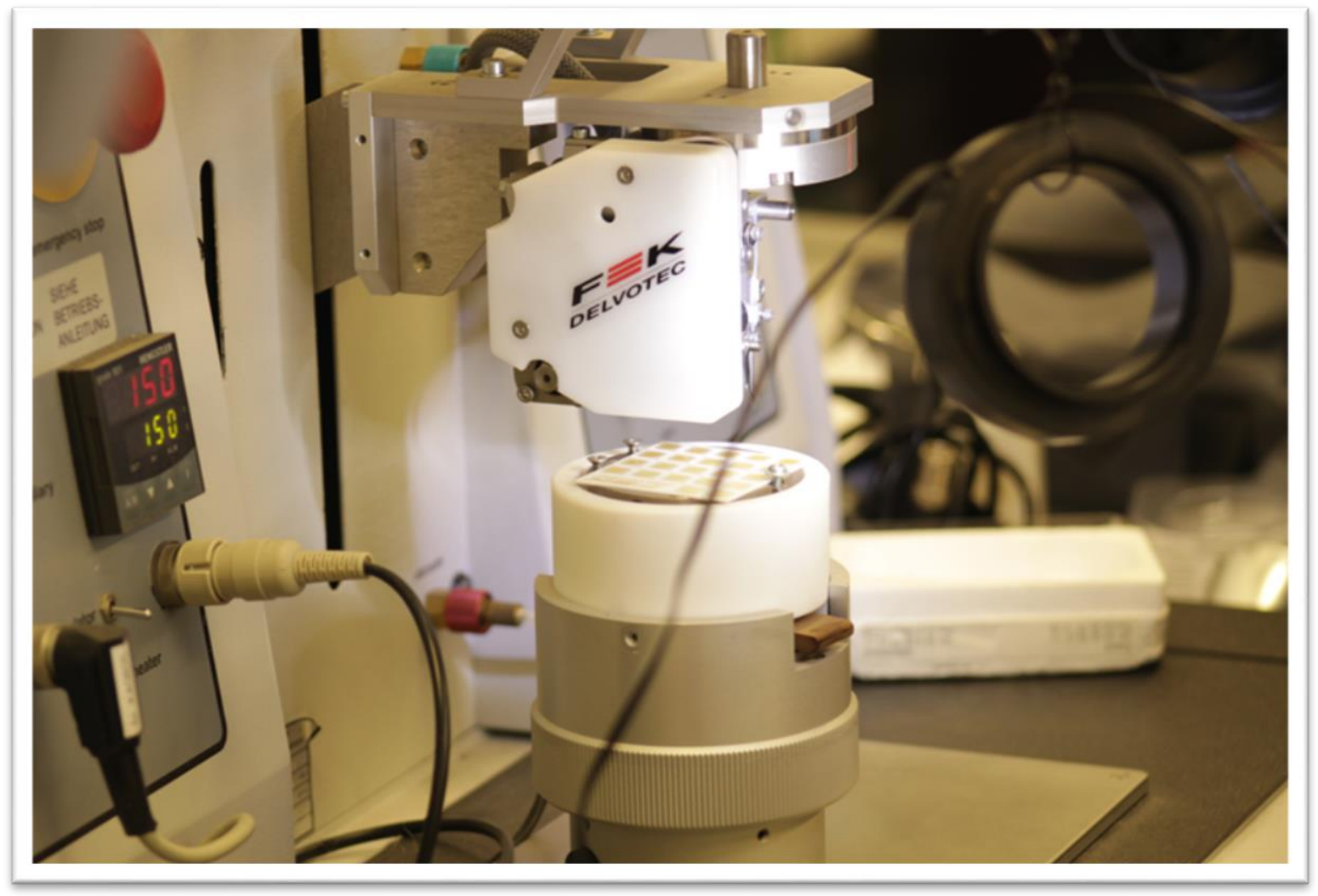

Figure 6.16 Coupon Used for Calibration

\subsection{Material Properties}

\subsubsection{Biocompatibility}

The purpose of biocompatibility assessment was to determine whether the final design improved upon previous designs which exposed PET to the surface of the eye. PET, as stated in our preliminary analysis, has been shown to yield endocrine disruptive properties. Additionally, PET is traditionally characterized a "hard" contact lens material, with a high elastic modulus and characteristically rough surface. This makes PET fairly uncomfortable when placed on the human eye for long periods of time. In our final design, we purposely encapsulated PET with PDMS to 
circumvent some of the uncomfortable aspects of PET with a softer, more flexible polymer.

Materials selection should be noted here as a point of special consideration. PET was found to be, according to a study conducted in 2010, an endocrine disruptor when exposed to a biological system [17]. This is a definite problem when applied to all three designs. A design that incorporates a barrier between the PET and the surface of the eye would be the most desirable to counteract this finding.

While literature reviews of PDMS have indicated it is a biocompatible material with minimal side effects we do not advise usage of lens for extended periods of time. Both in vitro and in vivo testing must be done before the lens can be safely used. These tests are also critical, because they account for parameters that are difficult to measure, such as the leaching behavior of the PET, electrochromic ink, battery, or substances from the bare die IC using simulation. At this point, safety concerns have been addressed as much as possible to ensure a device that is both safe and effective.

A staple of biomedical design is the process of determining the suitability of a material in a medical device application. This means that the material must not do harm to the body within the intended function, and the body must not do harm to the material. This is by far the most difficult requirement to contend with from an engineering perspective because it does not have a quantifiable nature. Instead, biocompatibility analysis is 
performed during acute and chronic studies on cells, living systems, and even live patients. The data from these studies is then compiled and analyzed looking at notable examples of bioactivity such as inflammation, systemic toxicity, and rejection. Since the product will be in direct contact with the user's eye for the duration of their task, any material selected as the base for the lens must not be sufficiently biocompatible.

Many commercial polymers used in the biomedical industry have already been characterized for biocompatibility. This, however, can be a double edged sword. This data may not be for the region of interest, i.e. the surface of the eye. Also, this data can limit the materials selection process significantly, to the point where a suitable material may not currently exist. As such, determining a material from the perspective of biocompatibility is a peripheral effort: it is impossible perform studies with resources currently available. A reliance on currently available data will be the primary means of analysis of biocompatibility. Currently, both PET and PDMS are characterized as relatively biocompatible, with the former being a known irritant due to its relatively low oxygen permeability when compared to PDMS. PDMS stands currently as the best material for biocompatibility when in contact with the eye, as many modern contact lenses utilize PDMS as a substrate material. This means the usage of a PDMS substrate would significantly strengthen the pursuit of a $510(\mathrm{k})$ clearance with the FDA. 


\subsubsection{Clarity}

The clearness of the lens is largely governed by the material used to fabricate the lens, as well as any surface coating applied to the lens surface. The refractive index of a material, $\mathrm{nD}$, is a dimensionless number that describes how light and other radiation propagates through a material. It is obtained by the following equation, which divides the speed of light in a vacuum by the speed of light in a substance:

$$
n=\frac{c}{v}
$$

Where $\mathrm{c}$ is the speed of light and $\mathrm{v}$ is the velocity of light in the medium. Since the prototype will not be a prescription lens, the best fit material would have a refractive index close to 1 , meaning that light entering through the lens would be refracted a very small amount, and the individuals vision would not be blurred significantly. This, along with biocompatibility and flexibility, governed the materials selection process. It is important to note that achieving a refractive index of 1 with commercial polymers was unlikely. Commercially available polymers have refractive indices that range between 1.3 and 1.7 [18]. As such, we have determined that a refractive index between 1 and 1.5 would be acceptable for our application. This range of indices was acceptable because the eye itself will refract light based on a desired level of acuity. If the refractive index is within the range specified, the eye should be able to distinguish objects without any loss of acuity. 
Also the placement of any circuitry on the lens was taken into accountability. With the current design, the pupil area of the lens must be free of any and all obstruction by circuitry. This includes up to $9 \mathrm{~mm}$ in diameter of obstruction-free material. Any circuitry on the lens must be clear circuitry or be such that the eye will not be able to resolve the obstructing items at any time.

The focus of testing was to assess the clear vision characteristics of the material and ensure that its acquired data was close to the theoretical amount to satisfy the acceptable bounds. This will ensure the resulting system would cause the least interference with the users vision at all times. As such, elements such as refractive index, circuit geometry, and contact angle were analyzed.

\subsubsection{Refractive Index Testing}

For refractive index testing, a simple fixture was created using the following equipment found in the Biomedical Engineering Lab of Engineering IV.

- $650 \mathrm{~nm}$ wavelength $10 \mathrm{~mW}$ laser pointer

- Solder assist clamps

- Tape measurer

- Leveler

- Protractor

- White board and whiteboard markers

Figure 6.17 on the next page shows some of the materials used in proving the fixture. 


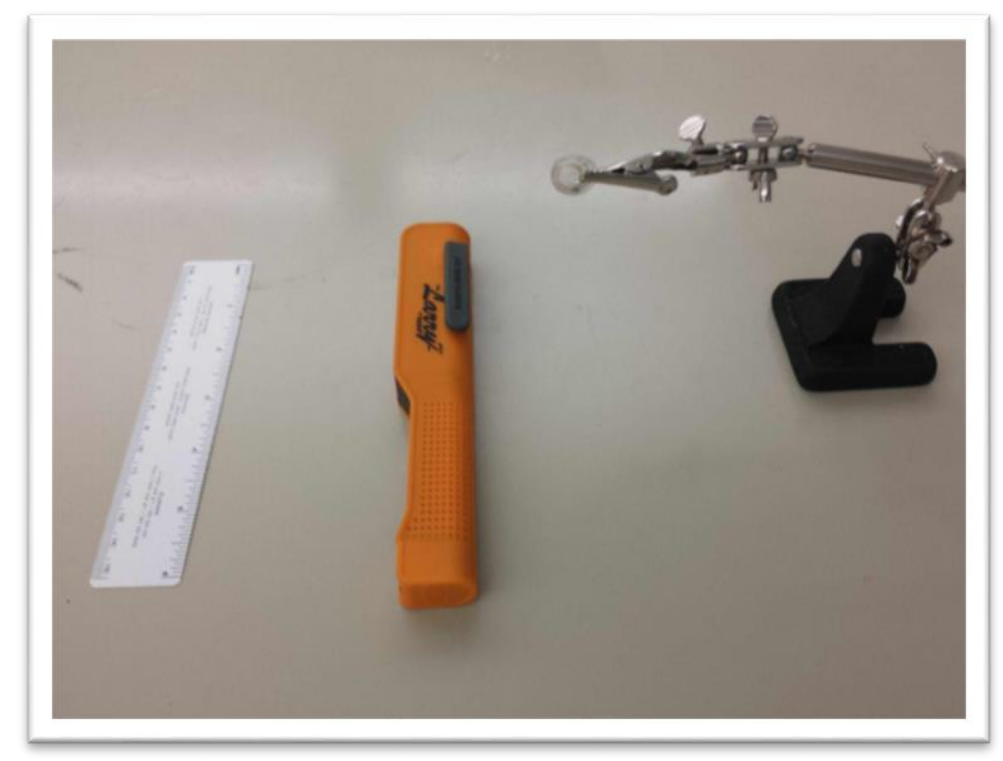

Figure 6.17 Materials used in designing refractive index test fixture The fixture involved attaching the laser pointer to a protractor, placing it on a level surface, and fixing the test subject at a point at least 2 feet from the laser pointer. The laser would then be directed using the protractor at the subject material, in this case the contact lens, at varying angles. A white board placed behind the lens would be used to mark the location of refraction through the lens, and after measuring the $\mathrm{x}$ and $\mathrm{y}$ distances from the lens, the angle of refraction would be computed using trigonometry. Using Snell's law, the refractive index of the lens would then be determined. After 15 different attempts, data collected was discarded due to a standard error that exceeded a tolerance of more than 3 standard deviations away from the average.

This test ended inconclusively for a number of reasons. The first most jarring reason was the difficulty in controlling the absorption of the laser as it entered the lens. Since the lens is a compound material composed 
of two PDMS layers around a PET skeleton, there is a high chance that some of the light will be absorbed and dispersed as it passes through the lens. This effect was so noticeable, in fact, that the prototype incurred thermal damage when examined immediately following the first test. To account for this effect, future tests should include calculations to determine if the effect of absorption will damage the lens, and therefore make determining refractive index impossible. Another challenge associated with ascertaining the refractive index is the nature of the test itself. At the time when the tests were performed, it was assumed that the light exiting the lens would be refracted. This is, however, not correct, as light exiting the lens will retain the angle at which it entered, since it is reentering the medium in which it originated. This is in accordance with Snell's law of refraction.

$$
\frac{\sin \theta_{1}}{\sin \theta_{2}}=\frac{v_{1}}{v_{2}}=\frac{n_{2}}{n_{1}}
$$

As such, it may be more appropriate to purchase a refractive index tester, such as one manufactured by Presidium Electronic, to determine the refractive index accurately. One other solution would be to find manufacturing method to make the entire lens on one type of substrate. If the lens was complete made of PDMS the refractive index would be 1.4.

\subsubsection{Flexibility}

The next requirement that largely impacted the design was flexibility. Any 
material chosen to support the design must be able to flex to the curvature of the eye surface. It must also resist significant stress from curving to avoid damage on the lens itself. The method of quantifying flexibility relies on the computation of the stiffness, $k$ of the material. This is given in relationship to the young's modulus $E$ by the following equation:

$$
k=\frac{A E}{L}
$$

Where $A$ is the cross sectional area of the sample and $L$ is the length of the sample. In general, the higher the value of $k$, the stiffer the material becomes. In materials selection, this means that the higher the young's modulus of the material, the stiffer it becomes per element. However, the lower $\mathrm{k}$ is, i.e. the more flexible the material is per element, and the lower the young's modulus becomes. A material with too low a young's modulus can be at a higher risk of plastic deformation, and in the worst case, failure. Failure in this category is defined by exceeding the ultimate tensile strength of the material and resulting fast fracture. It is important to note that the above equation represents axial stiffness. In the current problem, generalizations regarding the bending of the lens as a two dimensional stiffness problem can be made by assuming young's modulus is isotropic for the contact lens.

The target for flexibility is measured directly by Young's modulus, E, of the material chosen. A large range of these values are listed in Appendix A. This variance is a result of the two top material candidates having 
opposite flexibility, with PDMS being relatively compliable compared to PET. Another factor which is directly impacted by flexibility is the integrity of any onboard circuitry. The circuitry must be able to bend without incurring significant damage or wear.

\subsubsection{Comfort}

Contact angle testing was used to primarily determine the wettability of the surface of PDMS, the exterior component of the final lens design. The materials used for this test included a sample of PDMS encased PET a VCA Optima contact angle system, saline, and DI water. Figure 6.18 below depicts this test in progress.

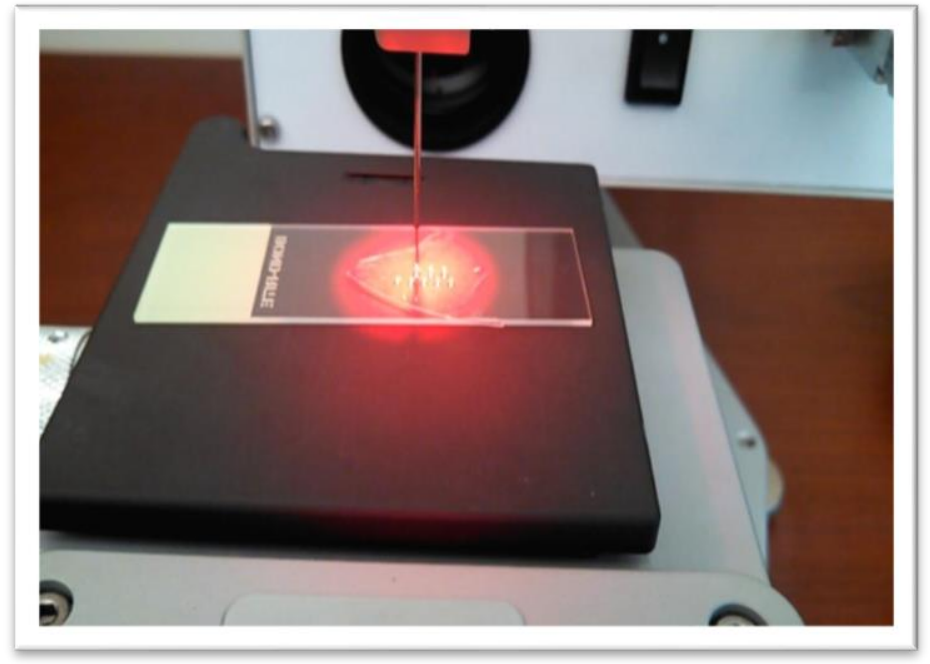

Figure 6.18 Contact angle test in progress using VCA Optima system

The results of these tests were very clear: PDMS is Hydrophobic and not nearly wettable enough to provide a comfortable, clear adhesion of the prototype lens to the human eye. Contact lenses rely on the surface tension created by the tear layer between the eye and the lens to remain in place. If a material with insufficient wettability is placed on the eye, the 
tear layer will glob up and areas of the lens will be in direct contact with the eye, causing irritation and blurry vision. Results from this test were computed and are presented in figure 6.19 below.

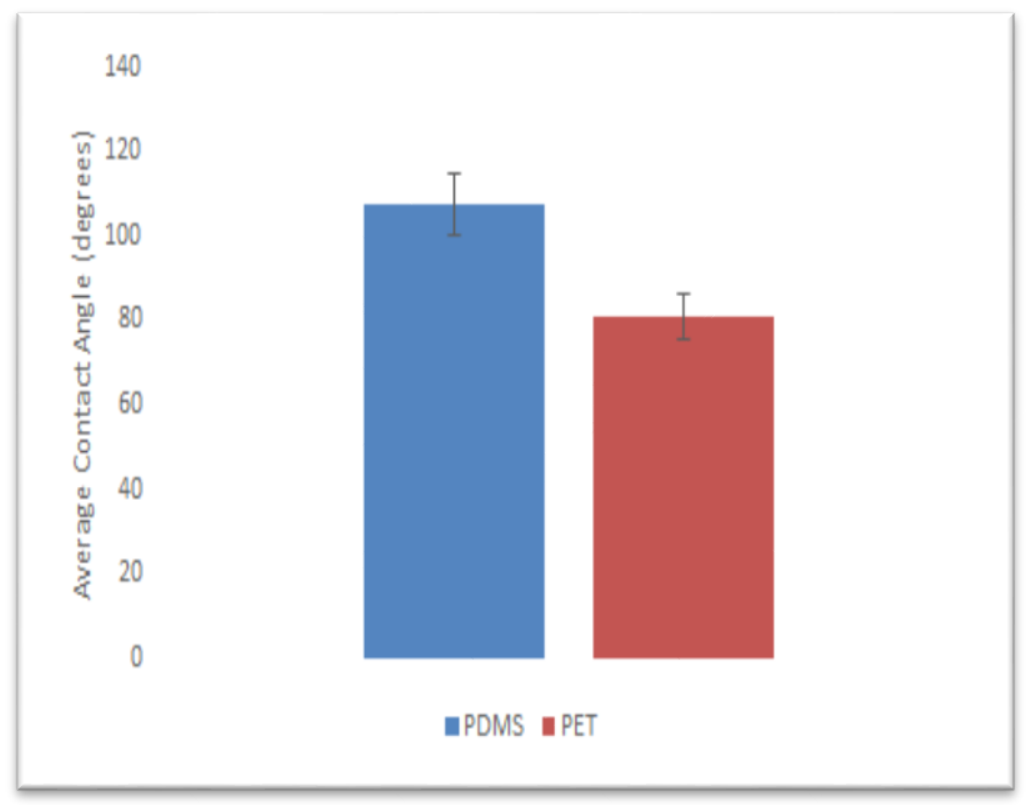

Figure 6.19 Bar chart of average contact angles for PET and PDMS, including standard error bars. $(n=15)$

It can be shown in the figure above, PDMS has a contact angle of around $105^{\circ}$, far exceeding the upper limit of $65^{\circ}$ set in the final specification. The most straightforward way to remedy this large contact angle would be the use of a surface hydrogel coating for contact lens. Silicone hydrogels are contemporary materials developed in 1999 to solve the problem of contact lens induced ocular hypoxia. They are highly permeable, and have a desirable contact angle for use in contact lens applications. Traditionally, they are cut using a lathe from a solid block of material. This would make applying to the surface of PET difficult. Recently, however, a patent filed under the number WO2009073401 A2 proposes the creation 
of a novel silicon hydrogel spray for use in tissue dressing application [19]. Should this technology come to fruition, it would be an excellent substitute for PDMS as an exterior lens material. A third option would be to use a high experimental polymer from momentum which google is currently using on their version of the lens. It is biocompatible and has a contact angle of lower than $60^{\circ}$ which means that it would have the most amount of comfort because the material property is more hydrophilic. I was able to negotiate with momentum and they have agreed to allow Cal Poly to use this polymer for our lens research. This will hopefully help the next group encapsulate the circuitry with no problems of adhesion and wrinkles during encapsulation. Also this will open up a new variety of processing on the printing side since the surface energy of the polymer is relatively high compared to PDMS which made it nearly impossible to print on.

\subsection{Power Dissipation and Bio-heat Transfer}

Modern electronics run largely on AC dynamics, and as such, power generated and used by a circuit will consist of two components: real power and imaginary power. From a systems perspective, the power that has the potential to be dissipated as heat comes in the form of real power, given by the following equation:

$$
P=\frac{1}{2} V_{p} I_{p} \cos \theta=V_{r m s} I_{r m s} \cos \theta
$$

Where $\mathrm{V}_{\text {rms }}$ represent root mean square voltage, Irms represents root 
mean square current, and $\theta$ represents the phase angle. This power is in units of Watts, and is consumed by the system in question. Calculations can be done after obtaining real power to determine the heat dissipated within a specific timeframe of use, by multiplying the real power by the total active time. In this design, the active portion of the contact lens must generate and use a little power as possible, due to the danger of excess heating in the eye and electrical safety. Comfortability of the user will directly depend on how hot the circuitry becomes.

It was assumed that the system will not become hot enough to damage the onboard circuitry (i.e. temperatures above 60 degrees Celsius.) COMSOL simulations performed indicated that given the maximum power output of $10 \mathrm{~mW}$. In this regard, maximum allowed change in temperature is given as 1.5 degrees Celsius, and was tested with PET, PDMS, and combination lens models.

\subsubsection{COMSOL Simulation}

The goal of simulations performed was to characterize the thermal effects of the active contact lens on the human eye for prolonged periods of time. Simulations were performed with both candidate substrates, PET and PDMS, and circuitry was simulated as a constant power source. Bioheat transfer analysis based upon the Pennes bioheat equation was performed in COMSOL with a close approximation of the finite element model proposed by Gokul K.C. et al. [20] 


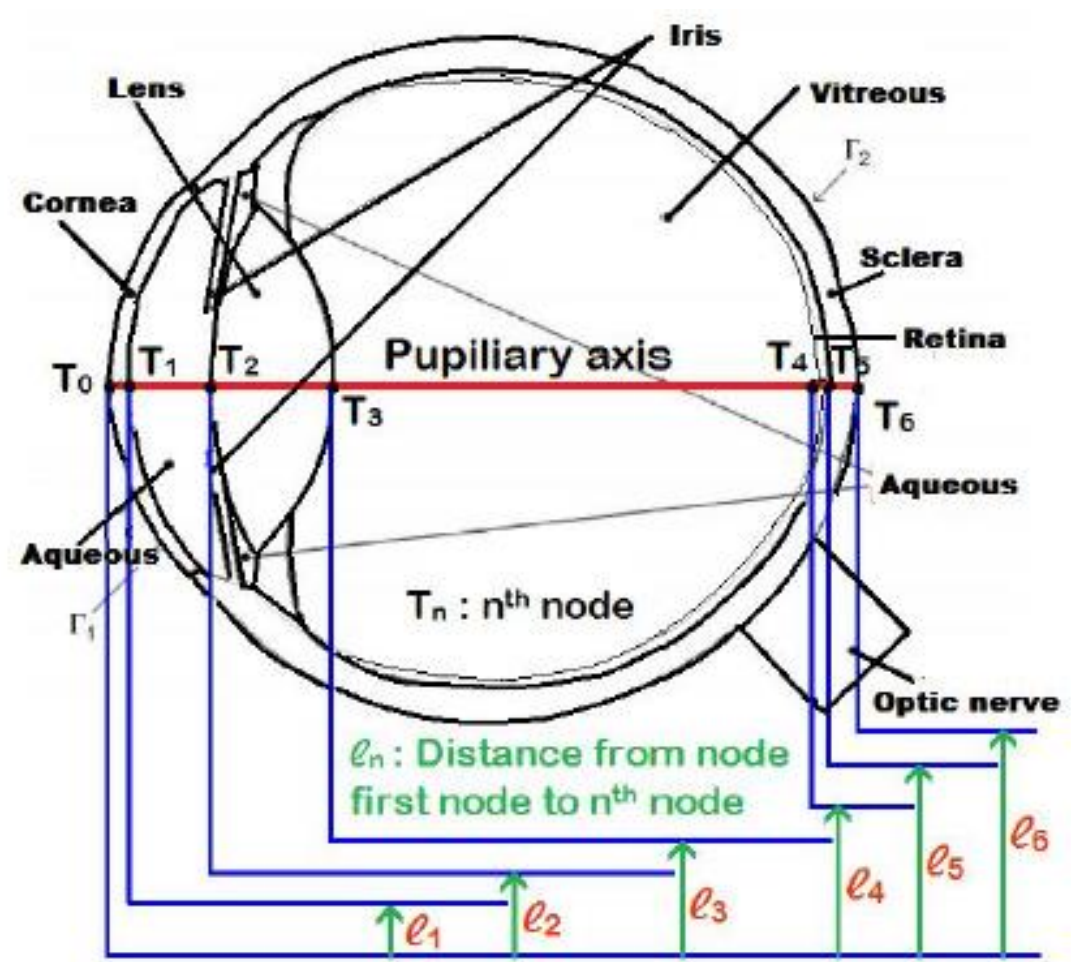

Figure 6.20 Proposed Finite Element Sketch of Human Eye

Where T0 through T6 represent nodal temperatures up to the $n$th element of the model, and L1 through L6 represent distances from the surface of the cornea to the $n$th element of the model. The above model was used primarily as a reference in which to approximate our simplified model, seen below: 


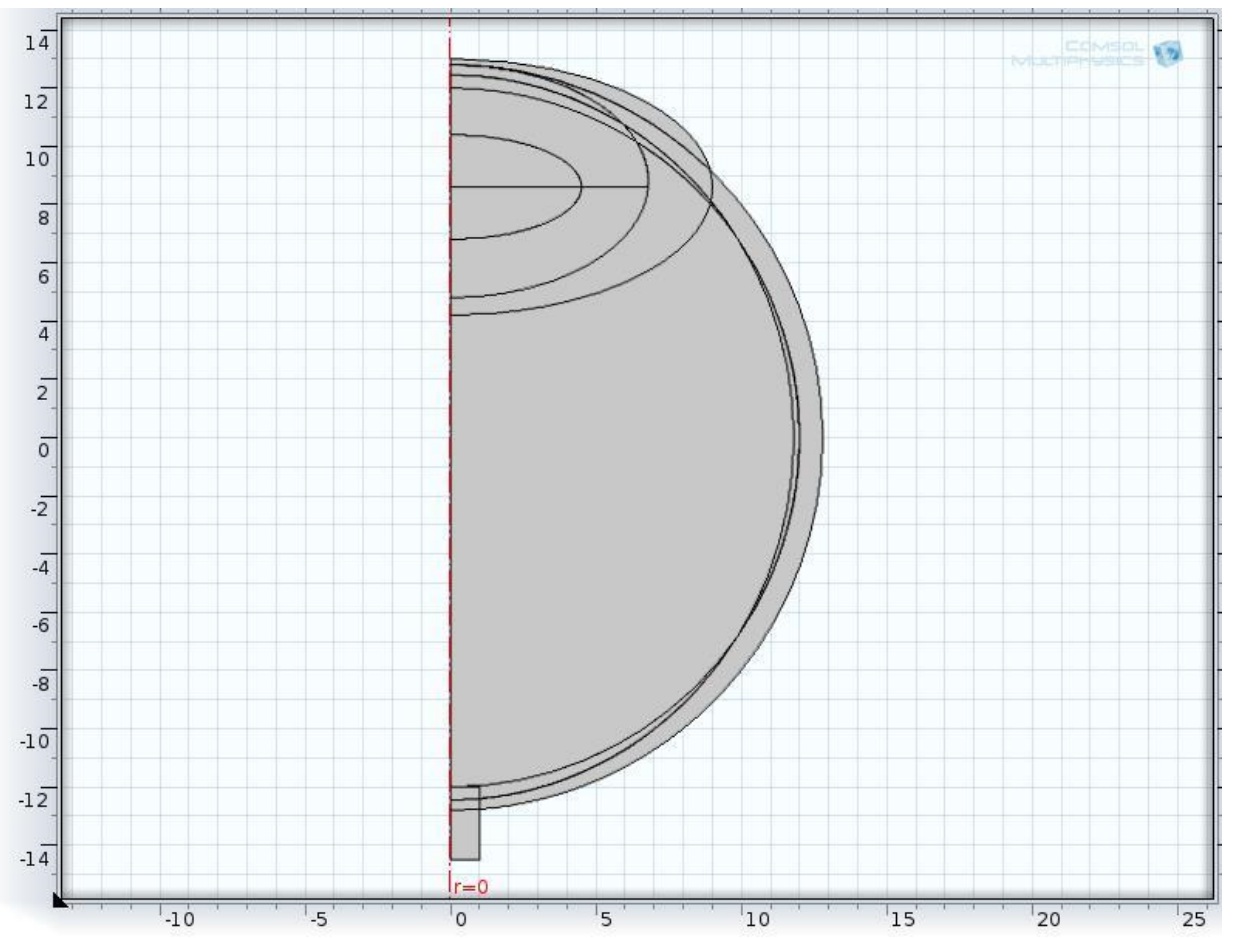

Figure 6.21 Axisymmetric COMSOL Sketch of the Human Eye

The above model was simplified significantly primarily to reduce computational time required in COMSOL and to reduce complexity of the resulting temperature distribution. Also, it can be seen that at the top of the model, around $\mathrm{r}=12 \mathrm{~mm}$, we have included an approximation of the contact lens, with a varying thickness of 250 to 400 micrometers. The thickness is such that at the center, it is smallest, and at the edges, it is largest. This variance was designed in order to remove the need for a complex Bezier polygon geometry when simulating the contact lens in COMSOL.

In this model, we assumed that the temperature of the surface of the cornea was 35 degrees Celsius, 2 degrees Celsius lower than the rest of the eye. There are two primary reasons for this assumption. First, convective heat flux at the surface of the eye associated with tear flow 
and gas flow naturally reduce the surface temperature of the cornea.

Second, surface to ambient radiation will further reduce the temperature of the surface of the eye as temperature increases. Blood perfusion rates within the ciliary body and retina and various thermal properties of the eye were extracted from works by Gokul K.C. et al. and Cvetkovic et al. [21]

Using a $10 \mathrm{~mW}$ total power dissipated by the contact lens, the following temperature distributions were obtained from a time dependent simulation with 180 second end time and 0.1 second step size for both PET and PDMS:

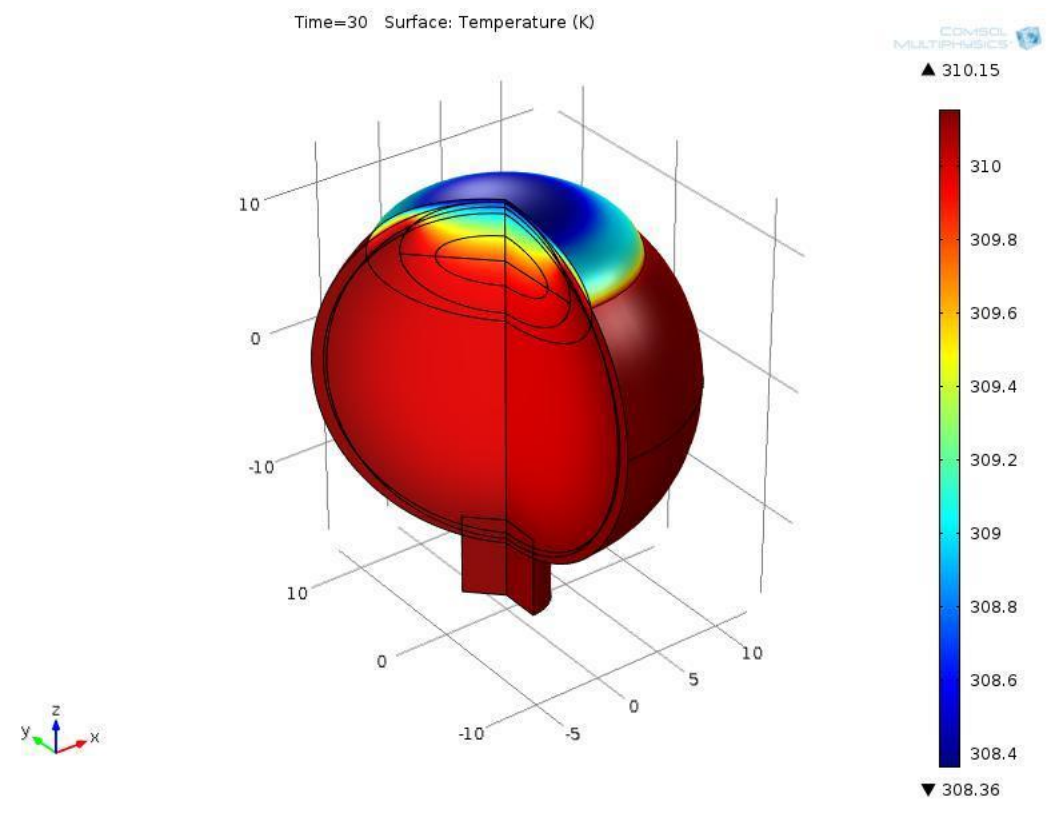

Figure 6.22 3D Surface Temperature Distribution after 30 Second ExposurePDMS 


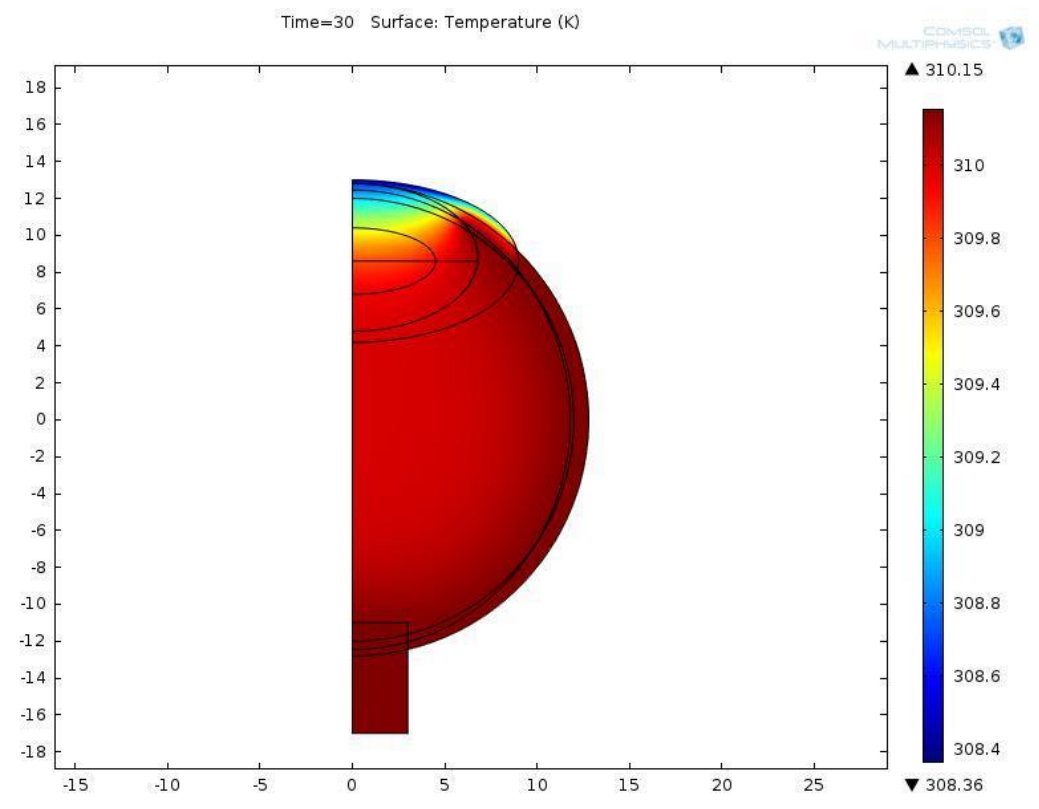

Figure 6.23 2D Surface Temperature Distribution after 30 Second ExposurePDMS

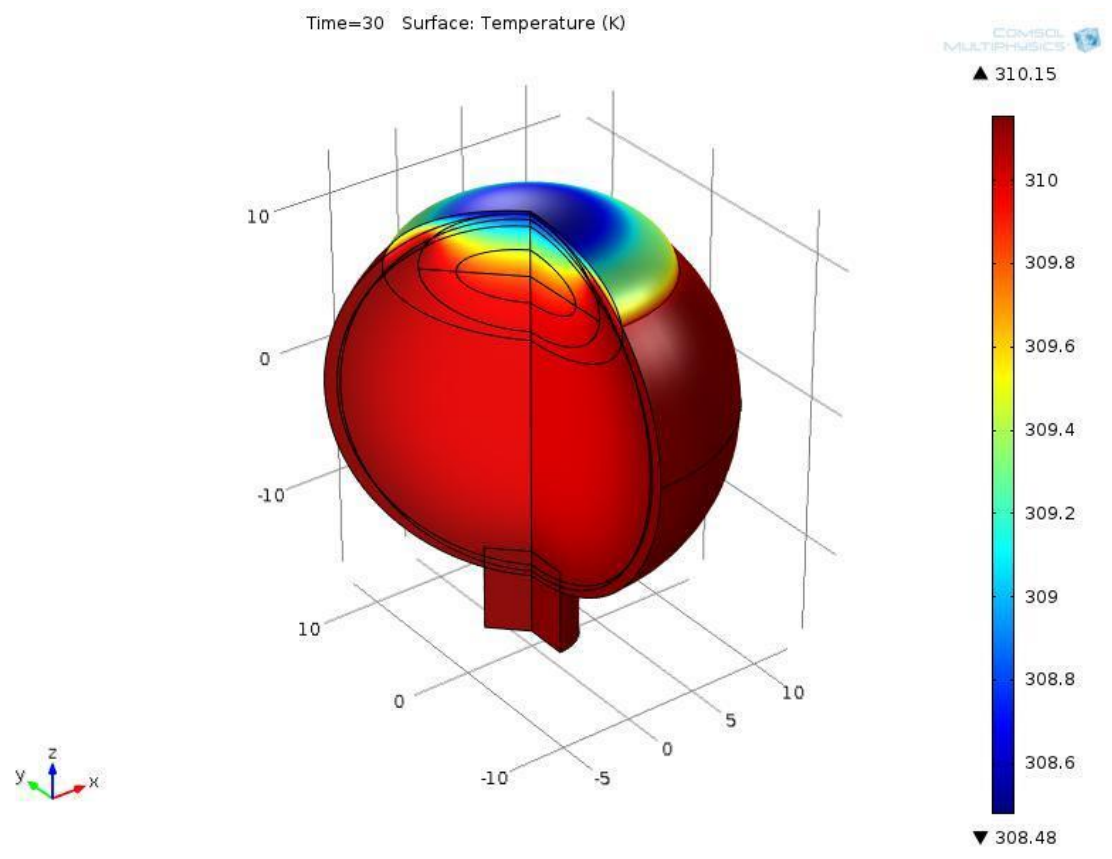

Figure 6.24 3D Temperature Distribution after 30 Second Exposure-PET 


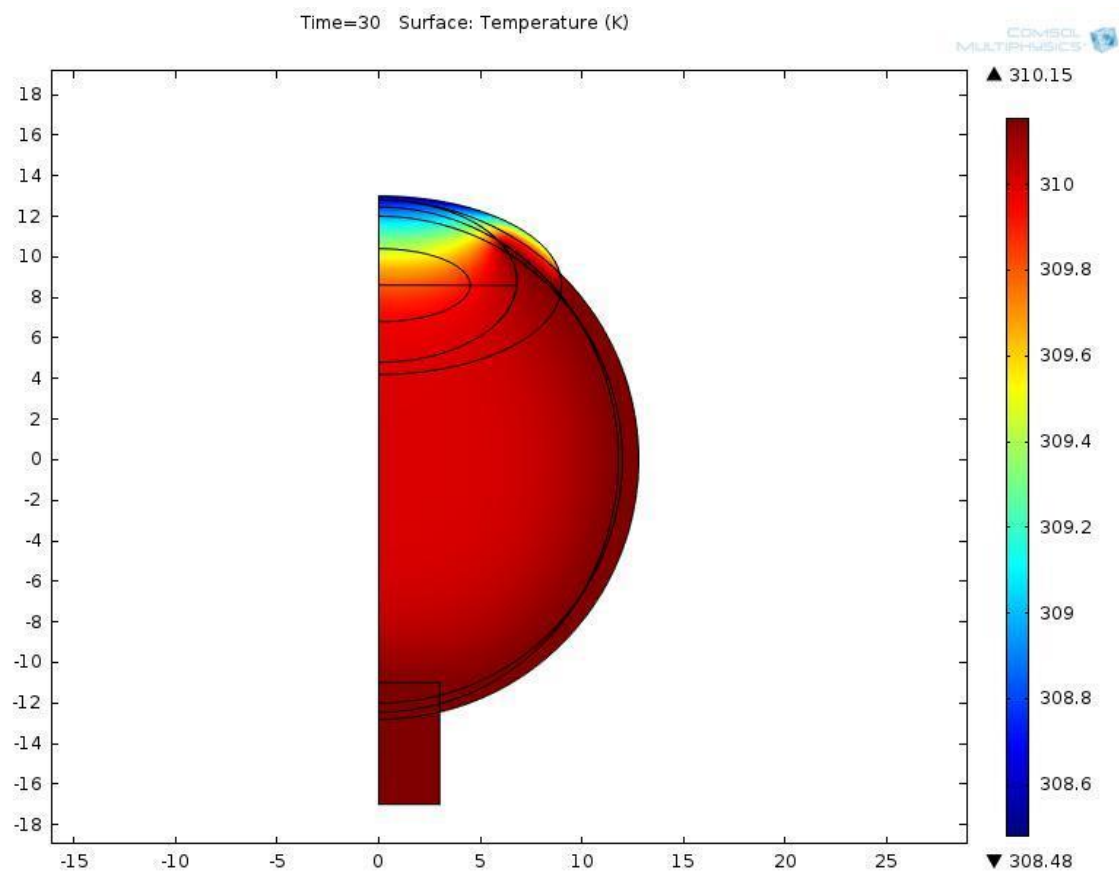

Figure 6.25 2D Surface Temperature Distribution after 30 Second Exposure-PET

For the simulations shown above, PET has a thermal conductivity of 0.24

$\mathrm{W} / \mathrm{m}^{\star} \mathrm{K}$, a density of $1380 \mathrm{~kg} / \mathrm{m}^{3}$, and a specific heat capacity at constant pressure of $1000 \mathrm{~J} / \mathrm{kg}^{*} \mathrm{~K}$. PDMS has a thermal conductivity of 0.15

$\mathrm{W} / \mathrm{m}^{\star} \mathrm{K}$, a density of $970 \mathrm{~kg} / \mathrm{m}^{3}$, and a specific heat capacity at constant pressure of $1460 \mathrm{~J} / \mathrm{kg}^{\star} \mathrm{K}$. What can be determined from the above results is that PDMS is more thermally insulative than PET, and when exposed to the same conditions, causes a higher average temperature throughout the cornea. The gravity of this difference, however, is quite small. The values are within 0.005 degrees Celsius of each other. More importantly, however, is that the above bioheat simulations indicate that even at our maximum allowable power dissipation, the temperature change throughout the cornea is very small, ranging from a 0.5 to 0.7 degrees Celsius increase in both cases. From this it was concluded that from a 
bioheat transfer perspective, both substrates were equivalent and therefore the size is irrelevant, and the power budget for the onboard circuitry was appropriate.

\subsection{Leakage Concerns}

With any biomedical device, biocompatibility is a top priority. Ensuring that the body does not harm the device and the device does not causing harm is an ethical fundamental of engineering. In the case of this research, it applies even more readily since the contact lens is interfaced with a highly sensitive organ. Another primary concern aside from biocompatibility of the system is leakage of particles or chemicals that could get in contact with the eye. The primary particles that were concerning were the electrolytes from the display or the battery and the silver particles from the printed antenna. 


\section{Chapter 7}

\subsection{Applications}

The purpose of the contact lens was to act as a wireless triggered display that would indicate the user that potentially harmful radiation or force was detected. The lens's functionality relies on 4 main components onboard the lens: an antenna for signal and power reception, integrated circuit to drive the display, a battery as the power source, and the display. While the contact lens was the main focus of this project, a test system utilizing an external UV sensor was implemented to demonstrate the intended use of the lens as well as an internal MEMS sensor. The overall system circuitry in the lens was designed to be compatible with other types of sensing mechanisms to give the developer and user a variety of applications that can be tailored to the user's needs. The purpose of this chapter is to explain different applications that were developed during this thesis and future applications that can be developed by the next group.

\subsection{UV Sensor}

An external circuit handles detection of ultraviolet energy. This external system consists of a sensor and wireless transmission circuitry which signals the user during a trigger event. We will refer to the UV index (UVI) to determine the threshold of the trigger event. The World Health Organization recommends the use of sun protection at an index level of 3 [22] - this corresponds to $75 \mathrm{~mW} / \mathrm{m}^{2}$ of radiation. When the sensor detects $75 \mathrm{~mW} / \mathrm{m}^{2}$ of radiation, it will communicate 
to the contact lens by activating the $\mathrm{VCO}$ and transmitting the $2.4 \mathrm{GHz}$ signal. The system block diagram is shown in figure 7.1 below.

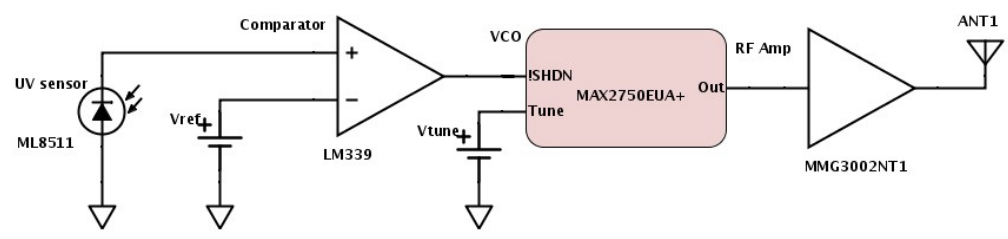

Figure 7.1 External UV Sensor System Diagram

An ML8511 UV sensor by Rohm Semiconductor outputs a voltage ranging from $1 \mathrm{~V}$ to $2.2 \mathrm{~V}$. The output voltage corresponds to both UVA and UVB energies as a total sum. Rohm Semiconductor previously performed field testing in Santa Clara, CA, and the resulting equation was determined to approximate the UVI with the output voltage with an UVI error $+\backslash-$.

$$
U V I=12.49 \text { Vout }-14.735 \text { (for Vout } \geq 1.23 V \text { and } U V I \geq 1 \text { ) }
$$

A non-inverting comparator design was interfaced after the UV sensor that output a logic high value when $1.4 \mathrm{~V}$ was detected from the output of the UV sensor to the positive terminal pin of the comparator. A reference voltage biased the negative terminal pin at $1.3 \mathrm{~V}$ using a resistor divider to create this threshold tolerance. This can be seen below in Figure 7.2.

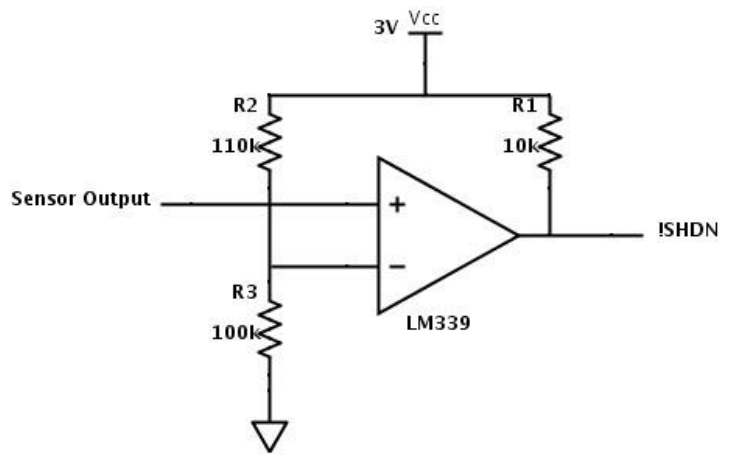

Figure 7.2 Comparator 
The output of the comparator drives the input pin of the voltage controlled oscillator (VCO) where a logic high value of $3 \mathrm{~V}$ on the $S H D N$ pin will activate the VCO taking it out of low-power shutdown mode. The last stage involves amplifying the output signal in order to fulfill the $32 \mathrm{~cm}$ operational distance requirement. The MMG3002NT1 by Freescale Semiconductor was chosen for its high $1 \mathrm{~dB}$ compression point of $21 \mathrm{dBm}$ and is internally matched to $50 \Omega$ impedance.

Essentially what is happening is when $75 \mathrm{~mW} / \mathrm{m}^{2}$ of radiation hits the sensor, the output of the comparator swings high and the VCO outputs a $-3 \mathrm{dBm} 2.4 \mathrm{GHz}$ signal. The signal generated from the VCO is amplified by the amplifier that has a $\left|S_{21}\right|$ of $16 \mathrm{~dB}$ at $2.4 \mathrm{GHz}$ as listed in the datasheet, which would boost the output signal of the VCO to $13 \mathrm{dBm}$ maximum.

The first portion of the testing confirms the operation of the UV sensor. The output of the comparator swings high right when the input sees $1.425 \mathrm{~V}$. This results in the comparator turning on the VCO when the sensor detects around 3.4 UVI which is comparably higher than the original UVI level of 3 trigger point. It was concluded that the resistor tolerances were the cause which increased the threshold point of the comparator. The 0.4 UVI trigger point error, however, could be deemed negligible from the calculation of equation 7.1 that produced a UVI error of $+\backslash-1$. The VCO used was the MAX2750 which has a tunable frequency range of $2.4 \mathrm{GHz}$ to $2.5 \mathrm{GHz}$ even though operation only requires a single frequency. The VCO was tested with the MMG3002NT1 RF amplifier in the output stage. The voltage for $V_{\text {tune }}$ must first be obtained such that the output of 
the VCO is $2.4 \mathrm{GHz}$. Since the VCO's output frequency is linearly proportional to $\mathrm{V}_{\text {tune }}$ and the output frequency when $0 \mathrm{~V}$ is applied to $\mathrm{V}_{\text {tune }}$ is $22.5 \mathrm{GHz}$, the voltage needed for $2.4 \mathrm{GHz}$ operation was calculated to be $0.53 \mathrm{~V}$.

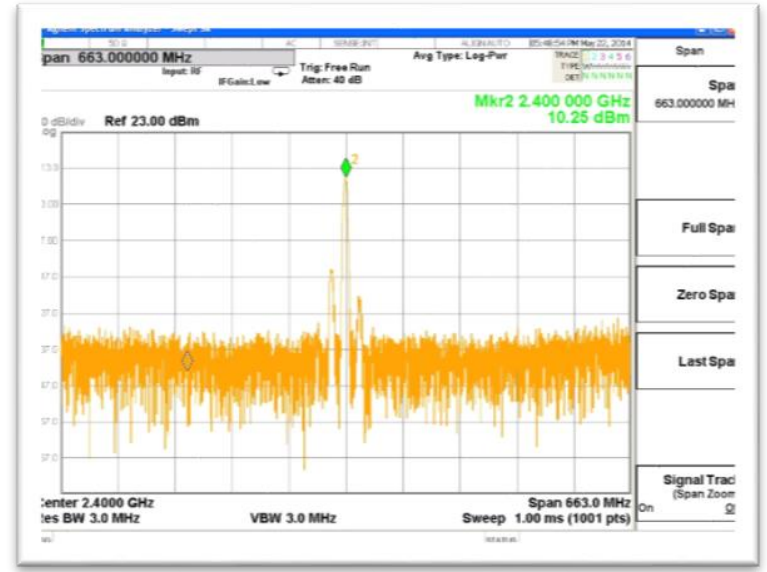

Figure 7.3 VCO output with $0.53 \mathrm{~V}$ at VTUNE

Figure 7.3 shows when $0.53 \mathrm{~V}$ was applied to $\mathrm{V}_{\text {tune }}$ a frequency of $2.4 \mathrm{GHz}$ is being output. The spectrum analyzer shows an output power of $10.25 \mathrm{dBm}$ which is about $3 \mathrm{~dB}$ lower than the estimated maximum of $13 \mathrm{dBm}$. The reason behind the power loss was because the $13 \mathrm{dBm}$ maximum output assumes no load condition while the input to the spectrum analyzer is $50 \Omega$. The input resistance of the spectrum analyzer creates a voltage divider with the $50 \Omega$ of the RF amplifier which drops the output of the RF amplifier by $-6 \mathrm{~dB}$. Therefore, the expected output power should be roughly $7 \mathrm{dBm}$ which is lower than the measured result in Figure 7.3. The $10 \mathrm{dBm}$ output power from the antenna should be high enough for the contact lens to detect the signal $32 \mathrm{~cm}$ away.

\subsection{Wireless Charging}

A fixed on-site thin film battery provides power to the IC in the contact lens. In order to recharge the battery, the use of induction charging was 
employed. This was determined as the best solution for power transfer because the charging is done off the eye in a contact lens case. The idea was the user would take off their lenses before going to sleep and in the morning the lenses would be fully charged. From a prior project, an inductive charging unit was implemented to recharge an onboard battery in a flexible time apparatus. The charger used a $13.56 \mathrm{MHz}$ frequency which is the same frequency used to charge the lens. This section will describe the functional application of inductive charging and reference work from another group project that I took part in.

\subsubsection{Background}

An inductive charging system is composed of two coils for wireless energy transfer and a rectifier to convert the $\mathrm{AC}$ signal to a $\mathrm{DC}$ signal. $\mathrm{A}$ pair of coils provide wireless energy transfer from the transmission side to the receive side by employing Ampere's and Faraday's law. A time varying current drives the transmission coil which by Ampere's law creates a time-varying magnetic field. The magnetic field induces a voltage onto the receiving coil due to Faraday's law. The plan was to place the coils close to each other during the charging phase thereby allowing a simplified coil design by creating matched coils. Coil design and resonance was not critical at that stage because we did not plan on wireless charging at a long distance which allowed us to work with higher frequencies and smaller coils. 
The coil's impedance is compared through theoretical computations and a printed prototype. The coil was designed to fit the size of an ID-1 card and was a rectangular shape instead of circular. The table below shows the parameters of the coil.

\begin{tabular}{|c|c|}
\hline $\begin{array}{c}\text { \# of Turns } \\
\text { Inner Radius } \\
\text { (mm) }\end{array}$ & 25 \\
$\begin{array}{c}\text { Trace Width } \\
\text { (mm) } \\
\text { Spacing Width }\end{array}$ & 1 \\
$\begin{array}{c}\text { (mm) } \\
\text { Table 5 First Iteration Coil Parameters }\end{array}$
\end{tabular}

The model was drafted in ADS and S11 impedance was simulated for a small range of frequencies. The results are seen below in Figure 7.4.

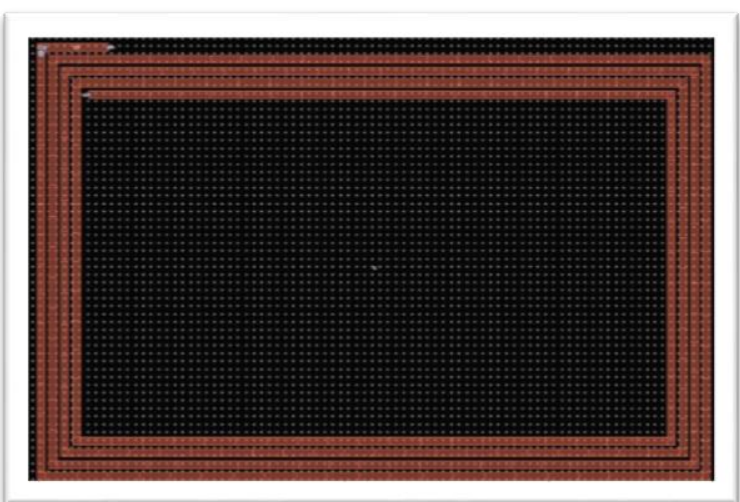

Figure 7.4 ID-1 Sized Coil 


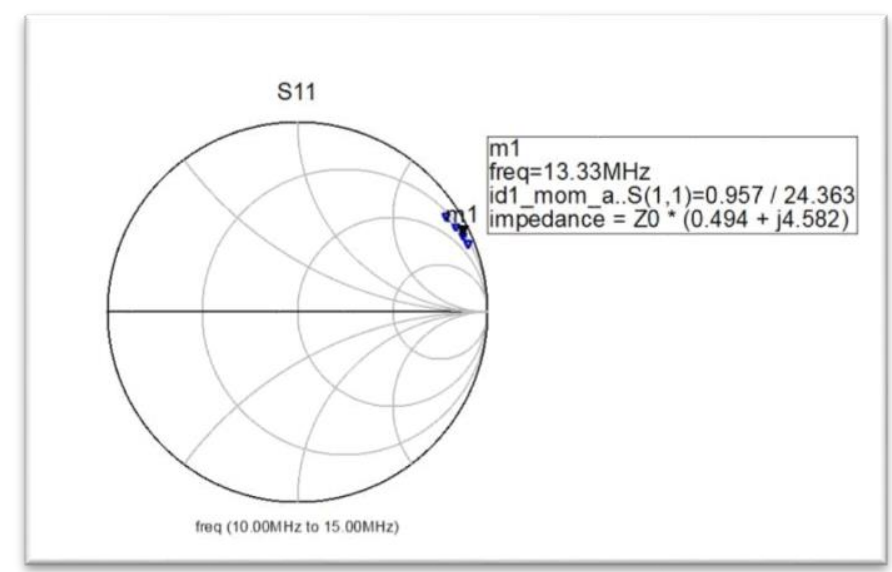

Figure 7.5 Simulation Results of Printed Coil

The inductance calculated from the smith chart is:

$$
\text { Inductance }=\frac{X_{L^{*}} Z 0}{2 \pi * \text { frequency }}=\frac{4.582 * 50}{2 * 3.14 * 13.54 \mathrm{MHz}}=2.74 \mu \mathrm{H}
$$

Simulations show an inductance of $2.74 \mu \mathrm{H}$. The design was then translated to illustrator and a mask is printed. The steps after printing the mask are the same as the printed antenna or electrochromic display for making a patterned screen and printing on PET.

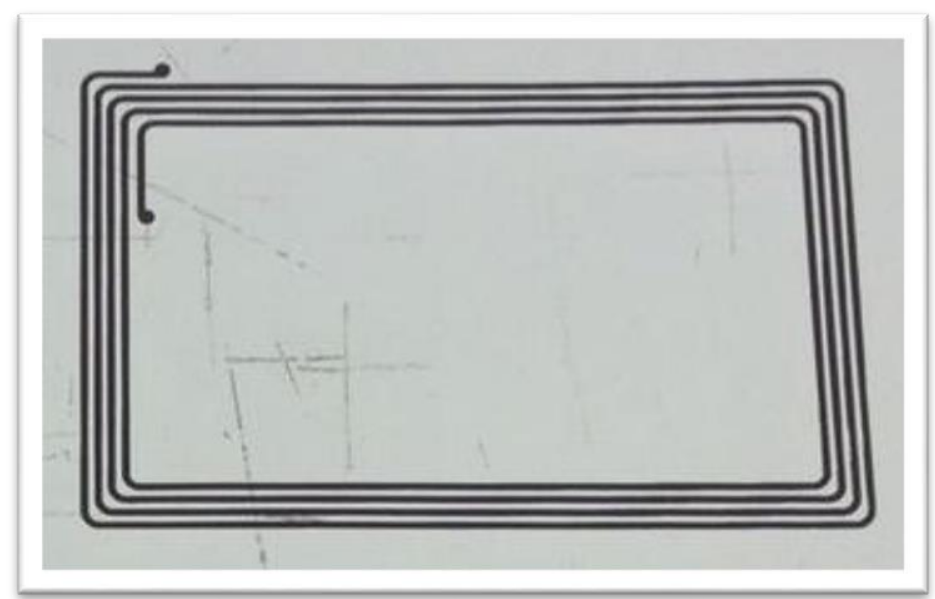

Figure 7.6 Printed ID-1 Coil 
The measured inductance is $2.43 \mu \mathrm{H}$ giving a percent error of $13 \%$. The figure below confirms wireless energy transfer of the Tx and Rx coils.

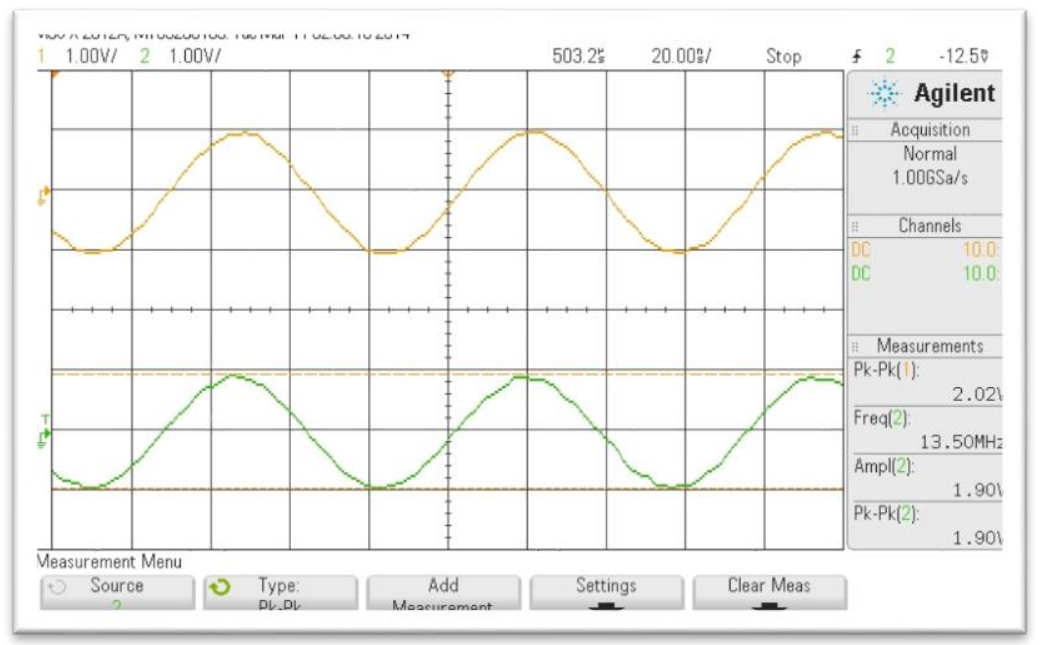

Figure 7.7 Oscilloscope Capture of Tx Signal (top) and Rx Signal (bottom)

\subsubsection{Rapid Prototyping a Charging Case}

The 3D printing method used is called Fused deposition modeling (FDM) and works using a plastic filament (or even metallic wire) which is taken from a coil and supplies material to an extrusion nozzle (similar to a common printer) which turns the material/ink flow on and off. The nozzle is heated to melt the material and can move controlled by a special software package. The object is produced by extruding melted material to form layers on top of each other as the material hardens immediately after extrusion from the nozzle. The machine dispenses two different materials, one is the model material for the final object you want to have printed and the other is the support material that is a disposable support structure. 
For this specific case, the actual printing process took about seven hours for the machine to create all of the layers of the box and an additional 4 hours were needed for dissolving and washing out the support material. Figure 7.8 shows the finished 3 dimensional model.

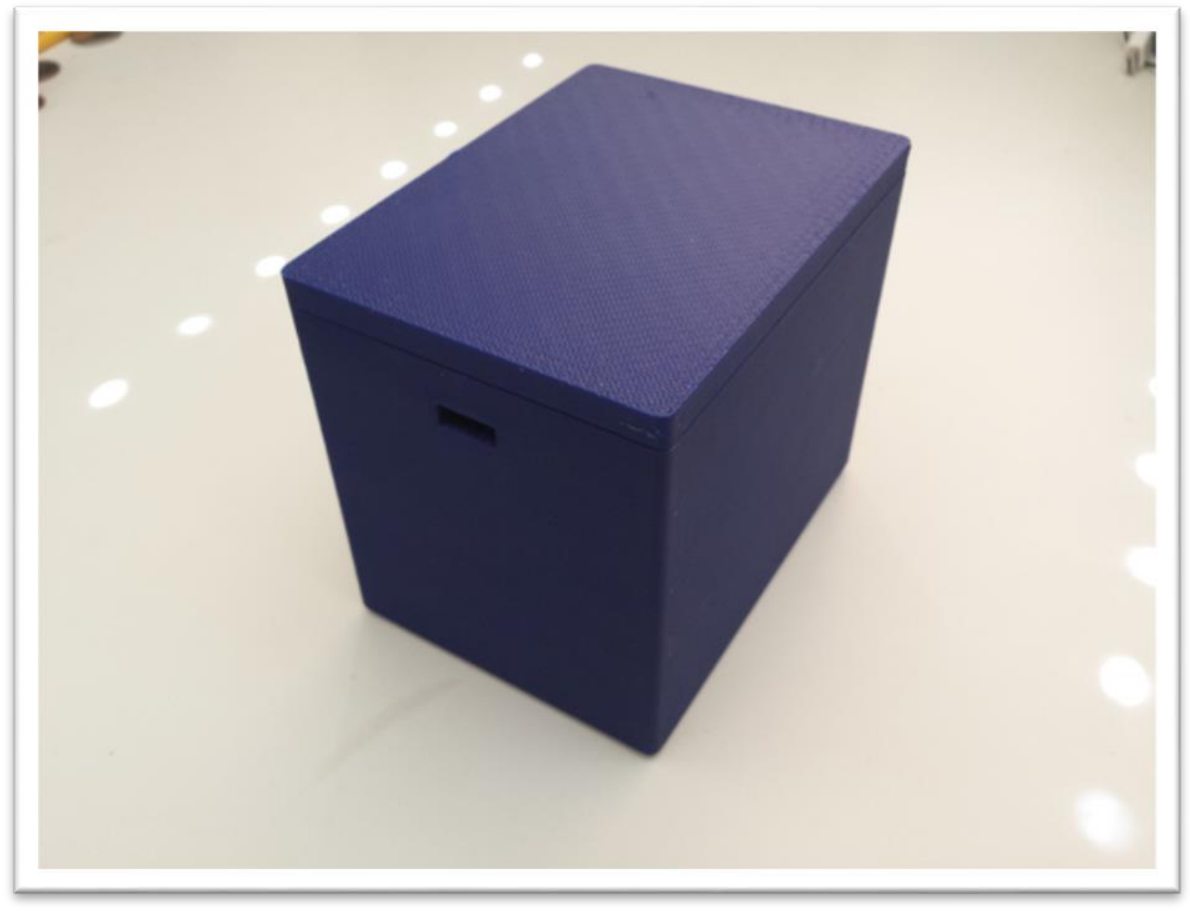

Figure 7.8 Rapid Prototyped Box Case for Inductor Transmit Coil The transmission side of the charging system produces the electromagnetic field for the receive side. The charging circuitry is mainly an oscillator that takes a DC voltage to generate a sinusoidal waveform. We designed the oscillator to have a $5 \mathrm{~V}$ supply to support both the use of batteries and the ability to be powered via USB. The circuit that drives the transmitting coil is an oscillator that utilized a tank circuit with negative resistance shown in Figure 7.9. To implement a more stable oscillator a phase-locked loop (PLL) would be a better alternative to the Vackar Oscillator. 


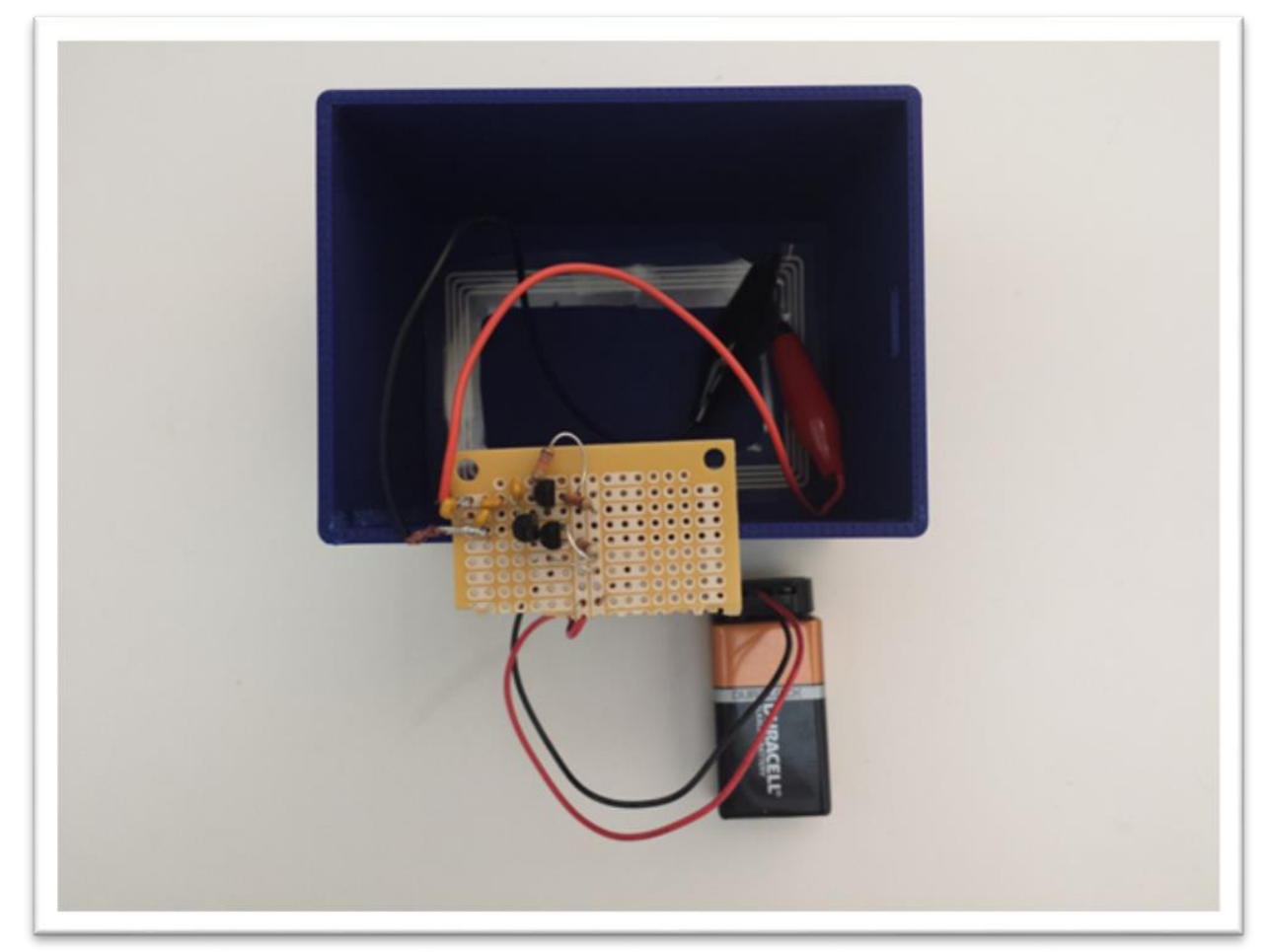

Figure 7.9 Inductor Coil with Driving Circuit and Power Source

\subsection{Strain Gauge}

The main technology that was used to measure changes in intraocular pressure (IOP) is classified as a micro electric mechanical system (MEMs). These systems can be characterized as miniature mechanical and electro-mechanical devices that exist on the nano-scale and are produced using various techniques in micro-fabrication [23]. These machines can be used for a variety of purposes including but not limited to accelerometers, light sensors, and pressure sensors. In this application, MEMs were used to detect slight changes in the surface curvature of the eye with the use of a strain gauge. Leonardi et al. had developed a methodology for integrating strain gauges such as the one 
proposed into a contact lens, in order to detect slight changes in IOP as it is observed through the sclera as a change in the surface curvature. MEMs devices detect changes in the environment and use a specific type of impedance to communicate this change to an integrated circuit. In the case provided, the MEMs strain gauge uses resistance impedance, which can then be converted into a change in pressure of the aforementioned eye [24].

The development of this device had several challenges in both the design and manufacturing process. This section discusses implementation and fabrication of a strain gauge that is sandwiched between two micro-thin layers of PDMS used for insulation. The strain gauges measured at $50 \Omega$, and were tested with a Wheatstone bridge to determine if the device changes its resistance due to strain. It was found that the strain gauge resistance showed minuet change when bent. Unfortunately, there was a lack of accurate instruments to fully measure and properly characterize the device resistance change since the changes were in the $\mu \mathrm{V}$ range with the Wheatstone bridge.

\subsubsection{Background}

The rise of glaucoma patients has concerned many optometrists, where optometrists need better methods of monitoring glaucoma than what current technology offers. Optometrists need to continuously monitor glaucoma so as to accurately prescribe the correct medication for each patient. However, IOP can change throughout the day [25], and so patients 
must either see their optometrists daily or be monitored in a 24-hour monitoring system at the optometrists' offices. A daily visit can be inaccurate due to the IOP changing constantly throughout the day, and staying at an office for 24 hours is too time consuming for patients. Since optometrists need to constantly monitor patients' IOPs, a MEMS device was needed to be designed, fabricated, and embedded on a contact lens that can constantly monitor patients' IOPs.

The eye is one of the most complex and sensitive organs in the human body, second only to the brain. The eye is separated into two main segments, the anterior and posterior seen in Figure 7.10.

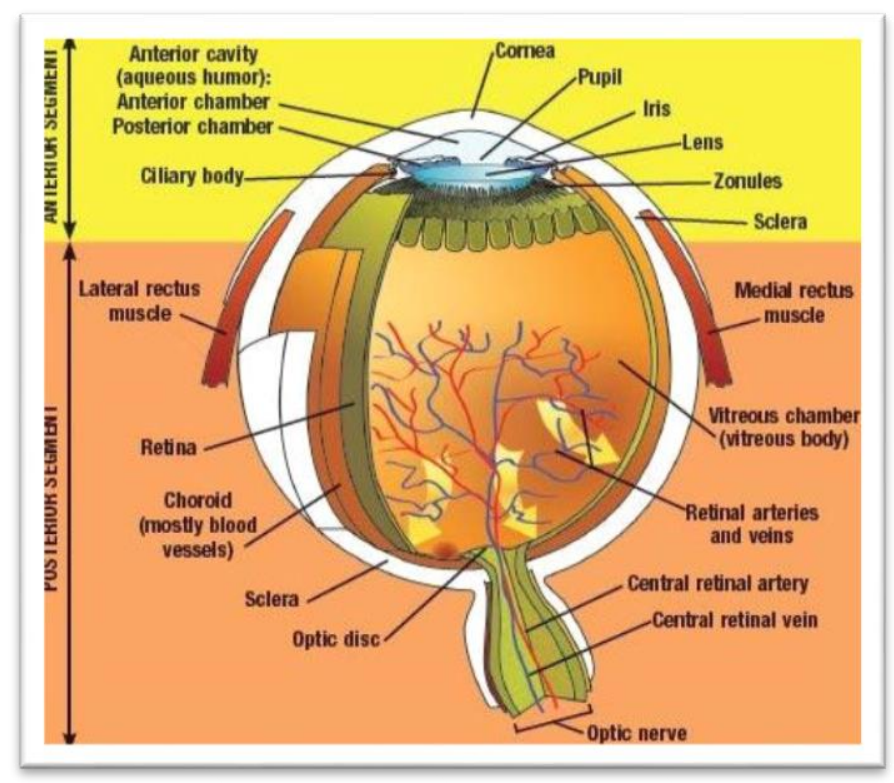

Figure 7.10 Anatomy of the Eye [26]

The anterior segment is identified as the part of the eye that takes in optical stimuli from the environment whereas the posterior segment focuses on the translation of these optical stimuli into chemical and electrical signals that can be transferred to the brain through the optical nerve. Each 
component of the eye is sensitive to slight changes in chemical and physical imbalance; one of the most common is when there is a large fluctuation in the IOP. IOP is the overall pressure experienced in the eye and is controlled by three main chambers in the eye: the vitreous chamber (body), and the anterior and posterior chambers of the anterior cavity. Aqueous humor is regularly cycled through the anterior cavity to maintain a constant environment for optimal vision seen in Figure 7.11.

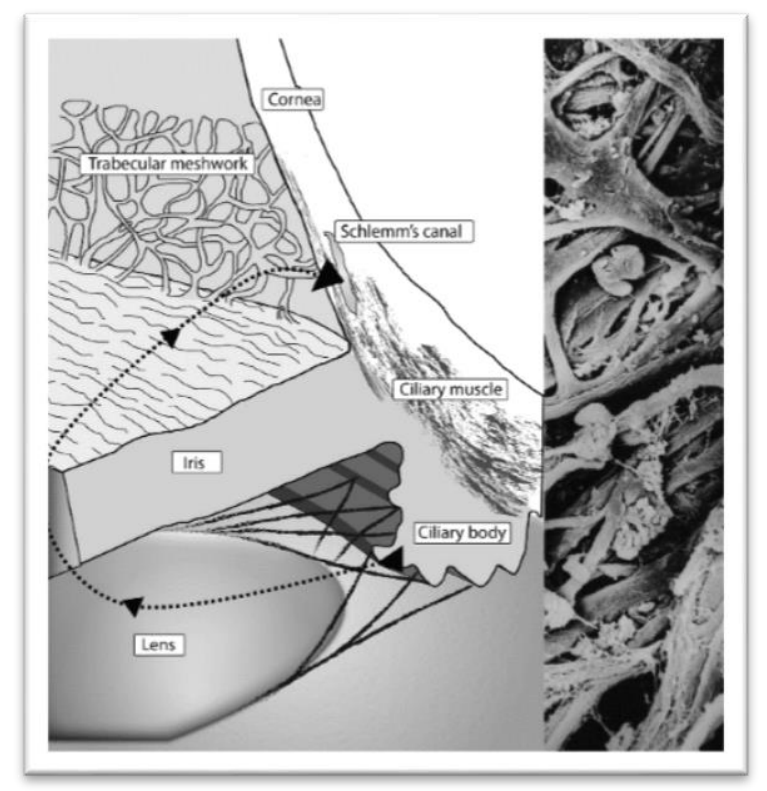

Figure 7.11 Flow pathway of aqueous humor through anterior chambers [26] A change in the rate of aqueous humor production or the rate at which the humor is drained from the anterior chambers will change the overall IOP. This fluctuation in pressure is a common symptom to a prevalent disease known as glaucoma. Glaucoma is one of the leading causes of blindness around the world and in 2010 an approximate 60.5 million people experienced symptoms of glaucoma [27]. Glaucoma results from an increase of the average IOP experienced by the patient, which in turn 
causes damage to the retina and optic nerve and progressively damages the patient's field of vision until complete blindness ensues seen in Figure 7.12. A less common form of glaucoma is caused by a decrease in the IOP, which leads to a detachment of the retina from the rear of the eye.

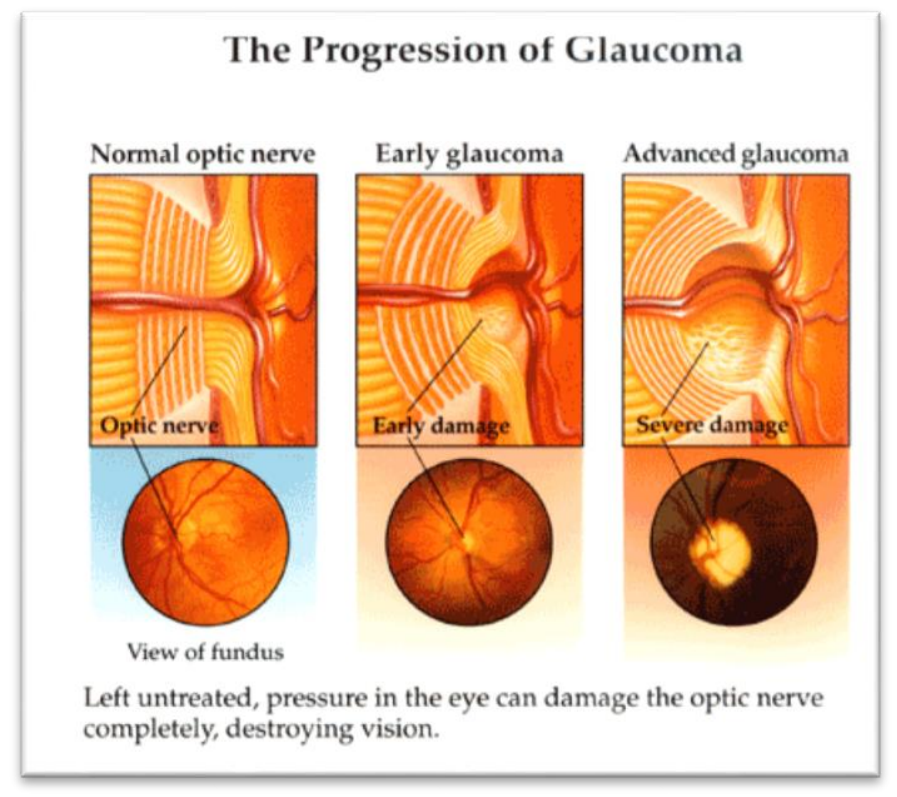

Figure 7.12 Progression of high-pressure glaucoma [28]

Currently Goldmann Applanation Tonometry is the gold standard in eye care for determining the IOP of a patient. This procedure numbs the eye and applies a constant pressure to the cornea of the eye. The data collected from the force measurement applied can be linearly related to the IOP. Many technologies in use operate on the same general principle that drives the Goldmann procedure [29]. Although these types of instrumentation are commonly used in practice, they are limited to measuring the average value of a patient's IOP at a set instant in time, thus far there has not been a commercial device with the capability to collect pressure data from a patient over the span of time. The IOP 
values for any person will vary as the day progresses depending on posture, activity, the environment and multiple other stimuli, many of which have not been fully determined [30]. The ability to monitor the IOP of a glaucoma patient over long periods of time allows for the patient and the physician to have a better understanding of the magnitude of variability in the IOP, the actions that most affect the fluctuations, and to determine the best course of action in terms of medication or preventative measures for the disease.

\subsubsection{Research for Strain Gauge}

The group I lead for this device researched three different designs for the monitoring system: the micro-cantilever, the strain gage, and the piezoelectric designs. These designs have previously been used for sensing pressure; the structure designs can either bend or deflect due to different pressure levels applied to the structures.

Three different designs were researched for the monitoring system: the micro-cantilever, the strain gage, and the piezoelectric designs. These designs have previously been used for sensing pressure; the structure designs can either bend or deflect due to different pressure levels applied to the structures.

The strain gauge measures IOP indirectly by measuring the curvature changes on the sclera. The strain gage design is a thin loop of material that can change shape without breaking apart (i.e. gold and silver) [31]. Figure 7.13 shows an example strain gage, where the loop changes its 
curvature according to the sclera's curvature.

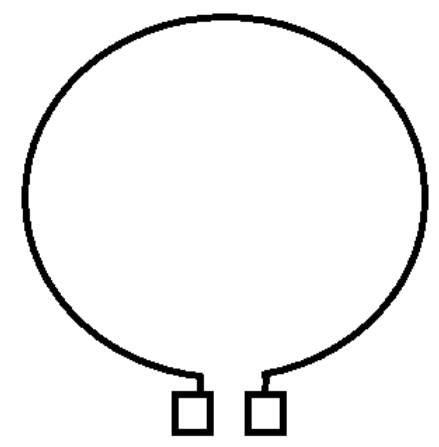

Figure 7.13 Example Strain Gage Design

The piezoelectric design measures pressure by the eyelids and sclera applying pressure onto the piezoelectric material. Depending on the design, this piezoelectric material changes either its resistance or capacitance. The piezoelectric material changes its resistance or capacitance whenever it experiences a mechanical stress [32]. Since the strain gauge has already been used before on a contact lens [31], it was decided to use this design over the other two designs because for lack of materials on campus as well as less room for error in fabricating. For example, while micro-cantilevers have been a wellestablished MEMs device used for many years, the fabrication process for micro-cantilevers require several masks, which would need to be created. Due to time constraints and multiple chances of error in manufacturing, the micro-cantilever design was disregarded. Similarly, the piezoelectric design also requires several masks as well and multiple processing stages of doping, sputtering, and edging. The strain gauge ideally needed only two masks if an adhesion layer is needed and one 
mask if not. The process only required sputtering which made it simple to manufacture. This was the deciding factor for the strain gauge design.

\subsubsection{Design and Fabrication of Strain Gauge Molds and Masks}

The strain gauge consisted of either gold or silver; each material showed different characteristics for curvature, resistivity, and adhesion on the PDMS layer. The bottom PDMS layer was cast from an acrylic mold as well as the mask for patterning the gold or silver onto the bottom PDMS layer. The acrylic mold and mask were fabricated by designing a CAD model that could be used for laser-cutting the acrylic. This model shows the mask of the strain gauge and mold for the bottom PDMS layer. The model file was then uploaded to a laser-cutting machine, Versa Laser, that resulted in Figure 7.14 below.

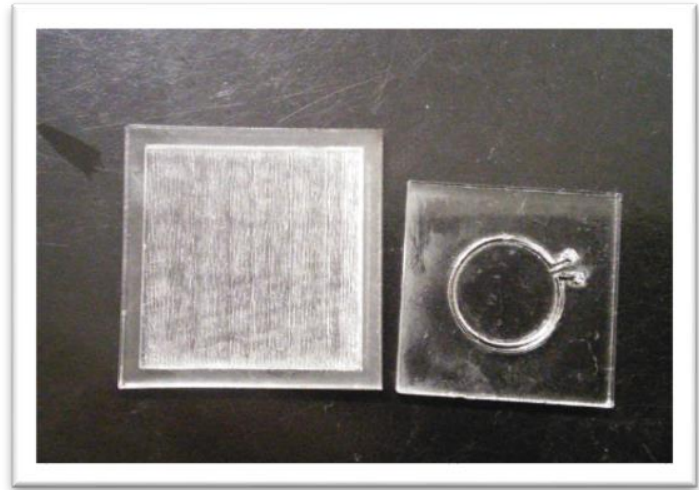

Figure 7.14 Strain Gage Mold (left) and Mask (right), Laser-cut from PET

To create and cure the PDMS onto the molds, I used the following procedure for curing PDMS:

1) Check the level of the PDMS curing oven located in Nano Lab.

2) Set the PDMS curing oven to a temperature of $70^{\circ} \mathrm{C}$.

3) Monitor oven temperature using available thermocouple 
4) Determine total quantity (volume) of PDMS necessary to produce a thin film (100 microns thick) square in an acrylic model.

5) The formulation used for mixing the solvent was 10:1 (base : curing agent) mix ratio.

6) The mixture was stirred thoroughly and placed in the Degas Chamber where the solution is vacuumed and vented.

7) When closed the vacuum pump is turned in utility chase. The vacuum line valve is opened and pumped down until large bubbles ascend to the surface and pop. Throttle the chamber pressure using the vent valve so that PDMS doesn't overflow cup.

8) Close vacuum line valve, slowly vent chamber, and remove the degassed PDMS solution.

9) Slowly pour degassed PDMS into the empty models and place in the curing oven for 2 hours.

The cured PDMS appears in Figure 7.15, with the PET masks on top.

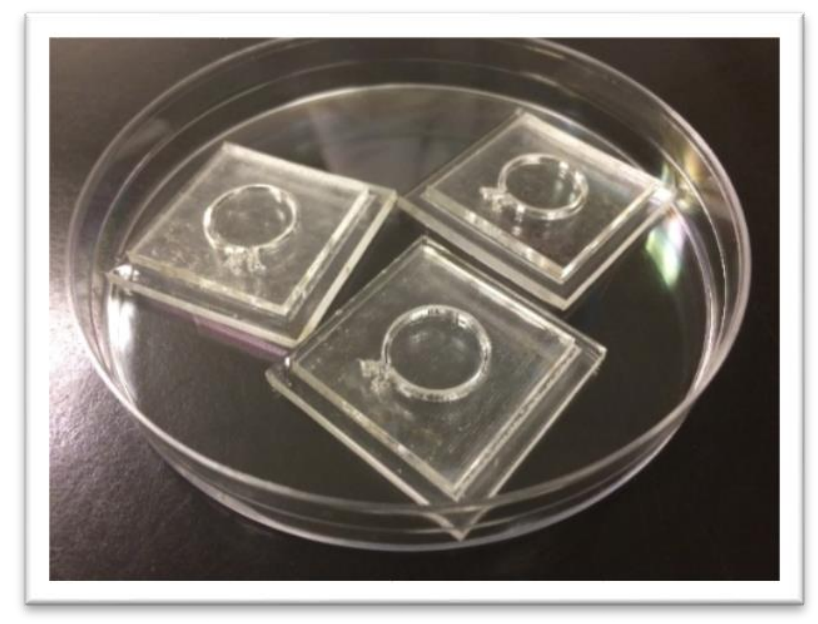

Figure 7.15 Cured PDMS on Molds

Once the PDMS was cured in the mold, the next step in the process was

to sputter the gold/silver creating a thin film on the mold. The following procedure was used for sputtering:

6) The following gas tanks were turned on: High Pressure Argon, High Pressure Nitrogen, and Low Pressure Nitrogen gas.

7) The molds were placed inside the sputtering machine, the Denton Vacuum, as seen in Figure 7.16. 


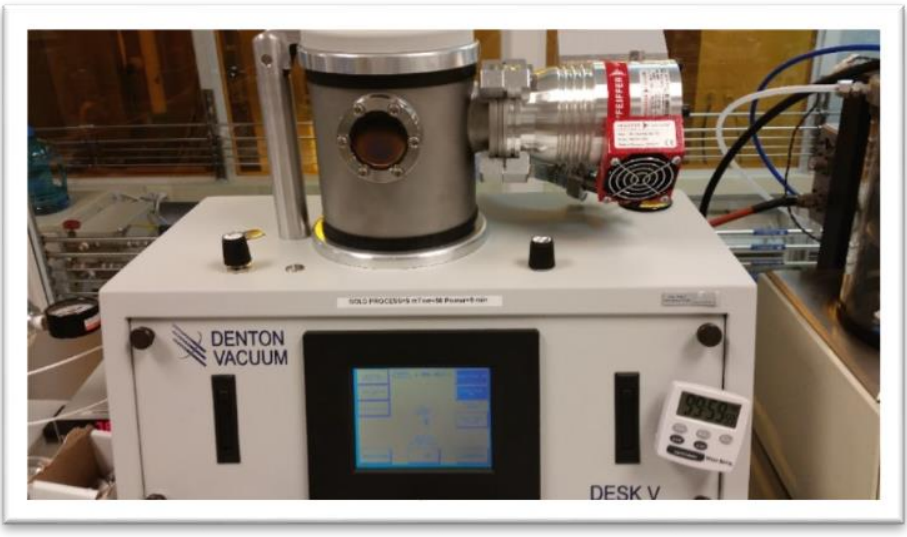

Figure 7.16 Denton Vacuum PVD Machine

8) The gold target was installed in the machine as seen in Figure 7.17 .

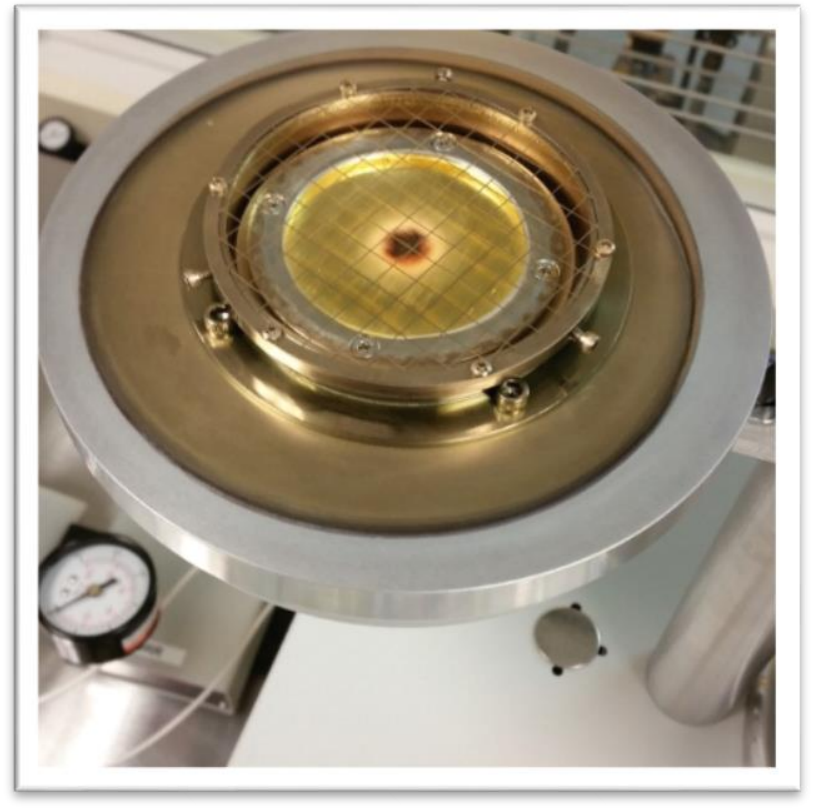

Figure 7.17 Gold Target for Sputtering 


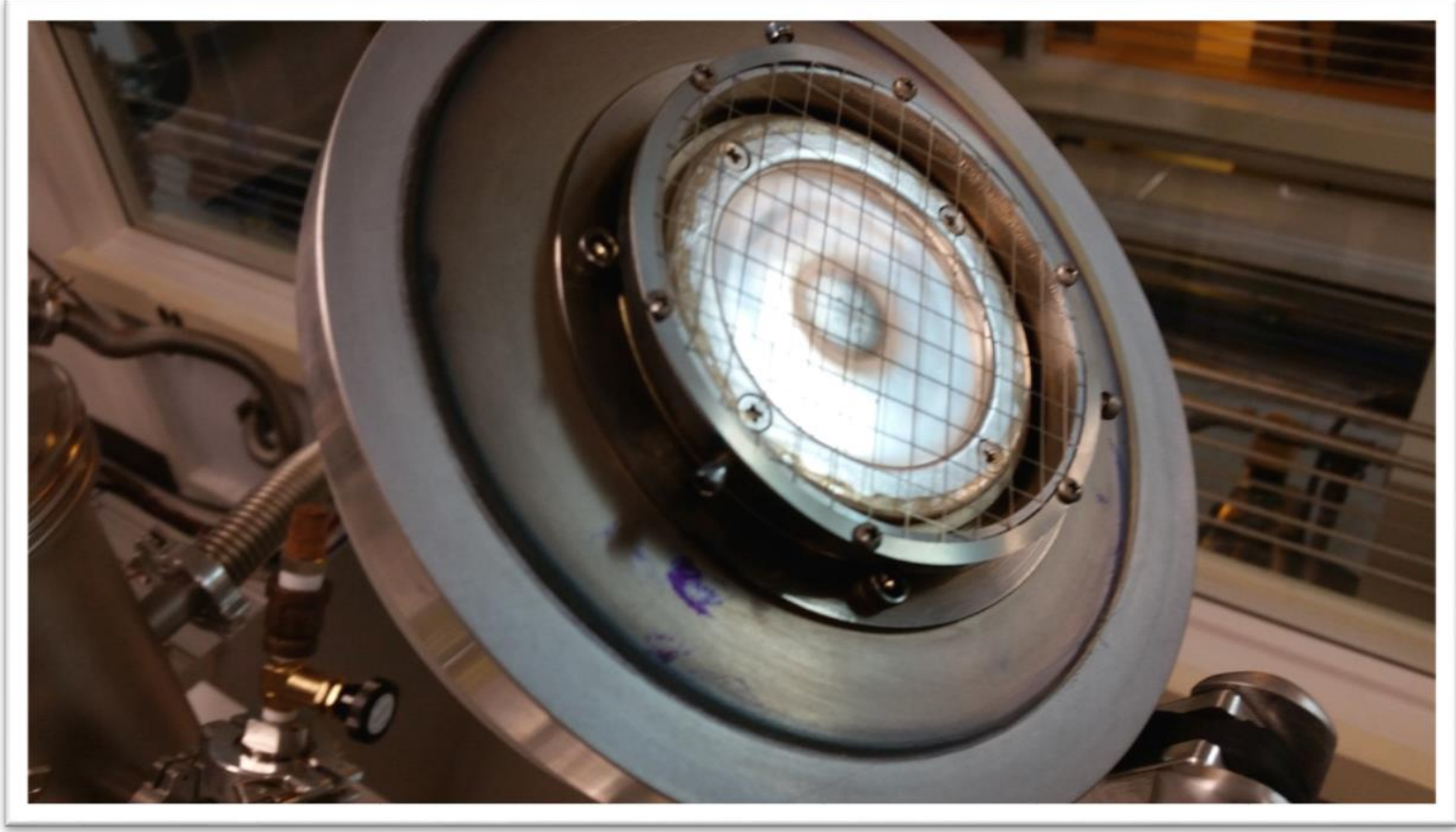

Figure 7.18 Silver Target for Sputtering

9) After installation the machine was turned on.

10) The cured models were sprayed with nitrogen and placed in the chamber with a glass slide and a piece of tape to attach to the bottom of the rotating target platform.

11)Pressed the start menu on the touch screen and turned on the mechanical pump.

12) Waited until the pressure was at .12 torr to activate the turbo pump.

13) Wait until base pressure reached $1.90 * 10^{-5}$ torr which took about one hour and 45 minutes.

14) Turned on the gas value resulting in pressure jump of 9 mtorr.

15) Wait 5 minutes and initiate rotation.

16) Turned on sputter power (on display, the DC Current reads 48 $m A)$.

17) Time of sputter was approximately 7 minutes.

18) Turned off sputtering and rotation and auto vent to stabilize pressure to atmospheric pressure. This step has a 600 second count down and an additional 5 minute wait time for venting.

19) The gold target was replaced out and the silver target was installed.

20)Steps 4-13 were repeated.

Figures $7.19,7.20$, and 7.21 show the results of sputtering. 


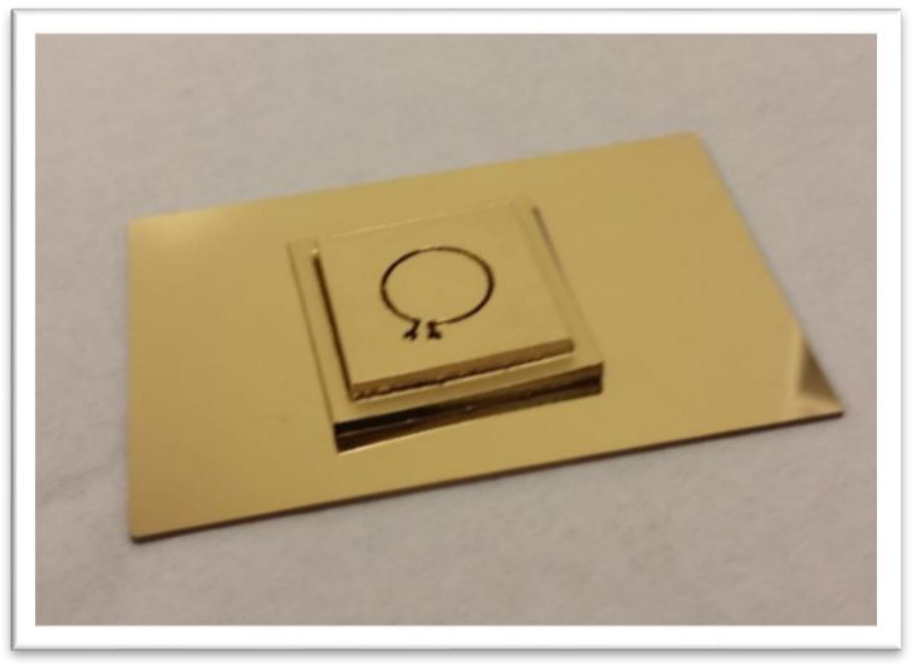

Figure 7.19 Gold Sputtered Mask and Mold

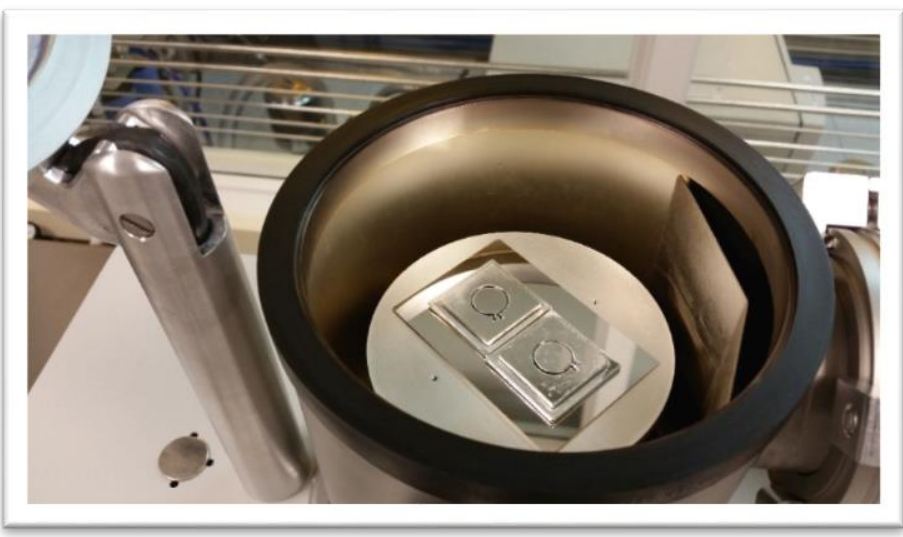

Figure 7.20 Silver Sputtered Mask and Mold

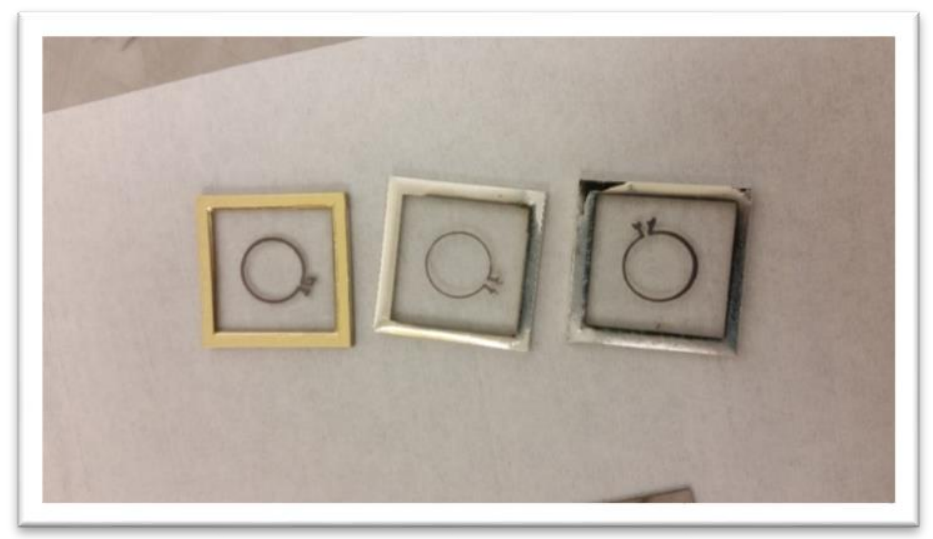

Figure 7.21 Gold and Silver Strain Gages, First Production 
The resulting strain gauges seen in Figure 7.21 were fabricated. It was speculated that there were discontinuities in the strain gauges due to the thickness of the mask resulting in a thickness less than the desired range of $170-200 \mathrm{~nm}$. Also, it was unsure if silver and gold would stick to the PDMS without an adhesion layer of titanium. Testing of the first production confirmed my theory of the strain gauges failing the continuity test and were placed under an optical microscope to observe microfractures.

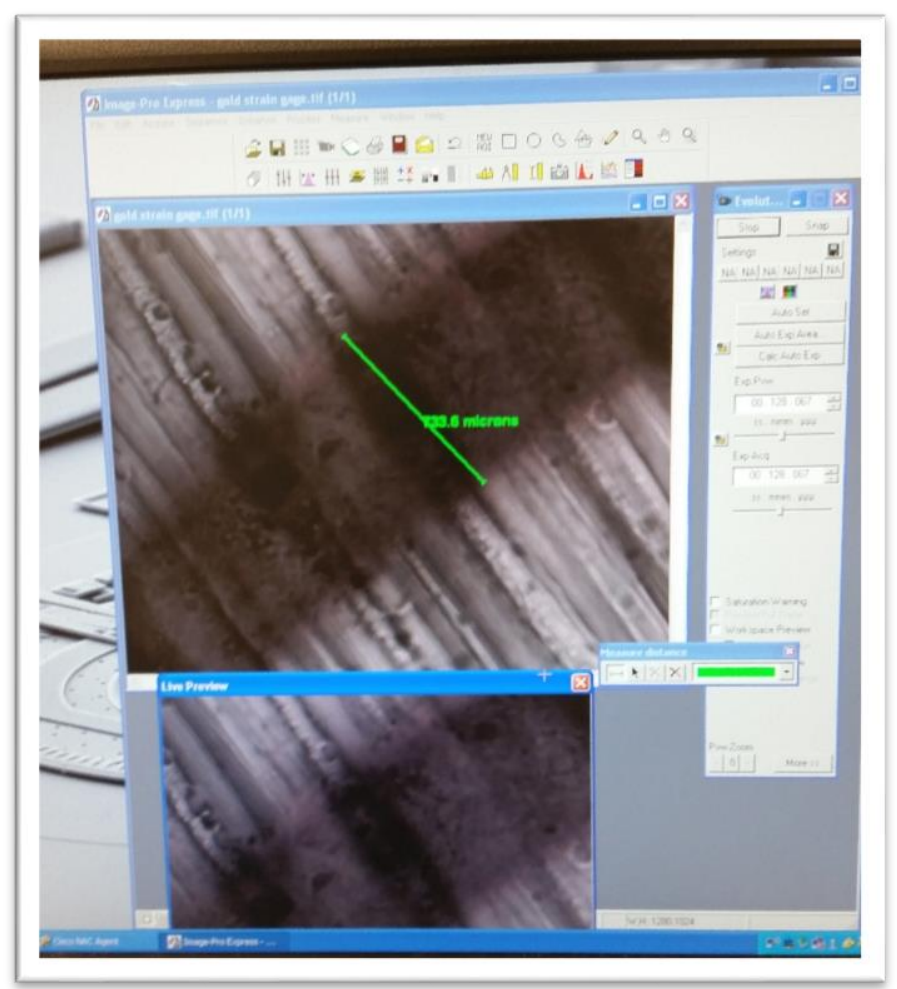

Figure 7.22 Microscope Capture of Strain Gauge Gold Sample It was also discovered that there was contamination on the silver traces that had sections of tarnish and oxidation. This drastically increased resistivity which resulted in a very high impedances. 
To solve these matters, the masks that were sputtered in the first trial were tested for continuity which it had. This meant that the likelihood of the silver/gold atoms from was less than it hitting the sides of the mask. I redesigned and re-fabricated the mask on a 25 micron PET substrate using a die cutter that outlined and the cut traces. The mask had a diameter of $1.4 \mathrm{~cm}$. The steps for curing PDMS and sputtering gold/silver were implemented again and bared successful results with the desired thickness, continuity, and an impedance value close to $50 \mathrm{ohms}$. The silver gauges were measured to have a resistance of $49.9,50.3 \mathrm{ohms}$, the gold gauge was too thin after fabrication and registered as an open circuit when tested. After gauge testing was completed the sensors were encapsulated in PDMS to reduce contamination factors. Figure 7.23 shows the second fabrication trial of strain gauges.

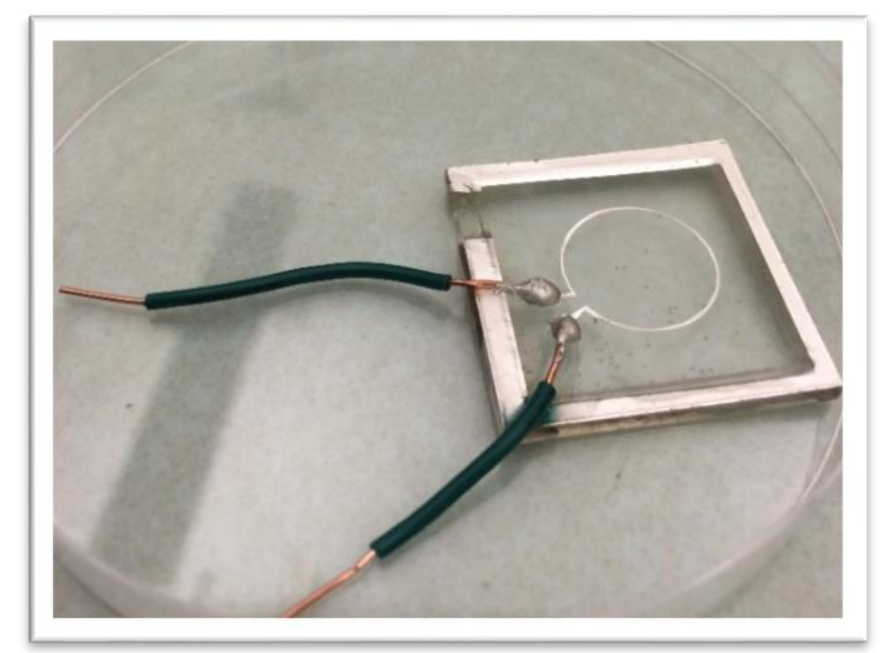

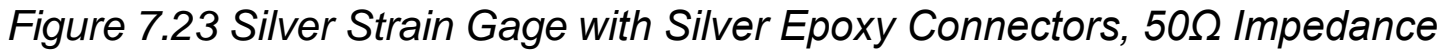

\subsubsection{Data Results}

When the curvature of the eye changes due to a change in IOP the strain gauge experiences a strain which changes the resistance of the gauge. 
To test our strain gage, a Wheatstone bridge circuit was used to detect the change in resistance of the gauge due to changing IOP. The Wheatstone bridge configuration shown in Figure 7.24 was used with one active gauge and one passive gauge in addition to two discrete resistors. The discrete resistors as well as the passive and active gauges were well matched in their resistances so that under zero strain conditions the voltage $V_{m}$ would be close to zero.

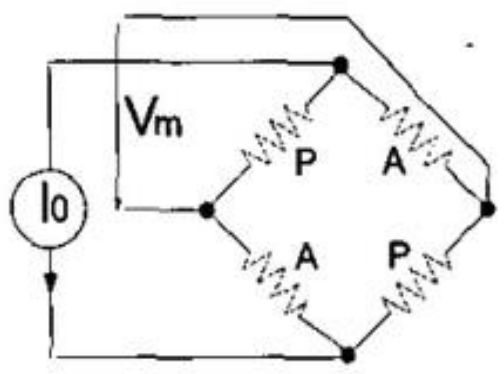

Figure 7.24 Wheatstone Bridge Circuit, Active and Passive Resistors In order to test the accuracy and reliability of the strain gauge in measuring IOP, tests were carried out on the latex eye model. Using the test setup shown in Figure 7.25, an air pump was fed into an IV bag filled with saline solution and was used to pump the solution into the model eye. The contact lens containing the strain gauge was placed on the model eye to measure changes in pressure. The pressure gauge on the air pump was used as a comparison against the values measured from the strain gauge. The pressure measurements from the pressure gauge were also used to calibrate the specific voltages measured across the Wheatstone bridge to their corresponding pressure. The pressure was varied between $11 \mathrm{mmHg}$ to $14 \mathrm{mmHg}$. 


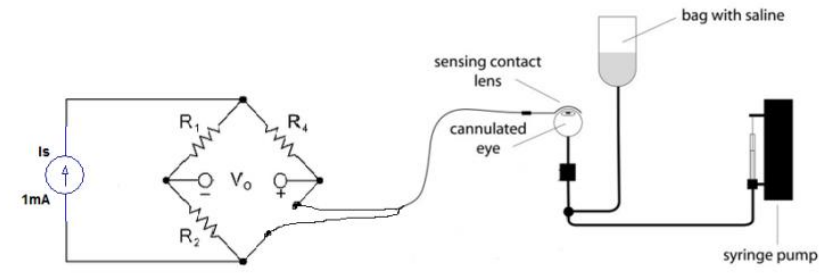

Figure 7.25 Final Testing Circuitry and Schematic

Aside from testing the gauge itself, the Wheatstone bridge was tested and demonstrated a linear curve as the active gauge resistance changes, as seen in Figure 7.26.

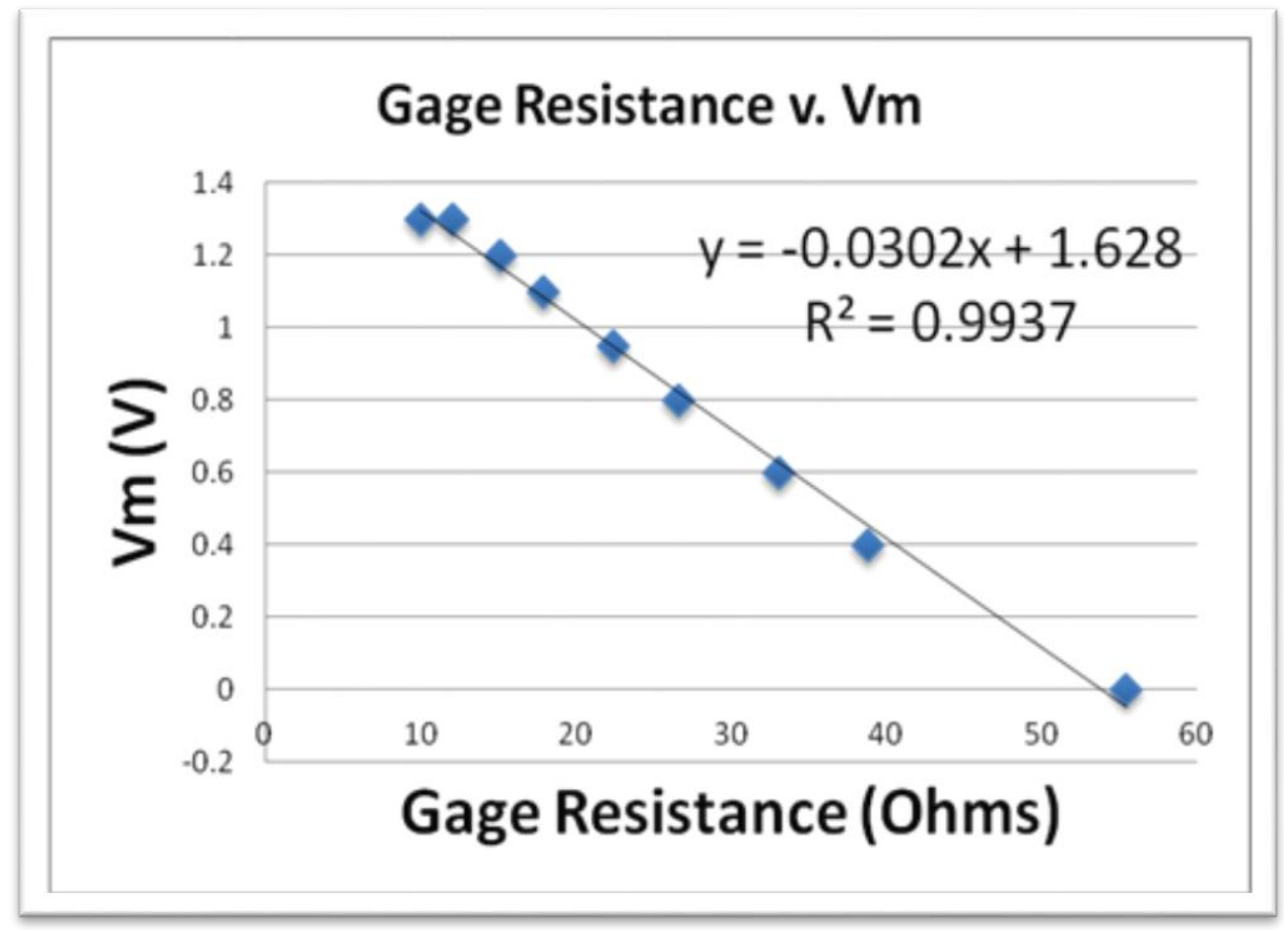

Figure 7.26 Wheatstone Bridge Measurement, Gage Resistance v. Vm This plot was attained by varying the resistance of the resistor in the Wheatstone bridge which was to act as the active gauge. This plot shows a linear relationship between $\mathrm{Vm}$ and $\mathrm{R}$ as the resistance increases. Characterizing the gauge factor of the strain gage was also attempted. The objective of characterization was to determine how big the resistance 
will change based on the strain induced on the device, where the gauge factor should be around 2 for most metals. The percentage of change can be determined by the following equation:

$$
\frac{\Delta R}{R}=G \varepsilon
$$

Where $\Delta R$ is the change in resistance, $R$ is the initial resistance without strain, $G$ is the gauge factor, and $\varepsilon$ is strain induced on the material. It was decided to use the relation of resistance to pressure to implement a Wheatstone Bridge Circuit that will make a relation from the change in pressure to the change in voltage. In result, the strain gauges worked and saw a change of 0.001 millivolts. Unfortunately, the measuring equipment we had were unable to read the range needed to determine the sensitivity of the circuit. Although the group I lead accomplished making a strain gage, we failed in providing enough data in determining whether this device can sense IOP changes. While the strain gage does have its resistance change based on how much strain it experiences, we do not have the measuring equipment to fully determine its capabilities. Thus, once we have access to better measuring devices, we can finalize the strain gauge's functionality. 


\section{Chapter 8}

\subsection{Conclusion}

The ability to screen print and micro-fabricate circuits on flexible and transparent substrates enables us to advance electronics into different markets such as the biomedical industry. In this thesis, I carried out the design and fabrication of components on a contact lens utilizing two different processing techniques. With the help of the screen printing from the Graphic Communications department, three types of antennas were fabricated that would provide communication at the 2.4GHz ISM band and inductive charging at $13.56 \mathrm{MHz}$. Out of the three antennas only two showed promise for implementation in the lens, the coil and IFA variation. The antennas were printed on $25 \mu \mathrm{m}$ PET substrate with a silver conductive ink by Dupont. The coil antenna produced a $S_{11}$ of $-4.45 \mathrm{~dB}$, a coil inductance of $268.2 \mathrm{nH}$ with a $\mathrm{Q}$-factor of 0.98 and a gain of $-13 \mathrm{~dB}$. The IFAbased design produced a $\mathrm{S}_{11}$ of $-9.582 \mathrm{~dB}$, a coil inductance of $273.4 \mathrm{nH}$ with a Q-factor of 0.269 and a gain of $-5.3 \mathrm{~dB}$. The display technology was also manufactured using the Graphic Communications department where a single element printed display was created using electrochromic ink. The ink in its discharged state is transparent and clear, but turns a transparent blue color when a potential of $3 \mathrm{~V}$ was applied. Microfabrication was also utilized to create the three antennas where the radiation patterns was determined as omnidirectional for all antennas in the anechoic chamber. Frequency response was also determined by S11 which showed better response to the oriented 
antennas. This is due to the microfabrication process being more accurate to printing and that sputtering metal has a higher conductivity than metal ink; having less resistance loss. The entire encapsulation process was done in the microfabrication lab. It was found that the printing process is good for prototyping individual components because quick changes to the design can be made in a short amount of time and at a low cost. However, microfabrication is a better process when developing the entire lens with embedded circuitry.

The battery chosen for our application was the CBC005 3.8V, $5 \mu \mathrm{Ah}$ thin film battery by Cymbet. This battery although rigid is very small and is able to provide a steady amount of power to the lens for an hour. The supercapacitor although successfully fabricated, still has concerns with potential leaking problems even with a separator. Also the supercapacitor has a different charge/discharge curve which is not as constant in potential versus time as a lithium ion battery. Unfortunately, all the components could not be assembled together due to unforeseen delays in the wirebonding process. The training for the wirebonding and items needed are well documented in this thesis and can be used as a reference.

Even though there were unforeseen challenges, we believe that the combination of biomedical ingenuity and novel functionality associated with our design sends a strong message to future groups of students looking to make active contact lenses. Our acceptance into the Engineering in Medicine and Biology Conference in Chicago, Illinois, first place award at the IDTech Printed Electronics Conference USA 2014 in Santa Clara, California, as well as the 
feedback from our sponsors, that our work has made a definitive impact on the cutting edge research of active contact lenses.

\subsection{Future Work}

At this point, we hope that future teams will take this work as a reference for the embedding of a functional platform into a contemporary contact lenses. There is still much work that needs to be done to ensure that our design meets the high standard set by the FDA for medical devices. Our heartfelt recommendation is that the work we performed in verifying the biomedical requirements of the device are improved upon, such that the device can one day be examined for in vivo and clinical study. Active contact lenses represent a milestone in communicating visual information to an increasingly diverse and technically competent population. It is our desire to see a device similar to ours one day contributing to the overall safety of the population monitoring users' vitals with wearable technology.

\subsubsection{Display}

The EC display implemented in this thesis was a single unit display made for the second prototype. Although the EC display was successfully designed and fabricated, there is room for improving the design. One way is to make an array of pixels so characters such as numbers or letters can be displayed on the lens. Another would be to introduce different color pixels such as yellow, green, or red. This can be done by changing the PEDOT:PSS layer with the following polymers in Table 6. 


\begin{tabular}{|l|l|r|}
\hline Polymer & Color Change & Applied Voltage (V) \\
\hline Polyaniline & Yellow to green & 2 \\
\hline Polythiophene & Red to blue & 1.5 \\
\hline Polypyrrole & Yellow to blue-black & N/A \\
\hline Polyisothianaphthene & Blue to light yellow &
\end{tabular}

Table 6 Characteristics of Electrochromic Cells Made from Conducting Polymers

\subsubsection{Manufacturing and Material}

Two different fabrication techniques (microfabrication and screen printing) were experimented in parallel, analyzed, and compared. During the printing process it was hard to get consistent $100 \mu \mathrm{m}$ feature sizes. Fortunately, the graphic communications department was able to acquire a new printing machine that does not require a pattern screen. So far the machine is able to print $100 \mu \mathrm{m}$ feature sizes and can be optimized to go below $50 \mu \mathrm{m}$. With a screen-less printer, alterations to the antenna can be done with no additional steps. All that is needed is to upload the design and substrate and it will print. As stated in the conclusion, printing is only good for prototyping and microfabrication is only good for manufacture a completed prototype. This is due to the material of the substrates where PDMS has a high contact angle making it impossible to print on and PET is not able to handle some of the chemical etchants used in the antenna process. The solution is using material from momentum that has a very low contact angle making it easy to print on. 
Since it is an altered form of PDMS, it should work for the microfabrication process as well.

Alternative processes were implemented and analyzed, one being microfluidics. The theory behind using microfluidics is that screen printing requires printing on a substrate that has good ink adhesion - which many biocompatible substrates are not. Using microfluidics, the antenna design could be formed into channels where ink can flow through. We tried this technique on a PDMS substrate and was able to flow in a channel that was $100 u m$ wide and 25 um thick. The viscosity of the ink poses a problem when trying to flow the ink through more narrow channels. This concern can be addressed two ways: 1 . We could alter the ink, such as adding alcohols, to lower it viscosity or 2. Add more vias for the microfluidics nozzle such that more force could be applied to push the ink through. Future projects could also employ different types of ink other than silver such as gold, copper, or Henkel ECl-5001.

\subsubsection{Electrical}

The amplification stage as well as the voltage regulator could be improved on. The design of the common source amplifier power consumption can be reduced significantly if subthreshold techniques were used. One subthreshold design. Lee et al. [18] utilizes a typology that is similar to the LNA topology used in the custom IC. The entire receiving block can be implemented with subthreshold design which would decrease power consumption from mWatts to $\mu$ Watts. Circuit designs that bias MOSFETS in the subthreshold region require tight process 
tolerances as well as accurate biasing. However, if ultra-low power design could be achieved, then the battery can power the system for an entire day if not more. The voltage regulator needs to be more stable by either improving the existing topology or experimenting with other topologies.

\subsubsection{Antenna Design}

Initially for this project, conductive ink, Henkel ECI-5001, was ordered as the material to make the antenna. Unfortunately, the ink did not arrive until a year later and an alternative material (gold and silver ink) were used. Having a clear conductive ink allows us to alter the antenna design such that its features can run across the user's vision. For example, we would not have to open up the ground plane in the IFA design in Chapter 3 which will increase the efficiency of the antenna. The transparent ink may also allow future coil designs to incorporate more turns to achieve a higher inductance. Another option would be to layer the ground plane, antenna, and coil on separate planes.

\subsubsection{Applications}

A prototype for charging at $13.56 \mathrm{MHz}$ has been developed to test charging applications as well as a UV sensor to drive the lens at $2.4 \mathrm{GHz}$ to test the display response on-lens system. As stated prior there are limitless applications for the active lens. The following list are some applications that can be researched.

- Having an app developed to interface the lens with a phone. 
- Transmitting data from the lens to a device.

- Communication from one lens to another lens.

- Drug delivery system using embedded micro-wells. 


\section{REFERENCES}

[1] S. Iguchi, H. Kudo, T. Saito, M. Ogawa, H. Saito, K. Otsuka, A. Funakubo, and K. Mitsubayashi, "A flexible and wearable biosensor for tear glucose measurement.," Biomed. Microdevices, vol. 9, no. 4, pp. 603-9, Aug. 2007.

[2] H. Yao, A. J. Shum, M. Cowan, I. Lähdesmäki, and B. a Parviz, "A contact lens with embedded sensor for monitoring tear glucose level.," Biosens. Bioelectron., vol. 26, no. 7, pp. 3290-6, Mar. 2011.

[3] J. Pandey, Y. Liao, and A. Lingley, "A fully integrated RF-powered contact lens with a single element display," ... Circuits Syst. ..., vol. 4, no. 6, pp. 454-461, 2010.

[4] Errol Leon, Benny Ng, and Paul Hecklar, "Clear Circuits for Contact Lens Applications," 2013.

[5] Errol Leon, Alex Do, Paul Hecklar, and Phil Azar: 'Clear Active Contact Lens (CACL)'. 2014.

[6] Benny Ng, 'Embedded Power Active Contact Lens,' 2015.

[7] Y. Lee: Antenna Circuit Design for RFID Applications. 2003. URL: http://ww1.microchip.com/downloads/en/AppNotes/00710c.pdf (visited on 01/13/2014).

[8] W.L. Stutzman and G. A. Thiele: Antenna Theory and Design. Hoboken, N.J.: John Wiley Sons, Inc, 2012.

[9] P.J. Bevelacqua: Inverted-F Antenna (IFA). URL: http://www.antenna-theory.com/antennas/aperture/ifa.php (visited on 04/06/2014).

[10] Liu, Chenguang, Zhenning Yu, David Neff, Aruna Zhamu, and Bor Z. Jang. "Graphene-Based Supercapacitor with an Ultrahigh Energy Density." Nano Letters 10.12 (2010): 4863-868. Print.

[11] "Graphene." Wikipedia. Wikimedia Foundation, 25 Jan. 2014. Web. 27 Jan. 2014.

[12] S.J. Wang, Y. Geng, Q. Zheng and J.k. Kim, "Fabrication of highly conducting and transparent graphene films," Carbon, 48, 18151823 (2010). 
[13] Grace Lao, "Nematic Order Drives Macroscopic patterns of Graphene Oxide in Drying Drops," American Chemistry Society Langmuir 30 (2014): 14631-14637.

[14] Jun Kawahara, Peter Andersson Ersman, Isak Engquist, Magnus Berggren, "Improving the color switch contrast in PEDOT:PSSbased electrochromic displays," Organic Electronics, 13, 469-474 (2012).

[15] Seung Cho, "Nanotube based Ultrafast Electrochromic Display," Advance Materials, 17, No 2, 171-175 (2005).

[16] Hans Meyer, "Soft Lithography Slides," Department of Mechanical Engineering, Feb. 27, 2014.

[17] Leonard Sax (2010). "Polyethylene Terephthalate May Yield Endocrine Disruptors". Environmental Health Perspectives 118 (4): 445-8.

[18] Naoki Sadayori and Yuji Hotta "Polycarbodiimide having high index of refraction and production method thereof" US patent 2004/0158021 A1 (2004).

[19] McCrea, Keith R., Yuan Tian, and Robert S. Ward. "Silicone hydrogels for tissue adhesives and tissue dressing applications." U.S. Patent Application 12/745,509.

[20] Gokul, K. C., D. B. Gurung, and P. R. Adhikary. "Effect of Blood Perfusion and Metabolism in Temperature Distribution in Human Eye." Advances in Applied Mathematical Biosciences 4.1 (2013): 13-23.

[21] Cvetkovic, Mario, Dragan Poljak, and Andres Peratta. "Thermal modelling of the human eye exposed to laser radiation." Software, Telecommunications and Computer Networks, 2008. SoftCOM 2008. 16th International Conference on. IEEE, 2008.

[22] WORLD HEALTH ORGANIZATION: INTERSUN - The global UV Project: A guide and compendium. 2003.

URL:http://www.who.int/uv/publications/en/Intersunguide.pdf?ua=1

[23] A.M. Madni and L.A. Wan, "Micorelectromechanical Systems (MEMS): an overview of current state-of-the-art," in Aerospace Conference, pp. 421-427, March 21-28, 1998. 
[24] M. Leonardi, E. M. Pitchon, A. Bertsch, P. Renaud and A. Mermoud, "Wireless contact lens sensor for intraocular pressure monitoring: assessment on enucleated pig eyes," Acta Ophthalmologica, vol. 87, pp. 433-437, 2009.

[25] T. S. Prata, et al. "Posture-induced Intraocular Pressure Changes: Considerations Regarding Body Position in Glaucoma Patients." Survey of Ophthalmology. vol. 55, num. 5, Sept. 2010.

[26] K.K. O'Brien, A.W.Y. Chock, C.A. Opere, "An overview of glaucoma management for pharmacists" http://www.uspharmacist.com/continuing_education/ceviewtest/les sonid/106698, April 2010.

[27] S.C. Sacca, C. Cartiglia, A. Izzotti, "Glaucoma: an overview." Handbook of Nutrition, Diet and the Eye. pp. 29-40, Elsevier, 2014.

[28] Gupta Eye Centre "Glaucoma," http://guptaeyecentre.com/glaucoma.htm, October 2011.

[29] E. Yung, V. Trubnik, and L.J. Katz, "An overview of home tonometry and telemetry for intraocular pressure monitoring in humans," Graefes Arch Clin Exp Ophthalmol, pp. 1-10. May 2014.

[30] A.K.C. Lam and W. A. Douthwaite, "The effect of an artificially elevated intraocular pressure on the central corneal curvature," Ophthal. Physiol. Opt, vol. 17, no. 1, pp. 18-24, 1997.

[31] M. Leonardi et al. "A Soft Contact Lens with a MEMs Strain Gage Embedded for Intraocular Pressure Monitoring." Centre Medical Univeritaire. 2003.

[32] B. Jaffe. Piezoelectric ceramics. Vol. 3. Elsevier, 2012.

[33] H. W. CHENG, B. M. JENG, H. Y. HUANG, J. C. CHIOU, and C. H. LUO: 'The Rectenna Design on Contact Lens for Wireless Powering of the Active Intraocular Pressure Monitoring System'. InIEEE EMBS. (Osaka). 2013.

[34] CYMBET CORPORATION: EnerChip Standards Compliance and Use Procedures. 2012. URL: http://www.cymbet.com/pdfs/PI-7204.pdf.

[35] "Fatal Occupational Injuries in 2012 - Chart Package." Occupational Health and Safety Administration. U.S. Department of Labor, 2012. Web. 20 Jan. 2014. 
[36] Lingley, Andrew R., et al. "A single-pixel wireless contact lens display." Journal of Micromechanics and Microengineering 21.12 (2011): 125014.

[37] B. Parviz, "For your eye only," Spectrum, IEEE, no. september 2009, pp. 36-41, 2009.

[38] H. Yao, A. J. Shum, M. Cowan, I. Lähdesmäki, and B. a Parviz, "A contact lens with embedded sensor for monitoring tear glucose level.," Biosens. Bioelectron., vol. 26, no. 7, pp. 3290-6, Mar. 2011.

[39] A. Kagie, D. K. Bishop, J. Burdick, J. T. La Belle, R. Dymond, R. Felder, and J. Wang, "Flexible Rolled Thick-Film Miniaturized Flow-Cell for Minimally Invasive Amperometric Sensing," Electroanalysis, vol. 20, no. 14, pp. 1610-1614, Jul. 2008.

[40] S. Iguchi, H. Kudo, T. Saito, M. Ogawa, H. Saito, K. Otsuka, A. Funakubo, and K. Mitsubayashi, "A flexible and wearable biosensor for tear glucose measurement.," Biomed. Microdevices, vol. 9, no. 4, pp. 603-9, Aug. 2007.

[41] P. C. Nicolson and J. Vogt, "Soft contact lens polymers: an evolution." Biomaterials, vol. 22, no. 24, pp. 3273-83, Dec. 2001.

[42] "Medical Devices." Radio Frequency Wireless Technology in. FDA, 13 Aug. 2013. Web. 26 Jan. 2014.

[43] L. B. Kish, "End of Moore's Law: Thermal (Noise) Death of Integration in Micro and Nano Electronics," Phys. Lett. A. 305144 (2002).

[44] R.M. Ford and C.S. Coulston, Design for Electrical and Computer Engineers, McGraw-Hill, 2007.

[45] El-Kady, Maher. "Laser Scribing of High-Performance and Flexible Graphene-Based Electrochemical Capacitors - Supporting Online Material." Laser Scribing of High-Performance and Flexible Graphene-Based Electrochemical Capacitors - Supporting Online Material. Science Magazine, 16 Mar. 2012. Web. 3 Nov. 2013.

[46] Seitz, Helmut, et al. "Biocompatibility of polyethylene terephthalate (Trevira $<$ sup $>\AA</$ sup $>$ hochfest) augmentation device in repair of the anterior cruciate ligament." Biomaterials 19.1 (1998): 189-196.

[47] Seitz, H., et al. "Biocompatibility of polyethylene terephthalate-PET--(Trevira strong)--an in vivo study of the sheep knee." 
Biomedizinische Technik. Biomedical engineering 41.6 (1996): 178.

[48] Merkel, T. C., et al. "Gas sorption, diffusion, and permeation in poly (dimethylsiloxane)." Journal of Polymer Science Part B: Polymer Physics 38.3 (2000): 415-434.

[49] Weinbaum, S., and L. M. Jiji. "A new simplified bioheat equation for the effect of blood flow on local average tissue temperature." Journal of biomechanical engineering 107.2 (1985): 131-139.

[50] "Flat Spiral Coil Design Calculator." Flat Spiral Coil Design Calculator. Circuits, 9 Dec. 2010. Web. 27 Jan. 2014.

[51] Liu, Chenguang, Zhenning Yu, David Neff, Aruna Zhamu, and Bor Z. Jang. "Graphene-Based Supercapacitor with an Ultrahigh Energy Density." Nano Letters 10.12 (2010): 4863-868. Print.

[52] Richard Savage, "PMOS Process Traveler Booklet Version 7.8," 19 Dec. 2013. 


\section{APPENDICES}

\section{Appendix A: Datasheets}

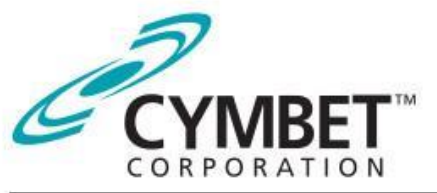

\section{EnerChip ${ }^{\mathrm{TM}}$ CBC050}

\section{Rechargeable Solid State Energy Storage: $50 \mu \mathrm{Ah}, 3.8 \mathrm{~V}$}

\author{
Features \\ - All Solid State Construction \\ - SMT Package and Process \\ - Lead-Free Reflow Tolerant \\ - Thousands of Recharge Cycles \\ - Low Self-Discharge \\ - Eco-Friendly, RoHS Compliant
}

\section{Electrical Properties}

$\begin{array}{ll}\text { Output voltage: } & 3.8 \mathrm{~V} \\ \text { Capacity (typical): } & 50 \mu \mathrm{Ah} \\ \text { Charging source: } & 4.00 \mathrm{~V} \text { to } 4.15 \mathrm{~V} \\ \text { Recharge time to } 80 \%: & 20 \text { minutes } \\ \text { Charge/Discharge cycles: } & >5000 \text { to } 10 \% \text { DOD }\end{array}$

Physical Properties

Package size:

Operating temperature:

Storage temperature:

$8 \mathrm{~mm} \times 8 \mathrm{~mm}$ $-20^{\circ} \mathrm{C}$ to $70^{\circ} \mathrm{C}$ $-40^{\circ} \mathrm{C}$ to $125^{\circ} \mathrm{C}$

\section{Applications}

- Standby supply for non-volatile SRAM, real-time clocks, controllers, supply supervisors, and other system-critical components.

- Wireless sensors and RFID tags and other powered, low duty cycle applications.

- Localized power source to keep microcontrollers and other devices alert in standby mode.

- Power bridging to provide backup power to system during exchange of main batteries.

- Energy Harvesting by coupling the EnerChip with energy transducers such as solar panels.

- Embedded Energy where bare die can be embedded into modules or co-packaged with other ICs.

\begin{tabular}{|c|c|}
\hline Pin Number(s) & Description \\
\hline 1 & V+ \\
\hline 4 & V- \\
\hline 2,3 & NIC \\
\hline $5-16$ & NIC \\
\hline \multicolumn{2}{|c|}{ Note: NIC = No Internal Connection } \\
\hline
\end{tabular}

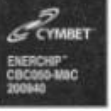

$8 \mathrm{~mm} \times 8 \mathrm{~mm}$ QFN SMT Package

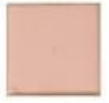

$5.7 \mathrm{~mm} \times 6.1 \mathrm{~mm}$ Bare Die
The EnerChip ${ }^{\mathrm{TM}} \mathrm{CBC050}$ is a surface-mount, solid state, rechargeable energy storage device rated for $50 \mu \mathrm{Ah}$ at $3.8 \mathrm{~V}$. It is ideal as a localized, onboard power source for SRAMs, real-time clocks and microcontrollers which require standby power to retain time or data. It is also suitable for RFID tags, smart sensors, and remote applications which require a miniature, low-cost, and rugged power source. For many applications, the $\mathrm{CBCO50}$ is a superior alternative to coin cell batteries and supercapacitors.

Because of their solid state design, EnerChip ${ }^{\mathrm{TM}}$ storage devices are able to withstand solder reflow temperatures and can be processed in highvolume manufacturing lines similar to conventional semiconductor devices. There are no harmful gases, liquids or special handling procedures, in contrast to traditional rechargeable batteries.

The EnerChip recharge is fast and simple, with a direct connection to a $4.1 \mathrm{~V}$ voltage source and no current limiting components. Recharge time is 20 minutes to $80 \%$ capacity. Robust design offers thousands of charge/discharge cycles. The CBCO50 is packaged in an $8 \mathrm{~mm} \times 8 \mathrm{~mm}$ quad flat package. It is available in reels for use with automatic insertion equipment.

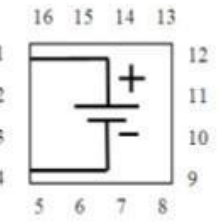

CBC050 Schematio - Top View 


\section{EnerChip ${ }^{\mathrm{TM}}$ CBC050 Solid State Energy Storage}

\section{Operating Characteristics}

\begin{tabular}{|c|c|c|c|c|c|c|}
\hline \multicolumn{2}{|c|}{ Parameter } & Condition & Min & Typical & Max & Units \\
\hline \multicolumn{2}{|l|}{ Discharge Cutoff Voltage } & $25^{\circ} \mathrm{C}$ & $3.0^{(1)}$ & - & - & V \\
\hline \multicolumn{2}{|l|}{ Charge Voltage } & $25^{\circ} \mathrm{C}$ & $4.0^{23}$ & 4.1 & 4.3 & V \\
\hline \multicolumn{2}{|l|}{ Pulse Discharge Current } & $25^{\circ} \mathrm{C}$ & $300^{3 i}$ & - & - & $\mu \mathrm{A}$ \\
\hline \multirow{2}{*}{\multicolumn{2}{|c|}{ Cell Resistance $\left(25^{\circ} \mathrm{C}\right)$}} & Charge cycle 2 & - & 750 & 2000 & \multirow{2}{*}{$\Omega$} \\
\hline & & Charge cycle 1000 & - & 4200 & 7000 & \\
\hline \multirow{2}{*}{\multicolumn{2}{|c|}{ Self-Discharge (5yr average; $25^{\circ} \mathrm{C}$ ) }} & Non-recoverable & - & 2.5 & - & $\%$ per year \\
\hline & & Recoverable & - & $1.5^{(4)}$ & - & $\%$ per year \\
\hline \multicolumn{2}{|l|}{ Operating Temperature } & - & -20 & 25 & +70 & ${ }^{\circ} \mathrm{C}$ \\
\hline \multicolumn{2}{|l|}{ Storage Temperature } & - & -40 & - & $125^{\text {(5) }}$ & ${ }^{\circ} \mathrm{C}$ \\
\hline \multirow{4}{*}{$\begin{array}{l}\text { Recharge Cycles } \\
\text { (to } 80 \% \text { of rated } \\
\text { capacity; } 4.1 \mathrm{~V} \text { charge } \\
\text { voltage) }\end{array}$} & \multirow[t]{2}{*}{$25^{\circ} \mathrm{C}$} & $10 \%$ depth-of-discharge & 5000 & - & - & cycles \\
\hline & & $50 \%$ depth-of discharge & 1000 & - & - & cycles \\
\hline & \multirow[t]{2}{*}{$40^{\circ} \mathrm{C}$} & $10 \%$ depth-of-discharge & 2500 & - & - & cycles \\
\hline & & $50 \%$ depth-of-discharge & 500 & - & - & cycles \\
\hline \multirow{2}{*}{\multicolumn{2}{|c|}{$\begin{array}{l}\text { Recharge Time (to } 80 \% \text { of rated capacity; } \\
4.1 \mathrm{~V} \text { charge voltage) }\end{array}$}} & Charge cycle 2 & - & 20 & 35 & \multirow{2}{*}{ minutes } \\
\hline & & Charge cycle 1000 & - & 60 & 95 & \\
\hline \multicolumn{2}{|l|}{ Capacity } & $100 \mu \mathrm{A}$ discharge $; 25^{\circ} \mathrm{C}$ & 50 & - & - & $\mu A h$ \\
\hline
\end{tabular}

(1) Failure to cutoff the discharge voltage at $3.0 \mathrm{~V}$ will result in EnerChip performance degradation.

(2) Charging at $4.0 \mathrm{~V}$ will charge the cell to approximately $70 \%$ of its rated capacity.

(3) Typical pulse duration $=20$ milliseconds.

(4) First month recoverable self-discharge is $5 \%$ average.

(5) Storage temperature is for uncharged EnerChip.

Note: All specifications contained within this document are subject to change without notice

\section{EnerChip Discharge Characteristics}

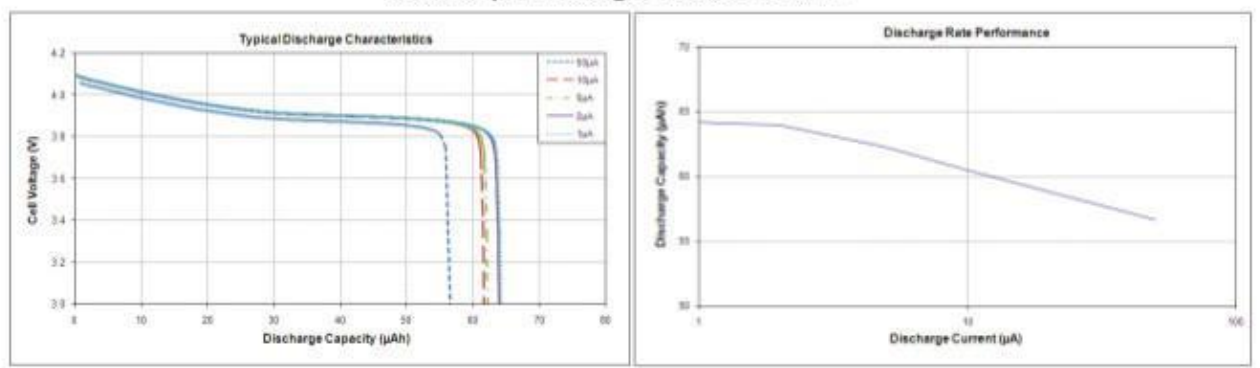

\section{Ordering Information}

\begin{tabular}{|c|c|c|}
\hline EnerChip Part Number & Description & Notes \\
\hline CBC050-M8C & 50 4 Ah in 16-pin M8 QFN Package & tube \\
\hline CBC050-M8C-TR1 & $50 \mu \mathrm{Ah}$ in 16-pin M8 QFN Package & tape \& reel 1000 pcs \\
\hline CBC050-M8C-TR5 & 50 $\mu \mathrm{Ah}$ in 16-pin M8 QFN Package & tape \& reel 5000 pcs \\
\hline CBC050-M8C-WP & 50 $\mu$ Ah in 16-pin M8 QFN Package & waffle pack \\
\hline CBC050-BDC-WP & 50 $\mu$ Ah Bare Die & Contact Cymbet \\
\hline CBC050-BUC-WP & 50uAh Bumped Bare Die & Contact Cymbet \\
\hline
\end{tabular}




\section{Package Dimensions - 16-pin QFN (package code M8)}

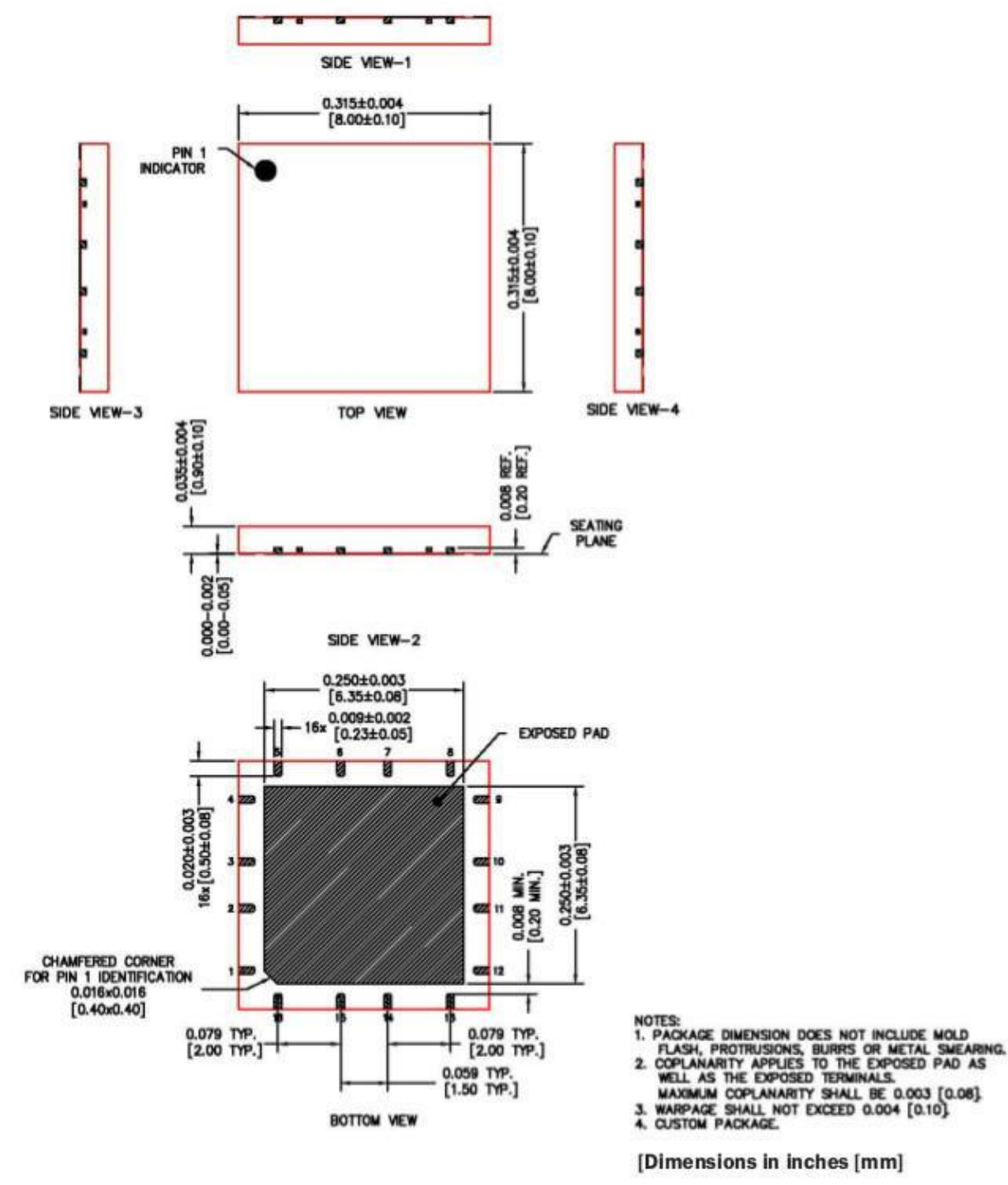


EnerChip ${ }^{\mathrm{TM}}$ CBC050 Solid State Energy Storage

\section{Printed Circuit Board (PCB) Layout Guidelines and Recommendations}

Electrical resistance of solder flux residue on PCBs can be low enough to partially or fully discharge the backup energy cell and in some cases can be comparable to the load typically imposed on the cell when delivering power to an integrated circuit in low power mode. Therefore, solder flux must be thoroughly washed from the board following soldering. The PCB layout can make this problem worse if the cell's positive and negative terminals are routed near each other and under the package, where it is difficult to wash the flux residue away.

To avoid this situation, make sure positive and negative traces are routed outside of the package footprint to ensure that flux residue will not cause a discharge path between the positive and negative pads. Similarly, a leakage current path can exist from the package lead solder pads to the exposed die pad on the underside of the package as well as any solder pad on the PCB that would be connected to that exposed die pad during the reflow solder process. Therefore, it is strongly recommended that the PCB layout not include a solder pad in the region where the exposed die pad of the package will land. It is sufficient to place PCB solder pads only where the package leads will be. That region of the PCB where the exposed die pad will land must not have any solder pads, traces, or vias.

When placing a silk screen on the PCB around the perimeter of the package, place the silk screen outside of the package and all metal pads. Failure to observe this precaution can result in package cracking during solder reflow due to the silk screen material interfering with the solder solidification process during cooling.

A recommended CBC050 PCB layout is shown in Figure 1 below. Notice that there should not be a center pad on the PCB to mate with the exposed die pad on the CBC050 package. Again, this is to reduce the possible number and severity of leakage paths between the EnerChip terminals.

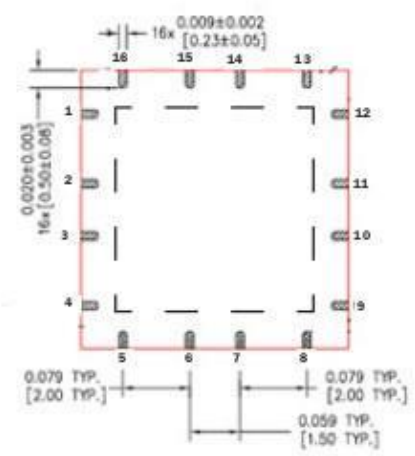

Dimensions in inches [mm]

Figure 1: Recommended PCB layout for the CBCO50 package. Do not route signal traces under the EnerChip as they could become shorted to the die pad (as shown by the dotted lines) on the package underside.

\section{Soldering, Rework, and Electrical Test}

Refer to the Cymbet User Manual for soldering, rework, and replacement of the EnerChip on printed circuit boards, and for instructions on in-circuit electrical testing of the EnerChip.

\section{Disclaimer of Warranties; As is}

The information provided in this data sheet is provided "As is" and Cymbet Corporation disclaims all representations or warranties of any kind, express or implied, relating to this data sheet and the Cymbet EnerChip product described herein, including without limitation, the implied warranties of merchantability, fitness for a particular purpose, non-infringement, title, or any warranties arising out of course of dealing, course of performance, or usage of trade. Cymbet EnerChip products are not approved for use in life critical applications. Users shall confirm suitability of the Cymbet EnerChip product in any products or applications in which the Cymbet EnerChip product is adopted for use and are solely responsible for all legal, regulatory, and safety-related requirements conceming their products and applications and any use of the Cymbet EnerChip product described herein in any such product or applications.

Cymbet, the Cymbet Logo and EnerChip are trademarks of Cymbet Corporation. All Rights Reserved 


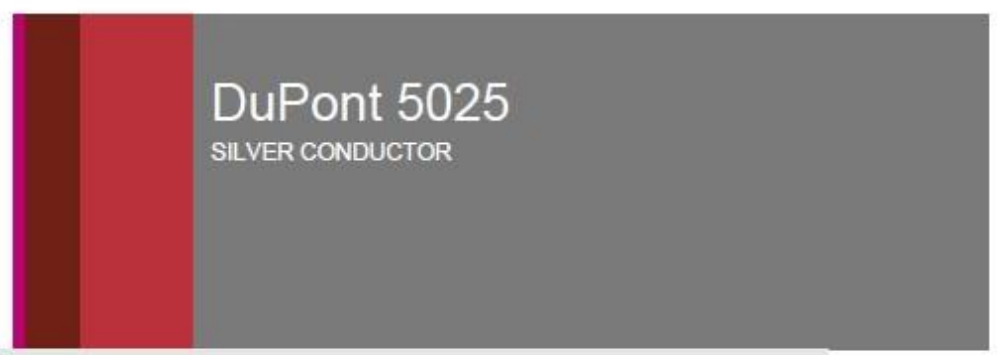

Technical Data Sheet

\section{Product Description}

DuPont 5025 silver conductor is used to fabricate low voltage circuitry, especially on flexible substrates. It can be used with manual, semiautomatic, and reel-to-reel equipment.

\section{Product Benefits}

- Best general purpose Ag for higher temperature operations

\section{Processing}

- Screen Printing Equipment Reel-to-reel, Semiautomatic, manual

\section{- Substrates}

Polyester, polyimide, paper, epoxy glass

- Ink Residence Time on Screen $>2$ hours

- Screen Types

Stainless steel, Polyester

- Typical Cure Conditions Box Oven: $120^{\circ} \mathrm{C}$ for $5-6$ minutes Reel-to-reel: $140^{\circ} \mathrm{C}$ for 1 minute

- Typical Circuit Line Thickness Printed with 325-mesh stainless steel screen 12 - 15 microns

- Clean-up Solvent

Ethylene diacetate or methyl propasol acetate

\section{Table 1}

Typical Physical Properties on 5-mil Polyester Film

\begin{tabular}{|c|c|}
\hline Test & Properties \\
\hline Sheet Resistivity $(\mathrm{m} \Omega / \mathrm{sq} / \mathrm{mil})^{*}$ & $12-15$ \\
\hline 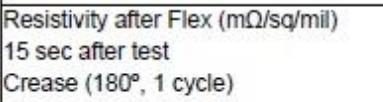 & $\leq 50$ \\
\hline \begin{tabular}{|l} 
Adhesion/Tape Pull \\
(3M Scotch Tape \#600)
\end{tabular} & No Ag Transfer \\
\hline \begin{tabular}{|l|} 
Abrasion Resistance, Pencil \\
Hardness (ASTM D3363-74) [H]
\end{tabular} & $\geq 1$ \\
\hline Operating Use Temperature $\left({ }^{\circ} \mathrm{C}\right)$ & $\leq 110$ \\
\hline Solderability & Not Recommended \\
\hline $\begin{array}{l}\text { Change is Physical Properties after } \\
\text { Environment Test * }\end{array}$ & Insignificant \\
\hline $\begin{array}{l}\text { Change is Physical Properties after } \\
\text { Environment Test }{ }^{*}(\%)\end{array}$ & $<10$ \\
\hline \multicolumn{2}{|c|}{ 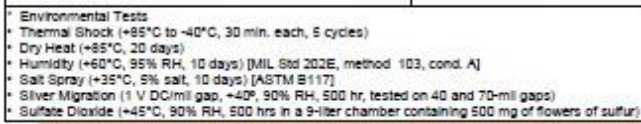 } \\
\hline $\begin{array}{l}\text { Table } 1 \text { \& } 2 \text { show anticipated typical p } \\
5025 \text { based on specific controlled exper } \\
\text { intended to represent the product speci } \\
\text { available upon request. }\end{array}$ & $\begin{array}{l}\text { properties for DuPon } \\
\text { in our labs and are no } \\
\text { is, detalls of which are }\end{array}$ \\
\hline
\end{tabular}




Table 2
Composition Properties
\begin{tabular}{|l|c|}
\hline \multicolumn{1}{|c|}{ Test } & Properties \\
\hline $\begin{array}{l}\text { Viscosity (Pa.S) } \\
\left.\text { Brookfield 0.5RVT, 5rpm \#14, 25 }{ }^{\circ} \mathrm{C}\right]\end{array}$ & $20-30$ \\
\hline Solids $\left(150^{\circ} \mathrm{C}\right)[\%]$ & $68-72$ \\
\hline $\begin{array}{l}\text { Coverage }\left(\mathrm{cm}^{2} / \mathrm{g}\right) \\
\text { Dependent on print thickness] }\end{array}$ & $230-320$ \\
\hline Thinner & DuPont 8210 \\
\hline
\end{tabular}

Dry

Dry and cure in a well ventilated oven or conveyor dryer where the exhaust meets environmental regulations.

\section{Storage and Shelf Life}

Containers should be stored, tightly sealed, in a clean, stable environment at room temperature $\left(<25^{\circ} \mathrm{C}\right)$. Shelf life of material in unopened containers is six months from date of shipment. Some settling of solids may occur and compositions should be thoroughly mixed prior to use.

Safety and Handling

For Safety and Handling information pertaining to this product, read the Material Safety Data Sheet (MSDS).

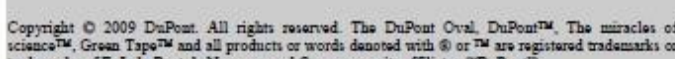

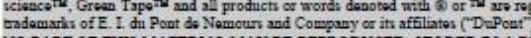

NO PART OF THIS MATERIAL MAY BE REPRODUCED, STORED DV A RETRIEVAI PHOTOCOPYNG RECORN OR BY ANY MEANS ELECTRONIC, PRTO WRTTIEN PERMISSTON OF DUPONT

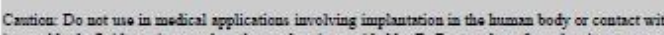

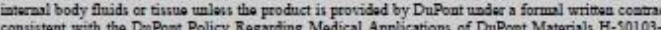

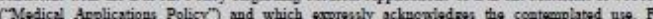

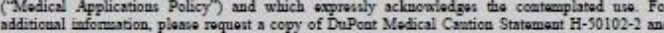
the DuPout Modical Applications Policy.

The informustion provided barein is offared for the prodact meer's considerntion rad examinasion

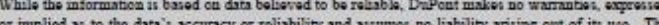

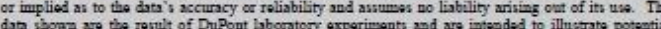

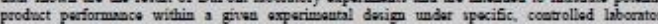

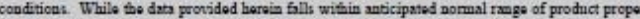

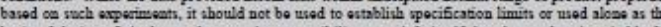

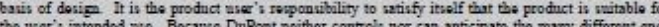

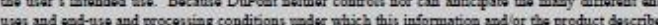

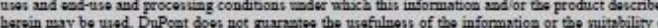

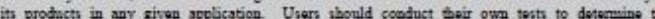
appropriatesess of the groduct for thair particulyr purpose.

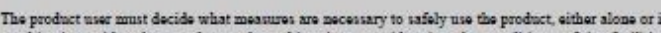
combination with otber products, slso thing into consideration the conditions of its faciletio

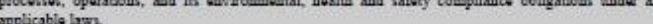
applicable lawn

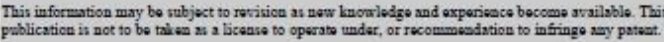

(1010OND.

The miracles of science
For more intormation on DuPont 5025 or other DuPant Microcircult Materlals products, please contact your iocal representative: Americas

DuPont Microcircult Materia:

14 T.W. Alexander Drive

Research Trlangle Park, NC 27709

Tel: $800-284-3382$

Eurone

Du Pont (U.K.) Limited

Coldharbour Lane

Bristol BS16 1 QD

U.K.

Tel.: 44-117-931-3191

Ast3

DuPont Kabushikl Kalsha

Sanno Park Tower, 11-1

Nagata-cho 2-chome

Chiyoda-ku, Tokyo 100-511

Japan

Tel: 81-3-5521-6650

Dupont Tawan Lto

45, Hising-Pont Road,

Taoyuan, Tawan 330

Tel: $886-3-377-3616$

Dupont China Holding Co. Lid

Bidg 11, 399 Keyuan Rd., Znangl HI-Tech Park, Pudong New District, Snanghal 201203, China

Tel: : 86-21-6386-6366 ext 2202

DuPont Korea inc.

3 5 th Floor, Asla tower $\$ 726$,

Yeoksam-dong, Gangnam-gu

Seoul 135-719, Korea

Tel.: 82-10-6385-5399

E. L. DuPont indla Private LImited 7th Floor, Tower C, DLF Cyper Greens, Sector-25A, DLF City, Phase-lil, Gurgaon 122002 Haryana, India Tel: 91-124-4091818

Du Pont Company (Singapore) Pte Lto

1 Harbourfront Place, \#11-01

Harbourfrong Tower One,

Singapore 098633

Tel.: 65-6586-3022

nttp:IImem.dupont.com

MCM5025 (08/2014) 


\section{Material Safety Data Sheet Propylene carbonate MSDS}

\begin{tabular}{|c|c|}
\hline \multicolumn{2}{|c|}{ Section 1: Chemical Product and Company Identification } \\
\hline Product Name: Propylene carbonate & Contact Information: \\
\hline $\begin{array}{l}\text { Catalog Codes: SLP } 3979 \\
\text { CAS\#: } 108-32-7\end{array}$ & $\begin{array}{l}\text { Sciencelab.com, Inc. } \\
14025 \text { Smith Rd. } \\
\text { Houston, Texas } 77396\end{array}$ \\
\hline RTECS: FF9850000 & $\begin{array}{l}\text { US Sales: } 1-800-901-7247 \\
\text { International Sales: } 1-281-441-4400\end{array}$ \\
\hline TSCA: TSCA 8(b) inventory: Propylene carbonate & Order Online: ScienceLab.com \\
\hline $\begin{array}{l}\text { Cl\#: Not available. } \\
\text { Synonym: 4-Methyl-2-oxo-1,3-dioxolane }\end{array}$ & $\begin{array}{l}\text { CHEMTREC (24HR Emergency Telephone), call: } \\
1-800-424-9300\end{array}$ \\
\hline Chemical Formula: $\mathrm{C} 4 \mathrm{H} 6 \mathrm{O} 3$ & International CHEMTREC, call: 1-703-527-3887 \\
\hline & For non-emergency assistance, call: $1-281-441-4400$ \\
\hline
\end{tabular}

\begin{tabular}{|c|c|c|}
\hline \multicolumn{3}{|c|}{ Section 2: Composition and Information on Ingredients } \\
\hline \multicolumn{3}{|l|}{ Composition: } \\
\hline Name & CAS \# & \% by Weight \\
\hline Propylene carbonate & $108-32-7$ & 100 \\
\hline
\end{tabular}

\section{Section 3: Hazards Identification}

Potential Acute Health Effects: Hazardous in case of skin contact (irritant), of eye contact (irritant), of ingestion, of inhalation. Potential Chronic Health Effects:

CARCINOGENIC EFFECTS: Not available. MUTAGENIC EFFECTS: Not available. TERATOGENIC EFFECTS: Not available. DEVELOPMENTAL TOXICITY: Not available. Repeated or prolonged exposure is not known to aggravate medical condition.

\section{Section 4: First Aid Measures}

Eye Contact:

Check for and remove any contact lenses. Immediately flush eyes with running water for at least 15 minutes, keeping eyelids open. Cold water may be used. Do not use an eye ointment. Seek medical attention.

Skin Contact: 
After contact with skin, wash immediately with plenty of water. Gently and thoroughly wash the contaminated skin with running water and non-abrasive soap. Be particularly careful to clean folds, crevices, creases and groin. Cold water may be used. Cover the irritated skin with an emollient. If irritation persists, seek medical attention. Wash contaminated clothing before reusing.

Serious Skin Contact:

Wash with a disinfectant soap and cover the contaminated skin with an anti-bacterial cream. Seek medical attention.

Inhalation: Allow the victim to rest in a well ventilated area. Seek immediate medical attention.

Serious Inhalation: Not available.

Ingestion:

Do not induce vomiting. Loosen tight clothing such as a collar, tie, belt or waistband. If the victim is not breathing, perform mouth-to-mouth resuscitation. Seek immediate medical attention.

Serious Ingestion: Not avalable.

\section{Section 5: Fire and Explosion Data}

Flammability of the Product: May be combustible at high temperature.

Auto-Ignition Temperature: $510^{\circ} \mathrm{C}\left(850^{\circ} \mathrm{F}\right)$

Flash Points: CLOSED CUP: $132^{\circ} \mathrm{C}\left(269.6^{\circ} \mathrm{F}\right)$. OPEN CUP: $135^{\circ} \mathrm{C}\left(275^{\circ} \mathrm{F}\right)$.

Flammable Limits: Not available.

Products of Combustion: These products are carbon oxides ( $\mathrm{CO}, \mathrm{CO} 2)$

Fire Hazards in Presence of Various Substances: Not available.

Explosion Hazards in Presence of Various Substances:

Risks of explosion of the product in presence of mechanical impact: Not available. Risks of explosion of the product in presence of static discharge: Not avalable.

Fire Fighting Media and Instructions:

SMALL FIRE: Use DRY chemical powder. LARGE FIRE: Use water spray, fog or foam. Do not use water jet.

Special Remarks on Fire Hazards: Not available.

Special Remarks on Explosion Hazards: Not available.

\section{Section 6: Accidental Release Measures}

Small Spill:

Dilute with water and mop up, or absorb with an inert dry material and place in an appropriate waste disposal container. Finish cleaning by spreading water on the contaminated surface and dispose of according to local and regional authority requirements.

Large Spill:

Absorb with an inert material and put the spilled material in an appropriate waste disposal. Finish cleaning by spreading water on the contaminated surface and allow to evacuate through the sanitary system.

\section{Section 7: Handling and Storage}

Precautions:

Keep away from heat. Keep away from sources of ignition. Empty containers pose a fire risk, evaporate the residue under a fume hood. Ground all equipment containing material. Do not ingest. Do not breathe gas/fumes/ vapour/spray. Wear suitable protective clothing In case of insufficient ventilation, wear suitable respiratory equipment If ingested, seek medical advice immediately and show the container or the label. Avoid contact with skin and eyes 
Storage:

Keep container dry. Keep in a cool place. Ground all equipment containing material. Keep container tightly closed. Keep in a cool, well-ventilated place. Combustible materials should be stored away from extreme heat and away from strong oxidizing

agents.

\section{Section 8: Exposure Controls/Personal Protection}

Engineering Controls:

Provide exhaust ventilation or other engineering controls to keep the airborne concentrations of vapors below their respective threshold limit value. Ensure that eyewash stations and safety showers are proximal to the work-station location.

Personal Protection:

Splash goggles. Lab coat. Vapor respirator. Be sure to use an approved/certified respirator or equivalent. Gloves.

Personal Protection in Case of a Large Spill:

Splash goggles. Full suit. Vapor respirator. Boots. Gloves. A self contained breathing apparatus should be used to avoid inhalation of the product. Suggested protective clothing might not be sufficient; consult a specialist BEFORE handling this product.

Exposure Limits: Not available.

Section 9: Physical and Chemical Properties

Physical state and appearance: Liquid.

Odor: Odorless.

Taste: Not avalable.

Molecular Weight: $102.09 \mathrm{~g} / \mathrm{mole}$

Color: Colorless.

pH ( $1 \%$ soln/water): Not available.

Boiling Point: $241.7^{\circ} \mathrm{C}\left(467.1^{\circ} \mathrm{F}\right)$

Melting Point: $-49.2^{\circ} \mathrm{C}\left(-56.6^{\circ} \mathrm{F}\right)$

Critical Temperature: Not available.

Specific Gravity: 1.205 (Water $=1)$

Vapor Pressure: $0.03 \mathrm{~mm}$ of $\mathrm{Hg}\left(@ 20^{\circ} \mathrm{C}\right)$

Vapor Density: $3.52($ Air $=1)$

Volatility: Not available.

Odor Threshold: Not available.

Water/Oil Dist. Coeff.: Not available.

Ionicity (in Water): Not avalable.

Dispersion Properties: See solubility in water.

Solubility: Soluble in cold water.

Section 10: Stability and Reactivity Data

Stability: The product is stable 
Instability Temperature: Not available.

Conditions of Instability: Not available.

Incompatibility with various substances: Not available.

Corrosivity: Non-corrosive in presence of glass.

Special Remarks on Reactivity: Not avalable.

Special Remarks on Corrosivity: Not available.

Polymerization: No.

\section{Section 11: Toxicological Information}

Routes of Entry: Eye contact. Inhalation. Ingestion.

Toxicity to Animals:

Acute oral toxicity (LD50): $20700 \mathrm{mg} / \mathrm{kg}$ [Mouse]. Acute dermal toxicity (LD50): $20001 \mathrm{mg} / \mathrm{kg}$ [Rabbit].

Chronic Effects on Humans: Not available.

Other Toxic Effects on Humans: Hazardous in case of skin contact (irritant), of ingestion, of inhalation.

Special Remarks on Toxicity to Animals: Not available.

Special Remarks on Chronic Effects on Humans: Not available.

Special Remarks on other Toxic Effects on Humans: Not avaliable.

\section{Section 12: Ecological Information}

Ecotoxicity: Not avalable.

BOD5 and COD: Not avalable.

Products of Biodegradation:

Possibly hazardous short term degradation products are not likely. However, long term degradation products may arise.

Toxicity of the Products of Biodegradation: The products of degradation are more toxic.

Special Remarks on the Products of Biodegradation: Not avalable.

Section 13: Disposal Considerations

Waste Disposal:

\section{Section 14: Transport Information}

DOT Classification: Not a DOT controlled material (United States).

Identification: Not applicable.

Special Provisions for Transport: Not applicable.

Section 15: Other Regulatory Information

Federal and State Regulations: TSCA 8(b) inventory. Propylene carbonate 
Other Regulations: Not available.

Other Classifications:

WHMIS (Canada): Not controlled under WHMIS (Canada).

DSCL (EEC): R36/38- Irritating to eyes and skin.

HMIS (U.S.A.):

Health Hazard: 1

Fire Hazard: 1

Reactivity: 0

Personal Protection: h

National Fire Protection Association (U.S.A.):

Health: 1

Flammability: 1

Reactivity: 0

Specific hazard:

Protective Equipment:

Gloves. Lab coat. Vapor respirator. Be sure to use an approved/certified respirator or equivalent. Splash goggles.

Section 16: Other Information

References: Not available.

Other Special Considerations: Not available.

Created: 10/10/2005 08:24 PM

Last Updated: 05/21/2013 12:00 PM

The information above is believed to be accurate and represents the best information currently available to us. However, we make no warranty of merchantability or any other warranty, express or implied, with respect to such information, and we assume no liability resulting from its use. Users should make their own investigations to determine the suitability of the information for their particular purposes. In no event shall Science Lab.com be liable for any claims, losses, or damages of any third party or for lost profits or any special, indirect, incidental, consequential or exemplary damages, howsoever arising, even if ScienceLab.com has been advised of the possibility of such damages. 


\section{Material Safety Data Sheet}

\section{Lithium perchlorate anhydrous MSDS}

\begin{tabular}{|c|c|}
\hline \multicolumn{2}{|c|}{ Section 1: Chemical Product and Company Identification } \\
\hline Product Name: Lithium perchlorate anhydrous & Contact Information: \\
\hline Catalog Codes: SLL1354 & Sciencelab.com, Inc. \\
\hline CAS\#: $7791-03-9$ & $\begin{array}{l}14025 \text { Smith Rd. } \\
\text { Houston, Texas } 77396\end{array}$ \\
\hline RTECS: SC8750000 & US Sales: $1-800-901-7247$ \\
\hline TSCA: TSCA 8 (b) inventory: Lithium perchlorate anhydrous & International Sales: 1-281-441-4400 \\
\hline 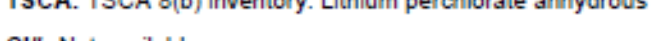 & Order Online: ScienceLab.com \\
\hline $\mathrm{Cl} \#$ : Not available. & CHEMTREC (24HR Emergency Telephone), call: \\
\hline Synonym: & $1-800-424-9300$ \\
\hline Chemical Name: Not available. & International CHEMTREC, call: 1-703-527-3887 \\
\hline Chemical Formula: $\mathrm{LiClO}_{4}$ & For non-emergency assistance, call: $1-281-441-4400$ \\
\hline
\end{tabular}

Section 2: Composition and Information on Ingredients

Composition:

\begin{tabular}{|l|l|l|}
\hline Name & CAS \# & \% by Weight \\
\hline Lithium perchlorate anhydrous & $7791-03-9$ & 100 \\
\hline
\end{tabular}

Toxicological Data on Ingredients: Lithium perchlorate anhydrous LD50: Not available. LC50: Not available.

\section{Section 3: Hazards Identification}

Potential Acute Health Effects:

Very hazardous in case of skin contact (corrosive, irritant), of eye contact (irritant), of ingestion, of inhalation. The amount of tissue damage depends on length of contact. Eye contact can result in corneal damage or blindness. Skin contact can produce inflammation and blistering. Inhalation of dust will produce irritation to gastro-intestinal or respiratory tract, characterized by burning, sneezing and coughing. Severe over-exposure can produce lung damage, choking, unconsciousness or death. Prolonged exposure may result in skin burns and ulcerations. Over-exposure by inhalation may cause respiratory irritation. Inflammation of the eye is characterized by redness, watering, and itching. Skin inflammation is characterized by itching. scaling, reddening. or, occasionally, blistering.

Potential Chronic Health Effects:

Very hazardous in case of skin contact (corrosive, irritant), of eye contact (irritant), of ingestion, of inhalation.

CARCINOGENIC EFFECTS: Not available. MUTAGENIC EFFECTS: Not available. TERATOGENIC EFFECTS: Not available DEVELOPMENTAL TOXICITY: Not available. The substance is toxic to the nervous system. Repeated or prolonged exposure to the substance can produce target organs damage. Repeated exposure of the eyes to a low level of dust can produce eye irritation. Repeated skin exposure can produce local skin destruction, or dermatitis. Repeated inhalation of dust can 
produce varying degree of respiratory irritation or lung damage. Repeated or prolonged inhalation of dust may lead to chronic respiratory irritation.

\section{Section 4: First Aid Measures}

Eye Contact:

Check for and remove any contact lenses. Immediately flush eyes with running water for at least 15 minutes, keeping eyelids open. Cold water may be used. Do not use an eye ointment. Seek medical attention.

Skin Contact:

If the chemical got onto the clothed portion of the body, remove the contaminated clothes as quickly as possible, protecting your own hands and body. Place the victim under a deluge shower. If the chemical got on the victim's exposed skin, such as the hands: Gently and thoroughly wash the contaminated skin with running water and non-abrasive soap. Be particularly careful to clean folds, crevices, creases and groin. Cold water may be used. If irritation persists, seek medical attention. Wash contaminated clothing before reusing.

Serious Skin Contact:

Wash with a disinfectant soap and cover the contaminated skin with an anti-bacterial cream. Seek medical attention.

Inhalation: Allow the victim to rest in a well ventilated area. Seek immediate medical attention.

Serious Inhalation:

Evacuate the victim to a safe area as soon as possible. Loosen tight clothing such as a collar, tie, belt or waistband. If breathing is difficult, administer oxygen. If the victim is not breathing. perform mouth-to-mouth resuscitation. WARNING: It may be hazardous to the person providing aid to give mouth-to-mouth resuscitation when the inhaled material is toxic, infectious or corrosive. Seek immediate medical attention.

Ingestion:

Do not induce vomiting. Loosen tight clothing such as a collar, tie, belt or waistband. If the victim is not breathing, perform mouth-to-mouth resuscitation. Seek immediate medical attention.

Serious Ingestion: Not avalable.

\section{Section 5: Fire and Explosion Data}

Flammability of the Product: Non-flammable.

Auto-Ignition Temperature: Not applicable.

Flash Points: Not applicable.

Flammable Limits: Not applicable.

Products of Combustion: Not avalable.

Fire Hazards in Presence of Various Substances: Not applicable.

Explosion Hazards in Presence of Various Substances:

Risks of explosion of the product in presence of mechanical impact: Not available. Risks of explosion of the product in presence of static discharge: Not avallable. Slightly explosive to explosive in presence of combustible materials.

Fire Fighting Media and Instructions: Not applicable.

Special Remarks on Fire Hazards: Not available.

Special Remarks on Explosion Hazards: Not avalable.

\section{Section 6: Accidental Release Measures}

Small Spill: Use appropriate tools to put the spilled solid in a convenient waste disposal container

Large Spill: 
Oxidizing material. Corrosive solid. Stop leak if without risk. Do not get water inside container. Avoid contact with a

combustible material (wood, paper, oil, clothing...). Keep substance damp using water spray. Do not touch spilled material.

Use water spray to reduce vapors. Prevent entry into sewers, basements or confined areas; dike if needed. Call for assistance on disposal.

\section{Section 7: Handling and Storage}

Precautions:

Keep container dry. Keep away from heat. Keep away from sources of ignition. Keep away from combustible material Do not breathe dust. Never add water to this product In case of insufficient ventilation, wear suitable respiratory equipment If you feel unwell, seek medical attention and show the label when possible. Avoid contact with skin and eyes

Storage: Corrosive materials should be stored in a separate safety storage cabinet or room.

\section{Section 8: Exposure Controls/Personal Protection}

Engineering Controls:

Use process enclosures, local exhaust ventilation, or other engineering controls to keep airborne levels below recommended exposure limits. If user operations generate dust, fume or mist, use ventilation to keep exposure to airborne contaminants below the exposure limit.

Personal Protection:

Splash goggles. Lab coat. Vapor and dust respirator. Be sure to use an approved/certified respirator or equivalent. Gloves.

Personal Protection in Case of a Large Spill:

Splash goggles. Full suit. Vapor and dust respirator. Boots. Gloves. A self contained breathing apparatus should be used to

avoid inhalation of the product. Suggested protective clothing might not be sufficient; consult a specialist BEFORE handling this product.

Exposure Limits: Not available.

\section{Section 9: Physical and Chemical Properties}

Physical state and appearance: Solid. (Crystalline solid.)

Odor: Not avalable.

Taste: Not avalable.

Molecular Weight: $106.39 \mathrm{~g} / \mathrm{mole}$

Color: White.

pH (1\% soln/water): Not available.

Boiling Point: Decomposes.

Melting Point: $236^{\circ} \mathrm{C}\left(456.8^{\circ} \mathrm{F}\right)$

Critical Temperature: Not available.

Specific Gravity: $2.429($ Water $=1)$

Vapor Pressure: Not applicable.

Vapor Density: Not avalable.

Volatility: Not available.

Odor Threshold: Not available.

Water/Oil Dist. Coeff.: Not available. 
Ionicity (in Water): Not available.

Dispersion Properties: See solubility in water, methanol, diethyl ether, acetone.

Solubility: Soluble in cold water, methanol, diethyl ether, acetone.

\section{Section 10: Stability and Reactivity Data}

Stability: The product is stable.

Instability Temperature: Not available.

Conditions of Instability: Not available.

Incompatibility with various substances: Not available.

Corrosivity: Non-corrosive in presence of glass.

Special Remarks on Reactivity: Not avalable.

Special Remarks on Corrosivity: Not available.

Polymerization: No.

\section{Section 11: Toxicological Information}

Routes of Entry: Eye contact. Inhalation. Ingestion.

Toxicity to Animals:

LD50: Not available. LC50: Not available.

Chronic Effects on Humans: The substance is toxic to the nervous system.

Other Toxic Effects on Humans: Very hazardous in case of skin contact (corrosive, irritant), of ingestion, of inhalation.

Special Remarks on Toxicity to Animals: Not available.

Special Remarks on Chronic Effects on Humans: Human: passes through the placenta, excreted in maternal milk.

Special Remarks on other Toxic Effects on Humans: Not avalable.

\section{Section 12: Ecological Information}

Ecotoxicity: Not avalable.

BOD5 and COD: Not avalable.

Products of Biodegradation:

Possibly hazardous short term degradation products are not likely. However, long term degradation products may arise.

Toxicity of the Products of Biodegradation: The products of degradation are more toxic.

Special Remarks on the Products of Biodegradation: Not avalable.

Section 13: Disposal Considerations

Waste Disposal

Section 14: Transport Information 
DOT Classification: CLASS 5.1: Oxidizing material.

Identification: : Perchlorate, inorganic, n.o.s. : UN1481 PG: II

Special Provisions for Transport: Not available.

Section 15: Other Regulatory Information

Federal and State Regulations: TSCA 8(b) inventory: Lithium perchlorate anhydrous

Other Regulations: OSHA: Hazardous by definition of Hazard Communication Standard (29 CFR 1910.1200).

Other Classifications:

WHMIS (Canada): CLASS C: Oxidizing material.

DSCL (EEC): R34- Causes burns.

HMIS (U.S.A.):

Health Hazard: 3

Fire Hazard: 0

Reactivity: 0

Personal Protection: j

National Fire Protection Association (U.S.A.):

Health: 3

Flammability: 0

Reactivity: 0

Specific hazard:

Protective Equipment:

Gloves. Lab coat. Vapor and dust respirator. Be sure to use an approved/certified respirator or equivalent. Wear appropriate respirator when ventilation is inadequate. Splash goggles.

\section{Section 16: Other Information}

References: Not available.

Other Special Considerations: Not available.

Created: 10/09/2005 05:59 PM

Last Updated: 05/21/2013 12:00 PM

The information above is believed to be accurate and represents the best information currently available to us. However, we make no warranty of merchantability or any other warranty, express or implied, with respect to such information, and we assume no liability resuiting from its use. Users should make their own investigations to determine the suitability of the information for their particular purposes. In no event shall ScienceLab.com be liable for any claims, losses, or damages of any third party or for lost profits or any special, indirect, incidental, consequential or exemplary damages, howsoever arising, even if ScienceLab.com has been advised of the possibility of such damages. 


\section{MICRO CHEM}

1254 Chestnut Street Newton, MA 02464-1418

Tel: (617) 965-5511

Fax: (617) 965-5818

\section{MATERIAL SAFETY DATA SHEET}

SECTION 1. CHEMICAL IDENTIFICATION

$\begin{array}{ll}\text { CHEMICAL NAME: } & \text { Organic Polymer Solution } \\ \text { TRADE NAME: } & \text { 950 PMMA Series Resists in Anisole } \\ & \text { Positive Radiation Sensitive Resists } \\ \text { PRODUCT \#: } & \text { See Table } 1 \text { - Section } 9\end{array}$

SECTION 2. COMPOSITION-

HAZARDOUS

INGREDIENTS: $\quad$ Anisole (CAS: 100-66-3); 85-99\% (See Table 1 - Section 9)

OTHER

Poly(methylmethacry late) (CAS: 9011-14-7)

INGREDIENTS:

SECTION 3. HAZARD DATA

\section{INFLAMMABILITY: Flammable liquid.}

SKIN CONTACT: $\quad$ May cause skin irritation.

EYE CONTACT: $\quad$ Vapor or mist is irritating to the eyes.

INHALATION: $\quad$ Vapor or mist is irritating to mucous membranes and upper respiratory tract.

MUTAGENICITY: Not known to be mutagenic.

CARCINOGENICITY: Not known to be carcinogenic.

TARGET ORGANS: $\quad$ Eyes, Skin, Respiratory System, and Nervous System.

SECTION 4. FIRST AID MEASURES-

INHALATION: $\quad$ Move the affected person away from the contaminated area and into the fresh air. If necessary seek medical advice.

$\begin{array}{ll}\text { INGESTION: } & \text { Rinse mouth out with water. Consult a doctor if necessary. } \\ \text { SKIN CONTACT: } & \text { Rinse with plenty of water. }\end{array}$

EYE CONTACT: $\quad$ Rinse with water while keeping the eyes wide open.

SECTION 5. FIRE FIGHTING MEASURES-

EXTINGUISHING MEDIA: SPECIAL FIRE FIGHTING PRECAUTIONS:

UNUSUAL FIRE OR EXPLOSION HAZARDS:
Dry chemical, carbon dioxide.

Wear self-contained breathing apparatus (SCBA) and personal protective equipment to prevent contact with skin and eyes.

Heat will build pressure and may rupture closed containers. Keep containers cool with water spray. 
MATERIAL SAFETY DATA SHEET

PAGE 2

CHEMICAL NAME:
TRADE NAME:

Organic Polymer Solution

03 March 2005

PMMA Series Resists in Anisole

PRODUCT \#

Positive Radiation Sensitive Resists

See Table 1 - Section 9

SECTION 6. ACCIDENTAL RELEASE PROCEDURES

EVACUATION

PROCEDURES \& SAFETY: Wear appropriate protective gear for the situation. See Personal Protection information in Section 8. Eliminate all sources of ignition. Do not breathe vapors or dust.

CLEANUP \& DISPOSAL

OF SPILI

Absorb with an inert absorbent. Sweep up and place in an appropriate closed container. Use clean, non-sparking tools to collect absorbed material.

ENVIRONMENTAL \&

REGULATORY REPORTING: Do not flush to drain. If required proper authorities should be notified.

SECTION 7. STORAGE AND HANDLING PRECAUTIONS

STORAGE: $\quad$ Store in tightly closed container in a cool, dry, well-ventilated

environment away from ignition sources.

HANDLING: $\quad$ Use only under yellow light.

Keep away from heat, sparks, and flames.

Use only with mechanical exhaust.

Do not contact with skin, eyes, and clothing

Avoid prolonged or repeated contact with skin.

Do not breathe vapors or mist.

Wash with soap and water after handling.

Have safety shower and eye wash available.

Store and transfer under a blanket of dry inert gas.

SECTION 8. EXPOSURE CONTROLS AND PERSONAL PROTECTION_

RESPIRATORY

PROTECTION:

VENTILATION:

Under normal conditions, use of air-purifying (half-mask/full-face) respirator with cartridges/canisters approved for use against organic vapors, dust, mists and fumes is recommended.

General area dilution/exhaust ventilation.

SKINPROTECTION: PVC protective gloves are highly recommended.

EYE PROTECTION: Safety goggles are highly recommended.

MicroChem Corp 1254 Chestnut Street Newton, MA 02464 1418*Tel:(617)965-5511•Fas:(617)965-5818 
CHEMICAL NAME: TRADE NAME:

Organic Polymer Solution

PRODUCT \#:

950PMMA Series Resists in Anisole

Positive Radiation Sensitive Resists

See Table 1 - Section 9

SECTION 9. PHYSICAL AND CHEMICAL DATA

\section{APPEARANCE:}

ODOR:

BOILING POINT:

SPECIFIC GRAVITY:

VAPOR PRESSURE:

VAPOR DENSITY:

$\mathrm{H}_{2} \mathrm{O}$ SOLUBILITY:

\% VOLATILES:

EVAPORATION RATE:

FLASH POINT:

AUTOIGNITION TEMP:

EXPLOSION LIMITS:
Clear to straw colored liquid

Strong

$154^{\circ} \mathrm{C}\left(309^{\circ} \mathrm{F}\right)$

See Table 1 below

$13 \mathrm{~mm}$ (a) $42^{\circ} \mathrm{C}\left(108^{\circ} \mathrm{F}\right)$

3.7 (air=1)

Slightly soluble.

See Table 1 below

$0.1(\mathrm{BuAc}=1)$

$44^{\circ} \mathrm{C}\left(111^{\circ} \mathrm{F}\right) \mathrm{CC}$

$475^{\circ} \mathrm{C}\left(887^{\circ} \mathrm{F}\right)$

unk. Lower

unk. Upper

Table 1

Name Product \#
\begin{tabular}{|l|l|c|c|c|}
\hline 950A1 & M230001 & 0.994 & 99 & 985 \\
\hline 950A2 & M230002 & 0.996 & 98 & 975 \\
\hline 950A3 & M230003 & 0.998 & 97 & 970 \\
\hline 950A4 & M230004 & 1.000 & 96 & 960 \\
\hline 950A4.5 & M230504 & 1.001 & 95.5 & 958 \\
\hline 950A5 & M230005 & 1.004 & 95 & 955 \\
\hline 950A5.5 & M230505 & 1.004 & 94.5 & 950 \\
\hline 950A6 & M230006 & 1.005 & 94 & 945 \\
\hline 950A7 & M230007 & 1.007 & 93 & 935 \\
\hline 950A8 & M230008 & 1.009 & 92 & 930 \\
\hline 950A9 & M230009 & 1.010 & 91 & 920 \\
\hline 950A10 & M230010 & 1.012 & 90 & 910 \\
\hline 950A11 & M230011 & 1.014 & 89 & 900 \\
\hline 950A12 & M230012 & 1.016 & 88 & 895 \\
\hline 950A13 & M230013 & 1.018 & 87 & 885 \\
\hline 950A15 & M230015 & 1.022 & 85 & 870 \\
\hline
\end{tabular}

SECTION 10. REACTIVITY DATA

STABILITY: Stable

INCOMPATIBILITY: $\quad$ Strong Oxidizing Agents, Strong Acids, Strong Bases

HAZARDOUS COMBUSTION OR

DECOMPOSITION PRODUCTS: Carbon Monoxide, Carbon Dioxide, Phenol

MicroChem Corp 1254 Chestnut Street*Newton, MA 02464 1418*Tel:(617)965-5511*Fax:(617)965-5818 
MATERIAL SAFETY DATA SHEET

PAGE 4

CHEMICAL NAME: Organic Polymer Solution

03 March 2005

TRADE NAME:

950PMMA Series Resists in Anisole

PRODUCT \#

Positive Radiation Sensitive Resists

See Table 1 - Section 9

SECTION 11. TOXICITY HAZARDS

ACUTE TOXICITY:

As Anisole:

ORAL:

LD50 (rat): $\quad 3700 \mathrm{mg} / \mathrm{kg}$

SKIN:

LD50 (rat): $\quad>5000 \mathrm{mg} / \mathrm{kg}$

LOCAL EFFECTS: $\quad$ Slightly irritating to the skin. Not irritating to rabbits on ocular application. No sensitizing reaction was observed for guinea pigs.

SECTION 12. ECOLOGICAL DATA

BIODEGRADABILITY: Readily biodegradable.

BIOACCUMULATION: Not potentially bioaccumulable.

ECOTOXICITY: $\quad$ EC50 (Daphnia/24 hr): $40 \mathrm{mg} / \mathrm{l}$

SECTION 13. DISPOSAL CONSIDERATIONS-

Comply with applicable local, state or international regulations regarding the proper disposal of this material and/or containers.

SECTION 14. TRANSPORTATION INFORMATION

HAZARD CLASSIFICATION: Flammable Liquid

SHIPPING NAME: Resin Solution

UN NUMBER:

UN 1866

PACKING GROUP

III

\section{SECTION 15. REGULATORY INFORMATION}

\section{EUROPEAN INFORMATION}

EINECS LISTED

EC Nos: Anisole 202-876-1

RISK \& SAFETY PHRASES

R10 Flammable

S16 Keep away from sources of ignition. No smoking.

S24 Avoid contact with skin.

INDICATIONS OF DANGER

None 
MATERIAL SAFETY DATA SHEET

CHEMICAL NAME: TRADE NAME:

Organic Polymer Solution

PRODUCT \#: 950PMMA Series Resists in Anisole

Positive Radiation Sensitive Resists See Table 1 - Section 9

\section{US AND INTERNATIONAL INFORMATION}

HAZARDOUS LISTINGS: $\quad$ All ingredients appear on the TSCA Inventory of Chemical Substances, EINECS, Canadian DSL and the Japan ENCS Listing.

SARA Title III:

This product IS NOT subject to SARA Title III, Section 313 Reporting Requirements.

Calif. SCAQMD Rule 443.1 VOC's: See Table 1 - Section 9

\section{SECTION 16. ADDITIONAL PRECAUTIONS AND COMMENTS-}

National Fire Protection Association Hazard Ratings - NFPA:

1 Health Hazard Rating

2 Flammability Rating

0 Reactivity Rating

To the best of our knowledge, the above information is believed to be accurate but does not claim to be all-inclusive and is intended to be used only as a guide. The supplier makes no warranty of any kind, expressed or implied, conceming the use of this product and shall not be held liable for any damage resulting from handling or from contact with the above product. User assumes all risks incident to its use.

MSDS Revision Information:

A) Removed "NANO" name from trade name.

B) General update of MSDS. Added European Risk and Safety phrases to Section 15. 


\section{1-Ethyl-3-methylimidazolium tetrafluoroborate: sc- 237548

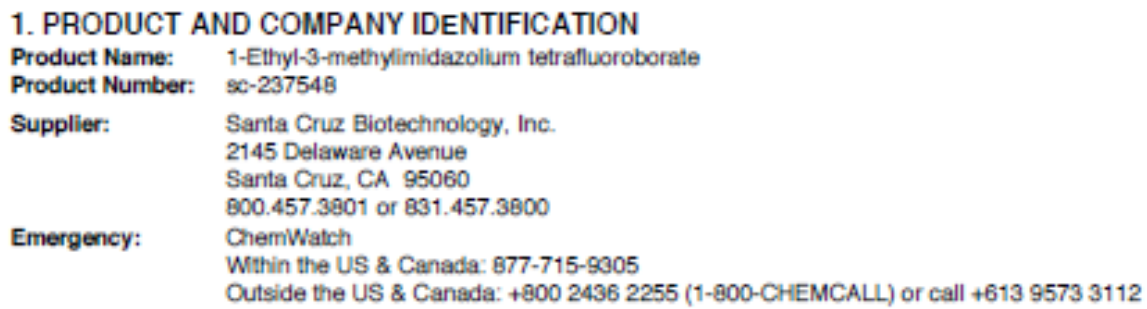

\section{HAZARDS IDENTIFICATION}

\section{Emergency Overview}

OSHA Hazards

Toxic by ingestion.

GHS Claseification

Acute toxicity, Oral (Category 4)

Acute toxicily, Dermal (Category 5)

Acute aquatic toxicily (Category 2)

GHS Label elements, including precautionary statements Piclogram

Signal word

Hazard statement(s)

$\mathrm{H} 302$

$\mathrm{H} 313$

H401

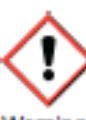

Warning

Harmful if swallowed.

May be harmful in contact with skin.

Toxic to aquatic life.

Precautionary statement(s)

$$
\text { none }
$$

HMIS Classification

Health hazard: 2

Flammability:

Physical hazards: 0

NFPA Rating

Health hazard: 2

Fire:

Reactivity Hazard: 0

Potential Health Effects

Inhalation: May be harmful it inhaled. May cause respiratory tract irritation.

Skin: $\quad$ May be harmful if absorbed through skin. May cause skin irritation.

Eyee: $\quad$ May cause eye irritation.

Ingestion: Toxic if swallowed.

\section{COMPOSITION/NFORMATION ON INGREDIENTS}

Synonyme: $\quad$ EMIMBF4

Formula: $\quad$ CoH11BF4N2 
Molecular Weight: 197.97

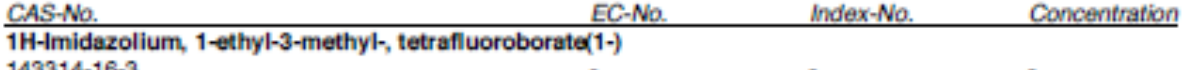

$143314-16-3$

\section{FIRST AID MEASURES}

General advice

Consult a physician. Show this safety data sheet to the doctor in attendance. Move out of dangerous area.

If inhaled

II breathed in, move person into fresh air. If not breathing. give artificial respiration. Consult a physician.

In case of akin contact

Wash off with soap and plenty of water. Consult a physician.

In case of eye contact

Flush eyes with water as a precaution.

If ewallowed

Never give anything by mouth to an unconscious person. Rinse mouth with water. Consult a physician.

\section{FIREFIGHTING MEASURES}

Conditions of flammability

Not flammable or combustible.

Suitable extinguishing medis

Use water spray, alcohol-resistant foam, dry chemical or carbon dioxide.

Special protective equipment for firefighters

Wear self contained breathing apparatus for fire fighting if necessary.

Hazardous combustion products

Hazardous decomposition products formed under fire conditions - Carbon oxides, nitrogen oxides (NOx).

hydrogen fluoride, borane/boron oxides

\section{ACCIDENTAL RELEASE MEASURES}

Personal precautions

Use personal protective equipment. Avoid breathing vapors, mist or gas. Ensure adequate ventilation.

Environmental precautions

Prevent further leakage or spillage if sate to do so. Do not let product enter drains. Discharge into the

environment must be avoided.

Methods and materials for containment and cleaning up

Soak up with inert absorbent material and dispose of as hazardous waste. Keep in suitable, closed containers for disposal.

\section{HANDLING AND STORAGE}

Precautions for safe handling

Avoid contact with skin and eyes. Avoid inhalation of vapor of mist.

Conditions for eafe storage

Keep container tightly closed in a dry and well-ventlated place. Containers which are opened must be carefully

resealed and kept upright to prevent leakage. Store at room temperature. Hygrosoopic. Store under inert gas.

\section{EXPOSURE CONTROLS/PERSONAL PROTECTION}

Contains no substances with occupational exposure limit values.

Personal protective equipment

Respiratory protection

Where risk assessment shows air-purifying respirators are appropriate use a full-face respirator with multi-

purpose combination (US) or type ABEK (EN 14387) respirator cartridges as a backup to engineering controls. If

the respirator is the sole means of protection, use a full-lace supplied air respirator. Use respirators and

components tested and approved under appropriate government standards such as NIOSH (US) or CEN (EU). 


\begin{tabular}{|c|c|c|c|}
\hline \multicolumn{4}{|c|}{$\begin{array}{l}\text { Handle with gloves. Gloves must be inspected prior to use. Use proper glove removal technique (without touching } \\
\text { glove's outer surface) to avoid skin contact with this product. Dispose of contaminated gloves after use in } \\
\text { accordance with applicable laws and good laboratory practices. Wash and dry hands. }\end{array}$} \\
\hline \multicolumn{4}{|l|}{ Eye protection } \\
\hline \multicolumn{4}{|c|}{$\begin{array}{l}\text { Face shield and safety glasses Use equipment for eye protection tested and approved under appropriate } \\
\text { government standards such as NIOSH (US) or EN } 166(E \mathrm{E}) \text {. }\end{array}$} \\
\hline \multicolumn{4}{|c|}{ Skin and body protection } \\
\hline \multicolumn{4}{|c|}{$\begin{array}{l}\text { Complete suit protecting against chemicals. The type of protective equipment must be selected according to the } \\
\text { concentration and amount of the dangerous substance at the specific workplace. }\end{array}$} \\
\hline \multicolumn{4}{|l|}{ Hygiene measures } \\
\hline \multicolumn{4}{|c|}{$\begin{array}{l}\text { Handle in accordance with good industrial hygiene and safety practice. Wash hands before breaks and at the } \\
\text { end of workday. }\end{array}$} \\
\hline \multicolumn{4}{|c|}{ 9. PHYSICAL AND CHEMICAL PROPERTIES } \\
\hline Form & liquid & $\mathrm{pH}$ & no data available \\
\hline Melting pointfreezing point & $15^{\circ} \mathrm{C}\left(59^{\circ} \mathrm{F}\right)$ & Ignition temperature & no data available \\
\hline Boiling point & $>350^{\circ} \mathrm{C}\left(>662^{\circ} \mathrm{F}\right)$ & Autoigniltion temperature & no data available \\
\hline Lower explosion limit & no data available & Upper explosion Imit & no data available \\
\hline Vapor pressure & no data available & Water solubility & no data available \\
\hline Relative vapor density & no data available & Odor & no data available \\
\hline Odor Threshold & no data available & Evaporation rate & no data available \\
\hline Flash point & $\begin{array}{l}113^{\circ} \mathrm{C}\left(235^{\circ} \mathrm{F}\right)- \\
\text { closed cup }\end{array}$ & Density & $\begin{array}{l}1.294 \mathrm{~g} / \mathrm{mL} \text { at } \\
25^{\circ} \mathrm{C}\left(77^{\circ} \mathrm{F}\right)\end{array}$ \\
\hline $\begin{array}{l}\text { Partition coefficient: } \\
\text { n-octanol/water }\end{array}$ & no data available & & \\
\hline
\end{tabular}

10. STABILITY AND REACTIVITY

Chemical stability

Stable under recommended storage conditions.

Poseibility of hazardous reactions

no data available

Conditione to avoid

no data avalable

Materials to avoid

Strong oxidizing agents

Hazardous decomposition products

Hazardous decomposition products formed under fire conditions - Carbon oxides, nitrogen oxides (NOx).

hydrogen fluoride, borane/boron oxides

Other decomposition products

no data avalable

\section{TOXICOLOGICAL INFORMATION}

Acute toxicity

Oral LD50: LD50 Oral - rat - > $300-2,000 \mathrm{mg} / \mathrm{kg}$

Inhalation LC50. no data available

Dermal LD50: LD50 Dermal - rat - > 2,000 mg/kg

Other information on acute toxicity: no data avalable

Skin corrosion/irritation

Skin - rabbit - No skin irritation - OECD Test Guideline 404

Serious eye damage/eye irritation

Eyes - rabbit - No eye irritation - OECD Test Guideline 405

Respiratory or skin sensitization

no data avalable

Germ cell mutagenicity

no data avaliable 


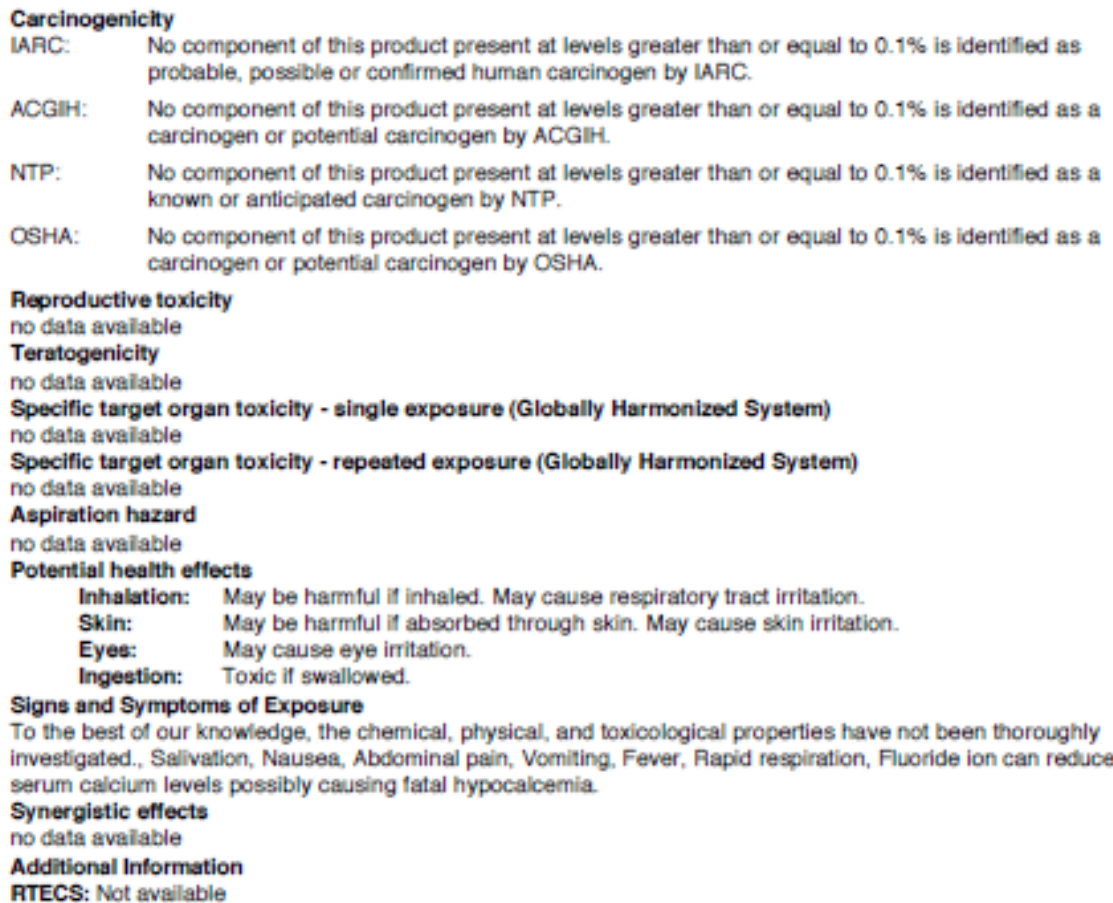

ACGIH: No component of this product present at levels greater than or equal to $0.1 \%$ is identified as a carcinogen or potential carcinogen by ACGIH.

NTP: $\quad$ No component of this product present at levels greater than or equal to $0.1 \%$ is identified as a known of anticipated carcinogen by NTP.

OSHA: No component of this product present at levels greater than or equal to $0.1 \%$ is identified as a carcinogen or potential carcinogen by OSHA.

Reproductive toxicity

no data avalable

Teratogenicity

no data avalable

Specific target organ toxicity - single exposure (Globally Harmonized System)

no data avalable

Specific target organ toxicity - repeated exposure (Globally Harmonized System)

no data avalable

Aspiration hazard

no data avalable

Potential health effects

Inhalation: May be harmful if inhaled. May cause respiratory tract irritation.

Skin: May be harmful if absorbed through skin. May cause skin irritation.

Eyee: $\quad$ May cause eye irritation.

Ingeetion: Toxic if swallowed.

Signs and Symptoms of Exposure

To the best of our knowledge, the chemical, physical, and toxicological properties have not been thoroughly investigated., Salivation, Nausea. Abdominal pain, Vomiting. Fever, Rapid respiration, Fluoride ion can reduce serum calcium levels possibly causing fatal hypocalcemia.

Synergistic effects

no data avalable

Additional Information

RTECS: Not avellable

\section{ECOLOGICAL INFORMATION}

PBT and VPVB assessment

Bioaccumulative potential Mobility in soil

no data available no data available

Toxicity

Toxicity to fish: LC50 - Danio rerio (zebra fish) - >101.0 mg/ - $96.0 \mathrm{~h}$

Method: OECD Test Guideline 203

Toxicity to daphnia and other aquatic invertebrates:

LC50 - Daphnia magna (Water flea) - $6.3 \mathrm{mgh}-48 \mathrm{~h}$ Method: OECD Test Guideline 202

Other adverse effects

Toxic to aquatic life. An environmental hazard cannot be excluded in the event of unprofessional handling or disposal.

\section{DISPOSAL CONSIDERATIONS}

Product

Offer surplus and non-recyclable solutions to a licensed disposal company. Contact a licensed professional waste disposal service to dispose of this material. Dissolve or mix the material with a combustible solvent and burn in a chemical incinerator equipped with an atterburner and scrubber.

Contaminated packaging

Dispose of as unused product. 
14. TRANSPORT INFORMATION

DOT (US)

Not dangerous goods

MDG

UN number: $3082 \quad$ Class: $9 \quad$ Packing group: III EMS-No: F-A, S-F

Proper shipping name: ENVIAONMENTALYY HAZARDOUS SUBSTANCE, LIOUID, N.O.S. (1H-Imidazolium, 1-

ethyl-3- methyl-, tetrafluoroborate(1-))

Marine pollutant: Marine pollutant

UTA

UN number: 3082 Class: 9 Packing group: III

Proper shipping name: Environmentally hazardous substance, liquid, n.o.s. (1H-Imidazolium, 1-ethyl-3-methyl-

tetrafluoroborate(1-))

Further information

EHS-Mark required (ADA 2.2.9.1.10, IMDG code 2.10.3) for single packagings and combination packagings containing inner packagings with Dangerous Goods > 5L. for liquids of $>5 \mathrm{~kg}$ for solids.

\section{REGULATORY INFORMATION}

OSHA Hazards

Toxic by ingestion

SARA 302 Components

SARA 302: No chemicals in this material are subject to the reporting requirements of SARA Title III, Section 302 SARA 313 Componerts

SARA 313: This material does not contain any chemical components with known CAS numbers that exceed the threshold (De Minimis) reporting levels established by SARA Title III, Section 313.

SARA $311 / 312$ Hazard

Acute Health Hazard

Maseachusetts Right To Know Components

No components are subject to the Massachusetts Right to Know Act.

Pennsylvania Right To Know Components

1H-Imidazolium, 1-ethyl-3-methyl-, tetrafluoroborate(1-)

CAS-No: $143314-16-3$

New Jersey Right To Know Components

1H-Imidazolium, 1-ethyl-3-methyl-, tetrafluoroborate(1-)

CAS-No.: $143314-16-3$

California Prop. 65 Components

This product does not contain any chemicals known to State of Calilomia to cause cancer, birth delects, or any other reproductive harm.

\section{OTHER INFORMATION}

The above information is believed to be correct but does not purport to be complete and should be used only as a guide. The burden of safe use of this material rests entirely with the user. 
MOSIS WAFER ELECTRICAL TESTS

RUN : V37P

TECHNOLOGY : SCN05
VENDOR: AMIS (ON-SEMI)

FEATURE SIZE: 0.5 microns

Run type: SHR

INTRODUCTION: This report contains the lot average results obtained by MOSIS from measurements of MOSIS test structures on each wafer of this fabrication lot. SPICE parameters obtained from similar measurements on a selected wafer are also attached.

COMMENTS : SMSCN3ME06 ON-SEMI

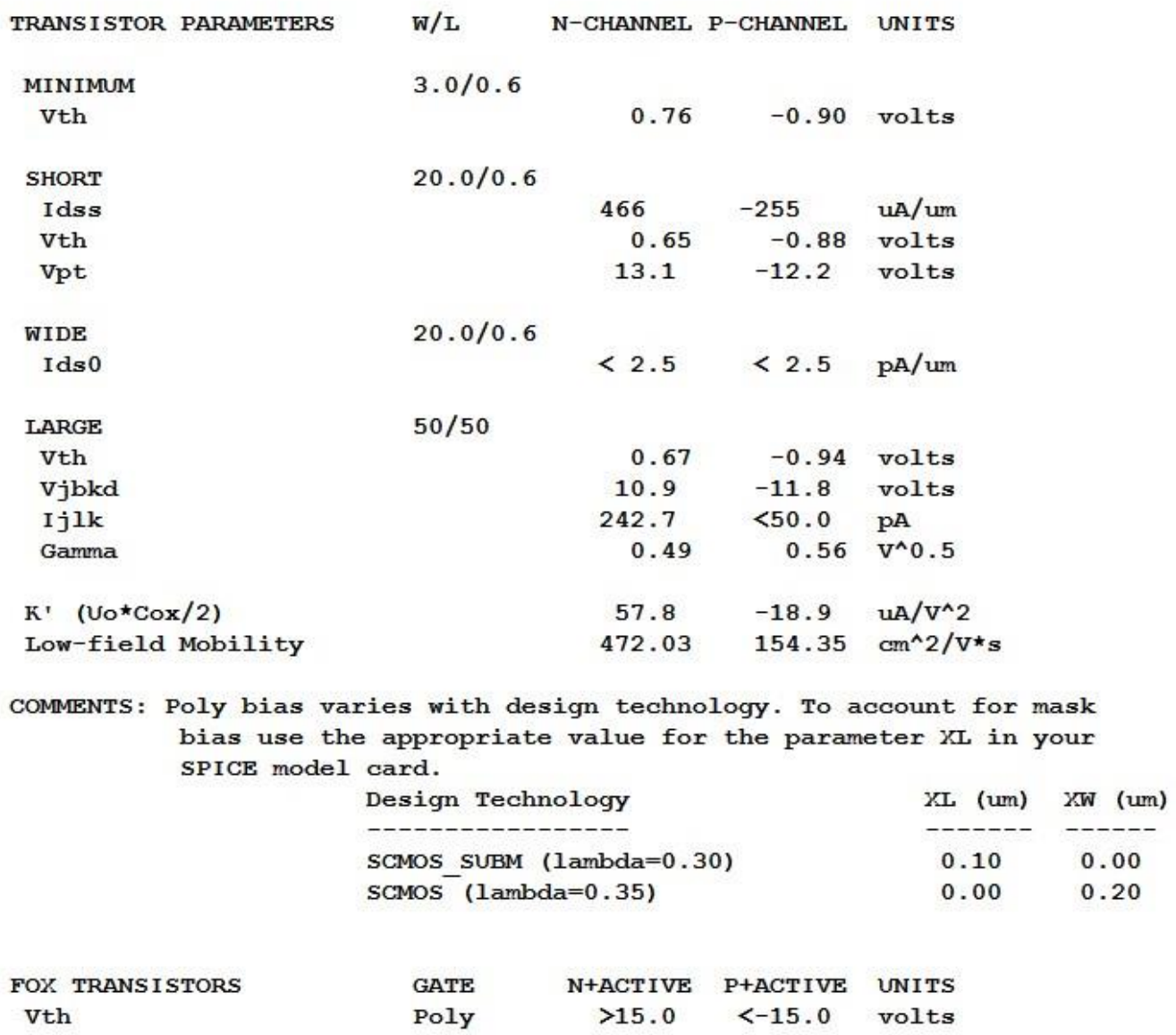


COMMENTS :

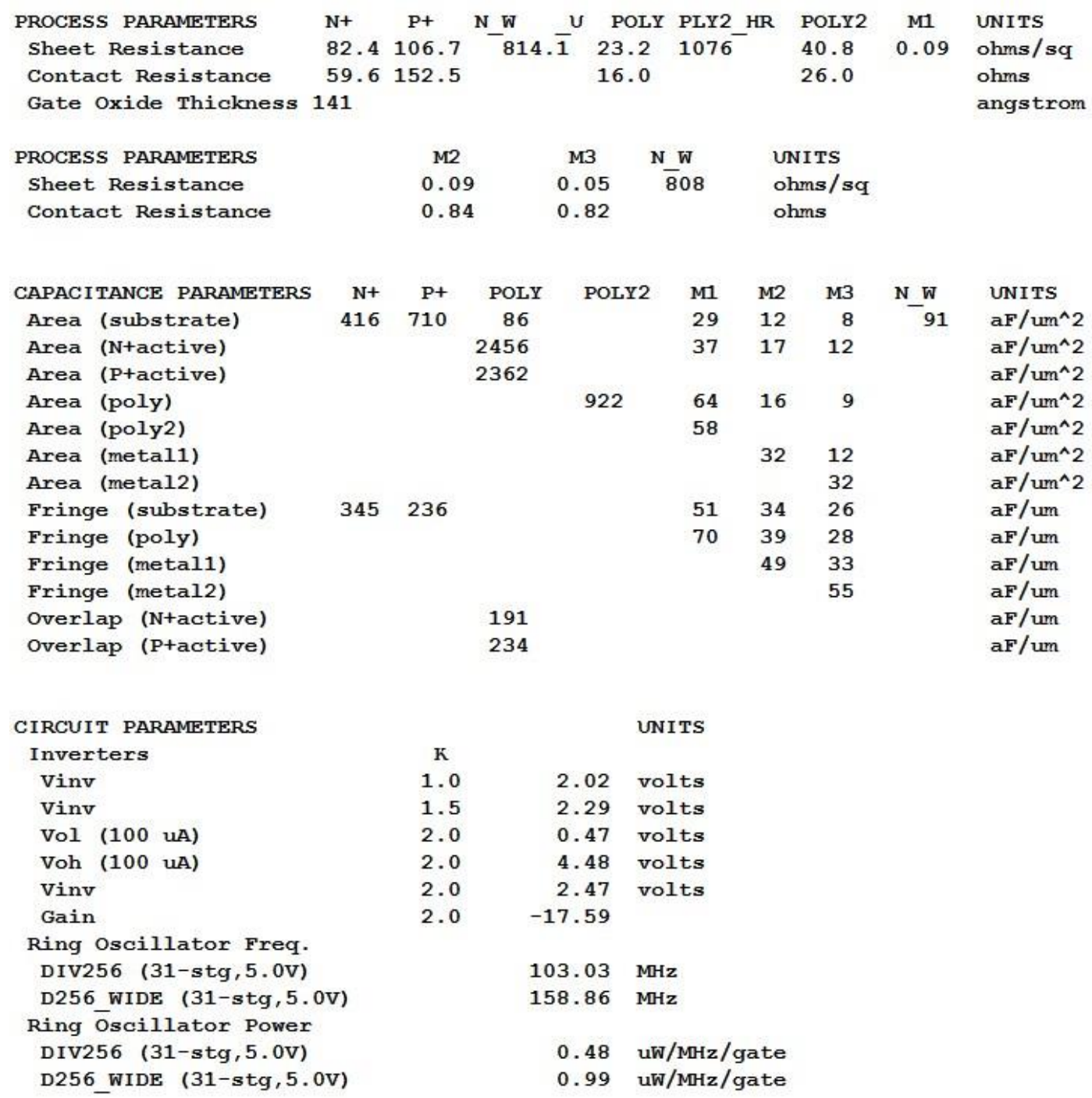

COMMENTS: SUBMICRON 
V37P SPICE BSIM3 VERSION 3.1 PARAMETERS

SPICE 3 f5 Level 8 , Star-HSPICE Level 49, UTMOST Level 8

* DATE : Oct $17 / 13$

* LOT: v37p WAF : 1003

* Temperature_parameters=Default

.MODEL CMOSN NMOS (

+ VERSION $=3.1$

$+\mathrm{XJ}=1.5 \mathrm{E}-7$

TNOM $=27$

$\mathrm{NCH}=1.7 \mathrm{E} 17$

$+\mathrm{K} 1=0.9137986$

$+\mathrm{K} 3 \mathrm{~B}=-9.7485086$

+DVTOW $=0$

+DVT0 $=0.8309419$

$+\mathrm{U}=460.0124125$

$+U \mathrm{C}=3.089014 \mathrm{E}-12$

+ AGS

+KETA $=-2.797385 \mathrm{E}-3$

$+\mathrm{RDSW}=1.115544 \mathrm{E} 3$

+ WR $\quad=1$

$+\mathrm{XL} \quad=1 \mathrm{E}-7$

$+\mathrm{DWB}=1.914595 \mathrm{E}-8$

+ CIT $=0$

+ CDSCB $=0$

+ DSUB $=0.0833302$

+ PDIBLC2 $=1.863456 \mathrm{E}-3$

+ PSCBE1 $1=3.853855 \mathrm{E} 8$

+ DELTA $=0.01$

+ PRT $=0$

+ KT1L $=0$

+ UB1 $=-7.61 \mathrm{E}-18$

$+W L \quad=0$

+ WWN $\quad=1$

+ LLN $\quad=1$

+ LWL $\quad=0$

+ CGDO $=1.91 \mathrm{E}-10$

$+\mathrm{CJ}=4.131634 \mathrm{E}-4$

$+\mathrm{CJSW}=3.400072 \mathrm{E}-10$

+ CJSWG $=1.64 \mathrm{E}-10$

$+\mathrm{CF}=0$

+ PK2 $=-0.0768747$

К2 $=-0.1071877$

พ0 $\quad=2.658488 \mathrm{E}-8$

DVT1W $=0$

DVT1 $=0.3317542$

UA $\quad=2.759471 \mathrm{E}-13$

VSAT $=1.840576 \mathrm{E} 5$

BO $=1.941274 \mathrm{E}-6$

$\mathrm{A} 1=2.420581 \mathrm{E}-5$

PRWG $\quad=0.0828351$

WINT $\quad=2.526685 \mathrm{E}-7$

XW $\quad=0$

VOFF $=-6.986376 \mathrm{E}-5$

CDSC $\quad=2.4 \mathrm{E}-4$

ETA0 $=2.045973 \mathrm{E}-3$

PCLM $=2.3615569$

PDIBLCB $=0.0644698$

PSCBE2 $=4.115782 \mathrm{E}-6$

$\mathrm{RSH}=82.4$

UTE $=-1.5$

КT2 $=0.022$

UC1 $=-5 \cdot 6 \mathrm{E}-11$

WLN $\quad=1$

WWL $\quad=0$

LW $=0$

CAPMOD $=2$

CGSO $=1.91 \mathrm{E}-10$

$\mathrm{PB} \quad=0.8399766$

PBSW $=0.809471$

PBSWG $=0.8$

PVTH0 $=-0.028514$

WKETA $=-0.0138828$

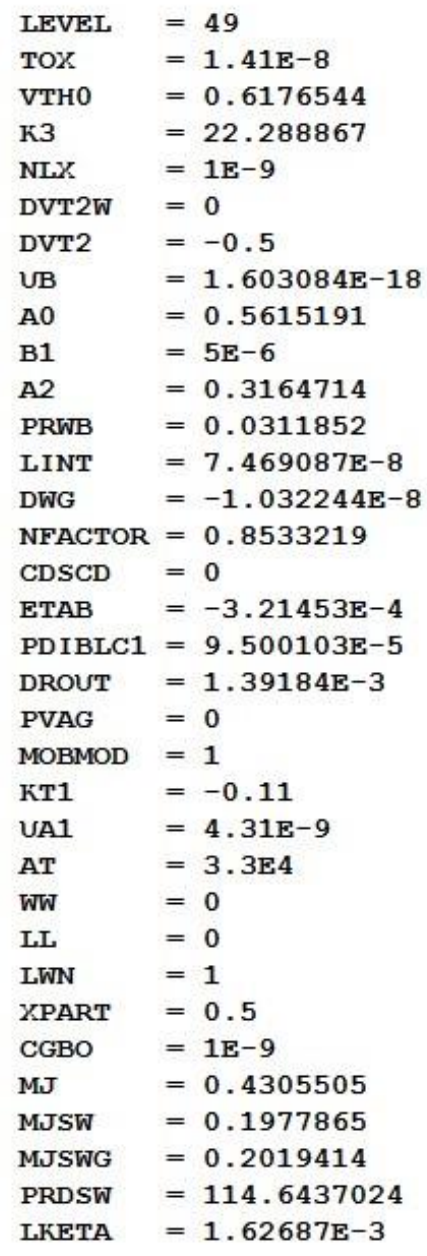




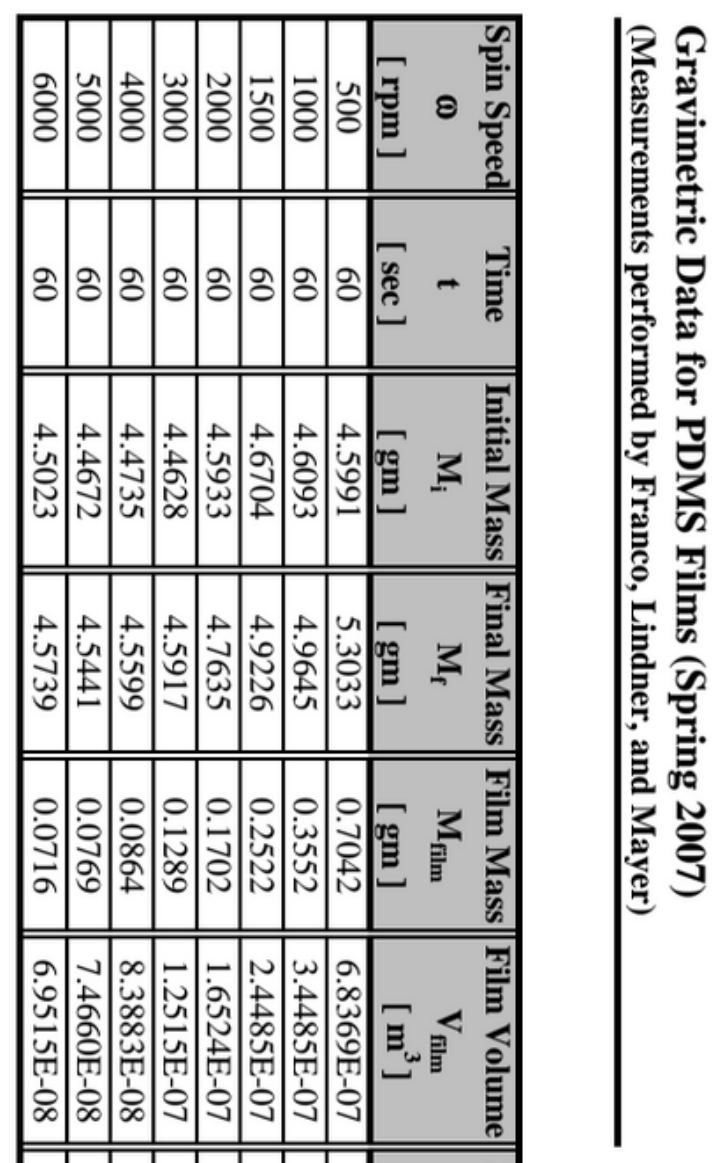




\section{Appendix B: Drawings}
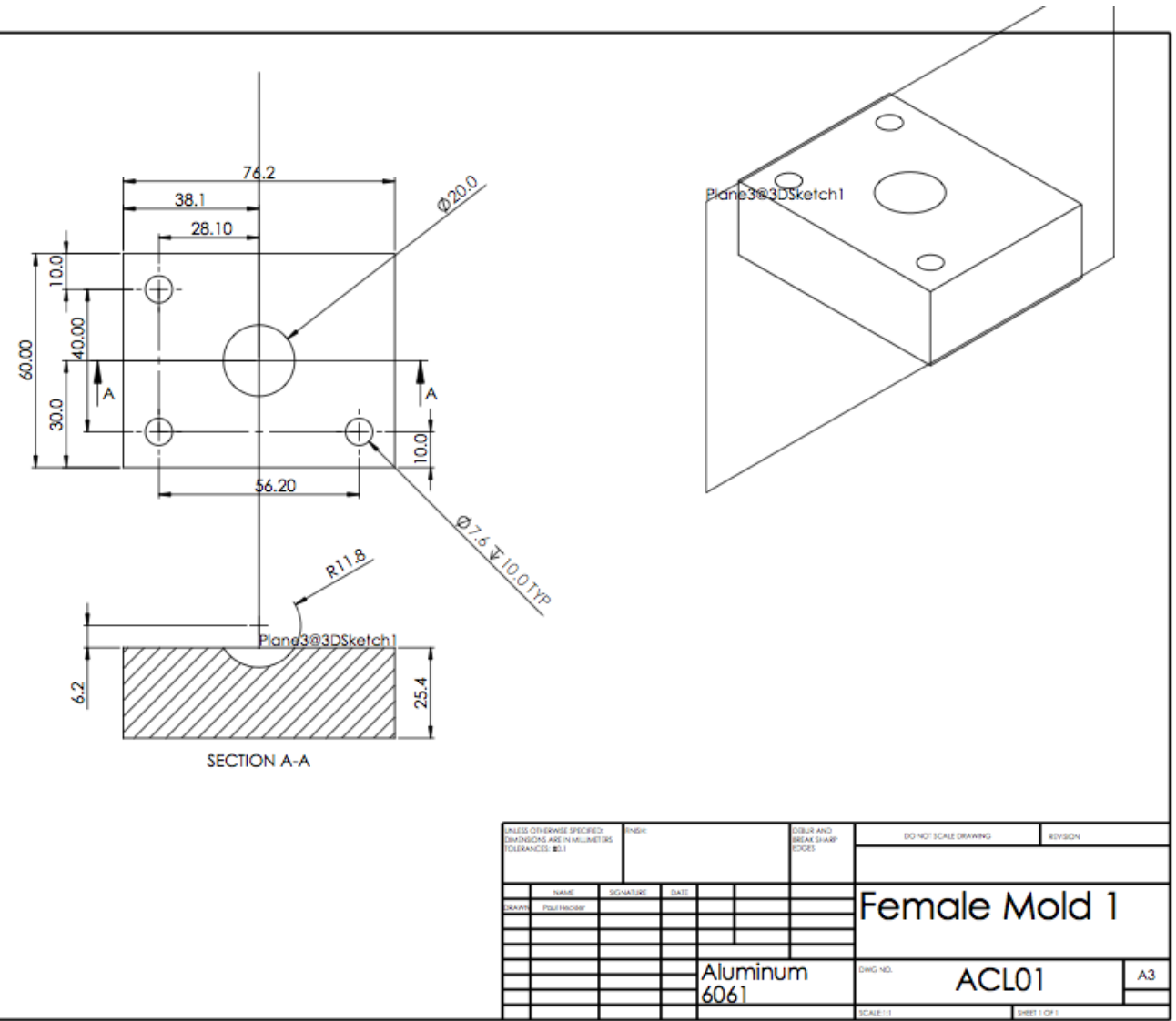

Figure 56: Drawing of Female Mold 1 


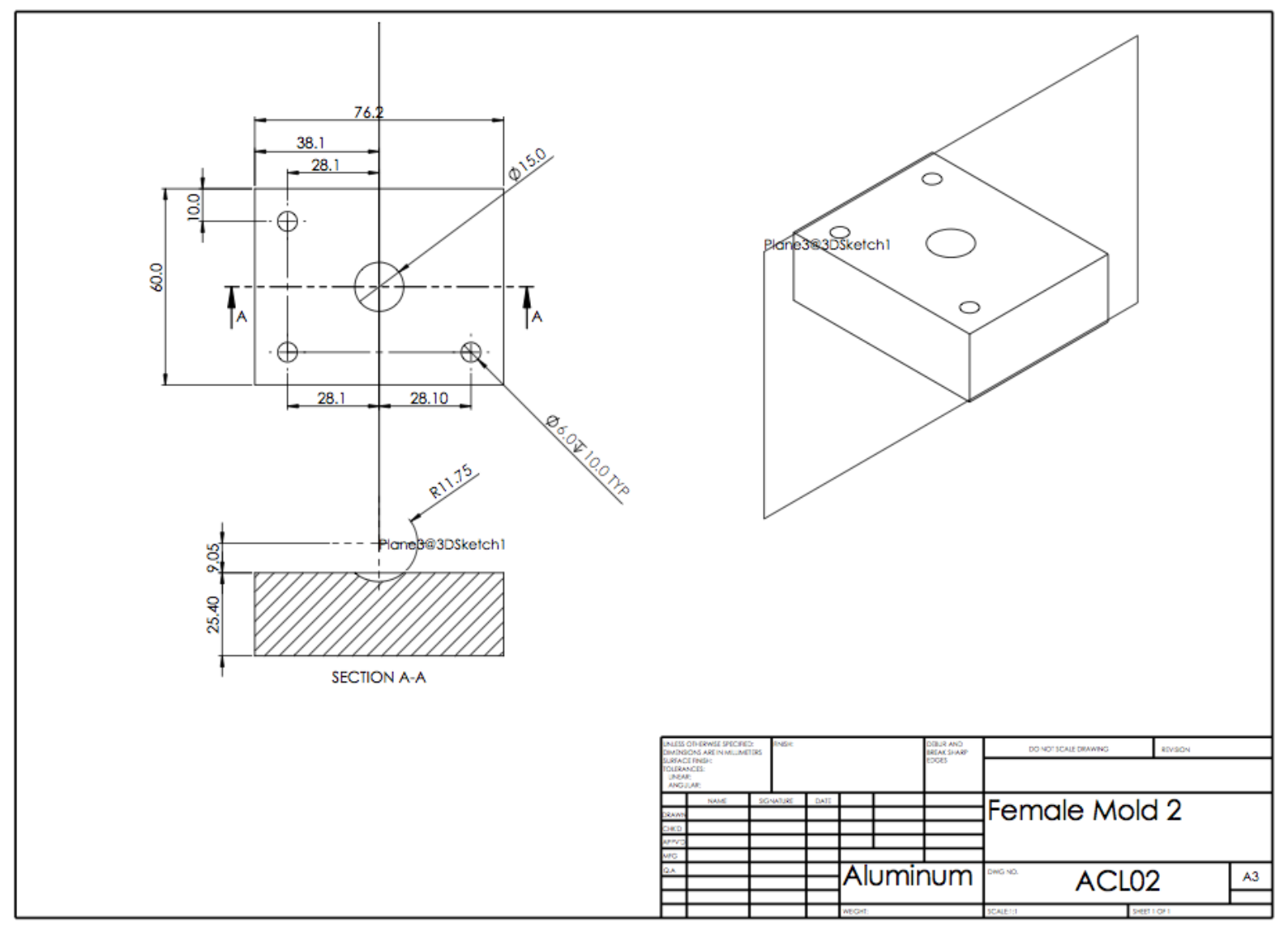

Figure 57: Drawing of Female Mold 2. 


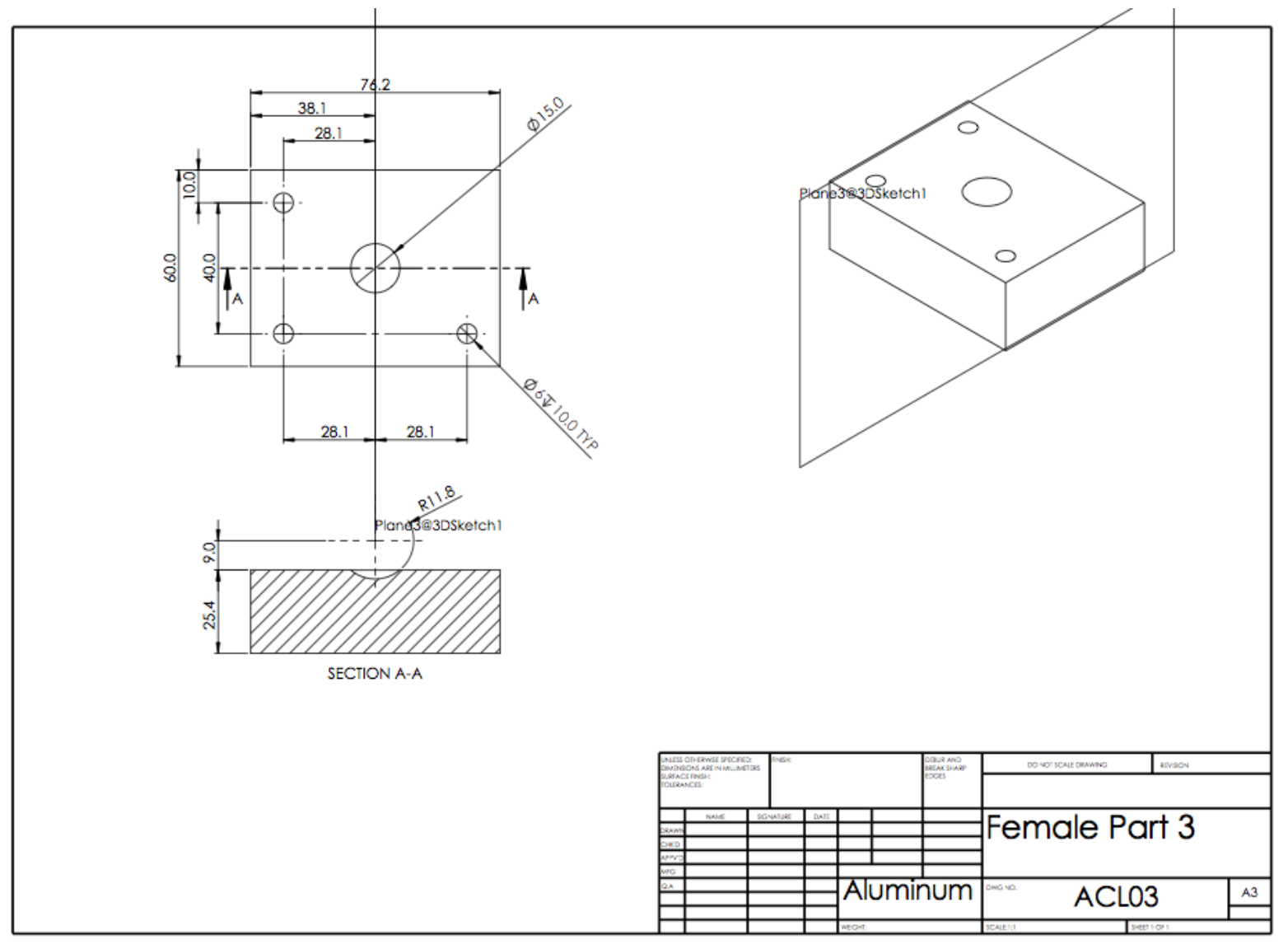

Figure 58: Drawing of Female Mold 3. 


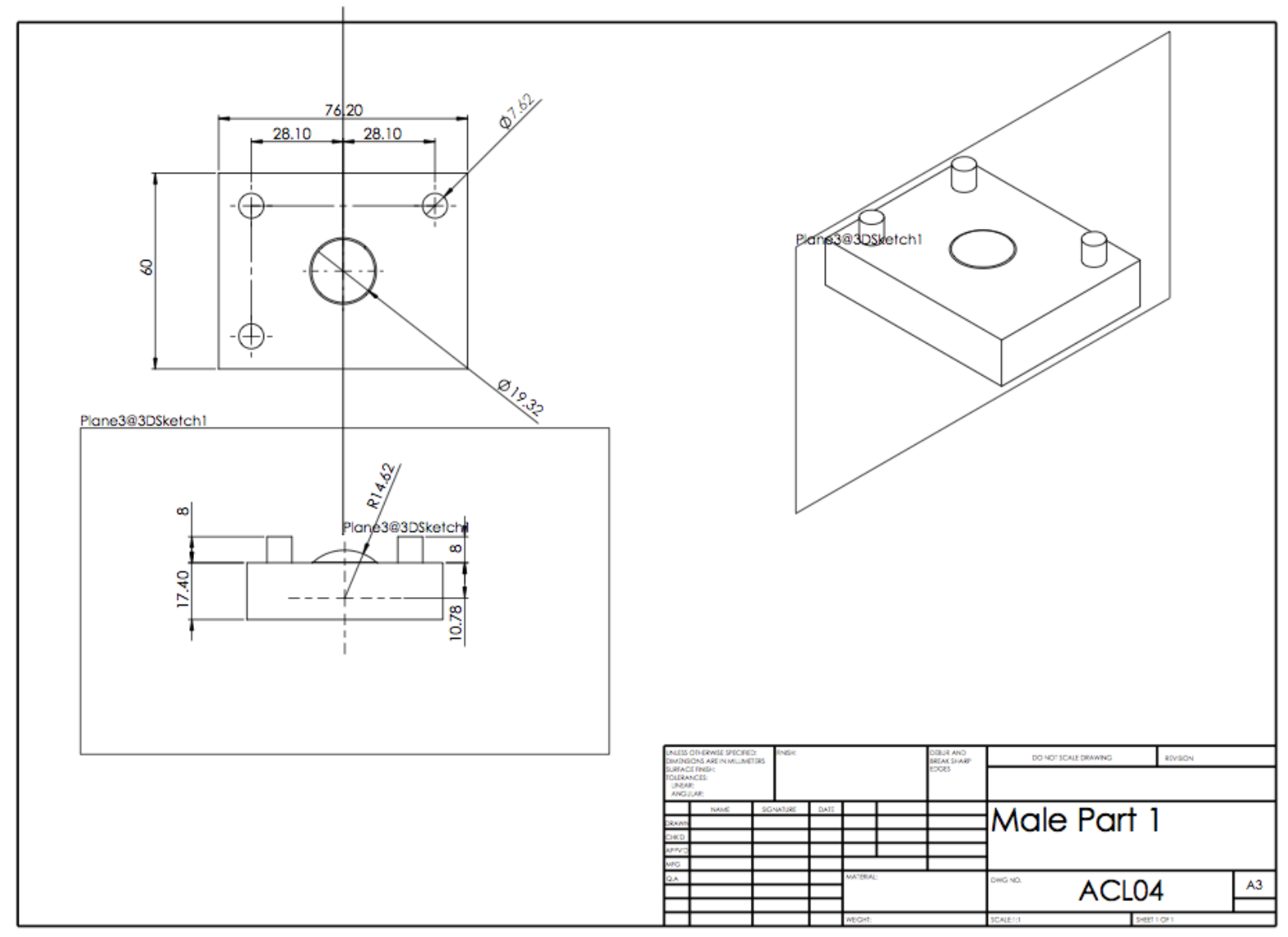

Figure 59: Drawing of Male Mold 1. 


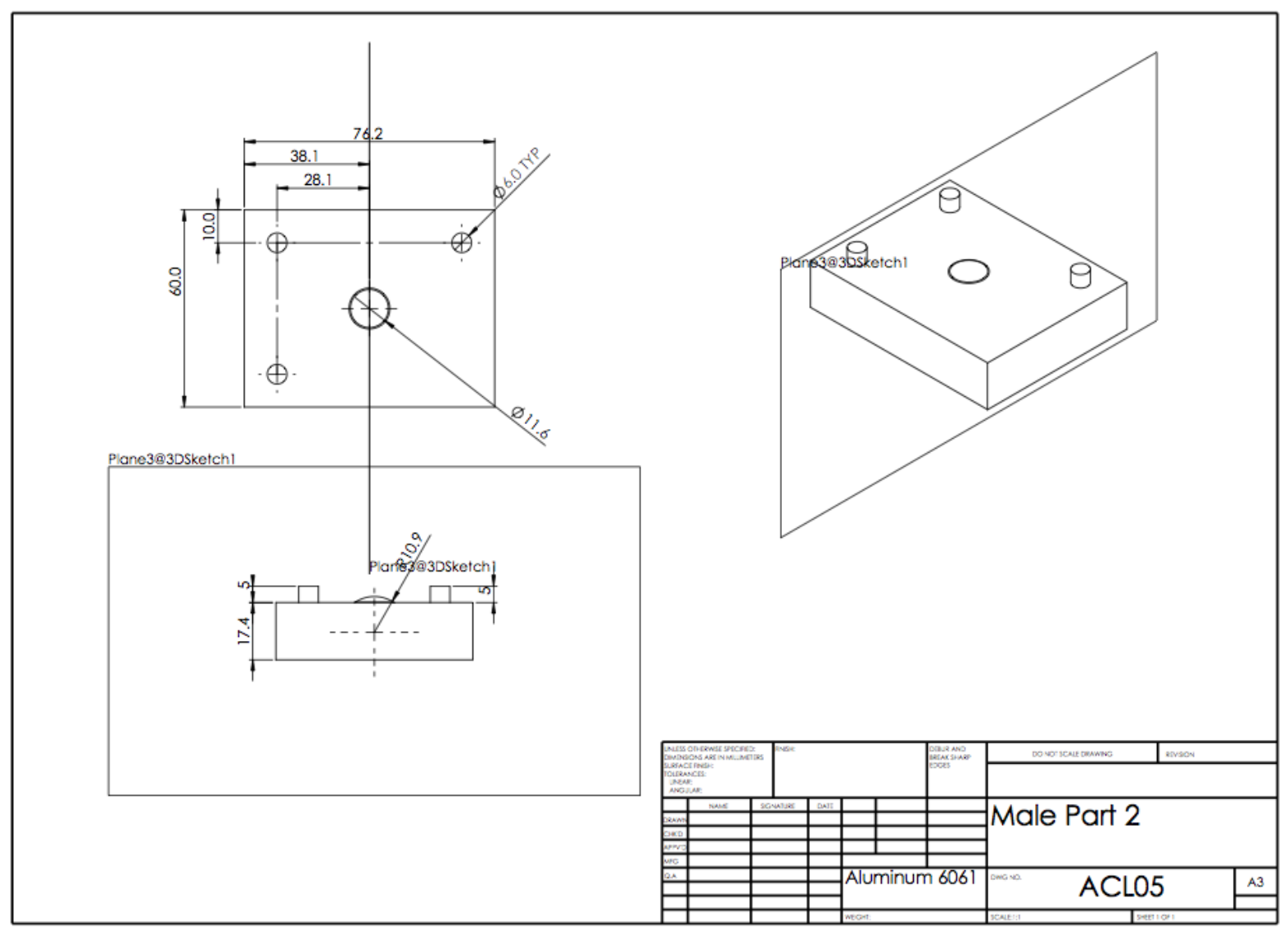

Figure 60: Drawing of Male Mold 2. 


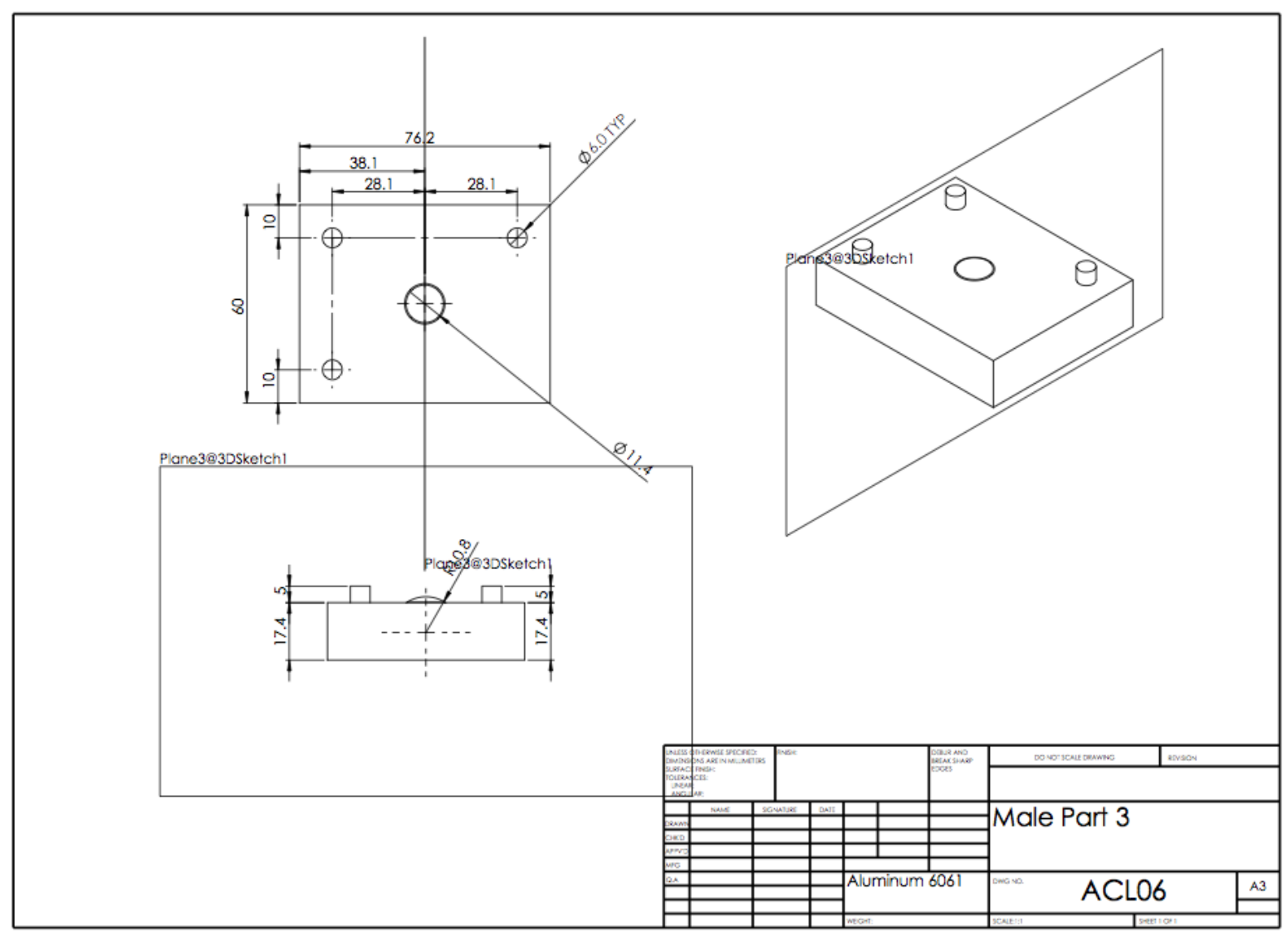

Figure 61: Drawing of Male Mold 3. 


\section{Appendix C: Cost of Materials}

- Cymbet CBC005 Thin Film battery: Qty $=143$, Total Cost $=\$ 250$ Cymbet $^{\mathrm{TM}}$ Corporation, 18326 Joplin Street NW, Elk River, MN, 55330-1773

Phone: 763.633 .1780

- Rohm Semiconductor Red LED: Qty $=10$, Total cost $=\$ 4.20$

- Rohm Semiconductor ML8511 UV Sensor: Qty $=5$, Total Cost $=\$ 38.30$ ROHM Semiconductor U.S.A.,LLC 6020 Cornerstone Court West, Suite 320, San Diego, CA 92121 U.S.A.

TEL : +1-858-625-3600 FAX : +1-858-625-3640

- Fairchild MMG3002MT1 RF amplifier: Qty $=3$, Total Cost $=\$ 14.94$

Fairchild Semiconductor Corporation 3030 Orchard Parkway

San Jose, CA 95134

U.S.A.

Tel: $408-822-2000$

- Maxim MAX2750EUA+2.4GHz oscillator: $\mathrm{Qty}=5$, Total Cost $=\$ 16.50$ Corporate Headquarters Maxim Integrated 160 Rio Robles San Jose, CA 95134 USA 408-601-1000

- One Industrial Way 2705 McMillan Ave, San Luis Obispo, CA 93401 (805) 596-0645

$1 \mathrm{x} 1 / 2 "$ carbide, 3 flute, flat end mill for aluminum (estimate $\sim \$ 30$ ea)

1x 1/4" carbide, 3 flute, flat end mill for aluminum (estimate $\sim \$ 25 e a$ )

2x 1/8" carbide, 3 flute, flat end mill for aluminum (estimate $~ \$ 20$ ea)

$2 \times 1 / 8 "$ carbide, 3 flute, ball end mill for aluminum (estimate $\sim \$ 15 e a$ )

$2 x 1 / 16 "$ carbide, 3 flute, ball end mill for aluminum (estimate $\sim \$ 15 e a$ )

- McMaster Carr 
9630 Norwalk Blvd.

Santa Fe Springs, CA 90670-2932

(562) 692-5911

$\begin{array}{llllrr}1.8975 \mathrm{~K} & \text { Multipur } & 2 & \text { to } & 24 & 4 \\ 239 & \text { pose } & \text { e } & \text { da } & .3 & 8 \\ & 6061 & \text { a } & \text { y } & 4 & . \\ & \text { Aluminu } & \text { c } & & \text { ea } & \\ & \text { m, 1" } & \text { h } & & \text { ch } & \\ & \text { Thick X } & & & & \\ & \text { 3" Width } & & & & \\ & \text { X 1' } & & & & \\ & \text { Length } & & & \end{array}$




\section{Appendix D: Gantt Chart for Research}

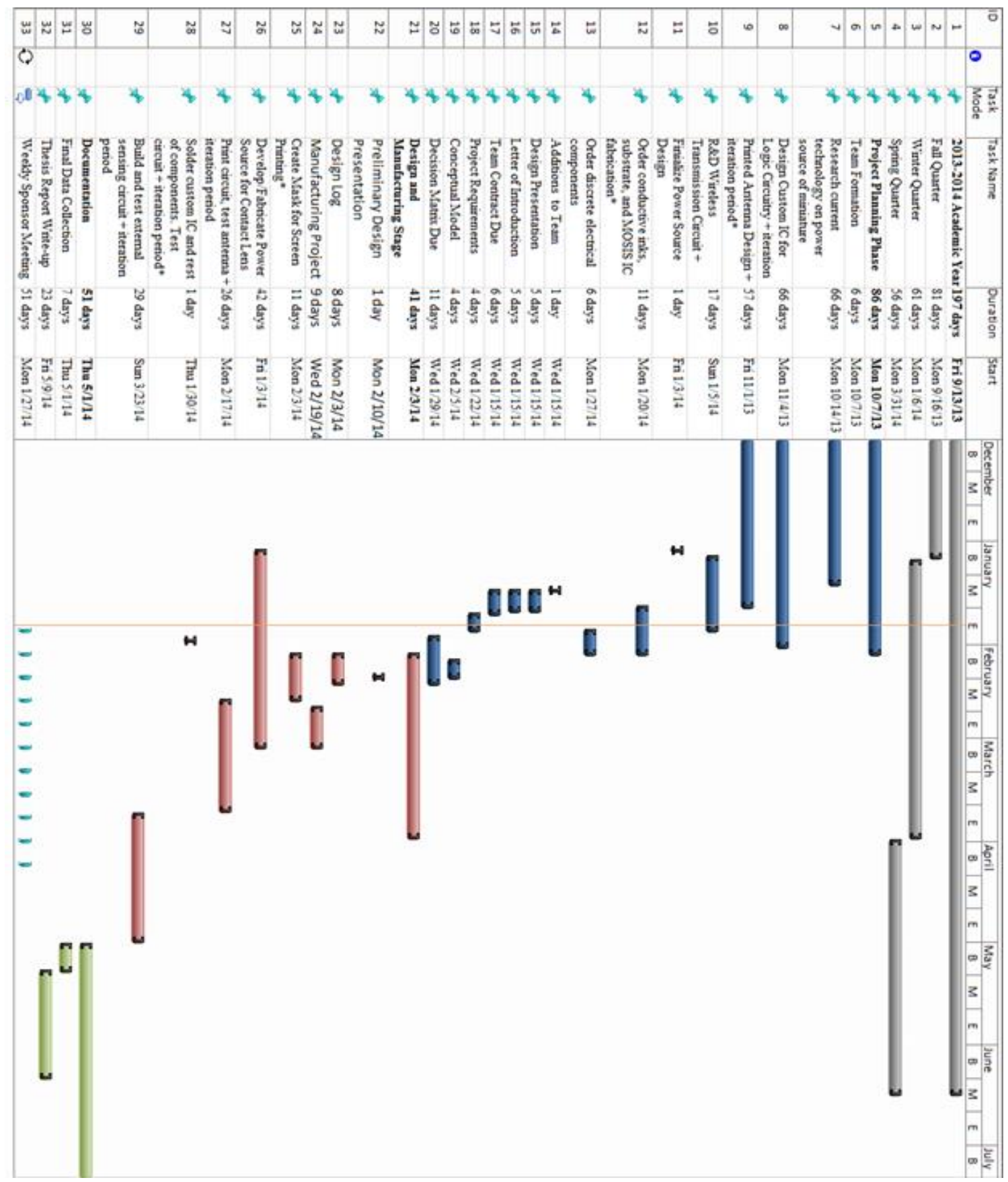

Figure 62: Gantt chart to reflect prior project status. 


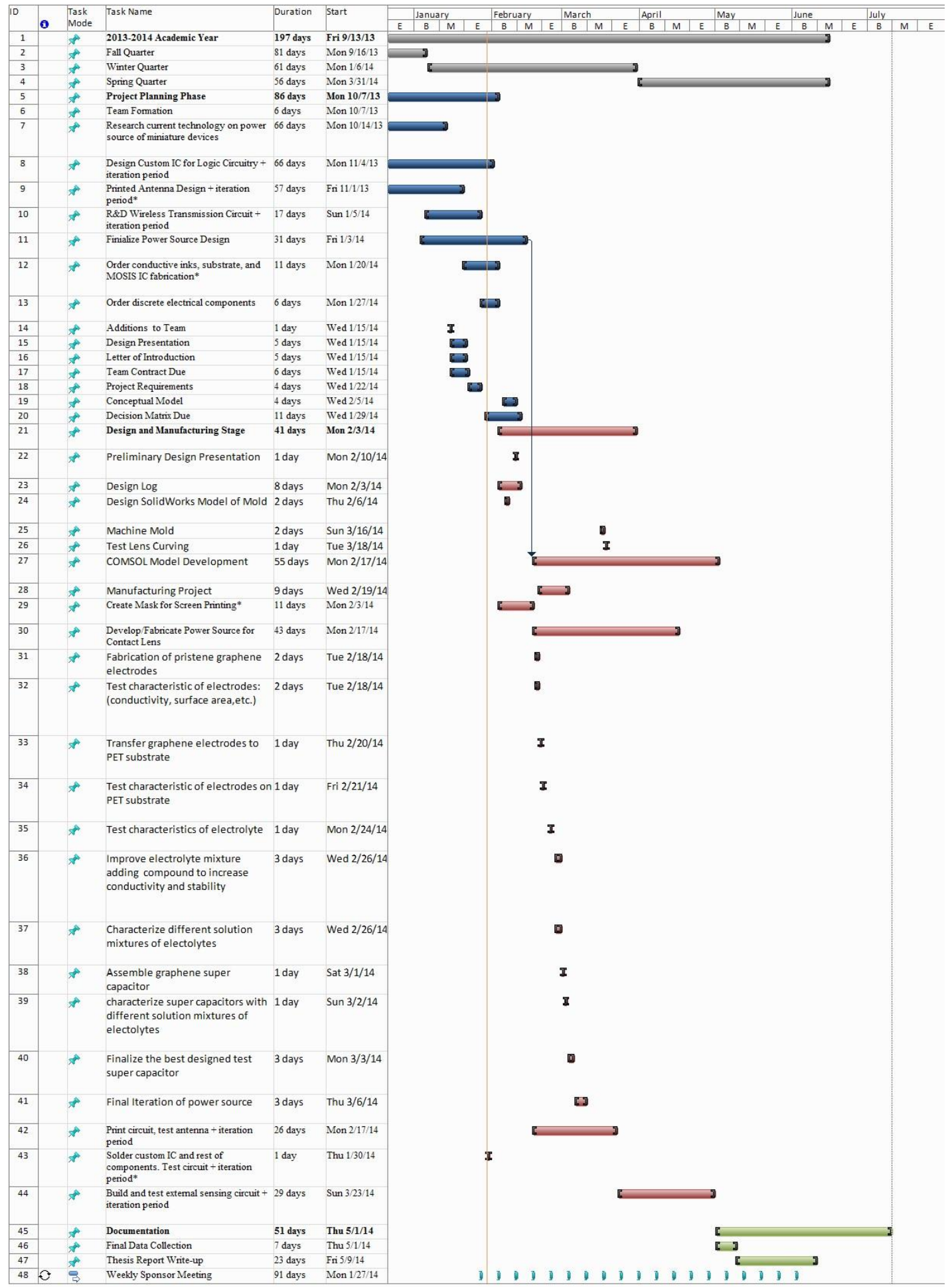

Figure 63: Updated Gantt chart to reflect most recent project status. 